\title{
From $Z Z$ to $Z H$ : How Low Can These Cross Sections Go or Everybody, Let's Cross Section Limbo!
}

\author{
A Dissertation Presented \\ by \\ Emanuel Alexandre Strauss \\ to \\ The Graduate School \\ in Partial Fulfillment of the Requirements \\ for the Degree of
}

Doctor of Philosophy

in

Physics

Stony Brook University

August 2009 


\section{Stony Brook University}

The Graduate School

\section{Emanuel Alexandre Strauss}

We, the dissertation committee for the above candidate for the Doctor of Philosophy degree, hereby recommend acceptance of this dissertation.

John D. Hobbs - Dissertation Advisor

Professor, Department of Physics and Astronomy

Paul D. Grannis - Chairperson of Defense

Professor, Department of Physics and Astronomy

George F. Sterman

Professor, Department of Physics and Astronomy

Thomas K. Hemmick

Professor, Department of Physics and Astronomy

Gregorio Bernardi

Dr, Directeur de Recherches au CNRS

Université Paris VII

This dissertation is accepted by the Graduate School.

Lawrence Martin

Dean of the Graduate School 
Abstract of the Dissertation

\title{
From $Z Z$ to $Z H$ : How Low Can These Cross Sections Go or Everybody, Let's Cross Section Limbo!
}

by

\author{
Emanuel Alexandre Strauss
}

Doctor of Philosophy

in

\section{Physics}

Stony Brook University

2009

\begin{abstract}
We report on two searches performed at the $D \varnothing$ detector at the Fermi National Laboratory. The first is a search for $Z$ di-boson production with a theoretical cross section of $1.4 \mathrm{pb}$. The search was performed on $2.6 \mathrm{fb}^{-1}$ of data and contributed to the first observation of $Z Z$ production at a hadron collider. The second is a search for a low mass Standard Model Higgs in $4.2 \mathrm{fb}^{-1}$ of data. The Higgs boson is produced in association with a $Z$ boson where the Higgs decays hadronically and the $Z$ decays to two leptons. The $Z Z$ search was performed in both the di-electron and di-muon channels. For the $Z H$ search, we will focus on the muonic decays where we expanded the traditional coverage by considering events in which one of the two muons fails the selection requirement, and is instead reconstructed as an isolated track. We consider Higgs masses between 100 and $150 \mathrm{GeV}$, with theoretical cross sections ranging from 0.17 to $0.042 \mathrm{pb}$, and set upper limits on the $Z H$ production cross-section at $95 \%$ confidence level.
\end{abstract}




\section{Contents}

1 Introduction $\quad 1$

1.1 The Particles of the Standard Model . . . . . . . . . . . . . . . . . . 2

1.1.1 Leptons and Quarks . . . . . . . . . . . . . . . . . . . . . . . . . . . . .

1.1 .2 The Force Mediators . . . . . . . . . . . . . . . . . . . 3

1.2 Formalism . . . . . . . . . . . . . . . . . . . . . 4

1.2.1 Quantum Chromodynamics .................. 4

1.2.2 The Electroweak Theory . . . . . . . . . . . . . . . . 5 5

1.2.3 Introducing the Higgs . . . . . . . . . . . . . . . . . . . . 6

1.2.4 The Gauge Interactions . . . . . . . . . . . . . . . . 8

1.3 Nomenclature . . . . . . . . . . . . . . . . . . . . . . . . 10

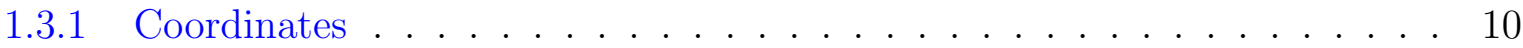

1.3 .2 Units . . . . . . . . . . . . . . . . . . . . 10

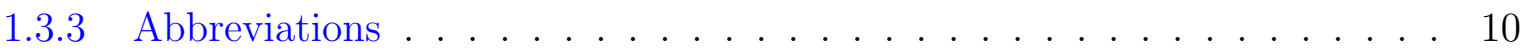

2 Experimental Apparatus 13

2.1 The Accelerators and The Tevatron . . . . . . . . . . . . . . . . . . . 13

2.1.1 Creating the Proton Beam . . . . . . . . . . . . . . . 13

2.1 .2 The Main Injector . . . . . . . . . . . . . . . . . . . . . . 16

2.1.3 Creating the Anti-Proton Beam . . . . . . . . . . . . . . . 16

2.1.4 The Tevatron . . . . . . . . . . . . . . . . 17

2.2 The $\mathrm{D} \varnothing$ Detector . . . . . . . . . . . . . . . . . . . 18

2.2 .1 Luminosity . . . . . . . . . . . . . . . . . . . . . 18

2.2.2 Tracker . . . . . . . . . . . . . . . . . . . . . . 19

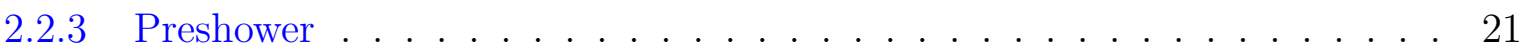

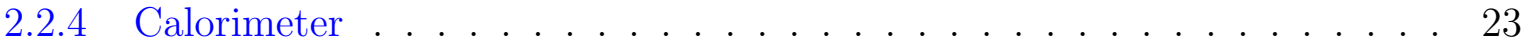

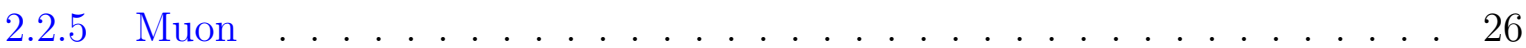

2.3 Triggering . . . . . . . . . . . . . . . . . . . . . . 27

2.3 .1 The Level 1 Trigger . . . . . . . . . . . . . . . . . . . 28

2.3.2 The Level 2 Trigger . . . . . . . . . . . . . . . . . . . . . . . . . 29

2.3.3 The Level 3 Trigger and Data Acquisition . . . . . . . . . . . . . . 30 
3 Monte Carlo Simulation $\quad 31$

3.1 Event Generators . . . . . . . . . . . . . . . . . . . 31

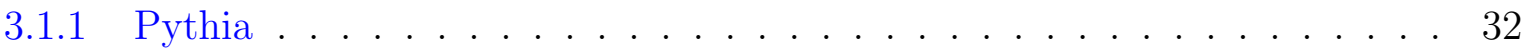

3.1 .2 Alpgen . . . . . . . . . . . . . . . . . . . . 32

3.1 .3 MLM Matching Scheme . . . . . . . . . . . . . . . . 32

3.2 Detector Simulation . . . . . . . . . . . . . . . . . . . . 33

3.2.1 Detector Material . . . . . . . . . . . . . . . . . 33

3.2 .2 Electronics . . . . . . . . . . . . . . . . . . . 33

3.3 Simulation Corrections . . . . . . . . . . . . . . . . . . . . 34

4 Reconstruction and Identification $\quad 36$

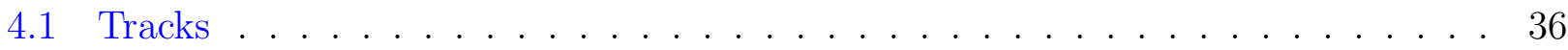

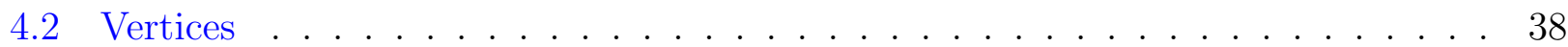

4.3 Muons . . . . . . . . . . . . . . . . . . . . . . 38

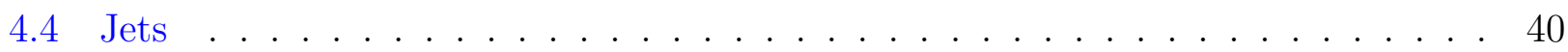

4.5 Electrons and Photons . . . . . . . . . . . . . . . . . . . . . . 42

4.6 Missing Energy . . . . . . . . . . . . . . . . . . . . . . . . . . 44

5 B-Tagging $\quad 46$

5.1 Taggability and Vertex Confirmed Jets . . . . . . . . . . . . . . . . 46

5.2 Secondary Vertex Tagger . . . . . . . . . . . . . . . . . . . . 47

5.3 The Jet LIfetime Probability Tagger . . . . . . . . . . . . . . . . . . . . . . . . 48

5.4 Counting Signed Impact Parameter Tagger . . . . . . . . . . . . . . . . . . . . . 49

5.5 The Neural Network Tagger . . . . . . . . . . . . . . . . . . . . . . . . . 50

$5.6 \quad b$ and $c$-jet Tagging Efficiency . . . . . . . . . . . . . . . . 51

5.7 Tag Rate Function . . . . . . . . . . . . . . . . . . . . . 52

6 Limit Setting $\quad 54$

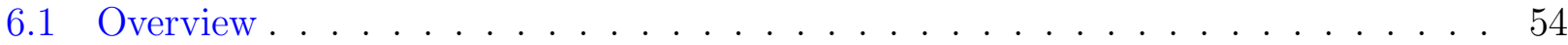

6.2 The Test Statistic . . . . . . . . . . . . . . . . . . . 55

6.2.1 The Log-Likelihood Ratio . . . . . . . . . . . . . . . . . . 55

6.2.2 Minimizing Systematics . . . . . . . . . . . . . . . 55

6.3 Confidence Levels and $p$-Values . . . . . . . . . . . . . . . . . . 56

6.4 Measuring the Cross Section . . . . . . . . . . . . . . . . . 57

$7 \quad Z Z \rightarrow \ell \ell \nu \nu$ Analysis $\quad 58$

7.1 Dataset and Monte Carlo Samples . . . . . . . . . . . . . . . . . . 60

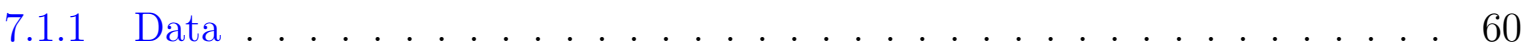

7.1 .2 Monte Carlo . . . . . . . . . . . . . . . . . . 60 60

7.1.3 Additional Monte Carlo Corrections . . . . . . . . . . . . . . . . . . . 60

7.2 Preselection Requirements . . . . . . . . . . . . . . . . . . 64 
7.2.1 Electron Requirements .................... 64

7.2 .2 Muon Requirements . . . . . . . . . . . . . . . . . . 64

7.2 .3 Jet Requirements . . . . . . . . . . . . . . . . . . . . . 65

7.2.4 Additional Leptonic Activity Requirements . . . . . . . . . . . . . . . . 65

7.3 Estimating Instrumental Backgrounds . . . . . . . . . . . . . . . . . . . . . . . . . . . . . . . . . . . . . 75

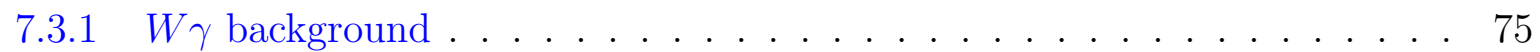

7.3.2 W+jets background . . . . . . . . . . . . . . 76

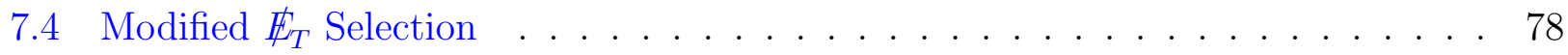

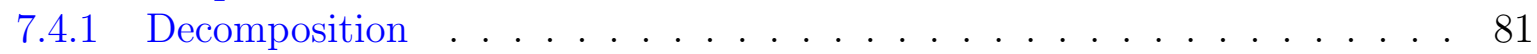

7.4 .2 Calorimeter Recoil . . . . . . . . . . . . . . . . . . . 83

7.4 .3 Recoiling Tracks . . . . . . . . . . . . . . . . . . . 86

7.4.4 Lepton $p_{T}$ uncertainty . . . . . . . . . . . . . . . . . . . . . 89

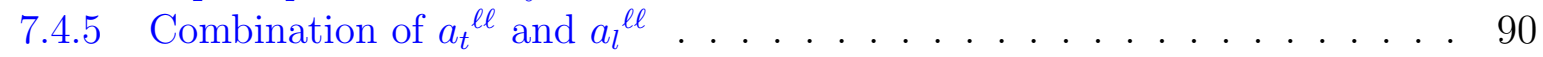

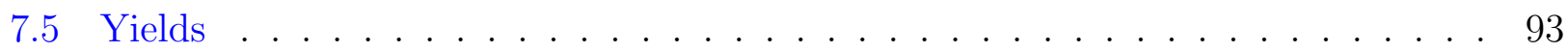

7.6 Likelihood Background Discriminant . . . . . . . . . . . . . . . . . . . . . 95

7.7 Systematic Uncertainties . . . . . . . . . . . . . . . . . . . . 102

7.7 .1 Significance Systematics . . . . . . . . . . . . . . . . . . . . . . . . . . . . . . . . . . . . .

7.7 .2 Cross Section Systematics . . . . . . . . . . . . . . . 103

7.8 Cross Section Measurement and Significance . . . . . . . . . . . . . . 109

$8 \quad Z H \rightarrow \ell \ell b b$ Analysis $\quad 113$

8.1 Dataset and Monte Carlo Samples . . . . . . . . . . . . . . . . . . 114

8.1 .1 Data . . . . . . . . . . . . . . . . . 114

8.1 .2 Monte Carlo . . . . . . . . . . . . . . . . . 115

8.1.3 Additional Monte Carlo Corrections . . . . . . . . . . . . . . . 115

8.2 Preselection Requirements . . . . . . . . . . . . . . . . . . . . . . . . 119

8.2.1 Muon Selection . . . . . . . . . . . . . . . . . . 119

8.2.2 Track-only Muon Selection . . . . . . . . . . . . . . . . 119

8.2 .3 Jet Selection . . . . . . . . . . . . . . . . . . . . 123

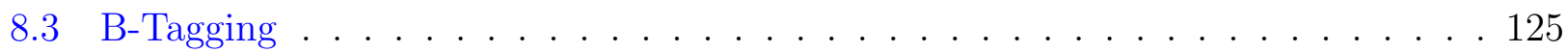

8.3.1 2L Inclusive Weighting . . . . . . . . . . . . . . . . 125

8.3.2 1T Exclusive Weighting . . . . . . . . . . . . . . . 125

8.4 Kinematic Fit . . . . . . . . . . . . . . . . . . . . 127

8.4 Implementation . . . . . . . . . . . . . . . . . . . . . . . . . . . . . . . . . . . . . . . . . . . . . . . .

8.4 Fit Performance . . . . . . . . . . . . . . . . . . 128

8.4.3 Cutting on the fit $\chi^{2} \ldots \ldots \ldots \ldots$. . . . . . . . . . . . . . . . . . . . . . . . . . . . . . . . . . . . . . . . . .

8.5 Yields . . . . . . . . . . . . . . . . . . . 136

8.6 Multivariate Discriminant . . . . . . . . . . . . . . . . . . . . . . . . . . . . . . . . . . . . . . . 137

8.6.1 Random Forest . . . . . . . . . . . . . . . . . . . . 137

8.6 .2 Technical Implementation . . . . . . . . . . . . . . . . . . . 138

8.6.3 Variable Selection . . . . . . . . . . . . . . . . . 138 
8.6.4 RF Performance . . . . . . . . . . . . . . . . . . . 139

8.7 Systematic Uncertainties . . . . . . . . . . . . . . . . . . . . . 143

8.8 Limits . . . . . . . . . . . . . . . . . . . . . . . 145

9 Conclusion and Outlook $r 4$

$\begin{array}{ll}\text { Bibliography } & 151\end{array}$

A Lepton Fake Rate $\quad 155$

A.1 Electron Isolation . . . . . . . . . . . . . . . . . . . . . . . . . . 155

A.2 Muon Isolation . . . . . . . . . . . . . . . . . . . 157

B Resolution Functions $\quad 161$

B.1 Muon $p_{T}$ Uncertainty . . . . . . . . . . . . . . . . . . . . . 161

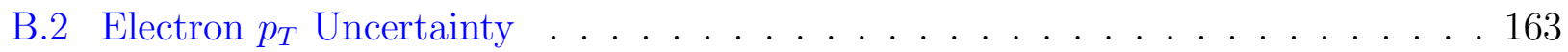

B.3 Jet $p_{T}$ Uncertainty . . . . . . . . . . . . . . . . . . . . . . . . 163

B.4 Lepton and Jet Angular Uncertainties . . . . . . . . . . . . . . . . . . 164

C Optimization of the $\mathbb{E}_{T}^{\prime} k$ and $k^{\prime}$ multipliers $\quad 166$

$\begin{array}{ll}\text { D Inclusive Trigger Modeling } & 168\end{array}$ 


\section{Chapter 1}

\section{Introduction}

"M'enfin! Ouvrez, quoi... J'ai du travail à faire, moi!"

Gaston Lagaffe

In particle physics, we concern ourselves with the development and understanding of the laws which govern the smallest and most elementary constituents of matter. These laws have been packaged into the Standard Model (SM), a Quantum Field Theory (QFT) which describes the fundamental particles and the forces that govern them. This introductory chapter paints a picture of the SM and motivates the searches we have performed for this thesis.

A significant amount of time and resources have gone into developing the tools we use in pursuit of our research goals. The studies described in this thesis were performed at DØ, a multi-purpose detector located at the Tevatron particle-accelerator at Fermilab. The running of the $\mathrm{D} \varnothing$ detector is a massive endeavour in and of itself, and the group of over 600 physicists who perform their research at $D \varnothing$ are as implicated in the upkeep and operation of the detector as they are in physics analyses. The long life of the Tevatron physics program is as much a testament to the hard work of the scientists and engineers who keep the aging apparatus operating ever more efficiently, as it is to the quality and interest of the science which it produces. Chapter 2 of this thesis will further describe the apparatus which makes our work possible.

The interpretation of our results requires a sophisticated modeling of both the physical processes that we are investigating and the performance of the detector used to aggregate the data. A method of Monte Carlo simulations is described in Chapter 3.

Priot to performing the analysis, the collected data is parsed as described in Chapter 4 . Physical objects are reconstructed by combining the information from several subdetector components to build an understanding of the particles produced in the initial collision of protons and anti-protons. 
As the focus of searches at $\mathrm{D} \emptyset$ has evolved over time, so too have the reconstruction algorithms. Of particular importance to searches involving $b$-quarks is our ability to determine the flavour of the progenitor quarks in our recorded events. These "tagging" tools have gained a significant level of sophistication and will be covered in Chapter 5.

The statistical methods of interpreting our data are covered in Chapter 6. Having properly collected (and analogously, modeled) the data, a rigorous method of assessing the sensitivity of our results is described there.

All of this leads us to the two analyses: a search for $Z Z$ di-boson production in Chapter 7 and a search for a Higgs boson produced in association with a $Z$ boson in Chapter 8. Both of these are very rare physics processes. We will focus on demonstrating the techniques which were developed to increase our sensitivity to the signal through novel methods of rejecting the background, increasing our signal acceptance, and by improving the resolution of analysis variables.

\subsection{The Particles of the Standard Model}

We will treat our introduction to the SM in two passes. In this section, we provide a brief overview of the relevant concepts. A more technical description is reserved for Section 1.2.

\subsubsection{Leptons and Quarks}

Ordinary matter, of the type we interact with every day, is made up of infinitesimally small particles called fermions. These particles are referred to as fundamental because, as far as we know, they are indivisible and have no further sub-structure. The fermions all have half-integer angular momentum, or spin, in units of $\hbar$ and obey Fermi-Dirac statistics. Due to the Pauli Exclusion Principle, they must have unique quantum states, such that no two fermions may occupy the same state if they have the same spin, charge, etc ....

There are two fermionic building blocks: Leptons and Quarks. Nature has ordered fermions into three distinct generations containing a quark with positive charge of $+\frac{2}{3}$, a quark with negative charge of $-\frac{1}{3}$, a lepton with charge -1 , and a neutral lepton (neutrino) with exceedingly small mass which barely interacts with most matter. Each of these has an associated anti-particle with opposite quantum numbers. The known fermions, as well as some of their properties, are shown in Table 1.1.

Whereas leptons, such as electrons, may exist freely in nature, quarks cannot due to the principle of confinement (See Section 1.1.2). Instead, they must group together in twos (mesons) or threes (baryons). The two most commonly known groupings of quarks are the proton and the neutron. These composite particles, referred to as hadrons, have a signature mass and charge derived from their constituent quarks. In the case of a proton, two $u$ quarks with charge

$+\frac{2}{3}$ and one $d$ quark with charge $-\frac{1}{3}$, give it a charge of +1 . The neutron is made up of two $d$ quarks and one $u$ quark, giving it a net charge of 0 . Because of the large amount of energy 
Table 1.1: The three generations of fermions (anti-particles not shown here). The three families have identical gauge interactions (See Section 1.2), but differ by their mass and flavour quantum number.

\begin{tabular}{|l|cc|cc|cc|}
\hline \hline \multirow{2}{*}{ Type } & \multicolumn{2}{|c|}{$1^{\text {st }}$ Generation } & \multicolumn{2}{c|}{$2^{\text {nd }}$ Generation } & \multicolumn{2}{c|}{$3^{\text {rd }}$ Generation } \\
& Particle & Mass $(\mathrm{MeV})$ & Particle & Mass $(\mathrm{MeV})$ & Particle & Mass $(\mathrm{MeV})$ \\
\hline Charged Lepton & Electron $(e)$ & 0.511 & Muon $(\mu)$ & 105.7 & Tau $(\tau)$ & 1776.8 \\
Neutrino & $\nu_{e}$ & $<2 \times 10^{-6}$ & $\nu_{\mu}$ & $<0.19$ & $\nu_{\tau}$ & $<18.2$ \\
+ Charged Quark & up $(u)$ & 1.5 to 3.3 & charm $(c)$ & 1.27 & top $(t)$ & 171.2 \\
- Charged Quark & down $(d)$ & 3.5 to 6.0 & strange $(s)$ & 104 & bottom $(b)$ & 4.20 \\
\hline \hline
\end{tabular}

associated with the strong force, the mass of hadrons is not given directly by the sum of the quarks.

\subsubsection{The Force Mediators}

The forces between fundamental particles of the SM are mediated by particles with integer spin called Bosons. The exception to this is gravity, which is not described by the SM. Bosons obey Bose-Einstein statistics, meaning that several bosons with identical energy and quantum numbers can occupy the same state. The Electromagnetic, Weak, and Strong forces are described in the following sub-sections, and a summary of the force mediators is provided in Table 1.2.

Table 1.2: The force mediators of the SM, along with their mass and spin. Also included is the Higgs boson which is covered in further detail in Section 1.2.3.

\begin{tabular}{|l|ccc|}
\hline \hline Particle & Mass $(\mathrm{GeV})$ & Spin & Force \\
\hline photon $(\gamma)$ & 0 & 1 & Electromagnetic \\
$Z^{0}$ & 91.1876 & 1 & Weak \\
$W^{ \pm}$ & 80.398 & 1 & Weak \\
gluon & 0 & 1 & Strong \\
\hline Higgs $(H)$ & $114.5<m_{H}<160$ or $m_{H}>170$ & 0 & - \\
\hline \hline
\end{tabular}

\section{Electromagnetic Force}

Charged particles are the domain of the electromagnetic (EM) force, which is experienced, for example, every time lightning strikes, or when two magnets are brought into close proximity of each other. Photons, which couple to all particles with electric charge, are its force mediators, and since photons are massless the range of the EM force is infinite. 


\section{Weak Force}

The weak force is responsible for radioactive $\beta$ decay, in which a neutron decays to a proton, electron, and anti-neutrino $\left(n \rightarrow p+e^{-}+\bar{\nu}_{e}\right)$. There are two types of weak processes:

- Charged weak processes are mediated by the two charged bosons, the $W^{+}$and $W^{-}$.

- Neutral weak processes are mediated by the neutral $Z^{0}$ boson.

Since all three of these bosons have significant mass, the strength of this force decreases rapidly with distance.

\section{Strong Force}

The strong force is responsible for holding the nucleus of atoms together. There are 8 mediating massless gauge bosons called gluons which couple to quarks, but not to leptons. The formalism which describes the strong force interactions introduces "color" quantum numbers which come in red, green, and blue. As with the EM force, the mediators are massless. Because the strength of the strong force grows with increasing distance quarks cannot exist alone, an effect which is referred to as confinement.

\subsection{Formalism}

As briefly mentioned above, the SM is a theory of relativistic quantum fields, in which quantum mechanics and relativity have been combined. In QFT, particles are manifestations of a field that takes on distinct values for any position in space and time. The values are calculated using a Lagrangian; a mathematical construction which describes a physical system and its evolution. A number of local continous symmetries are observed in nature, and the Lagrangian can be constructed to obey them by being made gauge invariant. This means that the Lagrangian is invariant under a group of transformations and that the field is invariant under the transformation $\psi(x) \rightarrow e^{i \alpha(x)} \psi(x)$. It is local because the change in phase of the wave function is allowed to be different at every point in space and time. All of this comes at the expense of the numbers of degrees of freedom of the system. For each of these symmetry groups, there is a corresponding gauge field, which, when quantized, gives rise to the force-mediating bosons. The Standard Model's symmetry group $(U(1) \times S U(2) \times S U(3))$ gives rise to 12 bosons: The photon, the $W^{+}$, the $W^{-}$, the $Z^{0}$, and eight gluons.

\subsubsection{Quantum Chromodynamics}

Quantum Chromodynamics (QCD), describes the strong interaction which controls the dynamics of the quarks (See 1.1.2). QCD must satisfy the color symmetry observed in nature, 
as well as the observations that there are three colors for the quarks and anti-quarks. $S U(3)$ satisfies each of these conditions and the QCD Lagrangian is given by Equation 1.1 [1].

$$
\mathcal{L}_{Q C D} \equiv-\frac{1}{4} G_{a}^{\mu \nu} G_{\mu \nu}^{a}+\sum_{f} \bar{q}_{f}^{\alpha}\left(i \gamma^{\mu} D_{\mu}-m_{f}\right) q_{f}^{\alpha}
$$

In this equation, $q_{f}^{\alpha}$ is a quark field of colour $\alpha$ and flavour $f$. The gluon field strength tensor $\left(G_{\mu \nu}^{i}\right)$ is defined as [2]:

$$
G_{\mu \nu}^{i}=\partial_{\mu} G_{\nu}^{i}-\partial_{\nu} G_{\mu}^{i}-g_{s} f_{i j k} G_{\mu}^{j} G_{\nu}^{k}
$$

and the covariant derivative $D_{\mu}$ is given by:

$$
D_{\mu}=\partial^{\mu}+i g_{s} G^{\mu}
$$

$G^{\mu}$ is a $3 \times 3$ matrix formed by the eight color gauge fields corresponding to the eight gluons.

Note that the QCD gauge coupling constant $\left(g_{s}\right)$ appears in Equation 1.2, setting the strength of the gluon self-interactions produced by the expansion of the $G_{a}^{\mu \nu} G_{\mu \nu}^{a}$ terms.

Expanding and re-arranging Equation 1.1 we get the following:

$$
\begin{array}{r}
\mathcal{L}_{Q C D}=-\frac{1}{4}\left(\partial^{\mu} G_{a}^{\nu}-\partial^{\nu} G_{a}^{\mu}\right)\left(\partial_{\mu} G_{\nu}^{a}-\partial_{\nu} G_{\mu}^{a}\right) \\
+\sum_{f} \bar{q}_{f}^{\alpha}\left(i \gamma^{\mu} \partial_{\mu}-m_{f}\right) q_{f}^{\alpha} \\
-g_{s} G_{a}^{\mu} \sum_{f} \bar{q}_{f}^{\alpha} \gamma_{\mu}\left(\frac{\lambda^{a}}{2}\right)_{\alpha \beta} q_{f}^{\beta} \\
+\frac{g_{s}}{2} f^{a b c}\left(\partial^{\mu} G_{a}^{\nu}-\partial^{\nu} G_{a}^{\mu}\right) G_{\mu}^{b} G_{\nu}^{c}-\frac{g_{s}^{2}}{4} f^{a b c} f_{a d e} G_{b}^{\mu} G_{c}^{\nu} G_{\mu}^{d} G_{\nu}^{e}
\end{array}
$$

where the first line contains the kinetic terms, the second line gives the colour interactions between quarks and gluons, and the third gives the cubic and quartic gluon self-couplings. Gluons do not couple exclusively to quarks, since they have color, but are able to couple to themselves as well.

\subsubsection{The Electroweak Theory}

As with the formulation of QCD for strong interactions, there are a number of constraints which help us build a formalism for Electromagnetic and Weak interactions. It has been observed that only left-handed (right-handed) fermions (anti-fermions) participate in flavour-changing weak interactions and that leptons appear in a doublet containing a charged and neutral component. Neutrinos on the other hand are known to be left-handed.

The $S U(2) \otimes U(1)$ symmetry group, which is the basis of the Electroweak theory, satisfies these conditions. Its Lagrangian is shown in Equation 1.5 for a single generation of leptons. This simplification ignores the need for a matching quark doublet for the leptons, as well as 
the two other generations, but the generalization will be enough to serve our purpouses.

$$
\begin{array}{r}
\mathcal{L}_{E W K}=\underbrace{-\frac{1}{4} W_{\mu \nu}^{i} W^{\mu \nu i}-\frac{1}{4} B_{\mu \nu} B^{\mu \nu}}_{\mathcal{L}_{\text {gauge }}} \\
+\underbrace{i \bar{R} \gamma^{\mu}\left(\partial_{\mu}+i g^{\prime} \frac{Y}{2} B_{\mu}\right) R+i \bar{L} \gamma^{\mu}\left(\partial_{\mu}+i g^{\prime} \frac{Y}{2} B_{\mu}+i g \tau^{i} W_{\mu}\right) L}_{\mathcal{L}_{\text {lep }}^{1}}
\end{array}
$$

The $\mathcal{L}_{\text {gauge }}$ terms are as follows:

$$
\begin{array}{cl}
W_{\mu \nu}^{j}=\partial_{\mu} W_{\nu}^{j}-\partial_{\nu} W_{\mu}^{j}+g \epsilon^{j k l} W_{\mu}^{k} W_{\nu}^{l} & \text { The } S U(2) \text { gauge field with } j=1,2,3 \\
B_{\mu \nu}=\partial_{\mu} B_{\nu}-\partial_{\nu} B_{\mu} & \text { The } U(1) \text { gauge field }
\end{array}
$$

The coupling constant of the $S U(2)(U(1))$ group is denoted $g\left(g^{\prime}\right)$. The $L$ term is the left-handed isospin doublet of the fermion $-\left(\begin{array}{c}\nu_{i} \\ \ell_{i}^{-}\end{array}\right)_{L}$. Since there is no sign of interactions with right-handed neutrinos, $R$, the right-handed component, only a singlet $-\left(\ell_{i}\right)_{R}$. We have only shown the Lagrangian for one flavour, $i=1$, so $\nu_{i}=\nu_{e}$ and $\ell_{i}=e$.

The $W^{ \pm}, \gamma$, and $Z^{0}$ bosons do not appear explicitly here, but the Lagrangian terms may be re-written such that:

$$
\begin{aligned}
W_{\mu}^{ \pm}=\frac{1}{\sqrt{2}}\left(W_{\mu}^{1} \mp W_{\mu}^{2}\right) & \text { The } W^{ \pm} \text {gauge boson } \\
Z_{\mu}=-\sin \theta_{W} B_{\mu}+\cos \theta_{W} W_{\mu}^{3} & \text { The } Z^{0} \text { gauge boson } \\
A_{\mu}=\cos \theta_{W} B_{\mu}+\sin \theta_{W} W_{\mu}^{3} & \text { The } \gamma \text { photon } \\
\cos \theta_{W}=\frac{g}{\sqrt{g^{2}+g^{\prime 2}}} & \text { The Weinberg angle }
\end{aligned}
$$

$\cos \theta_{W}$ is the Weinberg angle, which determines the mixing of the $W_{\mu}^{i}$ and $B_{\mu}$ terms.

\subsubsection{Introducing the Higgs}

As formulated in Section 1.2.2, the gauge symmetry of the electroweak theory does not allow mass terms for the leptons and bosons (for example, of the form $\frac{1}{2} m^{2} W_{\mu \nu}^{i} W^{\mu \nu i}$ ), as this would not respect the gauge invariance. The photon is massless, but since the $W^{ \pm}$and $Z^{0}$ of the weak interaction are known to be massive, the theory predicts three more massless bosons than we expect in the SM. The processes of Spontaneous Symmetry Breaking, where the vacuum state of a symmetric system does not preserve the symmetry, is used to resolve this problem.

To give these particles the mass they so rightly deserve, we must add new terms to the 
electroweak Lagrangian. These terms must preserve the symmetry of $\mathcal{L}_{E W K}$ while generating masses for the $W^{ \pm}$and $Z^{0}$, but not the photon.

The need to maintain the Lorentz invariance means that the additional term must be a scalar field, so we introduce a complex doublet of scalar fields with weak hypercharge $Y_{\phi}=+\frac{1}{2}$, as given in Equation 1.12, by adding Equation 1.13 to the Lagrangian.

$$
\begin{gathered}
\Phi \equiv\left(\begin{array}{c}
\phi^{+} \\
\phi^{0}
\end{array}\right) \\
\mathcal{L}_{\phi}=\left(\mathcal{D}^{\mu} \Phi\right)^{\dagger}\left(\mathcal{D}_{\mu} \Phi\right)-V(\Phi)
\end{gathered}
$$

where the gauge-covariant derivative is:

$$
\mathcal{D}_{\mu}=\partial_{\mu}+i g \frac{\tau^{i}}{2} W_{\mu}^{i}+\frac{i g^{\prime}}{2} B_{\mu}
$$

and $\tau^{i}$ are the Pauli matrices.

The second term in Equation 1.13, $V(\Phi)$, is the Higgs potential and has the form:

$$
V(\Phi)=\mu^{2} \Phi^{\dagger} \Phi+\lambda\left(\Phi^{\dagger} \Phi\right)^{2}
$$

We can further constrain the terms, since we know that the vacuum expectation value of the charged component of $\Phi$ must be zero to preserve the $U(1)$ symmetry of the vacuum. The potential must be bounded from below, so $\lambda>0$. For the $\mu$ term however there are two options:

- $\mu^{2}>0$ : The minimum is at $\langle 0|\Phi| 0\rangle \geq 0$ and no spontaneous symmetry breaking occurs. We are left with the same initial problem of having too many massless Bosons in the SM.

- $\mu^{2}<0$ : The minimum is obtained by:

$$
\langle 0|\Phi| 0\rangle=\left(\begin{array}{c}
0 \\
\phi_{0}
\end{array}\right) \quad \phi_{0}=\sqrt{\frac{-\mu^{2}}{2 \lambda}} \equiv \frac{v}{\sqrt{2}}>0 \quad V\left(\phi_{0}\right)=\frac{\lambda}{4} v^{4}
$$

$v$, the vacuum expectation value can be derived from the coupling constant of weak interactions and is known to be $246 \mathrm{GeV}$.

In this scenario, there are an infinite number of degenerate minimum energy states where $\phi_{0}(x)=\frac{v}{\sqrt{2}} e^{i \theta}$ since the Lagrangian is phase invariant. The breaking of $S U(2) \otimes U(1)$ occurs once we pick a particular value for $\theta$ - the simplest being $\theta=0$. In so doing, the lowest energy state no longer respects the gauge symmetry and it induces effective masses for the particles propagating through the field. Whereas before we had a complex scalar field $(\Phi)$ and a massless vector field, we now have a single scalar field corresponding to a spinless boson of mass $m_{H}=\sqrt{-2 \mu^{2}}=\sqrt{2 \lambda v^{2}}$ 
Direct searches for the Higgs Boson are performed with the hope of constraining the mass of the Higgs boson, and therefore of $\mu$ as well.

We are also free to add terms introducing the interactions between the scalar fields and the leptons. The Yukawa Lagrangian (See Equation 1.17) demonstrates this, again with only one lepton generation.

$$
\mathcal{L}_{\text {Yukawa }}=\Gamma_{i}\left[\bar{R}\left(\left(i \sigma_{2} \phi^{*}\right)^{\dagger} L\right)+\left(\bar{L}\left(i \sigma_{2} \phi^{*}\right)\right) R\right]
$$

Where $R$ and $L$ have been previously defined in Section 1.2.2 and $\Gamma_{i}$ are the matrices which describe the Yukawa couplings between the single Higgs doublet, $\Phi$, and the quarks and leptons. Since we have once again restricted ourselves to one lepton generation, $i=1$ and $\Gamma_{i}=\Gamma_{e} . \Phi$ and its conjugate can then be used to generate all of the fermion masses.

\subsubsection{The Gauge Interactions}

Since the second analysis in this thesis is a search for $Z Z$ di-boson production, it behooves us to take a moment and examine the couplings of the bosons in a little more depth. First we will cover the interactions between the bosons of the weak force and the fermions.

The weak force described in 1.1.2 involves either charged or neutral processes mediated by the $W^{ \pm}$and $Z^{0}$ bosons, respectively. The Lagrangian involving these two types of interactions can be split into a charged current term (See Equation 1.18) that couples the $W^{ \pm}$to charged left-handed fermions, and a neutral current term (See Equation 1.19) that couples the $Z^{0}$ to to neutrinos or charged leptons which are either right or left-handed.

$$
\mathcal{L}_{C C}=-\frac{g}{2 \sqrt{2}}\left(J_{W}^{\mu} W_{\mu}^{-}+J_{W}^{\mu \dagger} W_{\mu}^{+}\right)
$$

Where $J_{W}^{\mu}$ is the weak charged current.

$$
\mathcal{L}_{N C}=-\frac{\sqrt{g^{2}+g^{\prime 2}}}{2} J_{Z}^{\mu}\left(-\sin \theta_{W} B_{\mu}+\cos \theta_{W} W_{\mu}^{3}\right)
$$

Where $J_{Z}^{\mu}$ is the weak neutral current.

The previous two equations describe the interactions between the force-mediating bosons of the weak force and the fermions, giving rise to processes such as $Z \rightarrow \ell^{+} \ell^{-}$.

For the $Z Z$ analysis we are interested in the self-couplings of these bosons. When expanded, the kinetic terms in the $\mathcal{L}_{\text {gauge }}$ lagrangian (See Equation 1.5) lead to 3 and 4-point gauge self-interactions in the $W$ 's described by Equation 1.20 and Equation 1.21.

$$
\begin{aligned}
\mathcal{L}_{3}= & -i g\left(\partial_{\rho} W_{\nu}^{3}\right) W_{\mu}^{+} W_{\sigma}^{-}\left[g^{\rho \mu} g^{\nu \sigma}-g^{\rho \sigma} g^{\nu \mu}\right] \\
& -i g\left(\partial_{\rho} W_{\mu}^{+}\right) W_{\nu}^{3} W_{\sigma}^{-}\left[g^{\rho \sigma} g^{\mu \nu}-g^{\rho \nu} g^{\mu \sigma}\right] \\
& -i g\left(\partial_{\rho} W_{\mu}^{-}\right) W_{\nu}^{3} W_{\mu}^{+}\left[g^{\rho \nu} g^{\mu \sigma}-g^{\rho \mu} g^{\nu \sigma}\right]
\end{aligned}
$$




$$
\mathcal{L}_{4}=\frac{g^{2}}{4}\left[W_{\mu}^{+} W_{\nu}^{+} W_{\sigma}^{-} W_{\rho}^{-} \mathcal{Q}^{\mu \nu \rho \sigma}-2 W_{\mu}^{+} W_{\nu}^{3} W_{\sigma}^{3} W_{\rho}^{-} \mathcal{Q}^{\mu \nu \rho \sigma}\right.
$$

where

$$
\mathcal{Q}^{\mu \nu \rho \sigma}=2 g_{\mu \nu} g_{\rho \sigma}-g_{\mu \rho} g_{\nu \sigma}-g_{\mu \sigma} g_{\nu \rho}
$$

As before, the $Z$, and $\gamma$ bosons are supper-positions of the $W^{3}$ and $B$ terms (See Equation 1.9 and Equation 1.10). Notice that every term in $\mathcal{L}_{3}$ and $\mathcal{L}_{4}$ has a contribution from one of the charged $W$ fields, whereas none involve only a $Z$ or $\gamma$. This means that the SM does not include any local $Z Z Z, Z Z \gamma$, or $Z \gamma \gamma$ vertices. The agreement of the $W W$ and $Z Z$ cross sections therefore provide a direct experimental test of the gauge self-interactions. 


\section{$1.3 \quad$ Nomenclature}

\subsubsection{Coordinates}

The D $\varnothing$ experiment uses a right-handed spherical co-ordinate system. $z$ points along the direction of the proton beam, $\phi$ is the azimuthal angle in the plane perpendicular to the detector, and $\theta$ is the polar angle.

The pseudorapidity $(\eta)$ is usually used instead of the polar angle. It is defined as:

$$
\eta=-\ln \left(\tan \left(\frac{\theta}{2}\right)\right)
$$

For the type of boosted particles studied at D $\varnothing$, where the mass is negligable compared to the energy, $\eta$ approximates the rapidity $(y)$.

$$
y=\frac{1}{2} \ln \left(\frac{E+p_{z}}{E-p_{z}}\right)
$$

\subsubsection{Units}

We use a unit system commonly refered to as "Planck units", where a set of physical constants, such as $c$ the speed of light and $\hbar$ the planck constant, have been normalized to 1 to simplify certain equations. As a result we express mass in units of $\mathrm{GeV}$, rather than $\mathrm{GeV} / c^{2}$.

The rate of production for physical processes, their cross section, is defined in units of barns. One barn is equal to $10^{-24} \mathrm{~cm}^{2}$. Typically we refer to these in units of pico-barns, $10^{-15}$ barns.

Luminosity, which is a measure of the rate of inelastic $p \bar{p}$ collisions, is measured in units of inverse barns per second $\left(\mathrm{pb}^{-1} \mathrm{~s}^{-1}\right)$. The size of our accumulated dataset is measured as integrated luminosity over time, in units of $\mathrm{pb}^{-1}$. Luminosity will be discussed in more detail in Section 2.2.1.

\subsubsection{Abbreviations}

The following abbreviations are commonly used throughout this thesis. Although defined in the text before their first use, they are also collected here as an easily accessible reference.

- $p_{T}$ : The transverse momentum of a particle or system

- $E_{T}$ : The transverse energy of a particle or system

- $\mathbb{E}_{T}$ : The negative $E_{T}$, typically of an event, but in some cases of a subset of objects.

- $q$ : The charge of a particle or of an object

- CC: The Central Calorimeter 
- EC: The Endcap Calorimeter

- EM: Electromagnetic. This is often used as shorthand for the electromagnetic layers of the calorimeter or for electromagnetic particles such as photons and electrons.

- HAD: Hadronic. This is typically used as shorthand for the hadronic layers of the calorimeter.

- FH: Fine Hadronic layers of the calorimeter

- CH: Coarse Hadronic layers of the calorimter

- TOT: Total. This is typically used as shorthand for the combination of the EM and HAD calorimeter layers.

- PV: Primary Vertex

- CFT: Central Fiber Tracker

- SMT: Silicon Microstrip Tracker

- NDF: Number of Degree of Freedom

- dof: Degrees of Freedom

- BDT: Boosted Decision Tree. A pattern recognition technique used to classify signal-like and background-like events.

- RF: Random Forest. A pattern recognition technique used to classify signal-like and background-like events.

- MVA: Multivariate Discriminant.

- CL: Confidence Level

- JES: The Jet Energy Scale

- JER: Jet Energy Resolution

- MC: Monte Carlo

- ISR: Initial State Radiation

- FSR: Final State Radiation

- QCD: Quantum Chromo-Dynamics 
- QED: Quantum Electro-Dynamics

- PDF: Parton Distribution Function or Probability Density Function

- LO: Leading Order. Used in reference to the calculation of cross sections.

- NLO: Next To Leading Order. Used in reference to the calculation of cross sections.

- NNLO: Next to Next To Leading Order. Used in reference to the calculation of cross sections.

- LL: Leading Logarithmic. Used in reference to the calculation of cross sections.

- Fermilab: Fermi National Accelerator Laboratory

- SM: Standard Model

- NN: Neural Network

- LLR: Log-Likelihood Ratio

- DCA: Distance of Closest Approach

- IP: Impact Parameter

- $p \bar{p}$ : Proton and Antiproton 


\title{
Chapter 2
}

\section{Experimental Apparatus}

\author{
"Regardez: J'introduis dans l'appareil \\ un Ver Luisant. C'est lui qui, en \\ montant et descendant le long du'une \\ petite échelle, allumera successivement \\ les trois couleurs... Lààà! Sois brillant, \\ mon gars ..."
}

The Tevatron is a proton-antiproton $(p \bar{p})$ collider run by the Fermi National Accelerator Laboratory (Fermilab). Operating at a center-of-mass energy of $\sqrt{s}=1.96 \mathrm{TeV}$, it is currently the world's highest energy collider. The data described in this thesis were collected from April 2002 through September 2008 at the DØ detector, one of two detectors located at the Tevatron.

In this chapter we will describe the hardware which makes such an experiment possible. An aerial view and sketch of the Tevatron is provided in Figure 2.1 [3].

\subsection{The Accelerators and The Tevatron}

\subsubsection{Creating the Proton Beam}

With one proton and one electron, hydrogen is the closest, readily available, substance we have to protons. Hydrogen ions $\left(\mathrm{H}^{-}\right)$are produced by injecting hydrogen gas into a magnetron chamber [4] like the one sketched in Figure 2.2. In this chamber a cathode is fixed in the center while the walls act as the anode. The electric field strips away the electrons from the hydrogen molecules, creating a plasma. The positively charged ions strike the surface of the cathode, picking up extra electrons and becoming negatively charged. This system replaces a higher current positive Hydrogen ion $\left(\mathrm{H}^{+}\right)$source which produced a beam of inferior quality in the 


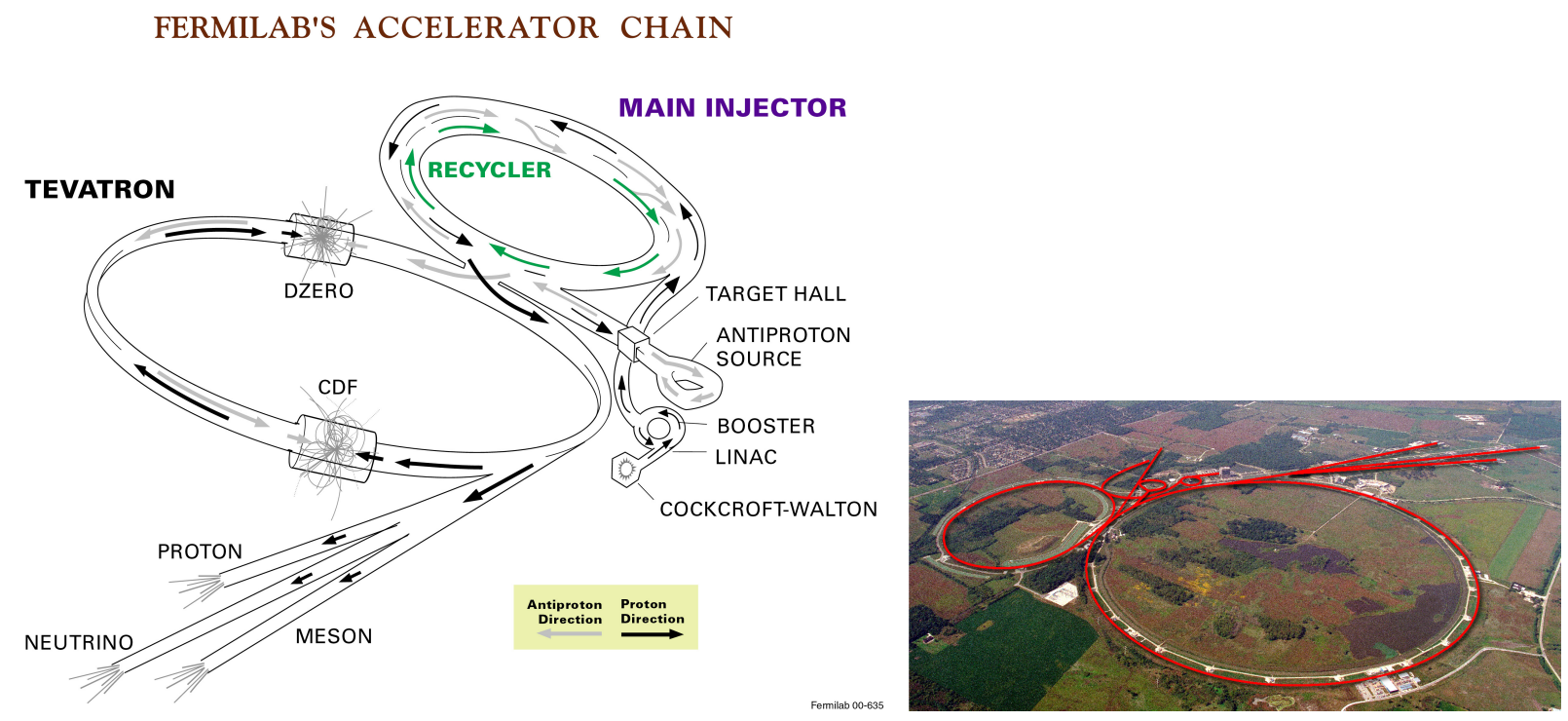

Figure 2.1: Schematic and aerial view of the Tevatron complex [3].

Booster system, described later in this chapter. An $18 \mathrm{kV}$ electric field guides the $\mathrm{H}^{-}$ions towards a Cockcroft-Walton generator.

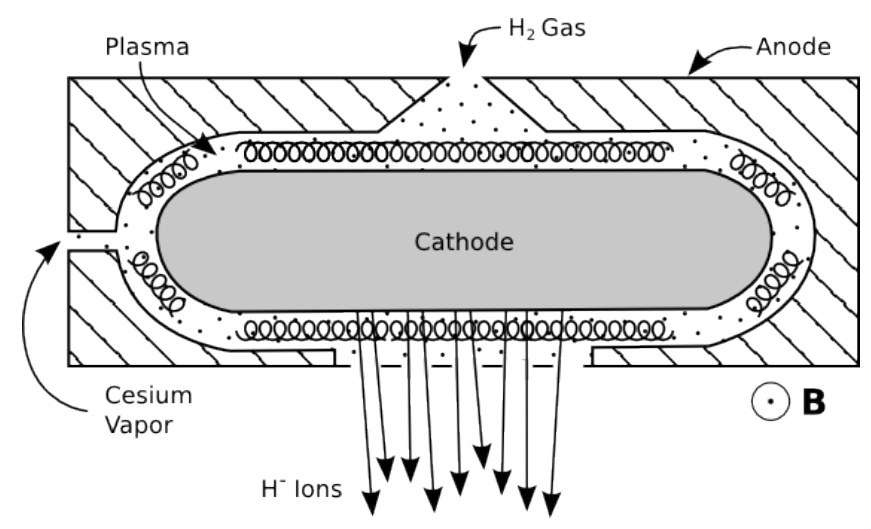

Figure 2.2: $\mathrm{H}_{2}$ molecules injected into the magnetron chamber exit as negatively charged $\mathrm{H}^{-}$ions.

The Cockcroft-Walton generator works in much the same way as a cathode tube. Capacitors are charged in parallel from an $\mathrm{AC}$ source and are then discharged in series - producing a continuous field of $750 \mathrm{kV} . \mathrm{H}^{-}$ions pick up energy as they travel along this field, down the length of the Cockcroft-Walton generator shown in Figure 2.3.

Having been accelerated to $750 \mathrm{kV}$, the $\mathrm{H}^{-}$ions are bunched by a single-gap radio frequency $(\mathrm{RF})$ cavity and fed into the Linear Accelerator (Linac). The Linac design is based on the use 


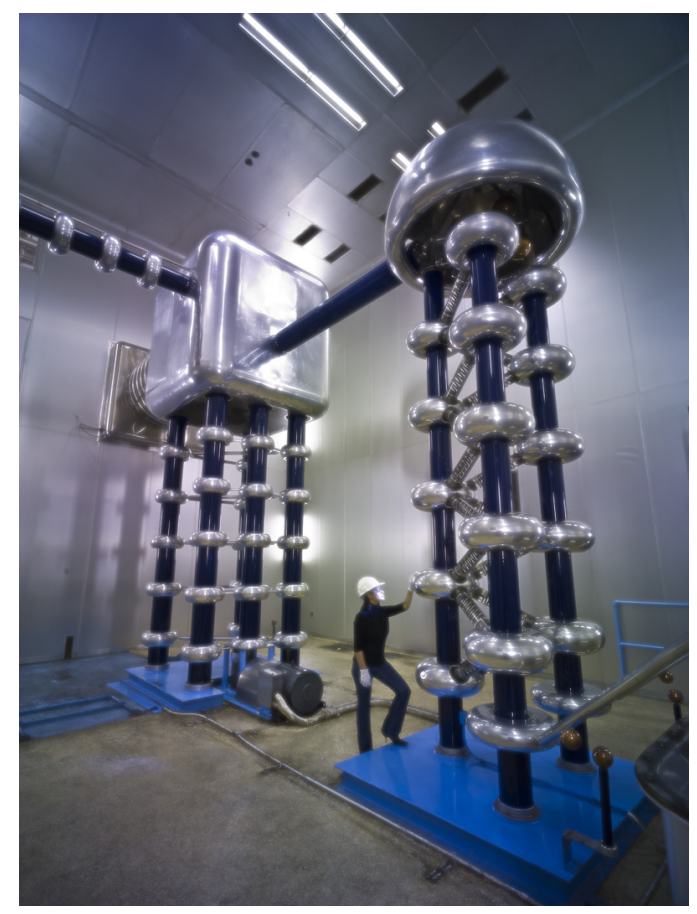

Figure 2.3: Photo of the Cockcroft-Walton generator.

of RF cavities - a series of drift regions separated by gaps. Charged particles within the gaps are accelerated by the flux from the varying magnetic field, those in the drift region are shielded from it. The length of the cells is such that particles are in the gap between the drift region when the acceleration direction is positive, and are in the drift region and shielded from the field when the acceleration direction is negative. The nominal in-gap time of the $\mathrm{H}^{-}$is shorter than one RF cycle such that particles which are slightly ahead of the bunch will gain less energy, whereas those slightly behind the bunch will gain more. This 500 foot long device boosts the $\mathrm{H}^{-}$ions to energies of $400 \mathrm{MeV}$ while at the same time transforming the beam from a continuous stream of particles into pulses with a frequency of $201.25 \mathrm{MHz}$.

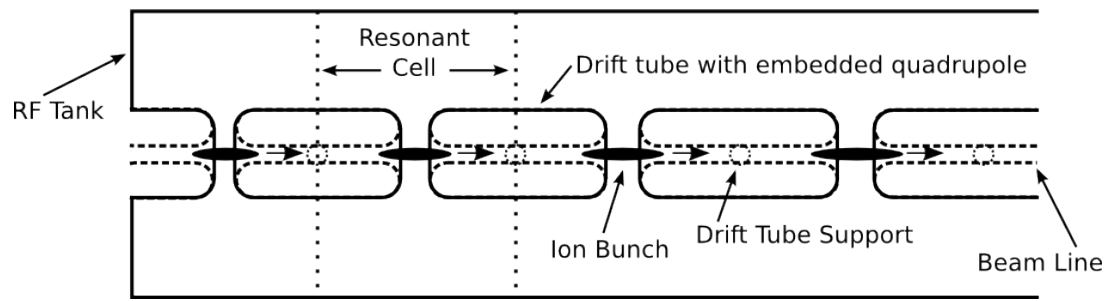

Figure 2.4: Particle bunches are accelerated by the Linac, traveling along the RF cavities.

Size constraints make it impractical to accelerate these particles to energies above a few 
hundred $\mathrm{MeV}$ using a Linac. In the next stage, the $\mathrm{H}^{-}$bunches travel through a carbon foil, stripping the electrons and leaving only the protons. They then enter the Booster - a $475 \mathrm{~m}$ circumference synchrotron. The Booster is supplied with approximately $3 \times 10^{12}$ protons by the Linac before accelerating them to $8 \mathrm{GeV}$ by varying the RF frequency from 37.9 to 52.813 $\mathrm{MHz}$ over a period of $33 \mathrm{~ms}$.

\subsubsection{The Main Injector}

The proton beam from the previous chapter is further accelerated by the Main Injector. This too is a synchrotron, but with a circumference of $3.3 \mathrm{~km}$. It accelerates both protons (produced as described in the previous section) and anti-protons (produced as described in the following section) to energies of $150 \mathrm{GeV}$ at which point it injects them into the Tevatron. The Main Injector also boosts protons to energies of $120 \mathrm{GeV}$ to be delivered to the anti-proton source.

\subsubsection{Creating the Anti-Proton Beam}

$120 \mathrm{GeV}$ protons $(p)$ are shot at a fixed nickel target in the aptly named Target Station. The collisions produce a shower of particles consisting of muons, pions, kaons, and most importantly anti-protons. The production cross section for $p+\mathrm{Ni} \rightarrow \bar{p}+X$ is roughly constant for $\bar{p}$ energies between 7.2 and $7.8 \mathrm{GeV}$, so the resulting anti-protons have an energy spread of $\approx 4 \%$. The extraction of these anti-protons is costly, only 1 being produced for each $10^{5}$ incoming protons.

A $15 \mathrm{~cm}$ long lithium lens, with a radius of $1 \mathrm{~cm}$, is used to focus the resultant broadly distributed beam. The beam is then transported through a magnetic field to separate the positively and negatively charged particles.

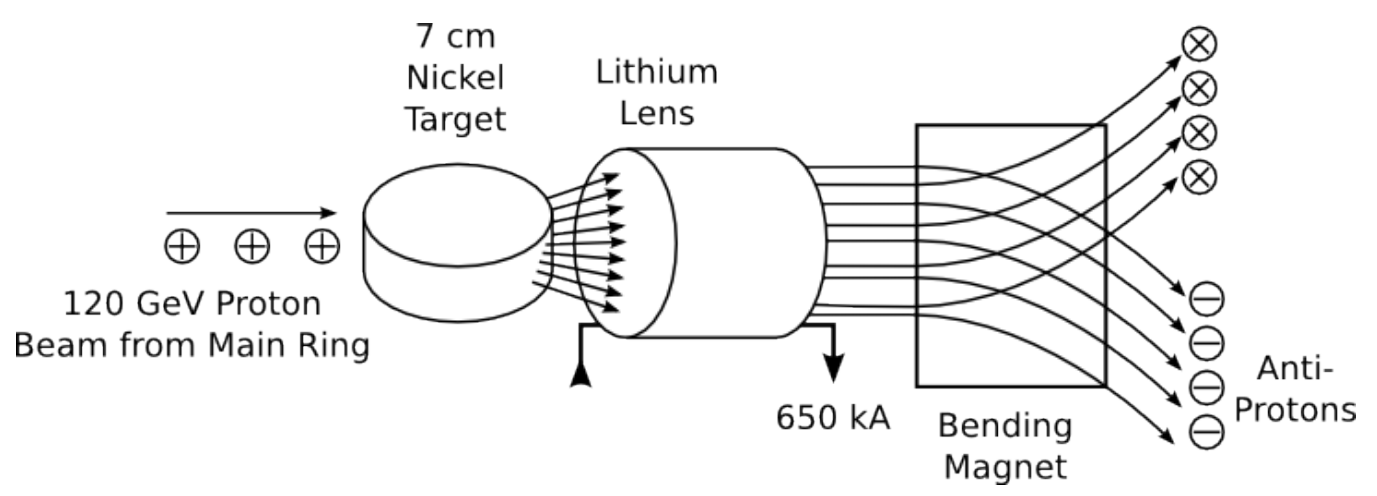

Figure 2.5: $120 \mathrm{GeV}$ protons are fired at a nickel target. The emerging particle stream is focused by a lithium lens. The anti-protons are then extracted by a bending magnet.

The anti-protons are sent to the Debuncher. Although now focused and purely negative, the energy distribution is significantly broadened. This synchrotron, shaped like an odd triangle, 
accelerates the slowest particles and decelerates the fastest. The relativistically boosted $\bar{p}$ sit upon an orbit determined by their momentum so that they see slightly different phases of the RF field. This effect causes a reduction in the spread of energies, but also destroys the bunch structure.

After the Debuncher the anti-protons are stored in the Accumulator, yet another synchrotron, which separates the anti-protons into bunches at an energy of $8 \mathrm{GeV}$. Since the anti-protons from the Target Station are produced with a wide array of angles as well as positions, a method of stochastic cooling [5] corrects the orbits of the anti-protons at this stage. In stochastic cooling, the anti-protons in the bunch produce an electrical signal which drives an electric kicker. Kicks are applied continuously, with a strength proprotional to the spread of the momentum of the particles. When enough anti-protons have been collected and cooled they are sent back to the Main Injector, accelerated up to $150 \mathrm{GeV}$, and sent to the Tevatron.

The production of anti-protons is a technically complex and costly procedure. At the end of a Tevatron store, a portion of the remaining antiprotons are recovered and kept at a fixed energy of $8 \mathrm{GeV}$ in the Recycler - a storage ring located along the ceiling of the main injector tunnel. The Recycler can also store anti-protons from the Accumulator, which increases the attainable luminosity over the initial Main Injector's design goals.

\subsubsection{The Tevatron}

The sixth and final synchrotron has a circumference of $6.28 \mathrm{~km}$ with 1,113 RF buckets and over 1000 superconducting magnets. The behemoth-like magnets produce fields of approximately 4 Tesla. The Tevatron accelerates the bunches of protons and anti-protons from $150 \mathrm{GeV}$ to $980 \mathrm{GeV}$ such that $p \bar{p}$ collisions occur at a center-of-mass energy of $1.96 \mathrm{TeV}$.

The proton and anti-proton beams travel in opposite directions, sharing the same beam-pipe and magnets with two beam-interaction points along the ring. The first, B0, is the location of the CDF detector. The second, D0, is the location of the imaginatively named D $\varnothing$ detector.

The beam travels in 36 bunches of approximately $3 \times 10^{11}$ protons each and $6-10 \times 10^{10}$ anti-protons. They are grouped into three "superbunches." Those superbunches are separated by $2.6 \mu \mathrm{s}$, whereas each bunch within a superbunch is separated by 396 ns. A full revolution takes $21 \mu \mathrm{s}$.

There have been three distinct Tevatron operating periods: Run I (1991-1996), Run IIa (2001-2006) and Run IIb (2006-present). Table 2.1 presents typical operating parameters for each of the periods. 


\begin{tabular}{|l|c|c|c|}
\hline \hline Parameter & Run I & Run IIa & Run IIb \\
\hline Beam Energy & 900 & \multicolumn{2}{|c|}{980} \\
\hline Bunches & 6 & \multicolumn{2}{|c|}{36} \\
\hline Protons/Bunch & $2.3 \times 10^{11}$ & $2.7 \times 10^{11}$ & $3.0 \times 10^{11}$ \\
\hline Anti-Protons/Bunch & $5.5 \times 10^{10}$ & $3.0 \times 10^{10}$ & $7.0 \times 10^{10}$ \\
\hline Bunch Spacing $(\mathrm{ns})$ & 3500 & \multicolumn{2}{|c|}{396} \\
\hline Peak Inst. Lumi. $\left(\mathrm{cm}^{-2} \mathrm{~s}^{-1}\right)$ & $1.6 \times 10^{31}$ & $1.5 \times 10^{32}$ & $3 \times 10^{32}$ \\
\hline $\int \mathcal{L}\left(\mathrm{pb}^{-1} /\right.$ week $)$ & 3.2 & 17.3 & 50 \\
\hline \hline
\end{tabular}

Table 2.1: Tevatron Operating parameters for Run I, Run IIa, and Run IIb.

\subsection{The DØ Detector}

\subsubsection{Luminosity}

As mentioned in Section 1.3, luminosity $(\mathcal{L})$ is a measure of the number of inelastic $p \bar{p}$ collisions per unit time and area. It is defined by Equation 2.1.

$$
\mathcal{L}=\frac{N_{p} N_{\bar{p}} n_{B} f}{2 \pi\left(\sigma_{p}^{2}+\sigma_{\bar{p})}^{2}\right.} F\left(\sigma_{l} / \beta^{*}\right)
$$

Here, $N_{p}\left(N_{\bar{p}}\right)$ are the number of protons (anti-protons) per bunch, $n_{B}$ are the 36 bunches, $f$ is the revolution frequency of the bunches, $\sigma_{p}\left(\sigma_{\bar{p}}\right)$ is the transverse size of the proton (anti-proton) beam at the collision point, and $F\left(\sigma_{l} / \beta^{*}\right)$ is the form factor that depends on the bunch length $\sigma_{l}$ and $\beta^{*}$. Given that $\sigma_{l}$ and $\beta^{*}$ for each bunch are unknown, the luminosity is measured by counting the coincident particles on the Luminosity Monitors (LM) [6, 7].

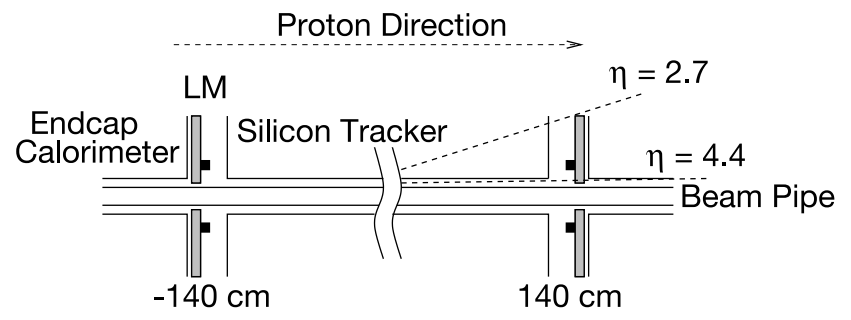

Figure 2.6: The LM detectors are located near the beam pipe on each side of the detector.

The production rate $(\mathcal{R})$ of any process can be expressed in terms of the instantaneous luminosity and its production cross section $(\sigma)$ using Equation 2.2.

$$
\mathcal{R}=\mathcal{L} \times \sigma
$$


The LM at D $\varnothing$ consist of two scintillator monitors located near the beam pipe, one on each side of the detector, covering the pseduo-rapidity region $2.7<\left|\eta_{\text {det }}\right|<4.2$. The vast majority of $p \bar{p}$ collisions produce low transverse momentum $\left(p_{T}\right)$ particles with small scattering angles which are detected by the LM. It has a time resolution of $\leq 2.7 \mathrm{~ns}$, which allows it to differentiate between particles from the interaction region which are co-incident on the faces of both monitors, and those from the beam halo which are time-separated.

The luminosity is determined from the average number of inelastic collisions per beam crossing $\left(\bar{N}_{L M}\right)$. The number of crossings with and without hits follows a Poisson distribution. From the fraction of beam crossings with no collisions we can determine $\bar{N}_{L M}$. The luminosity is given by Equation 2.3, using the beam crossing frequency $(f)$ and the effective inelastic cross section $\left(\sigma_{L M}\right)$ of the inelastic collisions [8].

$$
\mathcal{L}=\frac{f \bar{N}_{L M}}{\sigma_{L M}}
$$

\subsubsection{Tracker}

The tracking of charged particles at $\mathrm{D} \varnothing$ is performed by the two innermost detectors. The Silicon Microstrip Tracker (SMT) is located directly on the beam-pipe, with the Central Fiber Tracker (CFT) around it. Both are housed within the central magnetic field of a superconducting solenoid magnet. The field curves the trajectory of the charged particles, allowing for measurements with excellent momentum and charge resolution. These measurements impact the reconstruction resolution of primary vertices, lepton $p_{T}$, jet $E_{T}$, and make possible an $E / p$ calibration of the calorimeter using the ratio of the calorimeter energy and the tracker momentum.

The solenoid was added between Run I and Run II and was designed to optimize $\delta p_{T} / p_{T}$ given the size constraints of the calorimeter. It is $2.73 \mathrm{~m}$ long, $1.07 \mathrm{~m}$ in inner diameter, $1.42 \mathrm{~m}$ in outer diameter, and generates a field of 1.92 Tesla.

\section{Silicon Microstrip Tracker}

As the innermost detector, the SMT is crucial to the proper determination of vertices over the $\approx 25 \mathrm{~cm}$ long interaction region. It is made of strips of doped silicon semiconductors, each strip approximately $50 \mu \mathrm{m}$ in pitch. As a charged particle passes through the positive-negative $(p-n)$ junction it forms electron-hole pairs. A voltage is applied to the semiconductor, so that the charge collects onto one side of the wafer where it can be digitized and read out.

The physical layout of the detector has been selected so that tracks are perpendicular to the detector over large ranges of $\eta$. The detector is symmetric around $z=0$ (See Figure 2.7 and Figure 2.8). There are three barrel modules on either side, each with four concentric layers. They are centered at $|z| \simeq 6.2,19.0$, and $31.8 \mathrm{~cm}$. The barrel ends are all capped by disk modules, called "F-Disks." There are three additional such F-Disks located at higher $z$. The 
F-Disks are positioned at $|z| \simeq 12.5,25.3,38.2,43.1,48.1$, and $53.1 \mathrm{~cm}$. In Run IIa there were an additional two disks, called "H-Disks," which extended the $|\eta|$ coverage of the detector. The H-Disks used to be located at $|z| \simeq 100.4$ and $131.0 \mathrm{~cm}$. An additional inner layer (Layer 0) was added for Run IIb, and the H-Disks were removed, although their read-out electronics remain in support of Layer 0 .

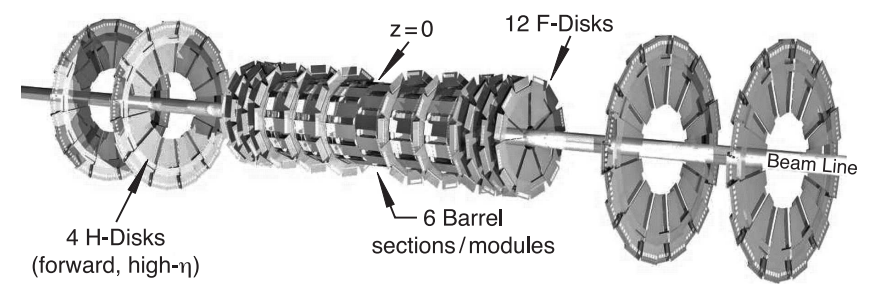

Figure 2.7: Layout of the SMT detector with the H-Disks prior to their removal for Run IIb.

Layer 0 was added in the spring of 2006 to cope with detector aging and to further improve the $p_{T}$ and impact parameter measurement of charged particles. It is crucial that these values be well measured in the increased instantaneous luminosity environment of Run IIb to maintain high quality tracking and pattern recognition. Layer 0 is mounted on a very stiff, low mass, carbon fiber composite structure which minimizes the amount of additional material in front of the calorimeter [9].

There is a mix of Single-Sided (SS), Double-Sided (DS), and Double-Sided Double-Metal (DSDM) detectors in use. Layers 2 and 4 all use DS detectors with a $2^{\circ}$ stereo angle between the strips of the two sides. The four center-most barrels use DSDM detectors on layers 1 and 3 with a $90^{\circ}$ offset between sides. The two remaining barrels use SS detectors for layers 1 and 3. The F-Disks are all DS wedge detectors, with a $30^{\circ}$ stereo offset between the sides. The H-disks had back-to-back SS wedge detectors; giving them an effective stereo angle of $15^{\circ}$. The Layer 0 detector uses a SS geometry.

Because of their orientation, barrel detectors measure $r-\phi$, whereas disks measure $r-z$. This means that particles with high $\eta$ which cross multiple disks have the best $r-z$ resolution. Each barrel has at least one side with sensors running parallel to $z$ and it is the stereo design of the double sided detectors which allow three dimensional reconstruction.

\section{Central Fiber Tracker}

The most significant contribution to the momentum resolution for tracks with $|\eta| \lesssim 1.7$ comes from the CFT (See Figure 2.8). It is made out of scintillating fibers mounted on eight concentric support cylinders from $20<r<52 \mathrm{~cm}$. The presence of the H-Disks limits the two inner cylinders to $1.66 \mathrm{~m}$ long, whereas the six outer cylinders are $2.52 \mathrm{~m}$.

Each cylinder carries four layers of scintillating fibers. The cores are made of polystyrene doped with paraterphenyl, an organic fluorescent dye. Two nested layers of fibers always run axially along $z$, and the other two layers run at angles of $+3^{\circ}$ ( $u$ layer) and $-3^{\circ}(v$ layer) 


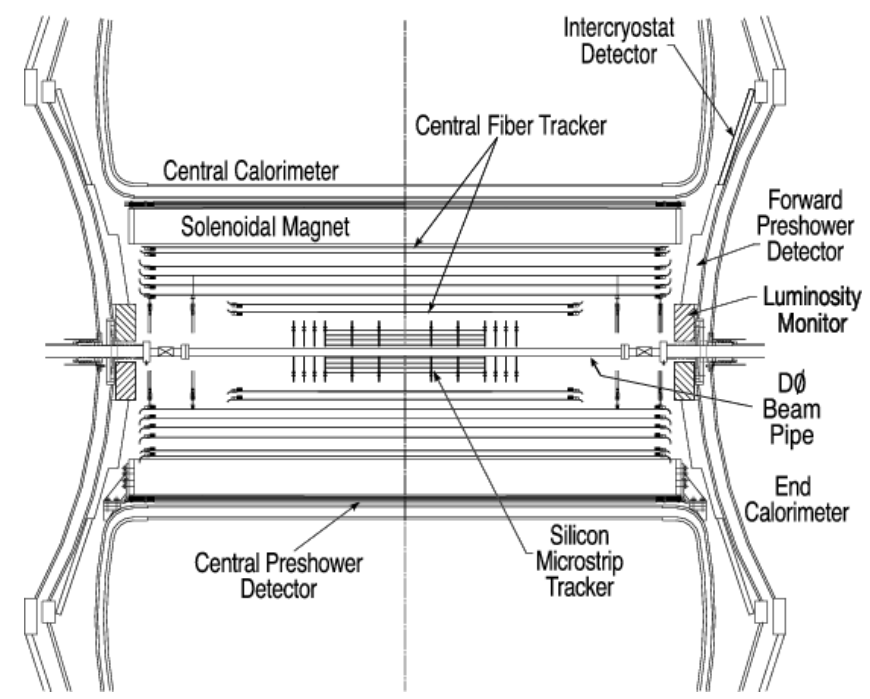

Figure 2.8: Cross-sectional view of the SMT and CFT detector systems.

in $\phi$. The scintillating fibers are $835 \mu \mathrm{m}$ in diameter. With four layers and the position of each of the fiber centers known to within $50 \mu \mathrm{m}$, the tracker has a resolution of approximately $100 \mu \mathrm{m}$. Each fiber is coupled to a waveguide, which has the same structure and, aside from the scintillating dyes, is almost chemically identical to the scintillating fibers. All the fibers have a multi-clad structure consisting of a core surrounded by two outer walls. The refractive index of the core is $n=1.59$, that of the two outer walls is $n=1.49$ (inner) and $n=1.42$ (outer). The fibers are almost completely internally reflective and the end opposite the waveguide is capped using a $90 \%$ reflective sputtered aluminum coating.

Charged particles traveling through the CFT excite the polystyrene core. This energy is transferred to the fluorescent paraterphenyl which emits light with a wavelength peaked around $340 \mathrm{~nm}$. The waveguides direct the light from within the scintillating fibers to visible light photon counters (VLPC). The VLPCs are impurity-based avalanche photodetectors, sensitive enough to detect a single photon. From here, the signal is fed to analog front-end boards (AFE), which provide pre-amp services, temperature control of the VLPCs, and Level 1 trigger information (Triggering is further described in Section 2.3).

\subsubsection{Preshower}

Because of the extra material added to the detector in Run II, mainly due to the solenoid, a significant fraction of a particle's energy is deposited before entering the calorimeter. The pre-shower detector is used both to improve triggering and to correct the energy measurements for these losses. Generally speaking, it is a hybrid tracker/calorimeter made of a combination of absorbers and scintillating strips.

There are three components to this detector. The Central Preshower (CPS), which covers 
$|\eta|<1.3$, is inside the $5 \mathrm{~cm}$ gap between the solenoid and the Central Calorimeter (CC). The two Forward Preshower (FPS) systems, which cover $1.5<|\eta|<2.5$, are mounted on the spherical heads of the Endcap Calorimeter (EC) at the North and South ends between the luminosity monitor and the inner edge of the Inner Crystat Detector (ICD). The coverage roughly matches that of the calorimeter.

The CPS and FPS are both made of triangular scintillating strips, wedged side-by-side (See Figure 2.9) to form a plane with minimal dead areas. Since particles typically pass through more than just one strip, this design improves resolution by using strip-to-strip interpolations. Similarly to the CFT, the strips are made of polystyrene plastic, doped with 1\% paraterphenyl and 150 parts per million of diphenyl stilbene. Each of the triangular strips is optically isolated by wrapping it in aluminized mylar and the ends are painted white to further enhance internal reflectivity. The strips have embedded wavelength shifting (WLS) fibers, similar to those used in the CFT. The light produced by a charged particle is transported along clear light-guide fibers, and then to VLPC cassettes for read-out.

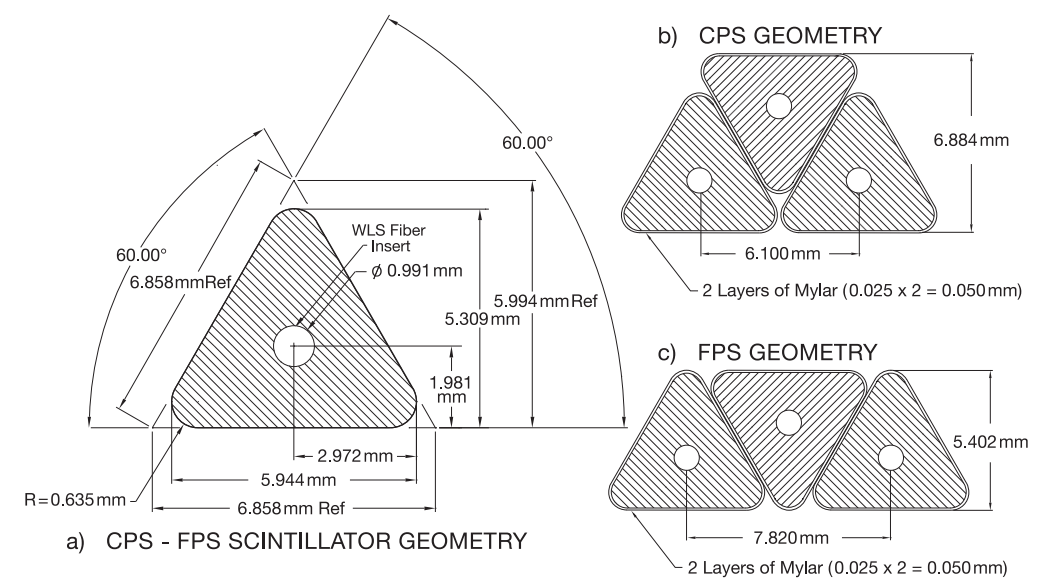

Figure 2.9: Cross section and layout geometry of the scintillator strips used in the CPS and FPS. The circle at the center is the waveguide. The hatched area is the scintillating material.

The CPS is made up of three concentric cylindrical layers, each with 1280 strips. The layers are oriented axially (along $z$ ), and at stereo angles of $\pm 23.774^{\circ}$ (the $u$ layer), and $\pm 24.016^{\circ}$ (the $v$ layer). The ends of the $u$ and $v$ layers are aligned along the end of the axial layer (See Figure 2.10a). All three are fixed on a lead radiator which is approximately one radiation length in depth for EM objects.

The FPS (See Figure 2.10b) is a sandwich of two layers of scintillating strips, a $2 X_{0}$ thick lead-stainless-steel absorber, and another two layers of strips. The layers on the low $z$ side of the absorber are called the Minimum Ionizing Particle (MIP) layers, those on the high $z$ side are called the shower layers. Due to the position of the solenoidal magnet, the MIP layers only cover $1.65<|\eta|<2.5$, whereas the shower layers cover $1.5<|\eta|<2.5$. Both the MIP and shower planes are configured in stereo $u$ and $v$ layers, with an angle of $22.5^{\circ}$ between them. For 
charged particles, the MIP layer will measure the position and direction of its track, whereas the shower layers will measure the electron and photon showers formed in the absorber. Photons, which leave no track in the MIP, will only be seen in the shower layers. For hadrons, which are less likely to shower in the absorber, the shower layer will measure a track, spatially matched to the one in the MIP layer.

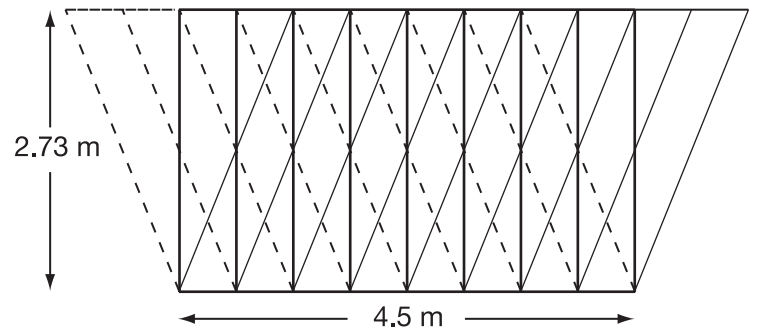

(a)

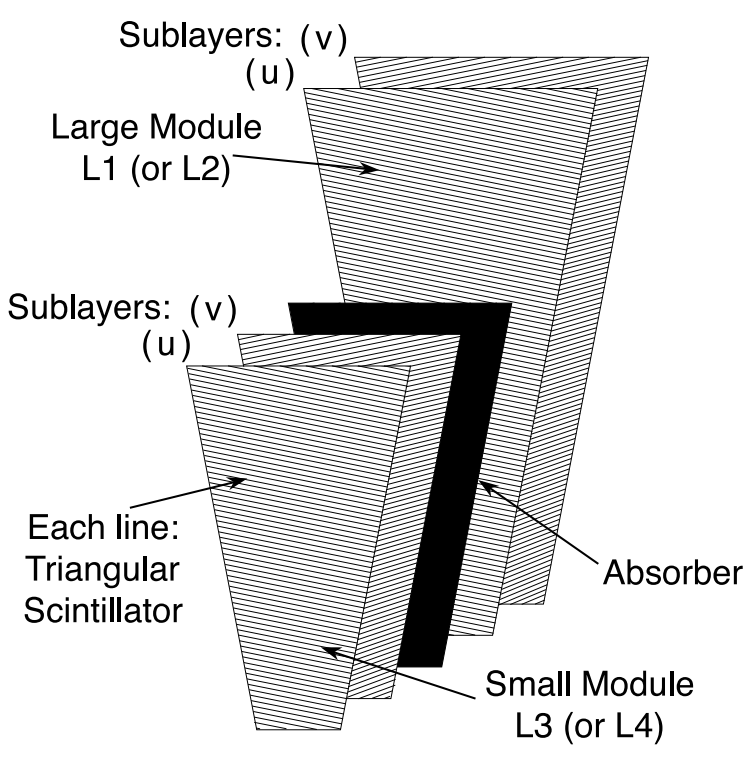

(b)

Figure 2.10: The CPS (a) is made of three concentric cylindrical layers, one oriented along $z$, the other two at stereo angles of $23.774^{\circ}$, and $24.016^{\circ}$. The FPS (b) is made of two layers of scintillating strips at angles of $\pm 22.5^{\circ}$, an absorber, and another identical set of layers.

\subsubsection{Calorimeter}

The calorimeter detects and measures the energy of electrons, photons, and hadronic jets. Additionally, it is used to detect neutrinos which leave no energy behind, but cause an energy imbalance in the detector. There is a central calorimeter (CC) with coverage over $|\eta|<1.1$ and two identical endcap calorimeters (EC) with coverage from $1.4<|\eta|<4.2$.

The fundamental building block of the calorimeter is the cell. In each cell there is a $2.3 \mathrm{~mm}$ Liquid-Argon filled gap with a grounded absorber plate on one side, and a signal board with a positive 2 to $2.5 \mathrm{kV}$ voltage on the other (See Figure 2.11). Showers are produced in the absorber plates. Shower particles ionize the atoms in the Liquid Argon gap, producing a charge which is collected on the read-out board. The thickness of the gap has been chosen so as to detect the signature of minimum ionizing particles while avoiding construction complications.

The CC and EC have three distinct layers: 


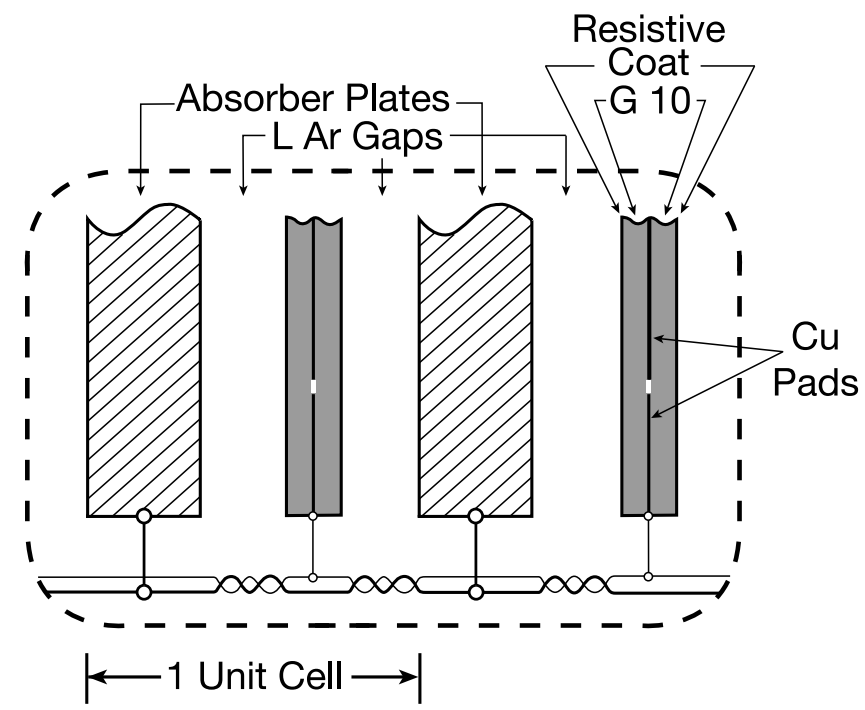

Figure 2.11: In the calorimeter cells, showers begin in the absorber plate. They ionize the Liquid Argon atoms, producing a charge which is collected on the read-out plates. The $r$ axis in the CC runs horizontally along this figure.

- one electromagnetic (EM) section with four sub-layers using uranium absorber plates.

- a finely segmented hadronic section $(\mathrm{FH})$ with three sub-layers using a niobium-uranium alloy.

- a coarsely segmented hadronic layer $(\mathrm{CH})$ also with three sub-layers made of copper in the $\mathrm{CC}$ and stainless steel in the EC.

The location of the CC layers, as well as the EC's inner, middle, and outer layers, is shown in Figure 2.12b. Each of their dimensions is motivated by the way in which electrons, photons, and hadrons deposit their energy.

Electrons and photons primarily lose their energy through bremsstrahlung $(e \rightarrow e \gamma)$ and pair production $\left(\gamma \rightarrow e^{+} e^{-}\right)$respectively. As an EM particle travels through the detector a shower forms as these two processes feed off each other. The effective energy loss of electrons is described by Equation 2.4, where $E_{0}$ is the original energy, $X_{0}$ is the radiation length of the material, and $x$ is the distance traveled by the particle. $X_{0}$ is also $7 / 9$ of the mean free path for pair production by a high-energy photon.

$$
E=E_{0} e^{-x / X_{0}}
$$

The first and second EM layers in the CC, where the pion and photon shower shapes differ the most, are $1.4 X_{0}$ and $2.0 X_{0}$ respectively in length. The third layer, which is where the 


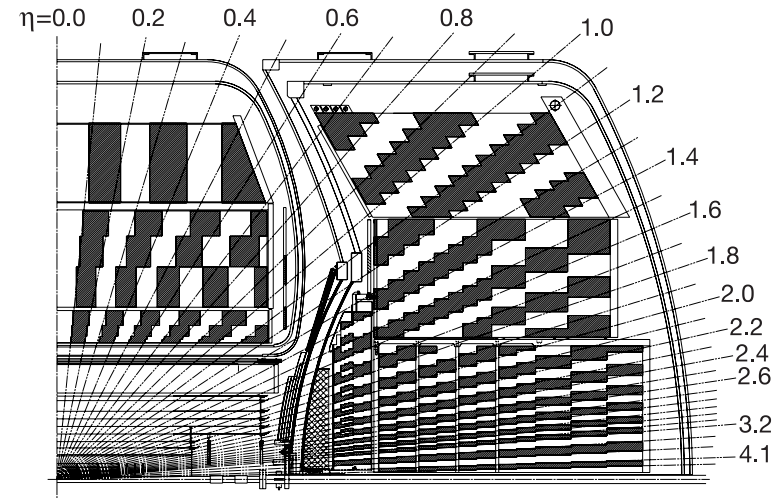

(a)

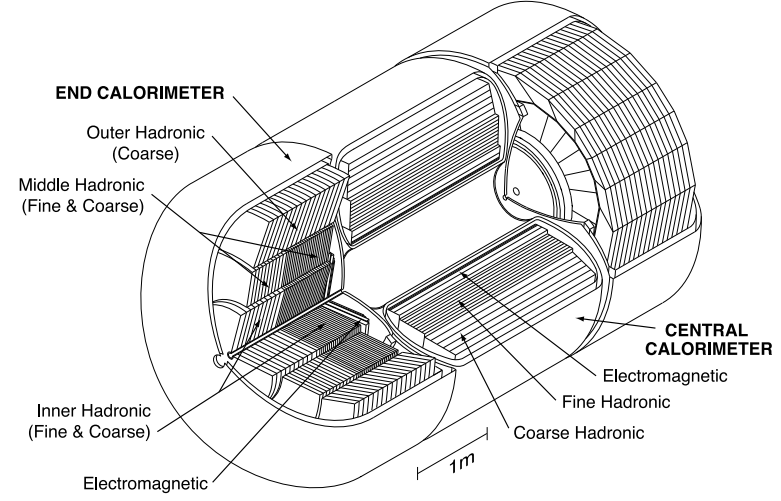

(b)

Figure 2.12: The pseudo-projective towers of calorimeter cells are shown in the sketch in the transverse plane in (a). The layout of the EM, FH, and $\mathrm{CH}$ layers is shown in the cutaway view in $(b)$.

energy shower peaks, is $6.8 X_{0}$. The fourth and final EM layer is $9.8 X_{0}$. In the EC, the layers are 1.6, 2.6, 7.9 and $9.3 X_{0}$ thick.

Hadronic particles, such as pions and kaons, primarily lose their energy through strong force interactions with the nuclei of the calorimeter material. This produces neutral and charged pions which decay into photons and more charged pions. Because of this, hadronic showers tend to be wider and longer than those produced by EM particles. For these, the unit of measure is the absorption length $\left(\lambda_{A}\right)$, which is the mean free path of a particle before it has an inelastic collision with a nucleus. The absorption lengths for each of the layers are as follows:

- The EM layers are $0.76 \lambda_{A}$ in the $\mathrm{CC}$ and $0.95 \lambda_{A}$ in the EC.

- The FH layers in the CC are 1.3, 1.0 and $0.76 \lambda_{A}$ and the the $\mathrm{CH}$ layer is $3.2 \lambda_{A}$

- All four inner FH layers in the EC are $1.1 \lambda_{A}$ and the $\mathrm{CH}$ layer is $4.1 \lambda_{A}$

- All four middle FH layers in the EC are $0.9 \lambda_{A}$ and the $\mathrm{CH}$ layer is $4.4 \lambda_{A}$

- the outer $\mathrm{CH}$ layer is $6.0 \lambda_{A}$

The transverse size of the cells has been picked to be of the same order as the size of the showers. For EM objects this is 1 to $2 \mathrm{~cm}$; for hadronic objects it is closer to $10 \mathrm{~cm}$. The cells are $\frac{2 \pi}{64} \times 0.1$ in $\Delta \phi \times \Delta \eta$ except in the third EM section where the segmentation is doubled. The cells are aligned as "towers" such that their centers lie along the $\eta$ projecting from the origin with increasing depth. Their sides however are perpendicular to the absorber plates. The CC is built in $\phi$-modules. There are 32 such modules in the EM region, each two towers 
in width. For the FH and $\mathrm{CH}$ there are 16 modules. Since there is dead space between the modules, often referred to as the $\phi$ module cracks, the energy of particles which pass through here is less well measured. Only the middle and coarse hadronic rings of the EC are built in $\phi$-modules, mitigating this problem for $\eta>1.4$.

The drift time in the cell is approximately $420 \mathrm{~ns}$. This is longer than the $396 \mathrm{~ns}$ bunch separation and leads to a "pile-up" of energy from the previous event, which must be dealt with by the read-out electronics. The charge collected on the cell pads is fed along coaxial cables into a preamplifier system. From there it is filtered and shaped using Base Line Subtraction (BLS) boards which partially fix the pileup problem. Whether or not the signal is eventually stored depends on the trigger system described in Section 2.3.

There is another detector in the gap between the $\mathrm{CC}$ and $\mathrm{EC}$ called the Inner-Cryostat Detector (ICD). The material composition varies in this region due to the cryostat walls, stiffening rings, and module end plates. The ICD is meant to correct for the energy deposited in this poorly instrumented region. Scintillation counter arrays are located in front of the surface of each EC. Each has 384 scintillator tiles of $0.1 \times 0.1$ in $\Delta \eta \times \Delta \phi$. The light produced by the tiles is collected by wavelength shifting fibers and is read out by $1.3 \mathrm{~cm}$ photomultiplier tubes (PMT).

There are also single cell readout calorimeter cells, called Massless Gaps located inside the central and end cryostat. These are essentially calorimeter cells where the cryostat itself is used as an absorbing plate.

\subsubsection{Muon}

As minimum ionizing particles, muons only deposit a fraction of their energy $(\approx 2.5 \mathrm{GeV})$ in the calorimeter. The muon detector therefore is located outside the calorimeter as the outermost detector system. There are two components to the muon detector. The central system has been in place since Run I and covers the region of $|\eta| \lesssim 1$. A new forward system was installed before the start of Run II covering $1 \lesssim|\eta| \lesssim 2$. The slight ambiguity in $\eta$ coverage is because the detector is rectangular.

Toroid magnets lie between the two innermost muon detector layers. There is a central toroid producing a magnetic field of $1.8 \mathrm{~T}$, and two forward toroids (North and South) each producing a field of $1.9 \mathrm{~T}$. These magnets curve the trajectory of the muons to allow for $p_{T}$ and charge measurements by the muon detector independent of the tracker.

Proportional Drift Tubes (PDT) were used in the central detector. These are filled with a gas mixture of $84 \%$ Argon, $8 \% \mathrm{CF}_{4}$, and $8 \% \mathrm{CH}_{4}$. As a charged particle passes through a PDT it ionizes the gas. The charge is collected by high-voltage wires which run along the center of the tube, and the position is extrapolated from the time of charge collection on multiple wires. The new forward detector uses Mini Drift Tubes (MDT), which are filled with a gas mixture of $90 \% \mathrm{CF}_{4}$ and $10 \% \mathrm{CH}_{4}$. An iron-polyethylene-lead beam-pipe cover in the forward region reduces background muons from the beam-halos. 
In both the central and forward regions there are three drift tube layers; The first one is located inside the toroidal magnet (the A Layer), and the two others (the B and C Layers) are located outside. There are two layers of pixel scintillators affixed to the central A-Layer (the A- $\phi$ counters) and around the outside of the central B and C Layers (the cosmic cap and bottom counters respectively). There is a hole in the bottom coverage of the central muon system to make space for support structures, from $225^{\circ}<\phi<310^{\circ}$. The A Layer has four sub-layers of PDT (except in the bottom hole where it only has three), whereas the B and C layers each have three sub-layers.

The three forward layers (also denoted A, B, and C, and with the same number of sub-layers) are set between scintillator pixel detectors (the trigger scintillation counters). Although the MDT have somewhat better time resolution than the PDT, at 4 ns the scintillation counters remain significantly better. The scintillation counters in the central and forward regions have good enough resolution to associate a track to a drift tube for a particular bunch crossing, and can be used to veto cosmic muons. Generally speaking, the scintillation counters are used for identification and triggering, whereas the wire chambers are used for precise positioning as well as additional triggering.

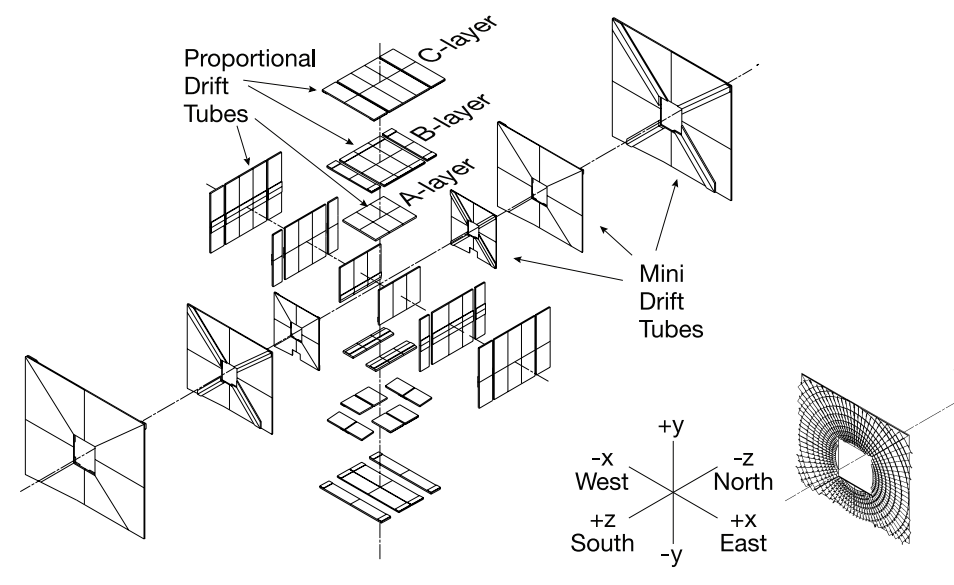

(a)

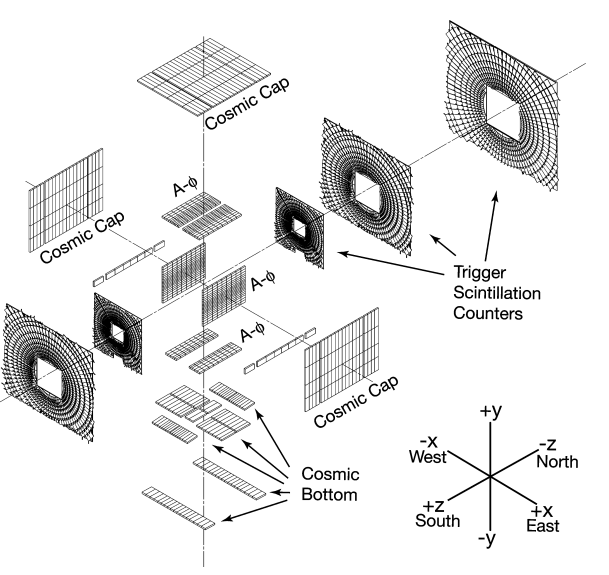

(b)

Figure 2.13: An exploded view of the muon system showing the drift tube system in (a) and the scintillating system in (b).

\section{$2.3 \quad$ Triggering}

Due to financial and technical constraints, it is impossible to record every collision produced at the heart of the $\mathrm{D} \varnothing$ detector. The final rate at which events are written to tape is required to be $\approx 100 \mathrm{~Hz}$, and at a collision rate of $1.7 \mathrm{MHz}$ the task of recording and storing events imposes a major bottleneck. The rate is whittled down by a three level trigger system which grows in 
sophistication and processing time per event at each stage. At Level 1 (L1) the rate is reduced from $\approx 1.7 \mathrm{~Hz}$ to $\approx 2 \mathrm{kHz}$ by custom made hardware and firmware. This hardware has been designed with a large enough buffer to make this level deadtime-less. Level 2 (L2) consists of Single Board Computers (SBC) which parse information from each subdetector using specialized algorithms. At L2, primitive variables are constructed from the output of multiple detectors to give it the ability to make requirements on the event topology. This further reduces the rate to $\approx 1 \mathrm{kHz}$. Finally, Level 3 (L3), which is run on a farm of computers, uses sophisticated algorithms analogous to those found at the analysis level to reduce the rate to $\approx 100 \mathrm{~Hz}$. A trigger is defined as the AND of L1, L2, and L3 terms. Events which satisfy the 3 terms are written to tape for offline reconstruction, which we treat in detail in Chapter 4. Certain triggers would still exceed the target rate to tape if accepted every time they fired, especially at higher operating luminosities. For this reasons, triggers can be assigned a prescale, defined over a luminosity range, where the system randomly rejects 1 of of every $\mathrm{N}$ events.

\subsubsection{The Level 1 Trigger}

Data from the CFT, Preshower, Calorimeter, and Muon systems are all used at L1. L1 operates on Field Programmable Gate Arrays (FPGA) which are capable of calculating a trigger term in $132 \mathrm{~ns}$. A $4.2 \mu \mathrm{s}$ buffer stores the trigger terms long enough to allow for deadtime-less operation. To keep the data transfers small, only a reduced set of information is available at L1. The 128 different physics conditions triggers and their prescales are managed by the Trigger Framework (TFW).

Level 1 Calorimeter (L1CAL) trigger decisions are based on the $E_{T}$ deposits in 1280 trigger towers of $\Delta \eta \times \Delta \phi=0.2$. Triggers specify threshold levels in a set number of trigger towers for the Electromagnetic (EM) layers or the EM plus Hadronic $(\mathrm{H})$ layers. L1CAL triggers may also make requirements on the sum of the transverse energy in a $4 \times 8$, in $\eta \times \phi$, cluster of trigger towers, the sum of all the $E_{T}$ in the event, as well as on the $\mathbb{E}_{T}$. A method of "Sliding Windows" was introduced in Run IIb. Regions of interest are defined by looking for trigger tower clusters in fixed-sized $(2 \times 2$ in $\eta \times \phi)$ windows to find local maxima. A trigger is defined by requiring that the energy distribution around the local maximum satisfies some threshold conditions [10].

Level 1 Central Track Trigger (L1CTT) provides the ability to trigger on reconstructed trajectories using the CFT, FPS, and the axial CPS layers. Track candidates are identified from hit patterns in the CFT by comparing them to 20,000 predefined tracks. The selected track candidate is spatially matched to energy deposits in the PS and placed into 4 energy bins ranging from 1.5-3, 3-5, 5-10, and $>10 \mathrm{GeV}$. Trigger requirements are made on the number of tracks above $p_{T}$ threshold for tracks with and without a matching PS cluster. 
Level 1 Calorimeter Tracks (L1CALTRACK) substantially reduces trigger rates from background sources by matching L1CTT tracks to L1CAL jets and electrons. The spatial match is made with L1CTT tracks which satisfy some threshold transverse momentum values, as well as some track isolation and preshower criteria.

Level 1 Muon (L1MU) finds muon candidates using information from the wire chambers, muon scintillation counters, and tracks from the L1CTT. The muon system is divided into Central, North, and South regions. Candidates are formed which satisfy a variety of quality conditions based on matches in the scintillators, as well as minimum $p_{T}$ conditions.

\subsubsection{The Level 2 Trigger}

The Level 2 (L2) trigger system's algorithms are more sophisticated, and hence slower, than those used in L1. They quickly approximate physics objects using a parsed set of data from the tracking, calorimeter, preshower and muon systems. The physics objects are formed in parallel for each subdetector. The physics objects are then used by a global processor (L2GBL) which examines the event topology.

Level 2 Calorimeter (L2CAL) expands its purview to all 2560 calorimeter trigger towers. Jets are identified by clustering towers into $5 \times 5$ groups around seed towers with EM + Hadronic (TOT) energy $\geq 2 \mathrm{GeV}$. Electrons and photons are formed using a similar list of seed towers where the EM energy is $\geq 1 \mathrm{GeV}$. EM clusters are defined using the seed tower and its neighbor with the largest $E_{T}$. The EM fraction is calculated using the EM and TOT layers, whereas $3 \times 3$ arrays of towers are used to calculate isolation. The $\mathbb{E}_{T}$ is calculated from the energy deposited in the EM and $\mathrm{H}$ layers. The jet, electron, photon, and $\mathbb{E}_{T}$ candidates are then fed to L2GBL.

Level 2 Muon (L2MU) consists of two pre-processors, one for the central region (L2MUC) and one for the forward region (L2MUF). Data from the L1MU, the drift chambers and scintillation counters pass through Second Level Input Computers (SLIC). The momentum of the candidates are measured by combining A-layer track segments with those in the B and C layers. The algorithms at L2 improve upon those at L1 by considering calibration information and better timing measurements to improve the quality of the candidates. The $\eta, \phi, p_{T}$, and sign of the L2 candidates are sent to L2GBL.

Level 2 Preshower (L2PS) is designed to find signs of early showering from electrons or photons. It looks for these objects in the axial and stereo layers of the CPS, as well as in the FPS. The algorithm derives $\eta$ and $\phi$ coordinates for clusters in the PS and matches these to calorimeter trigger towers. Any hits in a $\Delta R<0.5$ window around the trigger tower are assigned to it. 
Level 2 Silicon Track Trigger (L2STT) improves the measurement of the L1 tracks by using additional hit information in the SMT. The additional constraints from SMT hits help to reject artificial L1 triggers produced by fake track patterns. Trigger level tagging of long-lived particles, such as $B$ hadrons, is made possible by the improved spatial resolution.

Level 2 Central Track Trigger (L2CTT) considers tracks from L1CTT and L2STT in two $p_{T}$ sorted lists. For the L1CTT tracks, additional hit information is used to refine the measurement. Angular values at the interaction point and in the third EM calorimeter layer are calculated before passing the tracks to L2GBL. These values help calculate trigger level cuts on track isolation.

\subsubsection{The Level 3 Trigger and Data Acquisition}

Level 3 (L3) triggering performs a partial reconstruction of physics objects. This incurs a high computational cost and L3 is therefore run on a farm of nodes ${ }^{1}$ rather than on specialized hardware. The data acquisition system (L3DAQ) conducts the transfer of data from the read-out crates to the L3 trigger farm via Ethernet. The L3 trigger system uses the full detector read-out and runs reconstruction algorithms similar to those used in offline event processing (discussed in Chapter 4). These triggers are able to select candidate events using correlations between objects, such as their invariant mass or angular separation. Each type of object is reconstructed using a software algorithm called a filter tool. These algorithms perform the bulk of the work: unpacking raw data, locating hits, forming clusters, applying calibration, and reconstructing the electrons, muons, taus, jets, vertexes and $\mathbb{E}_{T}$.

Events which satisfy the L3 trigger requirements are written to permanent storage and are eventually passed through the full offline object reconstruction.

\footnotetext{
${ }^{1}$ There are now over 400 nodes in the L3 farm.
} 


\section{Chapter 3}

\section{Monte Carlo Simulation}

"Stop! Hoo, halte! Ne plus utiliser cette porte-ci au'jourd'hui. Vous sortez par l'arriére... éxperience en cours!"

\subsection{Event Generators}

The physics we expect to observe in the detector is simulated by Monte Carlo (MC) modeling. MC methods work by repeatedly modeling physical processes and varying the initial conditions within their statistical parameters. Each throw of the dice produces a new outcome; taken as a whole, they provide statistical predictions which help us to understand the collected data. Whereas processes of interest happen only very rarely in the real-world collisions, the $\mathrm{MC}$ collisions produce them each time. The MC yields are normalized to the Data using the appropriate cross sections and luminosity, allowing us to make predictions about the behaviour of our signal and backgrounds with much less statistical jitter than in the Data.

The MC in this analysis were produced using two generators: Pythia [11] and AlPGEN [12]. These programs are designed to model the physics interactions which produce the events from their inception through their various decays. This includes, but is not limited to:

- Initial State Radiation (ISR) in which the accelerated quarks undergo the reaction $q \rightarrow q g$

- The Hard Scattering in which the partons of the $p \bar{p}$ system collide

- Final State Radiation (FSR) in which the outgoing quarks also radiate gluons

- Hadronization in which jets of hadronic material are produced as quarks combine

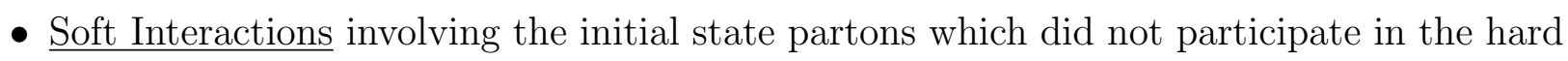
scatter 


\subsubsection{Pythia}

PythiA is a Leading Order (LO) event generator which calculates the process cross section and then uses the Parton Shower model to account for the QCD and QED radiations. Showering evolves from the initial high momentum scale $(Q)$ down to a cut-off value $\left(Q_{0}\right)$ to obtain the final state partons. Color confinement, which causes hadronization (See 1.1.2), is modeled using the String Model. Strings connect each quark/anti-quark $(q \bar{q})$ pair. Each string carries a potential energy which grows linearly with the length of the string until there is enough energy to snap the string. A new pair of quarks $\left(q^{\prime} \bar{q}^{\prime}\right)$ is produced, connected to the two torn remnants of the original string. This process continues until only on-shell hadrons remain. The DØ MC produced with PYTHIA use the CTEQ6L1 [13] parton distribution functions (PDFs) to describe the initial beam particles - protons and anti-protons in this case.

As it turns out, this model provides a fairly accurate description of the jet sub-structure, but leaves something to be desired in multi-jet events.

\subsubsection{Alpgen}

The second MC generator, ALPGEn (also a LO event generator), uses the Matrix Element method instead of the Parton Shower method. It can exactly calculate the Leading Order matrix elements for a large number of QCD and EW interactions at the parton level. This approach makes it very good at modeling multi-jet events, but it has trouble with their underlying structure. To get the best of both worlds, Alpgen is typically interfaced with Pythia where Alpgen generates the events at the parton level, and Pythia handles the showering and hadronization.

\subsubsection{MLM Matching Scheme}

To properly normalize the MC to the Data, and to avoid producing biased distributions, it is important not to double-count the events produced by the Alpgen/Pythia tag-team. The MLM Matching Scheme [14] is designed to avoid this risk in multi-jet events where there is some ambiguity in the multiplicity of the partons.

Consider the case of some final state with a boson (either a $W$ or a $Z$ ) and three jets. We wish to consider separately the two following category of events:

- The three jets were produced by three partons

- The three jets were produced by two partons, where a large angle gluon emission by one of the two produced a third jet.

Events are generated in bins of exclusive parton multiplicities $(n l p)$. When the events are fed to Pythia, the event's quarks and gluons are clustered into jets. If the event contains a jet cluster which is not matched to one of the $n l p$ partons, it is rejected. Higher multiplicity bins 
(typically above 4) are produced inclusively. In this case, the algorithm is modified to require that each of the nlp partons is matched to a jet, making no such requirement on any of the additional jets which may happen to populate the event.

\subsection{Detector Simulation}

So far we have described the methods which are used to produce the physics of interest. Since our analysis methods rely on our instrumentation, it is also important to properly model the D $\varnothing$ detector. The Pythia and AlPGEn generators both maintain a list of all particles in the event, allowing us to do just that. After having modeled the detector, as described below, the rest of the reconstruction chain is identical for the Data and the MC.

\subsubsection{Detector Material}

The passage of particles through the detector material is modeled using D0gstar (D $\varnothing$ GEANT Simulation of the Total Apparatus Response) [15]. D0gstar is based on a software package developed at CERN called GEANT (GEometry ANd Tracking) [16]. It is meant to simulate the detector response that will be obtained from the DAQ system by modeling the geometrical acceptance of the detector and the dead material. D0gstar is tasked with simulating the following effects:

- Ionization of the particles

- Showering in the detector

- Interactions with the magnetic field

- Energy deposition (i.e. hits)

\subsubsection{Electronics}

The next step is to simulate the behaviour of the detector electronics. D0sim [17] models the effects from electronic noise, pile-up from the soft underlying event (minimum bias) or additional inelastic $p \bar{p}$ collisions, and detector inefficiencies in the SMT, CFT, and muon subdetectors. The minimum bias events are actually taken from data, over a range of luminosities, and overlaid on top of the simulated event.

The rest of the reconstruction chain is identical for both the Data and the MC. 


\subsection{Simulation Corrections}

The MC remains an imperfect estimation of the data, despite the large ammount of work that has been invested into the chain of tools which simulate events. There are a number of effects, related to the modeling of the underlying physics, the detector, or the selection requirements which necessitate that we apply corrections at the analysis stage. Here we cover the corrections which have become common fare at $\mathrm{D} \varnothing$ and are used in both analyses.

- Luminosity Profile: The MC samples are overlaid with Minimum Bias events in Section 3.2.2 to model the additional detector activity in the event. Since the instantaneous luminosity decreases in a non-monotonic manner from the beginning to the end of the store, it is necessary to re-weight the MC to match the Data's luminosity profile.

- $\underline{z \text { vertex }}$ : The distribution of the position of the vertex along the $z$ direction is slightly non-Gaussian, whereas in MC the $z$ position follows an exactly Gaussian distribution. This can affect the vertex finding efficiency, so we re-weight the MC to make these two distributions agree.

- $Z p_{T}$ : Since neither Pythia or Alpgen model the $Z p_{T}$ distribution for events with $p_{T} \lesssim 100 \mathrm{GeV}$, we re-weight the $Z \mathrm{MC}$ samples as a function of the generator level $Z$ $p_{T}$. The re-weighting functions have been derived from measurements in $Z \rightarrow$ ee data. Optionally, this correction can be parametrized in bins of jet multiplicity.

- Jet Smearing, Shifting, and Removal : The formation of jets is a tricky thing to model due to both physics (such as the hadronization of the partons) and detector (such as the jet energy scale) effects. Three corrections are performed on the MC to match the data distributions.

- The jet transverse energies are multiplied by a number, randomly picked from a Gaussian distribution, so as to smear the distribution.

- The MC jets have slightly higher energies after applying the JES corrections, so the jets are shifted down by a function with respect to the jet $p_{T}$.

- The jet finding efficiency in MC is slightly higher than in data. Jets are randomly removed by picking a number from a flat distribution such that their efficiency is the same as in data.

- Lepton Smearing: Just as with the jets, the resolution of the reconstructed electrons and muons in MC is slightly sharper than in data. The MC energies are smeared such that the width of the $Z$ mass distributions agree in both Data and MC.

- Lepton Identification : The MC lepton finding efficiency is higher in MC than in Data. Rather than randomly rejecting leptons, as we did with jets, we apply a weight to the 
event to force agreement between the predicted yields. The tag-and-probe method is used to measure the lepton finding efficiency as a function of kinematic variables such as the $p_{T}, \eta, \phi$, or $P V_{z}$. The efficiencies depend on the lepton requirements made by the analysis.

- Trigger Efficiency: The MC generation chain contains no information about the triggering system since the trigger requirements are set at the analysis level. There are now a number of standardized trigger suites for which selection efficiency has been measured. Depending on the type of trigger, the efficiency has been measured against the kinematic variables of electrons, muons, jets, or combinations of each. For each candidate object, we calculate a probability that it will satisfy and fire a trigger. These probabilities are applied as weights on the MC events. 


\section{Chapter 4}

\section{Reconstruction and Identification}

"Je vais pouvir construire: ... Attends! Un orgue electronique, un detecteur de mensonge, plusieurs récepteurs radio, des émetteurs de parasites, des tas de trucs sonores, des machins qui se déclenchent a la lumiére, á la voix, á ... á ..."

In this chapter we will cover the algorithms which take the data collected in the subdetector components described in Chapter 2, and interpret them as physical objects. These are the objects which are then used in physics analyses. This stage of processing is called "Reconstruction" and is performed by the d0reco package.

\subsection{Tracks}

Tracks are reconstructed from the data taken by the SMT and CFT subdetectors. These two systems do not have the same coverage in $\eta$, resulting in three reconstruction regions of overlap, each with progressively worsening momentum resolution ${ }^{1}$ :

1. The central region $(|\eta|<1.7)$ where both the SMT and CFT are used. These tracks are typically associated to 14 to 16 hits in the CFT.

2. Tracks which are produced in the intermediate region $(1.7 \leq|\eta| \leq 2.0)$ where the CFT coverage is reduced. These tracks typically have 8 to 15 CFT hits.

\footnotetext{
${ }^{1}$ The muon $p_{T}$ is measured using the tracker, so this effect is clearly visible when we derive the muon transfer functions in Section B.1.
} 
3. The forward region $(|\eta|>2.0)$ has no CFT coverage. Tracks produced here rely entirely on the SMT detector.

Charged particles tend to deposit their energy into clusters of adjacent strips or fibers. These clusters are exploited by the two algorithms employed at D $\varnothing$ to reconstruct tracks. Both algorithms are based on a Kalman filter approach [18].

The Histogramming Track Finder (HTF) [19] applies a pattern recognition method to histograms. It is optimized for efficient reconstruction of high $p_{T}$ tracks $(\gtrsim 20 \mathrm{GeV})$ with good isolation of the sort typically observed for electrons. The rate of fakes must be kept as low as possible to provide proper separation of electrons and photons.

Track parameters are all evaluated with respect to the $(0,0,0)$ origin of the detector. Each point in $(x, y)$ space is transformed into $\left(\rho=\frac{q B}{p_{T}}, \phi\right)$ space, where $\rho$ is the track curvature and $\phi$ is the direction of the track at the position of closest approach to the beamspot. In the case of hits produced by a charged particle, the lines between $(0,0)$ and $(x, y)$ will all map to a single point in $(\rho, \phi)$ space. The detector is divided to give it a $2 \mathrm{D}$ histogram structure and in the ideal case, selecting tracks is as simple as finding the bin with the largest number of entries. A different approach is required when dealing with an environment where there is considerable noise and some tracks produce as few as 4 hits in the detector. Cells below a minimum hit multiplicity are rejected, as are those where the majority of their hits are contained in a nearby cell.

The Alternative Algorithm (AA) [20] uses a road-following methodology. It is designed to maximize computational efficiency by producing a minimal number of candidate tracks in a high energy and high luminosity environment.

AA tracks are constructed from an initial set of three hits in the SMT or three hits in the CFT which satisfy initial conditions on their curvature, angular separation, and fit $\chi^{2}$. Candidates are formed by extrapolating the track to a window in the next tracking layer. Hits in the window are added if they increase the $\chi^{2}$ by no more than 16. Any layer in which no hits are associated with the track counts as a "miss." Tracks are extended until the algorithm reaches the end of the detector, or three misses are recorded.

The list of tracks produced by the HTF and AA algorithms are fed to a Kalman filter. The filter compensates for $d E / d x$ losses and multiple Coulomb scattering effects. It determines the optimal track parameters using predictive algorithms and a method similar to a multidimensional $\chi^{2}$ minimization. Candidates which are not compatible with the predictions are removed at this stage. 


\section{$4.2 \quad$ Vertices}

The position of the $p \bar{p}$ hard scatter collision is called the Primary Vertex (PV). The proper determination of the PV is crucial to the later measurement of the direction of physical objects and magnitude of the $\mathbb{E}_{T}$. Misidentification, or misplacement, of the PV can wreak havoc on the tagging of b-jets. It is therefore important to reliably distinguish between the hard scatter vertex and the vertices from additional $p \bar{p}$ interactions not producing a hard scatter (minimum bias or MB). The algorithm used at DØ is performed in four steps [21]:

1. Tracks from Section 4.1 with $p_{T}>0.5 \mathrm{GeV}$ and $\geq 2$ SMT hits (if $|z|<36 \mathrm{~cm}$ ) are extrapolated back to the $z$-axis. Those with $\Delta z<2 \mathrm{~cm}$ along the beam line are clustered together and the vertices with the largest number of tracks are stored in a list.

2. The tracks from the $1^{\text {st }}$ step are fit to a common vertex using a Kalman filter. Those with the largest $\chi^{2}$ are iteratively removed until the $\chi^{2}$ per degree of freedom is less than 10. The location and width of the beam is determined from the distributions of the fit vertex.

3. The tracks in each cluster are sorted by their distance of closest approach (DCA) to the beam spot from the $2^{\text {nd }}$ step. Tracks with a $\frac{|D C A|}{\sigma(D C A)}>5$ are rejected and the final location of each vertex candidate is determined using a Kalman fitter.

4. Tracks from hard interactions have higher $p_{T}$ than tracks from MB interactions [22]. It is therefore possible to calculate the probability that a vertex be produced by an MB interaction (PMB) using Equation 4.1 and Equation 4.2. The vertex with the smallest $\mathrm{PMB}$ value is chosen as the $\mathrm{PV}$.

$$
P\left(p_{T}\right)=\frac{\int_{\log _{10}\left(p_{T}\right)}^{i n f} F\left(p_{T}\right) d p_{T}}{\int_{\log _{10}(0.5)}^{i n f} F\left(p_{T}\right) d p_{T}}
$$

where $F\left(p_{T}\right)$ describes the MB track $\log _{10}\left(p_{T}\right)$ spectrum distribution from MC.

$$
P M B=\Pi \sum_{k=0}^{N-1} \frac{-\ln \Pi}{k !}
$$

where $\Pi$ is the product of individual probabilities for each of the $N>0$ tracks with $p_{T}>$ $0.5 \mathrm{GeV}$ associated to the vertex.

\subsection{Muons}

To be reconstructed as a muon, a particle must leave a signature in the tracking and muon subdetectors, traversing the calorimeter along the way. Their identification is substantially less 
ambiguous than that of the electrons, photons, and jets, but only covers the region $|\eta|<2$.

There are three types of reconstructed muons at DØ [23]:

local-muons which are found in the muon wire chambers and scintillation counters and have their momentum determined from the curvature due to the magnetic field of the toroids.

central-muons are an extension of the local muons for which the momentum resolution is dominated by a track measurement in the central tracker to which the muon was spatially matched.

calorimeter-muons are identified by their minimum ionization the calorimeter.

Only the central-muons (and by extension the local-muons) will be discussed here, as they are the only type used in the two analyses described in this thesis.

Reconstruction begins by forming "segments," fitting lines from at least two hits in each layer of the muon chambers and matching them to scintillator hits. Muon candidates are initially formed by combining segments from the B and C layers. Unmatched segments are rejected and those which are kept are matched to an A layer segment. Only BC segments with an A layer match, or A layer segments without a BC match are kept. Candidates at this stage of reconstruction satisfy the local-muon definition. A track from the central tracking system is matched to the local muons if possible. Local-muons with a successful track-match are then central-muons.

The muon type (referred to as nseg) describes the various ways in which a local or central muon can be reconstructed. Positive (negative) sign indicates the presence (lack of) a track-match. An absolute value of 3 indicates the presence of hits in the $\mathrm{A}$ and $\mathrm{BC}$ layers, 2 indicates $\mathrm{BC}$ layer hits only, and 1 indicates A layer hits only. Calorimeter muons are assigned a value of nseg 0.

The purity of the muon sample is controlled by defining a quality variable. This uses the number of hits in either the SMT or CFT, the DCA, and the $\chi^{2}$ per degree of freedom (dof) of the central track fit. The three qualities are:

loose : $|D C A|<0.2(<0.02) \mathrm{cm}$ for tracks with no (at least one) SMT hits.

medium : Same as loose, requiring that the $\chi^{2} /$ dof $<4.0$.

tight : Same as medium, requiring that there be SMT hits associated with the track.

b-quarks decaying semi-leptonically can produce muons embedded in a jet. The track and calorimeter isolation of the muon can serve to reject this source of muons. Five isolation variables are defined:

TrackHalo is the scalar sum of track $p_{T}$ within a cone of radius 0.5 centered around the muon's track: $\sum^{\text {tracks }} p_{T}$ in $\Delta R<0.5$ 
CalorimeterHalo is the scalar sum of the energy deposited in calorimeter cells with an annulus of inner (outer) radius $0.1(0.4)$ centered around the muon's track: $\sum^{\text {cells }} E_{T}$ in $0.1<\Delta R<0.4$

DeltaR is the distance to the closest jet in $\eta-\phi$ space.

ScaledTrackHalo is the TrackHalo divided by the muon's $p_{T}$ : $\left|\sum^{\text {tracks }} p_{T} / p_{T}(\mu)\right|$ in $\Delta R<0.5$

ScaledCalorimeterHalo is the CalorimeterHalo divided by the muon's $p_{T}:\left|\sum^{\text {cells }} E_{T} / p_{T}(\mu)\right|$ in $0.1<\Delta R<0.4$

Cosmic ray muons can be identified by using the timing information from the scintillator hits. A veto can be applied on muons for which the hit is more than 10ns out of time in the A, B, or C layers.

\subsection{Jets}

Color confinement implies that quarks can not exist freely and must group together to form hadrons of neutral color. When a quark is separated from a hadron, for example during a hard scatter collision, new quark/anti-quark pairs are pulled out of the vacuum to dress the lone quark in a new hadron. This process, called hadronization, and the series of decays which follow it, produce "jets" of particles, typically pions, which leave traces in the central tracker and shower in the calorimeter - depositing most of their energy in the hadronic layers.

Jets are reconstructed using a cone algorithm in four steps:

1. The four-vectors of the calorimeter cells are summed together in what is called the E-Scheme to form jet towers.

$$
\mathbf{p}^{J}=\left(E^{J}, p^{J}\right)=\sum_{i}\left(E^{i}, p_{x}^{i}, p_{y}^{i}, p_{z}^{i}\right)
$$

$E$ is the energy of the cell. The direction of $\boldsymbol{p}$ is defined from the primary vertex to the center of the cell and its magnitude is the same as $E$. The towers are built in pseudo-projective layers such that each cell above threshold is within a tower, and each tower has at least one cell. Although the cell four-vector measurements are massless, a tower has a mass if the momenta of the cells are not collinear.

2. The Jet Simple Cone Algorithm builds a list of pre-clusters. It loops over a set of towers from the previous step, ordered by decreasing $p_{T}$. If a tower's highest $p_{T}$ cell is in the Coarse Hadronic layer or the End Cap Massless Gap, that cell's energy is removed from the tower sum and the set is reordered. A pre-cluster is formed by selecting the tower from the set with the largest $p_{T}$. Any unassociated towers within $\Delta R<0.3$ in $\eta-\phi$ space 
are assigned to the pre-cluster if its $p_{T}>1 \mathrm{MeV}$. As towers are associated to pre-clusters, the algorithm removes them from the set until none remain with $p_{T}>500 \mathrm{MeV}$. When all iterations over the towers have finished, any pre-cluster with $p_{T}<1 \mathrm{GeV}$ which is made of only one tower is removed from the final list of pre-clusters.

3. The pre-clusters serve as seeds for the Run II Cone Algorithm, which is used to construct proto-jets with radius 0.5 or 0.7 in $y-\phi$ space in this second to last step (where $y$ is rapidity, rather than pseudorapidity). The algorithm loops over the list of pre-clusters sorted by decreasing $p_{T}$. If the distance between the pre-cluster in question and the nearest proto-jet is greater than $\frac{R_{\text {cone }}}{2}$ the pre-cluster is marked as a proto-jet seed. All the pre-clusters within the proto-jet's cone are added, recalculating the centroid weighted mean each time. This is repeated until the change in mean is less than $\Delta R<0.001$ or the list of pre-clusters has been looped over 50 times. The proto-jet is only kept if its $p_{T}$ is greater than $4 \mathrm{GeV}$. When this process is completed, the midpoints between two proto-jets are added as seeds to remove sensitivity to soft radiation.

4. The final step is designed to remove any double counting from the Run II Cone Algorithm output. The proto-jets are ordered by decreasing $p_{T}$ and looped over. For each proto-jet the algorithm calculates the $p_{T}$ of the pre-clusters shared with any other proto-jet. If the shared $p_{T}$ is $>50 \%$ of the neighbor's $p_{T}$, the two proto-jets are merged using the E-Scheme. Otherwise, the proto-jets are split by requiring that each item be assigned only to its closest proto-jet in $\Delta R$ space and the list is reordered.

It is important to take into account the response of the calorimeter to electromagnetic and hadronic particles. The energy of the jets returned by the Run II Cone Algorithm is scaled to take it from the reconstructed energy $\left(E_{j e t}^{\text {meas }}\right)$ to one more closely matching the particle energies $\left(E_{j e t}^{p c l}\right)$. The Jet Energy Scale (JES) function, shown in Equation 4.4, takes into account the energy from additional $p \bar{p}$ interactions, electronic noise, noise from radioactive decays in the uranium $\left(E_{0}\right.$, the offset energy), and the responses of particles such as photon, pions, kaons, (anti-)protons and neutrons $\left(R_{\text {jet }}\left(E_{\text {jet }}^{\text {meas }}, \eta\right)\right.$, the calorimeter response to the hadronic jet), and the shape of the jet $\left(R_{\text {cone }}\left(\mathcal{R}, E_{\text {jet }}^{\text {meas }}, \eta\right)\right.$, the fraction of particle jet energy contained within the cone $)^{2}$

$$
E_{\text {jet }}^{\text {pl }}=\frac{E_{\text {jet }}^{\text {meas }}-E_{0}(\mathcal{R}, \eta, \mathcal{L})}{R_{\text {jet }}\left(E_{\text {jet }}^{\text {meas }}, \eta\right) \times R_{\text {cone }}\left(\mathcal{R}, E_{\text {jet }}^{\text {meas }}, \eta\right)}
$$

At the analysis level, usable jets are selected by applying cuts on the following variables:

CHF The fraction of the jet $p_{T}$ in the coarse hadronic layers. Candidates which have been reconstructed from noisy cells will tend to fail requirements on this variable.

EMF The fraction of the jet $p_{T}$ in the electromagnetic layers. This helps to reject jets formed by electromagnetic particles which haven’t been reconstructed as electrons or photons.

\footnotetext{
${ }^{2}$ Notice that $E_{0}$ and $R_{\text {cone }}$ depend on $\mathcal{R}$ the radius of the jet cone finding algorithm
} 
HotF The ratio of the $p_{T}$ in the cell with the highest value to that of the next highest cell. This helps to reject jets produced by noise in the calorimeter, where a fluctuation from a single cell can have a significant impact on the reconstructed jet energy.

N90 The number of cells containing $90 \%$ of the total jet $p_{T}$. This too rejects jets produced by noise where most of the energy will come from a small subset of the associated cells.

L1Conf The energy of the L1 jet trigger tower compared to that produced by the jet cone algorithm:

$$
\text { L1Conf }=\frac{\sum_{\text {trigger }} E_{T}^{i}}{p_{T}^{\text {jet }}(1-C H F)}
$$

$E_{T}^{i}$ the transverse EM + Hadronic trigger tower energy within $\Delta R<0.5$ of the reconstructed jet. $p_{T}{ }^{j e t}$ is the momentum of the reconstructed jet with respect to the primary vertex.

\subsection{Electrons and Photons}

The Simple Cone Algorithm, used to form jet candidates, is also used to form electron and photon candidates from calorimeter information. These electromagnetic objects typically deposit most of their energy in the $2^{\text {nd }}$ and $3^{\text {rd }}$ EM layers of the calorimeter. In Run I the energy was primarily deposited in the $3^{\text {rd }}$ layer. With the addition of extra material in front of the calorimeter for Run II their energy deposition distribution has shifted, causing a non-negligible change in the amount of energy deposited in the preshower, solenoid, cryostat walls and central tracker.

The reconstruction begins by forming initial clusters of calorimeter cells using the Simple Cone algorithm [24]. It finds towers with $E_{T}>0.5 \mathrm{GeV}$ as seeds. All the calorimeter towers within a cone of $\Delta R<0.4$ are added to the initial seed, recalculating the centroid-weighted mean after each addition. Clusters with EM Fraction $\frac{E_{E M}(R<0.2)}{E_{T O T}(R<0.2)}>0.9$ and $p_{T}>1.5 \mathrm{GeV}$ are kept as EM candidates.

For each of these EM candidates, starting with the highest $p_{T}$ tower, the reconstruction algorithm calculates the calorimeter isolation. The $E_{T O T}$ is calculated for the $\mathrm{EM}, \mathrm{FH}$, and $\mathrm{CH}$ cells within an $\eta \times \phi<0.4$ tower cone. If the ratio of $\left(E_{T O T}-E_{\text {core }}\right)$ to $E_{\text {core }}$ (the EM energy of the candidate tower in $\Delta R<0.2$ ) is greater than 0.2 , this indicates that the tower is hadronic in origin and the candidate is rejected.

As with jets, an energy scale is applied to the reconstructed candidate. The scale is set by fitting the raw electron energy in $Z \rightarrow e e$ events as a function of $\phi$ and detector rapidity $\left(\eta_{\text {det }}\right)$ such that their reconstructed average mass equals the $Z$ boson mass [25, 26].

There are a number of quality cuts applied at the analysis level to increase the purity of the electron sample.

ID EM clusters are assigned an ID of 10 if they are not matched to a track. Those which do 
satisfy a loose track match are assigned an ID of \pm 11 where the sign indicates the charge of the track.

Isolation Although an initial cut of 0.2 has already been applied as described above, more stringent cuts, such as 0.15 , can be used at the analysis level.

EMFraction EM showers typically deposit 100\% of their energy before reaching the hadronic layers. The cut on this fraction can be as high as 0.97 at the analysis level, but typically it is kept at 0.9 .

H-Matrix For a set of variables the H-Matrix is defined as the inverse of their covariance matrix. This multi-dimensional parameter can be projected into one dimension as a $\chi^{2}$-like variable. Equation 4.8 details the calculation of this parameter. The $h m x 7(h m x 8)$ H-Matrix uses 7 (8) variables listed in Table 4.1

$$
\begin{aligned}
M_{i j} & =\frac{1}{N} \sum_{n=1}^{N}\left(x_{i}^{n}-\left\langle x_{i}\right\rangle\right)\left(x_{j}^{n}-\left\langle x_{j}\right\rangle\right) \\
H & \equiv M^{-1} \\
\chi^{2} & =\sum_{i j}\left(x_{i}^{k}-\left\langle x_{i}\right\rangle\right) H_{i j}\left(x_{j}^{k}-\left\langle x_{j}\right\rangle\right)
\end{aligned}
$$

\begin{tabular}{|c|c|c|}
\hline "hmx7 & hmx8 & Variable \\
\hline $\mathrm{X}$ & $\mathrm{X}$ & $\begin{array}{l}\text { Shower energy fraction in each of the } 1^{\text {st }}, 2^{\text {nd }}, 3^{\text {rd }} \text { and } 4^{\text {th }} \\
\text { EM layers of the calorimeter }\end{array}$ \\
\hline $\mathrm{X}$ & $\mathrm{X}$ & Cluster size in $r-\phi$ based on the $3^{\text {rd }}$ EM layer of the calorimeter \\
\hline $\mathrm{X}$ & $\mathrm{X}$ & Total shower energy \\
\hline $\mathrm{X}$ & $\mathrm{X}$ & Primary vertex position \\
\hline & $\mathrm{X}$ & Transverse width of the shower \\
\hline
\end{tabular}

Table 4.1: The list of variables which constitute the two H-Matrices used to select electrons at $\mathrm{D} \emptyset$.

Track Match Since photons are not reconstructed in the tracker, they can be rejected by requiring the presence of a track matched to the EM cluster. The match is obtained by performing a spatial fit, as defined in Equation 4.9, with respect to tracks from both the CFT and SMT subdetectors. The $\chi^{2}$ probability of the match must be $P\left(\chi_{\text {spatial }}^{2}\right)>10^{-2}$.

$$
\chi_{\text {spatial }}^{2}=\left(\frac{\Delta \phi}{\sigma \phi}\right)^{2}+\left(\frac{\Delta z}{\sigma z}\right)^{2}+\left(\frac{E_{T} / p_{T}-1}{\sigma E_{T} / p_{T}}\right)^{2}
$$


The first two terms constrain the spatial matching of the track using the difference in $\phi-z$ space and the RMS of each of those variables. The $3^{\text {rd }}$ term places constraints on the ratio of the calorimeter EM cluster and track momentum.

Likelihood The probability that an object be an electron $\left(P_{e}(x)\right)$ or background $\left(P_{b k g}(x)\right)$ is calculated as a function for each of $\mathrm{N}$ variables ( 7 in this case). Assuming that they are uncorrelated, the likelihood that an object is an electron can be evaluated as per Equation 4.10.

$$
\mathcal{P}(\boldsymbol{x})=\frac{\prod P_{e}(x)}{\prod P_{e}(x)+\prod P_{b k g}(x)}
$$

The variables used in the likelihood function, and their bounds, are [27]:

- $0.9<$ EMFraction $<1.0$

- $0<h m x 7<50$

- $E_{T} / p_{T}<3.1$

- $P\left(\chi_{\text {spatial }}^{2}\right)>0$

- $|D C A|$ from $\mathrm{PV}<0.05$ for tracks w/ SMT hits

- Number of tracks in a cone of $R(0.05)<5$

- Total track $p_{T}$ in a cone of $R(0.4)<3.5 \mathrm{GeV}$

If any of the a candidate's values do not satisfy the bounds, the candidate is assigned a likelihood value of -1 .

The specific cuts on each of these quality variables is set at the analysis level.

\subsection{Missing Energy}

The total transverse momentum is expected to be zero. Since neutrinos leave no energy in the detector, their presence is recorded as an imbalance in the $E_{T}$ deposited in the detector.

The first step in measuring the missing $E_{T}\left(\notin_{T}\right)$ is to sum the energies in the calorimeter cells. The $\mathrm{CH}$ layers are not used in their calculation; the noise in their cells has too large a negative impact in the resolution of the $\mathbb{E}_{T}$. The raw $\mathbb{E}_{T}$ can be calculated according to Equations 4.11 - Equation 4.13. 


$$
\begin{aligned}
E_{x, y}^{v i s} & =\sum_{\text {cells }} E_{i}^{x, y} \\
\mathbb{E}_{x}=-E_{x}^{v i s} & , \quad \mathbb{E}_{y}=-E_{y}^{v i s} \\
\mathbb{E}_{T}{ }^{C A L} & =\sqrt{\left(\mathbb{E}_{x}\right)^{2}+\left(\mathbb{E}_{y}\right)^{2}}
\end{aligned}
$$

As we have already seen, not all particles deposit their energies in the calorimeter equally. The resolution of the $\mathbb{E}_{T}$ measurement depends on propagating the energies of the corrected reconstructed objects. To avoid double counting the energies, the cells associated with the reconstructed object are removed from the sum of visible energies and replaced by the object's energy itself as per Equation 4.14.

$$
E_{x, y}^{c o r r}=E_{x, y}^{v i s}+\sum_{i} E_{x, y}^{r e c o}-\sum_{j} E_{x, y}^{c e l l}
$$

Where the first sum $i$ runs over the energy corrected objects, and the second sum $j$ runs over the cells associated with these objects. $\mathscr{E}_{T}$ is a notoriously mercurial variable since it relies on the proper reconstruction of all other objects in the event and the good behaviour of the individual detector elements. 


\section{Chapter 5}

\section{B-Tagging}

"On a mis pas mal de temps pour inventer la roue, et beaucoup moins pour inventer la roue de secours."

B-Tagging is the processes through which we select jets which are likely to be the product of $b$-quark decays. Since the b-quark has a mass of $\approx 4.2 \mathrm{GeV}$ and an average lifetime of $\approx 1.5 \mathrm{ps}$, it is expected to travel on the order of a few $\mathrm{mm}$ before decaying. The charged product decays of the $b$-quark will have a vertex displaced from the event's primary vertex, which can be used to help "tag" these $b$-jets [28]. A cartoon of a jet produced from the decay of a $b$-quark can been seen in Figure 5.1.

From an algorithmic point of view, three types of reconstructed objects are typically used for the tagging of $b$-jets:

- Charged particle tracks recorded in the CFT and SMT subdetectors

- Primary vertices built from two or more of these charged particle tracks

- Hadron jets which are reconstructed from energy deposits in the calorimeter

The $b$-tagging used in this analysis relies on three algorithms, described below, which are combined in a Neural Network. A fourth tagging algorithm, based on the semi-leptonic decay of the parton, is used to help measure the selection efficiency of the Neural Network output.

\subsection{Taggability and Vertex Confirmed Jets}

Each of the three algorithms relies heavily on tracking information which has been spatially matched to the hadronic jets. The first step in the process of $b$-tagging process is to determine 


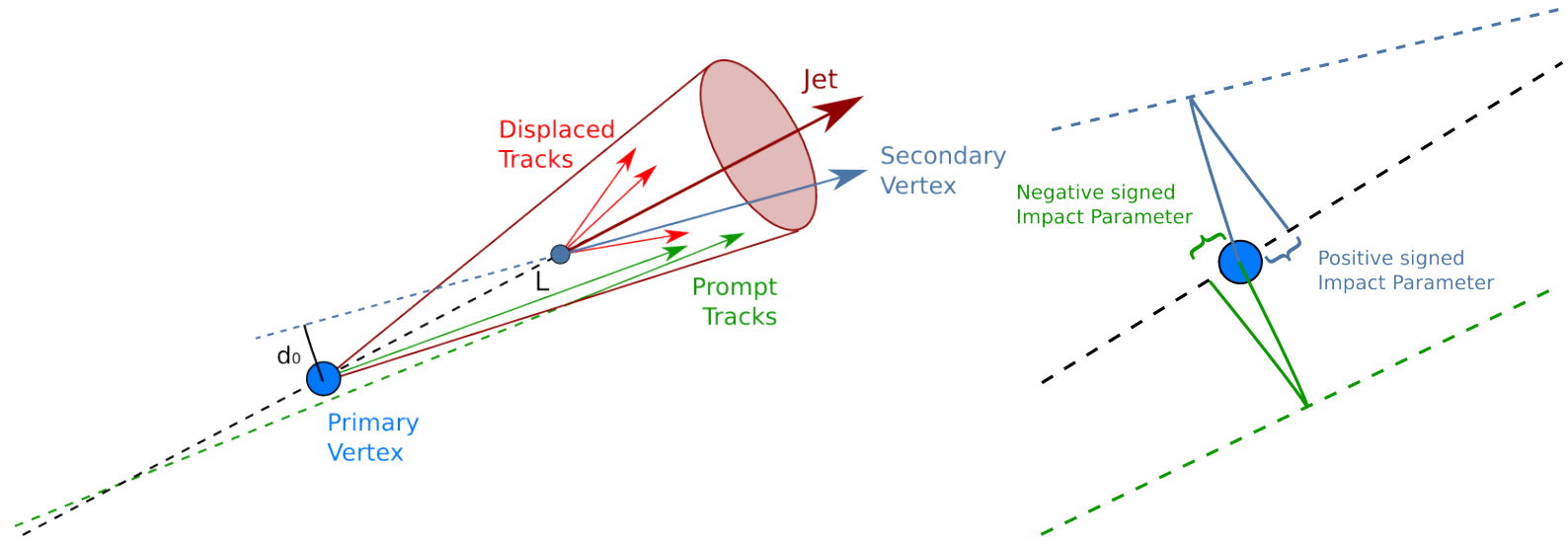

Figure 5.1: A cartoon of a jet produced from the decay of a $b$-quark on the left. A determination of the impact parameter sign on the right.

if the jet itself is taggable. Since the information relies on the displaced decay of the $b$-quark, taggability requires that the hadronic jet is spatially matched to particle tracks in the form of track-jets. To be considered, a track must satisfy the following requirements:

- $\geq 1$ hit in the SMT

- $\mathrm{DCA}($ track, primary vertex $)<2 \mathrm{~mm}$ in the transverse plane

- $\mathrm{DCA}($ track, primary vertex $)<4 \mathrm{~mm}$ in $z$

From this list of tracks, "seeds" are selected, requiring that they have $p_{T}>1 \mathrm{GeV}$. The track clustering is performed using the Snowmass jet algorithm [29] in a cone of $\Delta R<0.5$. The resulting track-jets are associated to the nearest jet within $\Delta R<0.5$.

There is some sample dependence to the track-jet matching requirement. Placing it before running the $b$-tagging algorithms decouples this dependance from the tag rate - which makes it easier to evaluate the tagging performance in data.

In the high luminosity environment of Run IIb, an additional requirement is made prior to taggability to insulate us from electronic noise effects. The jet must be matched to two tracks pointing to the primary vertex.

\subsection{Secondary Vertex Tagger}

The Secondary Vertex Tagger (SVT), calculates a decay length significance (The decay length divided by its uncertainty) for reconstructed secondary vertices. The secondary vertex is determined from the charged particles expected from the displaced $b$-quark hadron's decay.

The reconstruction of the secondary vertex uses the track-jets associated with the calorimeter jet as follows: 
- Select a subset of the tracks in the track-jet, requiring a large impact parameter significance (the impact parameter divided by its uncertainty) in the transverse place :| $\mathcal{S}_{d} \mid>3$

- A Kalman vertex fitting technique [30] is used to reconstruct seed vertices from which the pairs of tracks may have originated.

- The algorithm considers the fit $\chi^{2}$ from adding each of the additional tracks. Only the one resulting in the smallest increase in $\chi^{2}$ is kept. This step is repeated, adding tracks until the $\chi^{2}$ exceeds $\chi_{\max }^{2}=15$.

- Since other processes can produce secondary vertices ( $K^{0}$ decays for example) cuts are made on the direction and displacement of the vertex candidates. The secondary vertex and primary vertices are required to point along the same direction, within $51.5^{\circ}$, and the decay length in the transverse plane must be greater than $2.6 \mathrm{~cm}$.

- The calorimeter jet is then associated with the closest candidate vertex within $\Delta R<0.5$

The $b$-tag is determined based on cuts on the decay length significance of the secondary vertex.

\subsection{The Jet LIfetime Probability Tagger}

The Jet LIfetime Probability Tagger (JLIP) algorithm computes the probability ( $\mathcal{P}_{\text {JLIP }}$ ) that a jet originates from the primary vertex based on the impact parameters of all the tracks associated with the calorimeter jet.

The lifetime probability is calculated from the impact parameter resolution function $\mathcal{R}\left(\mathcal{S}_{d}^{\text {corr }}\right)$, where $\mathcal{S}_{d}^{c o r r}$ is the impact parameter. The impact parameter of a track is assigned a positive value with respect to the jet if it crosses the jet axis in front of the primary vertex. A negative value is assigned if instead it points away from the jet, crossing behind the primary vertex. The sign of the impact parameters is depicted in Figure 5.1. Since the vast majority of light jets originate from the primary vertex, their impact parameter significance distribution is expected to be mirrored around zero. $\mathcal{R}\left(\mathcal{S}_{d}^{\text {corr }}\right)$ is parametrized by four Gaussians using the negative impact parameter significance distribution in data. This value is used to calculate the probability that the track originates from the primary interaction point using Equation 5.1.

$$
\mathcal{P}_{\text {trk }}\left(\mathcal{S}_{d}^{\text {corr }}\right)=\frac{\int_{-\infty}^{-\left|\mathcal{S}_{d}^{\text {corr }}\right|} \mathcal{R}(s) d s}{\int_{-\infty}^{0} \mathcal{R}(s) d s}
$$

The $\mathcal{P}_{\text {JLIP }}$ is calculated from the $N_{\text {trk }}$ tracks with positive decay length significance. 


$$
\begin{array}{r}
\mathcal{P}_{\text {JLIP }}=\mathcal{P}_{\text {JLIP }}+=\Pi \times \sum_{j=0}^{N_{t r k}-1} \frac{\left(-\ln \prod\right)^{j}}{j !} \\
\Pi=\prod_{i=1}^{N_{t r k}} \mathcal{P}_{t r k}\left(\mathcal{S}_{d, i}^{c o r r}\right)
\end{array}
$$

Light flavour jets are expected to produce a uniform $\mathcal{P}_{\text {JLIP }}$ distribution ranging from 0 to 1. Jets from $b$ and $c$ quarks on the other hand will peak at very low values and $b$-jets can therefore be selected by applying cuts on the maximum value of $\mathcal{P}_{\text {JLIP. }}$. The clear advantage of this tagging method is that it depends very little on $\mathrm{MC}$ or the reconstruction of secondary vertices.

\subsection{Counting Signed Impact Parameter Tagger}

The Counting Signed Impact Parameter Tagger (CSIP) also avoids the use of secondary vertices. In this algorithm, the signed impact parameter significance $\left(S_{d}=I P / \sigma(I P)\right)$ is calculated for all good tracks located within a $\Delta R=0.5$ cone around the jet axis. The $b$-tag is then determined based on the number of tracks satisfying cuts on $S_{d}$.

Here, good tracks are expected to be high quality tracks associated with the hard interaction by making the following requirements:

- $\Delta\left(D C A_{z}, P V_{z}\right)<1 \mathrm{~cm}$

- $|D C A|<2 \mathrm{~mm}$

- $p_{T}{ }^{\text {track }}>p_{T}{ }^{\text {min }}$. The value of $p_{T}{ }^{\text {min }}$ depends on the tag point:

- Loose $=1 \mathrm{GeV}$

- Medium $=1.5 \mathrm{GeV}$

- Tight $=2 \mathrm{GeV}$

- Track fit $\chi^{2} / d o f<9$

- Additional cuts on the number of SMT hits associated with the track are determined as follows. Tracks with a $\chi^{2} / d o f<3$ are required to have:

$-\geq 4$ SMT hits and $\geq 14$ CFT hits or

$-\geq 5$ SMT hits and either 0 or at least 11 CFT hits 
The odd combination of requirements on SMT and CFT hits are meant to account for the varrying overlap between the coverage of the two tracking systems.

A jet satisfies the CSIP b-tagging requirement if there are at least two good tracks with $S_{d} / a>3$ or at least three good tracks with $S_{d} / a>2$ where $a$ is a renomalizing parameter picked based on the operating point and the tracks are within $\Delta R<0.5$.

\subsection{The Neural Network Tagger}

Neural Networks (NN) are designed to recognize and exploit the correlations between multiple input variable. The NN Tagger takes as input variables from each of the algorithms described in the three previous sections. It is trained on events, classified as either signal ( $b$-jets) or background (light jets), such that it outputs a continous distribution with signal-like events assigned a value towards 1.0 and background-like events assigned a value towards 0.0.

Six variables are taken from the SVT:

- $\mathcal{S}_{x y}$ : Decay length significance in the transverse plane with respect to the primary vertex.

- $\chi^{2} / \operatorname{dof}$ : The $\chi^{2}$ per degree of freedom of the secondary vertex fit.

- $N_{t r k}$ : Number of tracks used to reconstruct the secondary vertex.

- $m_{v t x}$ : Invariant mass of all the tracks associated with the secondary vertex, assuming each particle is a pion.

- $N_{v t x}$ : Number of secondary vertex associated with the jet

- $\Delta R$ : The distance in $(\eta, \phi)$ space between the jet axis and the difference between the position of the secondary and primary vertices.

Two variables are taken from the JLIP:

- $\mathcal{P}_{\text {JLIP }}$ : The jet lifetime probability output

- $\mathcal{P}_{\text {RedJLIP }}:$ JLIP $\mathcal{P}_{\text {JLIP }}$ recalculated having removed the track with the highest significance.

One variable is taken from the CSIP:

- $N_{C S I P}:$ A combined variable based on the number of tracks with an impact parameter significance greater than the optimized value. Which transforms the inputs into one continuous variable by weighting the individual inputs.

The gains are significant. The NN improves the selection efficiency by $\approx 15-50 \%$ over that of the individual taggers for a fixed purity (See Figure 5.2). The gain in efficiency depends on the $b$-tagging operating point. 


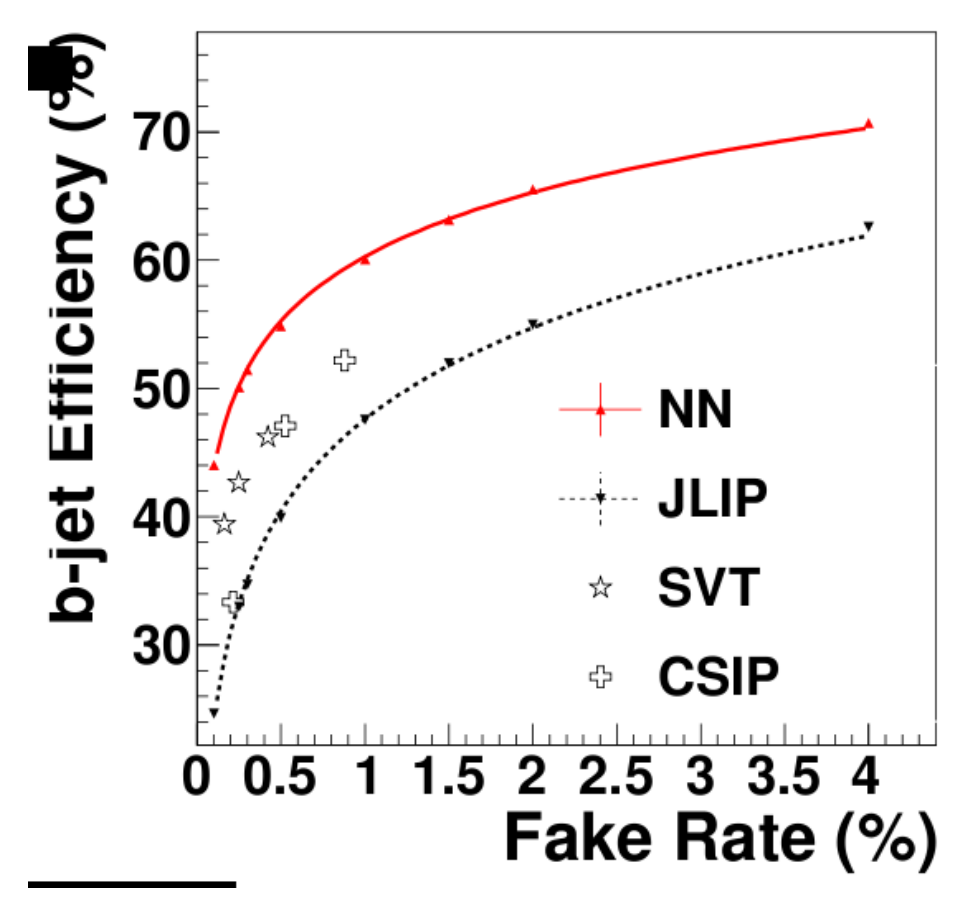

Figure 5.2: Signal efficiency versus background rejection curves for the NN, JLIP, SVT, and CSIP taggers calculated in $Z \rightarrow b \bar{b}$ and $Z \rightarrow q \bar{q}$ MC.

\section{6 $b$ and $c$-jet Tagging Efficiency}

The selection efficiency of the NN tagger is estimated in Data. A sample of $Z \rightarrow b \bar{b}, Z \rightarrow c \bar{c}$, $Z \rightarrow q \bar{q}, \mathrm{QCD}$, and $t \bar{t} \mathrm{MC}$ is used to estimate scale factors to account for differences in Data and $\mathrm{MC}$ between the resolution of the variables and their correlations.

The efficiency measurement is performed using a method called SystemD. SystemD is simply a method of solving linear equations for a system of $D$ unkowns with $D$ equations (where $D$ is 8 in this case) [31].

A heavy flavour jet enriched data sample is selected by making the following three requirements:

- Jet $p_{T}>15 \mathrm{GeV}$

- Jet $|\eta|<2.5$

- Muon with $p_{T}>4 \mathrm{GeV}$ within a distance of $\Delta R<0.5$ of the jet

A second $b$-enriched sample is selected with a muonic jet, as above, and an additional tagged jet.

The samples will contain different mixtures of $b, c$, and light-flavour jets and SystemD will be used to estimate the efficiency of the $b$ and non- $b(\not b)$ samples. Since the fractional content 
of each of these jet flavours depends heavily on the NN output, this process must be performed once for each certified operating point.

The system of equations is as follows:

$$
\begin{aligned}
n & =n_{b}+n_{b^{\prime}} \\
p & =p_{b}+p_{b^{\prime}} \\
n^{S L T} & =\epsilon_{b}^{S L T} n_{b}+\epsilon_{b^{\prime}}^{S L T} n_{b^{\prime}} \\
p^{S L T} & =\epsilon_{b}^{S L T} p_{b}+\epsilon_{b^{\prime}}^{S L T} p_{b^{\prime}} \\
n^{N N} & =\epsilon_{b}^{N N} n_{b}+\epsilon_{b^{\prime}}^{N N} n_{b^{\prime}} \\
p^{N N} & =\epsilon_{b}^{N N} p_{b}+\alpha \epsilon_{b^{\prime}}^{N N} p_{b^{\prime}} \\
n^{S L T, N N} & =\kappa_{b} \epsilon_{b}^{S L T} \epsilon_{b}^{N N} n_{b}+\kappa_{b} \epsilon_{b^{\prime}}^{S L T} \epsilon_{b^{\prime}}^{N N} n_{b^{\prime}} \\
p^{S L T, N N} & =\kappa_{b} \beta \epsilon_{b}^{S L T} \epsilon_{b}^{N N} p_{b}+\kappa_{b} \alpha \epsilon_{b^{\prime}}^{S L T} \epsilon_{b^{\prime}}^{N N} p_{b^{\prime}}
\end{aligned}
$$

where $n$ is the number of jets in the muonic jet sample, $p$ is the number of jets in the $b$-enriched jet sample, and $\epsilon$ is the efficiency of the tagger. The $N N$ super-script refers to the NN tagger. The SLT super-script refer to a method of Soft Lepton Tagging where the $p_{T}$ of the muon projected along the jet axis is required to be greater than $0.5 \mathrm{GeV}$. The $\alpha, \beta, \kappa_{b}$, and $\kappa_{b}$ terms are correlation coefficients. They are:

$\alpha$ the ratio of tagging efficiencies in $\not b$-jets in the two samples.

$\beta$ the ratio of the $b$-tagging efficiencies in the two samples

$\kappa_{b}$ the correlation between the NN and SLT taggers on $b$-flavoured jets

$\kappa_{\not}$ the correlation between the NN and SLT taggers on $\not b$-jets.

Since the SLT method was used in this determination, and all jets in the sample were required to have an assocaited muon, there is some sample bias in the numbers returned by this implementation of SystemD. The ratio of efficiencies measured in an inclusive sample and a semileptonic sample are assumed to be the same in both the Data and MC. The SystemD output is made sample independant by measuring that ratio in $\mathrm{MC}$ and applying a correction to the Data efficiencies.

The same assumption is made about the ratio of of $b$ and $c$-jets. The $c$-jet tagging efficiency used above is also estimated in MC.

\subsection{Tag Rate Function}

To preserve high statistics samples, we do not always wish to directly tag the MC events. Instead, we can apply a weight to the event based on the probability that it does or does 

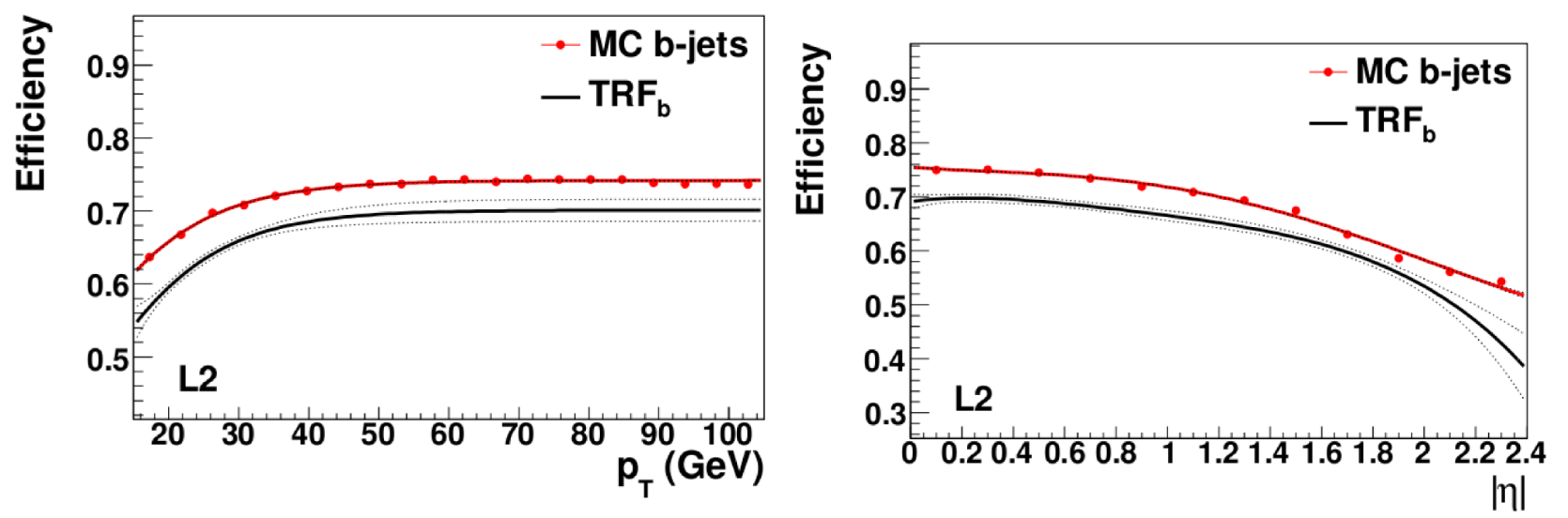

Figure 5.3: The MC inclusive $b$-jet NN tagging efficiency at the L2 operating point (red) and the data TRF (black) as a function of $p_{T}$ (left) and $\eta$ (right).

not satisfy the b-tag requirements. The Tag Rate Functions (TRFs) are the relative difference between the actual and predicted tags in each of the MC samples. The functions are parametrized with respect to $p_{T}$ and $\eta$ for the $b$ and $c$ jets (See Figure 5.3). Just as with the SystemD efficiencies, these TRFs require a scale factor to accurately predict the yields in Data. The TRF functions are as follows:

- $\underline{\mathrm{TRF}_{b}}$ : The efficiency to tag inclusive $b$ jets in data

- $\mathrm{TRF}_{c}$ : The efficiency to tag inclusive $c$ jets in data

- $\underline{\mathrm{SF}_{b}}$ : The factor by which the simulated $b$ and $c$ tagging efficiencies must be scaled to get $\overline{\text { the }}$ data efficiencies. 


\section{Chapter 6}

\section{Limit Setting}

"Sois pas de mauvaise foi. Cette porte est fermée oui ou non?"

After all of the necessary manipulations have been performed on the Data and MC, an analysis is typically boiled down to distribution of histograms in a single variable in which the quantity and shape of the expected signal and backgrounds have been estimated. In this chapter we present an overview of the statistical methods used to interpret this final distribution.

Two main approaches, referred to as Frequentist and Bayesian, have become the de-facto standards in High Energy Physics [32]:

- In the Frequentist approach, the result is interpreted by the frequency at which the observed outcome is likely to occur in a series of hypothetical repeatable experiments.

- In the Bayesian approach, that same result is evaluated to predict where the true value lies based on the best a-priori knowledge of the experimenter.

Either approach can be used to qualify the significance of a result, or in the case where no signal has been found, to set limits on the production rate of the physical process. Since both of the analyses in this thesis use a modified version of the Frequentist method, as implemented in the CoLLIE statistical package [33], we will not be discussing the Bayesian method in further detail.

\subsection{Overview}

The final distribution in question is used to build two hypotheses based on a test statistic calculated in the Data and MC: 
- Signal+Background or TEST $\left(H_{1}\right)$ : Models the Data with MC describing the background processes and the expected signal.

- Background-Only or Null $\left(H_{0}\right)$ : Models the Data with MC which only describes the background. Any knowledge of the signal is expunged in this hypothesis.

In a search, we wish to use a test variable to reject $H_{0}$ in favour of $H_{1}$. The significance level of our result gives us the probability that we have rejected the appropriate hypothesis ( $H_{0}$ in this case). Since some discrepancy between the Data and MC is expected, the $p$-value quantifies the probability that the hypothesis in question would fluctuate to the levels observed in data. It is essentially a test of compatibility between the Data and our hypothesis.

In a counting experiment, the statistical tests would be calculated assuming that the number of events in data have been taken from a Poisson distribution of probable numbers. Since we will be examining continuous distributions, the statistical tests are instead calculated over a range of histogram bins, treated as an array of Poisson distributions. This has the benefit of allowing us to evaluate our test statistic either as a whole, or individually for each channel or sub-processes.

\subsection{The Test Statistic}

\subsubsection{The Log-Likelihood Ratio}

In Collie, the test variable in question is a Log-Likelihood Ratio (LLR), defined in Equation 6.1, which is calculated for the Data, the Null hypothesis, and the TEST hypothesis.

$$
L L R=2 \sum_{i=1}^{N_{\text {channels }}} \sum_{j=1}^{N_{\text {bins }}}\left(s_{i j}-d_{i j} \ln \left(1+\frac{s_{i j}}{b_{i j}}\right)\right)
$$

$i(j)$ ranges over the channels (histogram bins), $d_{i j}$ is the number of data events, $s_{i j}$ is the number of signal events predicted by the $\mathrm{MC}$, and $b_{i j}$ is the number of background events predicted by the MC.

The output of the hypothetical repeatable experiments are expressed in terms of this LLR variable. ColLie generates ensembles of pseudo-experiments for the TEST and NULL hypotheses where the LLR is randomly varied according to the statistical and systematic uncertainties. The interpretation of these distributions is covered in Section 6.3.

\subsubsection{Minimizing Systematics}

Due to the inherent difficulty of measuring systematic uncertainties, the values used often tend to be overestimated. Since we are using histograms, we can use knowledge of the full distribution to produce a more realistic guess of the size of the systematic. This is done by 
minimizing a $\chi^{2}$ variable and treating the size of the systematics as nuisance parameters. The $\chi^{2}$ is defined in Equation 6.2 .

$$
\chi^{2}=2 \sum_{i=1}^{N_{\text {channels }}} \sum_{j=1}^{N_{\mathrm{bins}}}\left(\left(p_{i j}^{\prime}-d_{i j}\right)-d_{i j} \ln \left(\frac{p_{i j}^{\prime}}{d_{i j}}\right)\right)+\sum_{k=1}^{N_{\mathrm{s} y s t}} S_{k}^{2}
$$

The first sum in Equation 6.2 is the Poisson $\chi^{2}$ between the data and our ensemble tests where $p_{i j}^{\prime}$ is the pseudo-data value in the $j^{\text {th }}$ bin of the $i^{\text {th }}$ channel and $d_{i}$ is the data in the $j^{\text {th }}$ bin of the $i^{\text {th }}$ channel. The second sum penalizes the $\chi^{2}$ due to deviations of the systematic correction within its uncertainty, where $S_{k}$ is the number of standard deviations that systematic $k$ is altered. The $\chi^{2}$ is minimized by varying $S_{k}$, which in turn changes the $p_{i j}^{\prime}$ values. This is performed independently for the $H_{0}$ and $H_{1}$ hypotheses, and the LLR is redefined with respect to these fluctuated values. The new LLR is given by Equation 6.3.

$$
L L R^{\prime}=\chi^{2}\left(H_{1}\right)-\chi^{2}\left(H_{0}\right)
$$

\subsection{Confidence Levels and $p$-Values}

The $p$-value is the probability that our hypothesis fluctuates so as to give the values observed in data. From the previously defined LLR variables we can calculate the $p$-value of the results; It is equivalent to the integral of the pseudo-experiment LLR distribution above the value in data.

A confidence level on the other hand gives the guaranteed lower bound on the range of values which contain a measurement's true value for a given significance level. Consider a probability density function (p.d.f) parametrized against the LLR test statistic $(t)$ and some unknown parameter which for us is the signal cross section $(\sigma): f(t \mid \sigma)$. The p.d.f. can be used in Equation 6.4 to give the probability that the signal would yield our observed LLR for any given cross section.

$$
P\left(t_{1}<t<t_{2} \mid \sigma\right)=1-\alpha=\int_{t_{1}}^{t_{2}} f(t \mid \sigma) d t
$$

For a fixed probability, the confidence level is bounded by the range $\left[t_{1}(\sigma, \alpha), t_{2}(\sigma, \alpha)\right]$ of test statistic outputs. In the case where the unknown parameter is physically bounded by 0 , as is the case for our signal cross section, the confidence level and $p$-value have the same interpretation.

Confidence levels can be determined from the LLR integral for the $H_{0}$ and $H_{1}$ hypotheses, which we will call $\mathrm{CL}_{B}$ and $\mathrm{CL}_{S+B}$ respectively. They are the fraction of $H_{0}$ and $H_{1}$ pseudo-experiments with LLR values larger that the one observed in data. 


\subsection{Measuring the Cross Section}

Having covered the concepts of $p$-values, confidence levels, and test statistics, we are now able to state whether we have made a statistically significant observation of the signal. If so, we wish to measure its cross section.

When a signal is observed in the data, the LLR test statistic can be used to determine its cross section in either of two ways:

1. The signal cross section can be left unconstrained in the $\chi^{2}$ minimization of Equation 6.2. This procedure returns a scale factor, along with its error, with respect to the nominal cross section.

2. The signal cross section can be incrementally shifted over a range of values. For each of these, a new set of $H_{1}$ pseudo-experiments are generated. The $1-\mathrm{CL}_{S+B}$ values at each point forms a continuous distribution. The differential of this distribution is a Gaussian centered on the measured cross section.

Although these methods are nominally equivalent, the granularity of the cross section steps in the second method can influence the final result.

If no signal is observed, the confidence levels can also be used to set an upper bound on the cross section of the process. Typically this is done at the $95 \%$ confidence level - by finding the signal cross section for which $1-\alpha=0.95$ in Equation 6.4. When using $\mathrm{CL}_{S+B}$ there is an inherent risk of model biasing in cases where the MC does not agree well with the Data. To protect ourselves from this, we define the exclusion in terms of the ratio of the two confidence levels:

$$
\mathrm{CL}_{S}=\frac{\mathrm{CL}_{S+B}}{\mathrm{CL}_{B}}<\alpha
$$




\section{Chapter 7}

\section{$Z Z \rightarrow \ell \ell \nu \nu$ Analysis}

"Allez, tiens! Je viens d'avoir l'idée d'une experience jamais tentée qui va te distraire pendant que tu prends l'air a la fenêtre."

... plus tard ...

"Dorénavant vous saurez qu'il ne faut jamais charger une arbalète avec un boomerang..."

The Standard Model of Electroweak Interactions makes precise predictions for the couplings of gauge bosons and their cross-sections. Searches for di-boson production, such as $W W, W Z$, or $Z Z$ can provide stringent tests of the predictions. These measurements are of interest because they directly probe the trilinear gauge couplings discussed in Section 1.2.4. The relative rarity of $Z Z$ production makes this channel more statistically limited, as compared to searches for $W W$ and $W Z$. Still, any deviations in this channel could indicate the presence of some new physics, perhaps due to anomalous couplings involving $Z Z Z$ or $Z Z \gamma$. New physics discovered in this sector could possibly lead to a new understanding of Electroweak symmetry breaking.

Although anomalous couplings could provide some insight into new physics, the Higgs mechanism's spontaneous symmetry breaking remains our most likely candidate for explaining the masses of the $W$ and $Z$ bosons. The production cross section times branching ratio of the $Z Z$ process is comparable to that of certain Higgs boson final states (See Figure 7.1). It is important to be able to claim that we have observed and understood all of the SM physics processes which affect searches for the Higgs, especially given the scrutiny that Tevatron Higgs searches now receive.

In this chapter we will describe a search for $Z Z$ production in which one $Z$ decays to either two electrons or two muons and the other $Z$ decays to two neutrinos. The lepton pair will be 


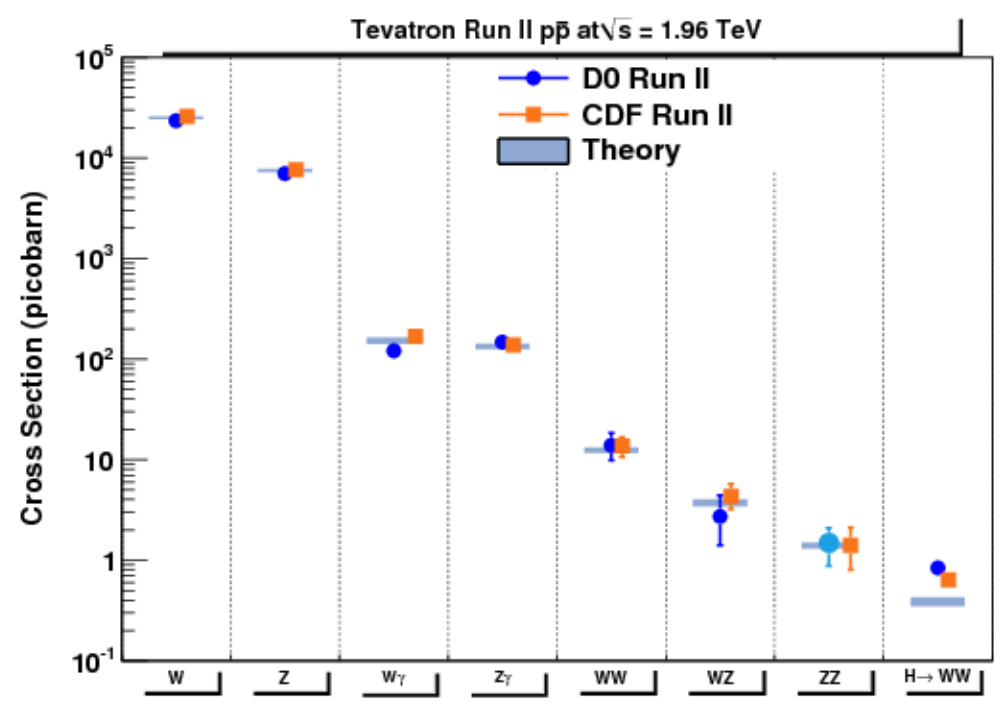

Figure 7.1: With the observation of $Z Z$ production, all of the physics processes involving the production of the Electroweak force-mediating bosons have been observed. This plots shows their cross sections to that of a high-mass Higgs boson.

reconstructed using data provided from the tracking, calorimeter, and muon systems whereas the neutrino pair will appear as an imbalance in the corrected $\mathbb{E}_{T}$.

The cross section times branching ratio of the $Z Z \rightarrow \ell \ell \nu \nu$ process is 6 times larger than for $Z Z \rightarrow \ell \ell \ell^{\prime} \ell^{\prime}$ which has also recently been studied at $\mathrm{D} \varnothing$ [34]. While the $\mathbb{E}_{T}$ is moderately well measured at $\mathrm{D} \varnothing$, with an average resolution of $\approx 8 \mathrm{GeV}$, there are a number of instrumental sources which can result in events with grossly mismeasured values. The dominant background, $Z / \gamma^{*}$ production, occurs at a rate four orders of magnitude larger than our signal. Although $Z / \gamma^{*}$ events are expected to have no significant $\mathbb{E}_{T}$, the relatively small mismeasurement tails can flood the signal region. This is the first time that a measurement of the $Z Z \rightarrow \ell \ell \nu \nu$ process has been attempted at $\mathrm{D} \varnothing$, and a significant portion of this chapter will be dedicated to explaining the analysis techniques we developed to cope with the mismeasured $\mathbb{E}_{T}$.

The backgrounds which contain genuine $\mathbb{E}_{T}$, such as the $W W$, are further separated from the signal through the use of a likelihood variable. It is on this likelihood distribution that we perform our significance calculations. The final result is reported in combination with an independent $Z Z$ search at $\mathrm{D} \emptyset$ where both $Z$ bosons decay to either di-electron or di-muon pairs. 


\subsection{Dataset and Monte Carlo Samples}

\subsubsection{Data}

The data for this analysis were collected between October 2002 and February 2008. The integrated luminosity before data quality requirements is $3.3 \pm 0.2 \mathrm{fb}^{-1}$ in the di-electron and di-muon channels. Data quality information is used to remove runs and luminosity blocks (lbns) marked as "bad" in the calorimeter, muon, CFT, SMT, and luminosity systems. A "bad" status typically indicates that a section of the subdetector in question had been disabled or was in an abnormal state. Events with evidence of noise in the calorimeter are also rejected. This results in a $19.0 \%$ total decrease in integrated luminosity. After data quality cuts, $2.67 \pm 0.11 \mathrm{fb}^{-1}$ are suitable for analysis in the di-electron and di-muon channels.

\subsubsection{Monte Carlo}

Almost all of the signal and background physics processes are modeled by MC produced using Pythia. The lone exception, the $W+$ lp samples, are produced using Alpgen+PythiA. The relative normalization of the PYTHIA samples is set by their cross sections, calculated to Next-to-Leading Order (NLO), before scaling them as a whole to match the $Z$ peak in data after the selection criteria from Section 7.2 have been applied. The matrix method, described in Section 7.3, is used to normalize the $W+$ lp sample to the predicted multijet background contribution. A summary of the MC samples used in this analysis can be found in Table 7.1 and Table 7.2 .

\subsubsection{Additional Monte Carlo Corrections}

The MC corrections common to both analyses in this thesis are described in Section 3.3. Below are additional corrections which were necessary for the $Z Z$ analysis.

\section{Track Efficiency}

There is a reconstruction inefficiency which results in there being a deficit of data events with a small number of tracks and more MC events with a large number of tracks. As a result, the vector sum of total track energy in data is on average $23 \%$ smaller than in MC. This effect is corrected for by performing a two-dimensional random removal of tracks in MC using the reconstruction inefficiency as measured in 20 bins of $\eta$ and 45 bins of $\phi$.

The results of this correction can be seen in Figure 7.2 for Run IIa. 
Table 7.1: p17 signal and background Monte Carlo samples

\begin{tabular}{|c|c|c|c|c|}
\hline Process & Mass Range $[\mathrm{GeV}]$ & 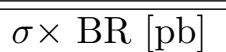 & Generator & Event Count \\
\hline$\overline{\mathrm{Z} / \gamma^{*} \rightarrow \mu \mu}$ & $\overline{15<M<60}$ & 465 & $\overline{\text { PYTHIA }}$ & $2.1 \mathrm{M}$ \\
\hline $\mathrm{Z} / \gamma^{*} \rightarrow \mu \mu$ & $60<M<130$ & 241.6 & Pythia & $3.4 \mathrm{M}$ \\
\hline $\mathrm{Z} / \gamma^{*} \rightarrow \mu \mu$ & $130<M<250$ & 1.96 & PYTHIA & $414 \mathrm{k}$ \\
\hline $\mathrm{Z} / \gamma^{*} \rightarrow e e$ & $15<M<60$ & 465 & РYTHIA & $3.0 \mathrm{M}$ \\
\hline $\mathrm{Z} / \gamma^{*} \rightarrow e e$ & $60<M<130$ & 241.6 & Pythia & $3.7 \mathrm{M}$ \\
\hline $\mathrm{Z} / \gamma^{*} \rightarrow e e$ & $130<M<250$ & 1.96 & Pythia & $407 \mathrm{k}$ \\
\hline $\mathrm{Z} / \gamma^{*} \rightarrow \tau \tau$ & $15<M<60$ & 465 & Pythia & $1.8 \mathrm{M}$ \\
\hline $\mathrm{Z} / \gamma^{*} \rightarrow \tau \tau$ & $60<M<130$ & 241 & PYThIA & $6.9 \mathrm{M}$ \\
\hline $\mathrm{Z} / \gamma^{*} \rightarrow \tau \tau$ & $130<M<250$ & 1.96 & PYTHIA & $409 \mathrm{k}$ \\
\hline$t \bar{t} \rightarrow b b l l \nu \nu$ & & 0.67 & PYTHIA & $325 \mathrm{k}$ \\
\hline$W W \rightarrow l \nu l \nu$ & & 1.29 & PYTHIA & $1.2 \mathrm{M}$ \\
\hline$W Z \rightarrow l \nu l l$ & & 0.12 & PYTHIA & $375 \mathrm{k}$ \\
\hline $\mathrm{ZZ} \rightarrow 1111$ & & 0.014 & PутнiA & $87 \mathrm{k}$ \\
\hline $\mathrm{ZZ} \rightarrow \mathrm{ll} \nu \nu$ & & 0.057 & РутнIA & $253 \mathrm{k}$ \\
\hline$W \gamma \rightarrow e \nu+\gamma(\mathrm{road})$ & & 8.76 & ALPGEN+PYTHIA & $57 \mathrm{k}$ \\
\hline$W \gamma \rightarrow l \nu+\gamma($ prod $)$ & & 5.58 & ALPGEN+PYTHIA & $35 \mathrm{k}$ \\
\hline$W+0 l p \rightarrow l \nu+X$ & & 674 & ALPGEN+PYTHIA & $14 \mathrm{M}$ \\
\hline$W+1 l p \rightarrow l \nu+X$ & & 190 & ALPGEN+PYTHIA & $7 \mathrm{M}$ \\
\hline$W+2 l p \rightarrow l \nu+X$ & & 44 & ALPGEN+PYTHIA & $4 \mathrm{M}$ \\
\hline$W+3 l p \rightarrow l \nu+X$ & & 11 & ALPGEN+PYTHIA & $2 \mathrm{M}$ \\
\hline$W+4 l p \rightarrow l \nu+X$ & & 2.5 & ALPGEN+PYTHIA & $2 \mathrm{M}$ \\
\hline$W+5 l p \rightarrow l \nu+X$ & & 0.85 & ALPGEN+PYTHIA & $1.2 \mathrm{M}$ \\
\hline
\end{tabular}

W+lp MC normalized to the predicted multijet background (See Section 7.3). 
Table 7.2: p20 signal and background Monte Carlo samples

\begin{tabular}{|c|c|c|c|c|}
\hline Process & Mass Range $[\mathrm{GeV}]$ & $\overline{\sigma \sigma \times \mathrm{BR}[\mathrm{pb}]}$ & Generator & Event Count \\
\hline$\overline{\mathrm{Z} / \gamma^{*} \rightarrow \mu \mu}$ & $15<M<60$ & 465 & $\overline{\text { PYTHIA }}$ & $\overline{2.0 \mathrm{M}}$ \\
\hline $\mathrm{Z} / \gamma^{*} \rightarrow \mu \mu$ & $60<M<130$ & 241.6 & Pythia & $2.0 \mathrm{M}$ \\
\hline $\mathrm{Z} / \gamma^{*} \rightarrow \mu \mu$ & $130<M<250$ & 1.96 & РYTHIA & $519 \mathrm{k}$ \\
\hline $\mathrm{Z} / \gamma^{*} \rightarrow e e$ & $15<M<60$ & 465 & Pythia & $1.9 \mathrm{M}$ \\
\hline $\mathrm{Z} / \gamma^{*} \rightarrow e e$ & $60<M<130$ & 241.6 & РYTHIA & $3.8 \mathrm{M}$ \\
\hline $\mathrm{Z} / \gamma^{*} \rightarrow e e$ & $130<M<250$ & 1.96 & РYTHIA & $404 \mathrm{k}$ \\
\hline $\mathrm{Z} / \gamma^{*} \rightarrow \tau \tau$ & $15<M<60$ & 465 & PYTHIA & $2.0 \mathrm{M}$ \\
\hline $\mathrm{Z} / \gamma^{*} \rightarrow \tau \tau$ & $60<M<130$ & 241.6 & РYTHIA & $2.2 \mathrm{M}$ \\
\hline $\mathrm{Z} / \gamma^{*} \rightarrow \tau \tau$ & $130<M<250$ & 1.96 & РYTHIA & $409 \mathrm{k}$ \\
\hline$t \bar{t}$ inclusive & & 6.4 & РYTHIA & $1.5 \mathrm{M}$ \\
\hline$W W \rightarrow l \nu l \nu$ & & 1.29 & РYTHIA & $1.9 \mathrm{M}$ \\
\hline$W Z \rightarrow l \nu l l$ & & 0.12 & РYTHIA & $1.0 \mathrm{M}$ \\
\hline $\mathrm{ZZ} \rightarrow \mathrm{llll}$ & & 0.014 & РYTHIA & $607 \mathrm{k}$ \\
\hline $\mathrm{ZZ} \rightarrow \mathrm{ll} \nu \nu$ & & 0.057 & PYTHIA & $607 \mathrm{k}$ \\
\hline$W+0 l p \rightarrow l \nu+X$ & & 674 & ALPGEN+PYTHIA & $2.3 \mathrm{M}$ \\
\hline$W+1 l p \rightarrow l \nu+X$ & & 190 & ALPGEN+PYTHIA & $2.2 \mathrm{M}$ \\
\hline$W+2 l p \rightarrow l \nu+X$ & & 44 & ALPGEN+PYTHIA & $919 \mathrm{k}$ \\
\hline$W+3 l p \rightarrow l \nu+X$ & & 11 & ALPGEN+PYTHIA & $403 \mathrm{k}$ \\
\hline$W+4 l p \rightarrow l \nu+X$ & & 2.5 & ALPGEN+PYTHIA & $406 \mathrm{k}$ \\
\hline$W+5 l p \rightarrow l \nu+X$ & & 0.85 & ALPGEN+PYTHIA & $238 \mathrm{k}$ \\
\hline
\end{tabular}

$\mathrm{W}+$ lp MC samples are normalized to the predicted multijet background (See Section 7.3). 

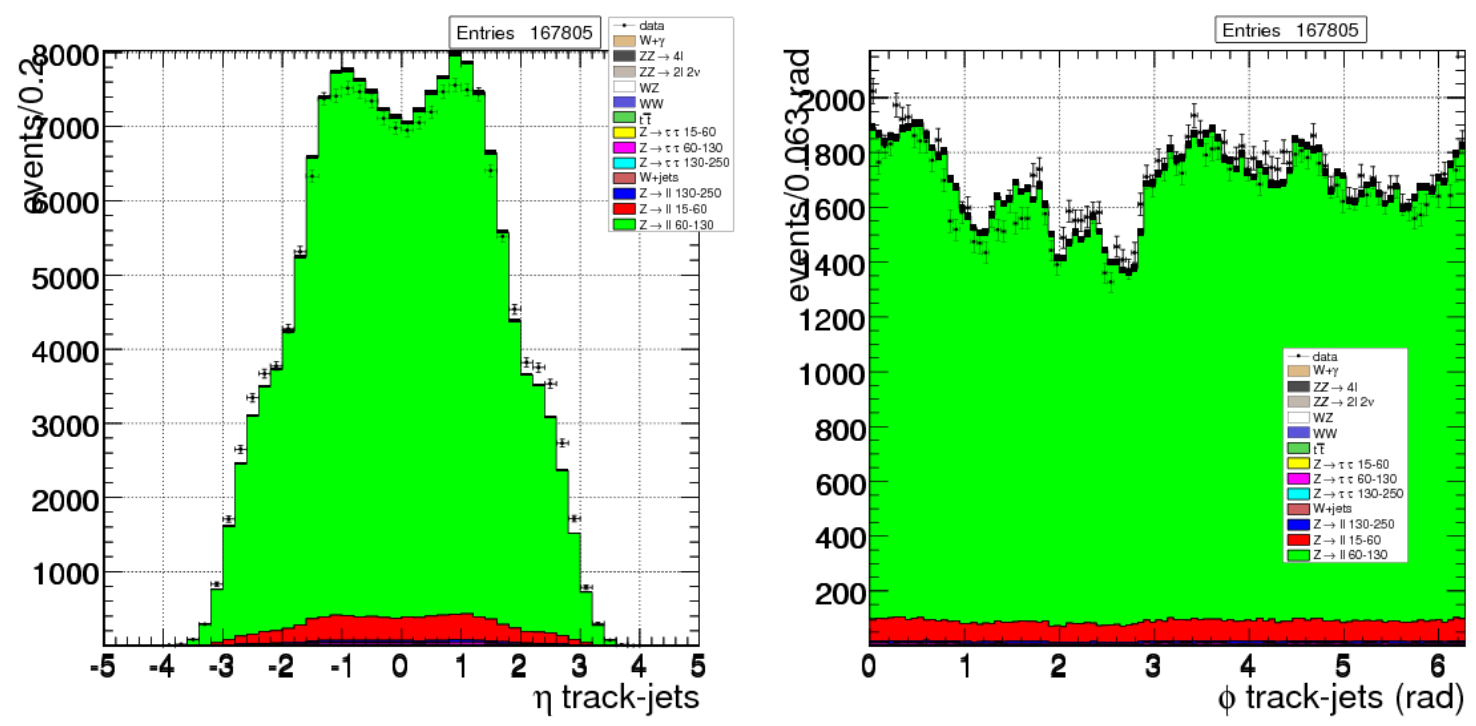

(a) di-electron channel (Run IIa)
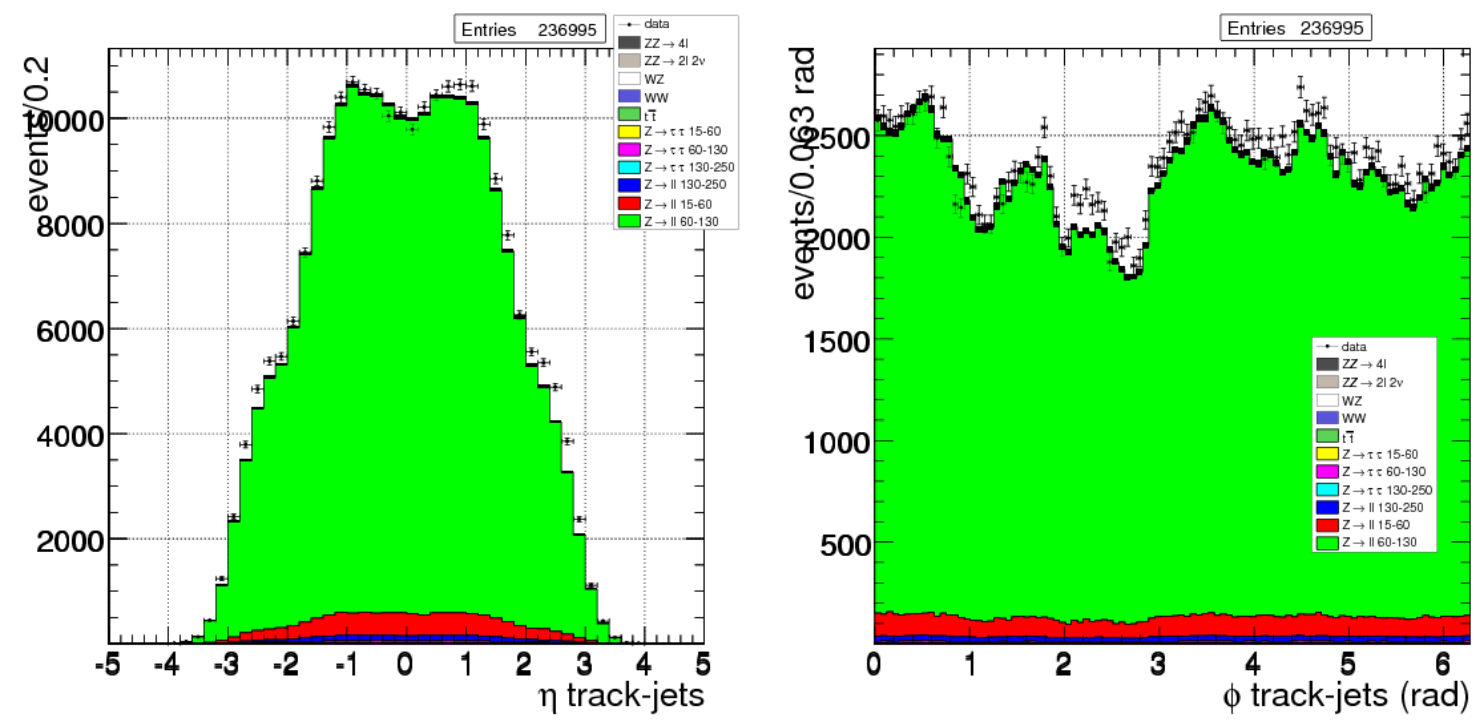

(b) di-muon channel (Run IIa)

Figure 7.2: $\eta$ and $\phi$ distribution of the track-jets after track removal. 


\subsection{Preselection Requirements}

Data are selected using a suite of single electron (single muon) triggers for the di-electron (di-muon) channel. Events must have exactly two leptons with a di-lepton mass in the $70<$ $M_{l l}<110 \mathrm{GeV}$ window and opposite sign charge. We make stringent requirements, vetoing on the presence of any additional leptons. The efficiencies of the various cuts for the signal and background samples in MC are shown in Table 7.3 and Table 7.4. The efficiency of each cut is estimated independantly of the other cuts. The distribution of the di-lepton invariant mass after these requirements are shown in Figure 7.8. At the preselection stage, we make no explicit requirement on the event $\mathbb{E}_{T}$.

\subsubsection{Electron Requirements}

In the di-electron channel, the analysis requires that the events have two EM clusters in the calorimeter which pass cuts on the selection requirements described in Section 4.5. Specifically, these are:

- $p_{T}>15.0 \mathrm{GeV}$

- $I D=10$ or $I D=11$

- Isolation $<0.15$

- EMFraction $>0.9$

- $h m x 7>50$

- Matched to a central track with $p_{T}>5.0 \mathrm{GeV}$

- Likelihood $>0.85$

- $|\eta|<1.1$ or $1.5<|\eta|<2.5$

\subsubsection{Muon Requirements}

Events in the di-muon channel must contain two central-muons reconstructed from scintillator and wire hits as described in Section 4.3. They are required to satisfy the following criteria:

- $p_{T}>15.0 \mathrm{GeV}$

- Loose muon quality

- Matched to a track with at least 1 SMT hit and $\chi^{2} / d o f<4.0$ and $D C A<0.02 \mathrm{~cm}$

- ScaledCalorimeterHalo $<0.1$

- ScaledTrackHalo < 0.1 


\subsubsection{Jet Requirements}

A small number of jets are expected in the events from Initial State (ISR) and Final State (FSR) Radiation. Apart from these jets, and the $Z$-candidate leptons, $Z Z$ events are expected to have very little calorimeter activity. We veto events in which there are more than two jets which satisfy the following requirements, based on the discussion in Section 4.4:

- Cone algorithm with $\Delta R<0.5$

- JES corrected

- $\Delta R($ jet,$\ell$ from $Z)>0.3$

- $E_{T}>15 \mathrm{GeV}$

The low energy threshold, together with the fact that no quality requirements are imposed on the selected jets gives efficient rejection of any registered activity which may generate a fake imbalance in the calorimeter energy. The number of jets above the threshold is shown in Figure 7.3 for the data and MC.

The jets used for this veto are corrected for the JES, and the Jet Shifting Smearing and Reweighting (JSSR) has been applied to the MC samples to reproduce the efficiency and resolution of the detector.

\subsubsection{Additional Leptonic Activity Requirements}

There should be no other leptonic activity in the event apart from that produced by the $Z$ candidates. The presence of any additional charged leptons is a strong indication that the event was produced instead by a source of background, such as $W Z \rightarrow \ell \nu \ell^{\prime+} \ell^{\prime-}$. We have already made the requirement that there be two and only two leptons which satisfy the analysis cuts, we further strengthen this requirement by vetoing on the presence of any additional soft, non-isolated, or poorly reconstructed lepton objects.

\section{Veto Electron Requirements}

Events with one or more extra EM clusters which satisfy the following requirements are discarded:

- $I D=10$ or $I D=11$

- Isolation $<0.2$

- EMFraction $>0.9$

- $E_{T}>5 \mathrm{GeV}$ 

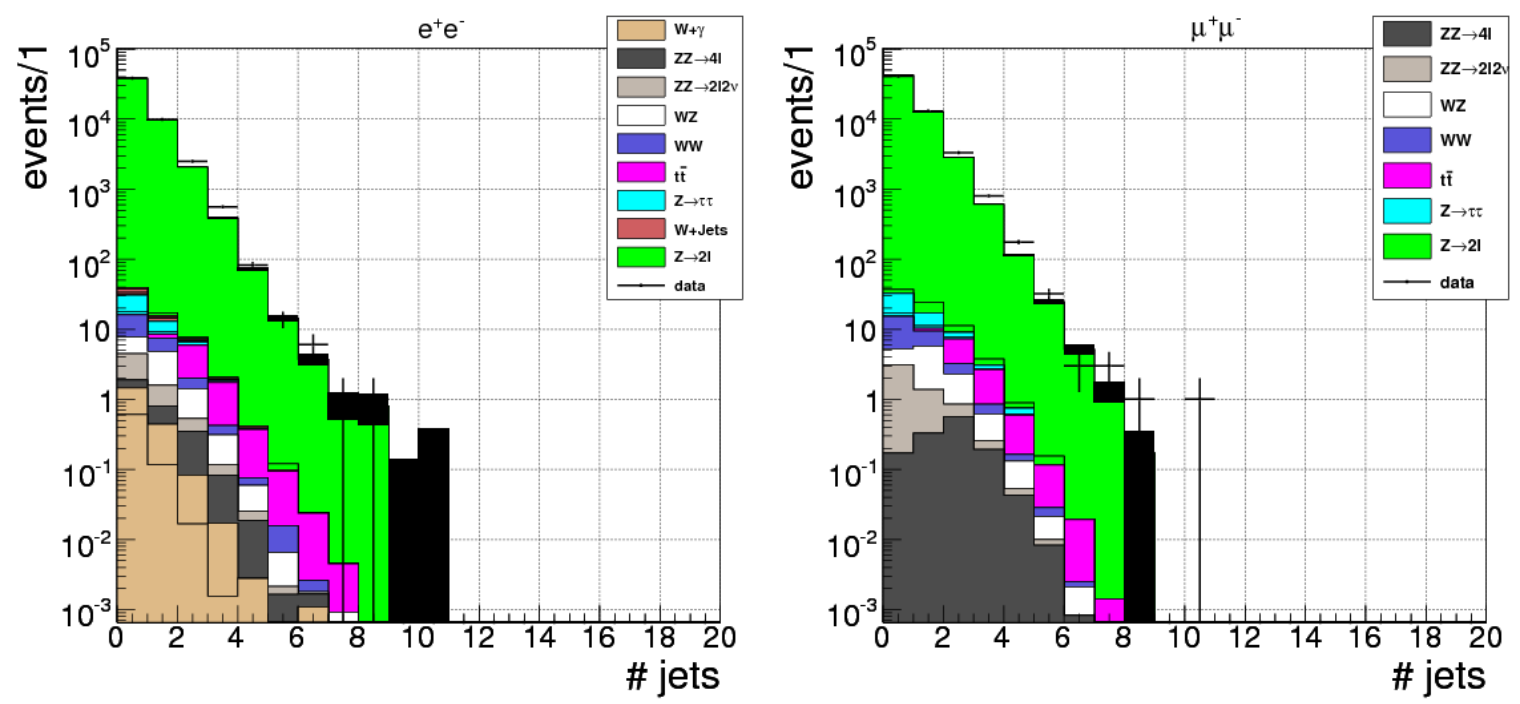

(a) Run IIa
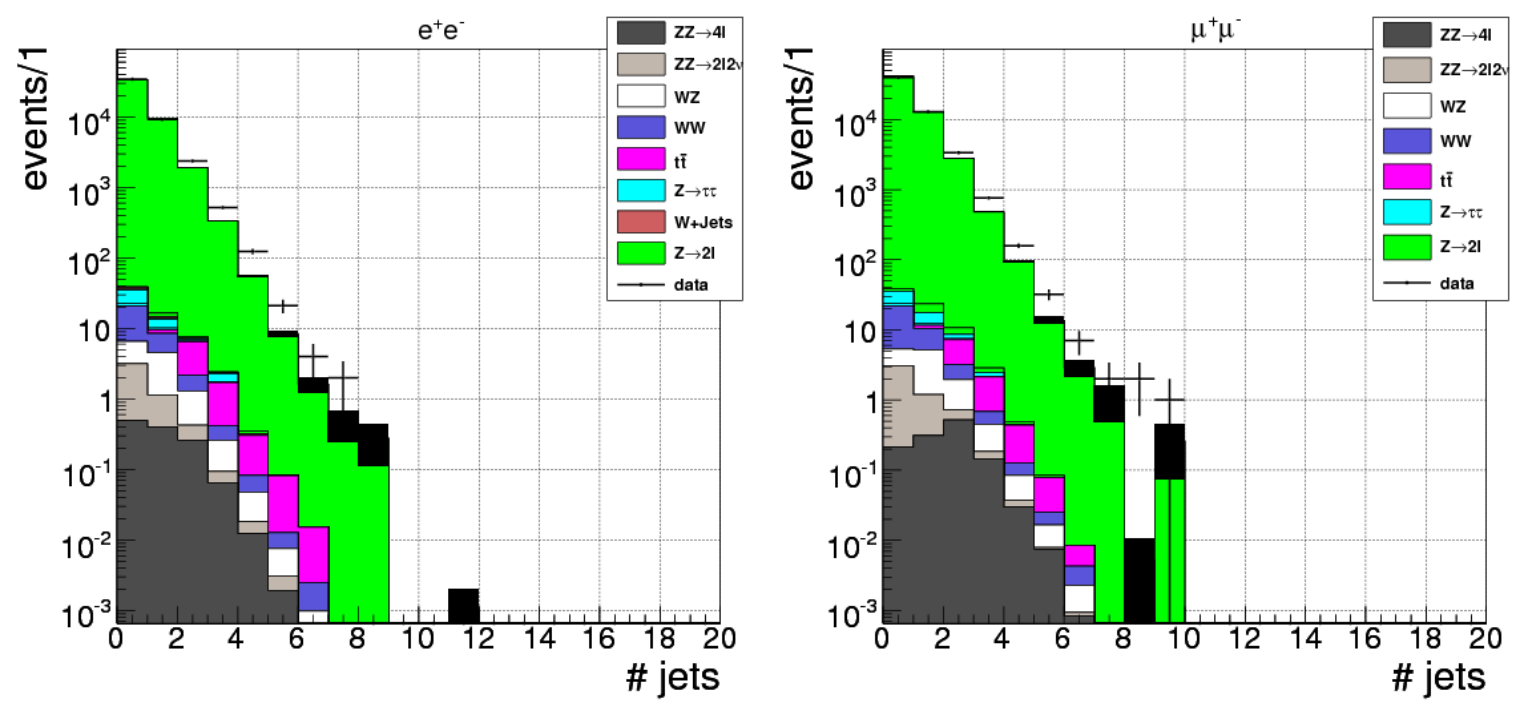

(b) Run IIb

Figure 7.3: Number of jets per event for the di-electron (left) and di-muon (right) final states in Run IIa (a) and Run IIb (b) datasets. Events with more than two jets are rejected.

- $\Delta R($ cluster, $\ell$ from $Z)>0.2$

- $|\eta|<1.1$ or $1.5<|\eta|<2.5$

In addition the cluster must satisfy at least one of the following requirements:

- Matched to a central track with $p_{T}>8 \mathrm{GeV}, \Delta z(\mathrm{PV})<1 \mathrm{~cm}$ and $D C A<0.1 \mathrm{~cm}$ 
- $h m x 7>12$ in $\mathrm{CC}$ or $h m x 8>20$ in $\mathrm{EC}$

The number of clusters satisfying this selection is shown in Figure 7.4 for data and MC.
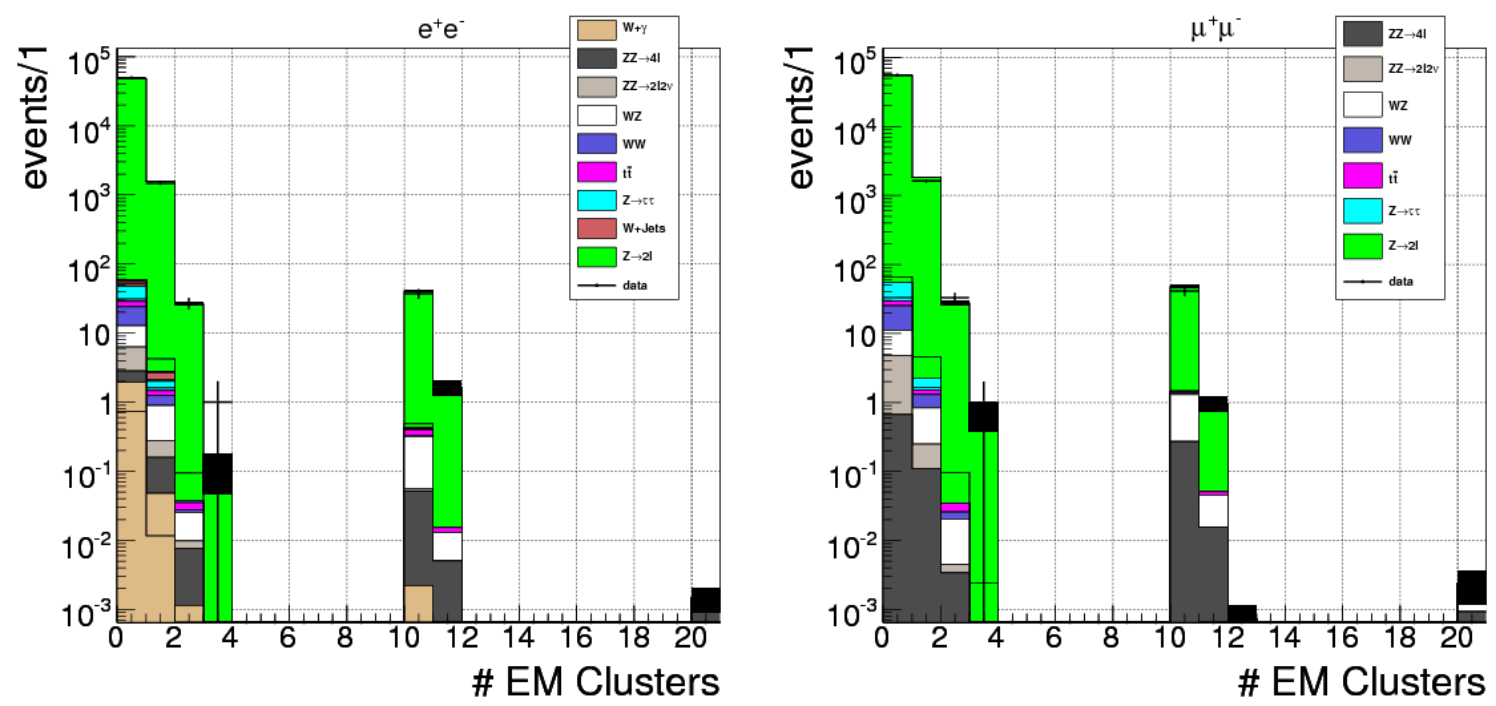

(a) Run IIa
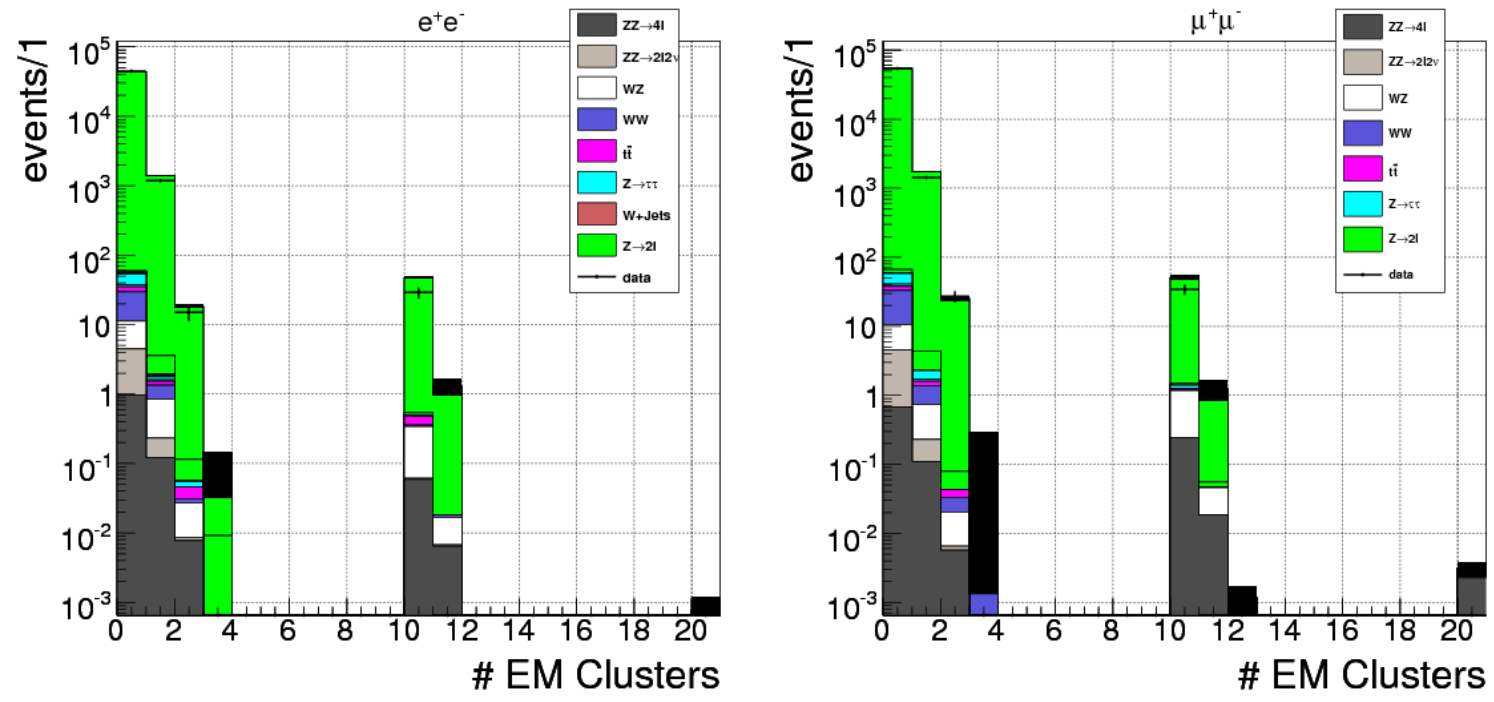

(b) Run IIb

Figure 7.4: Number of EM clusters per event which satisfy the electron veto requirements. Note that the clusters matched to a central track are shifted by 10 to distinguish them in the plot. The plot on the left refers to the di-electron final state while the one on the right refers to the di-muon final state. Run IIa is shown in (a) and Run IIb is shown in (b). 


\section{Veto Muon Requirements}

Events with additional muons other than those produced by the $Z$ decay are rejected. For this purpose the muons are defined as:

- Loose muons associated to a central track

or

- Medium muons

In both cases the muon is required to have a $\Delta R>0.2$ from the $Z$ candidate leptons so as not to double-count the leptons. The number of muons satisfying these requirements is shown in Figure 7.5 .

\section{Veto Tau Requirements}

A veto on the presence of reconstructed multi-prong hadronic tau leptons is applied [35]. The tau objects selection requirements are:

- Tau neural network $N N>0.3$

- Tau type 3

- $p_{T}>5 \mathrm{GeV}$

- $\Delta R(\tau, \ell$ from $Z)>0.2$

- $\Delta z(P V)<1 \mathrm{~cm}$

The number of objects identified as taus according to these criteria is shown in Figure 7.6 for Data and MC.

\section{Isolated Tracks Requirement}

The previous set of vetos are designed to reject anomalous events in which one or more leptons have failed the selection requirements. Similarly, we consider events in which the lepton may have failed the reconstruction requirements entirely. These unreconstructed leptons may still leave hints of their existence in the tracking system, so we veto on the presence of isolated tracks. The number of isolated tracks per event is shown in Figure 7.7. An isolated track must have $p_{T}$ greater than the sum of the surrounding tracks' $E_{T}$. The surrounding tracks are considered in the isolation definition if they satisfy the following criteria:

- $p_{T}>5 \mathrm{GeV}$

- $\Delta R($ track, iso. track $)<1.0$ 

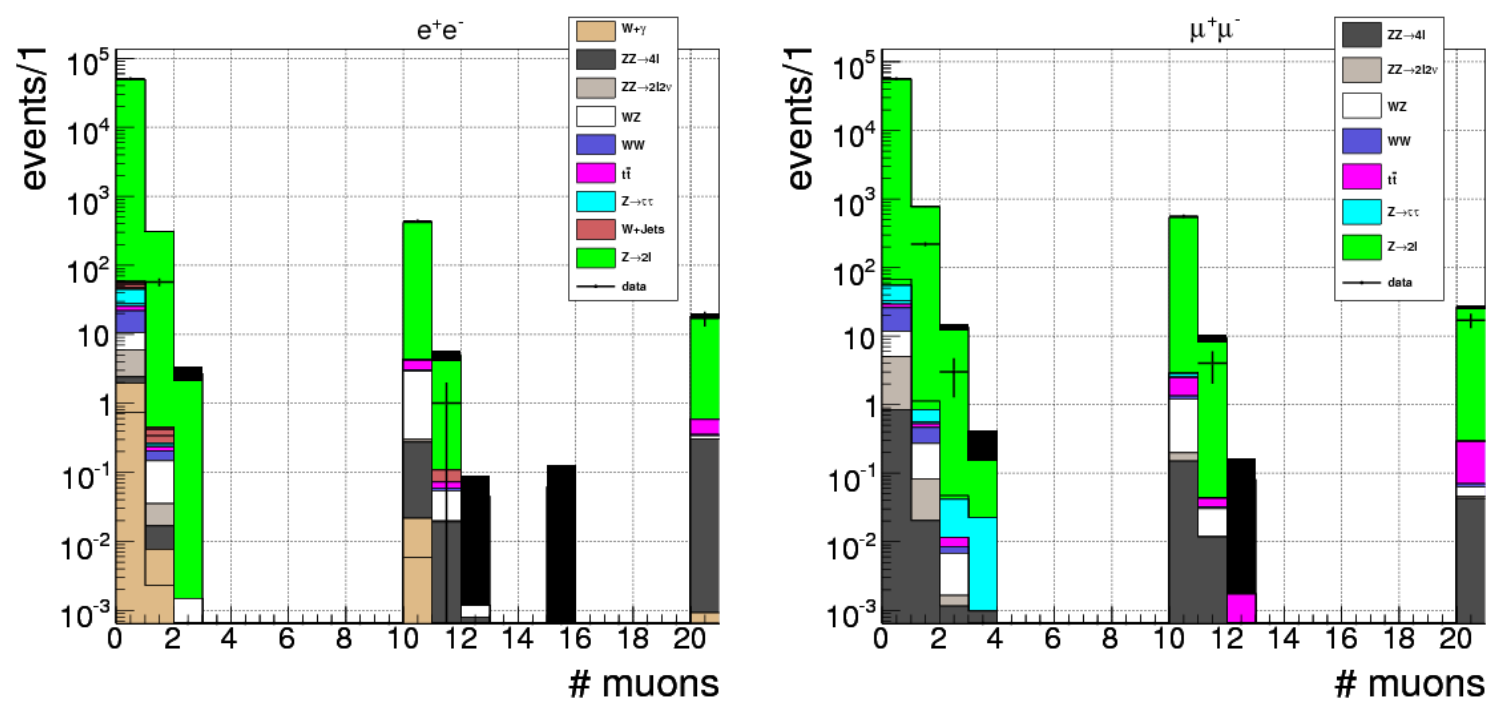

(a) Run IIa
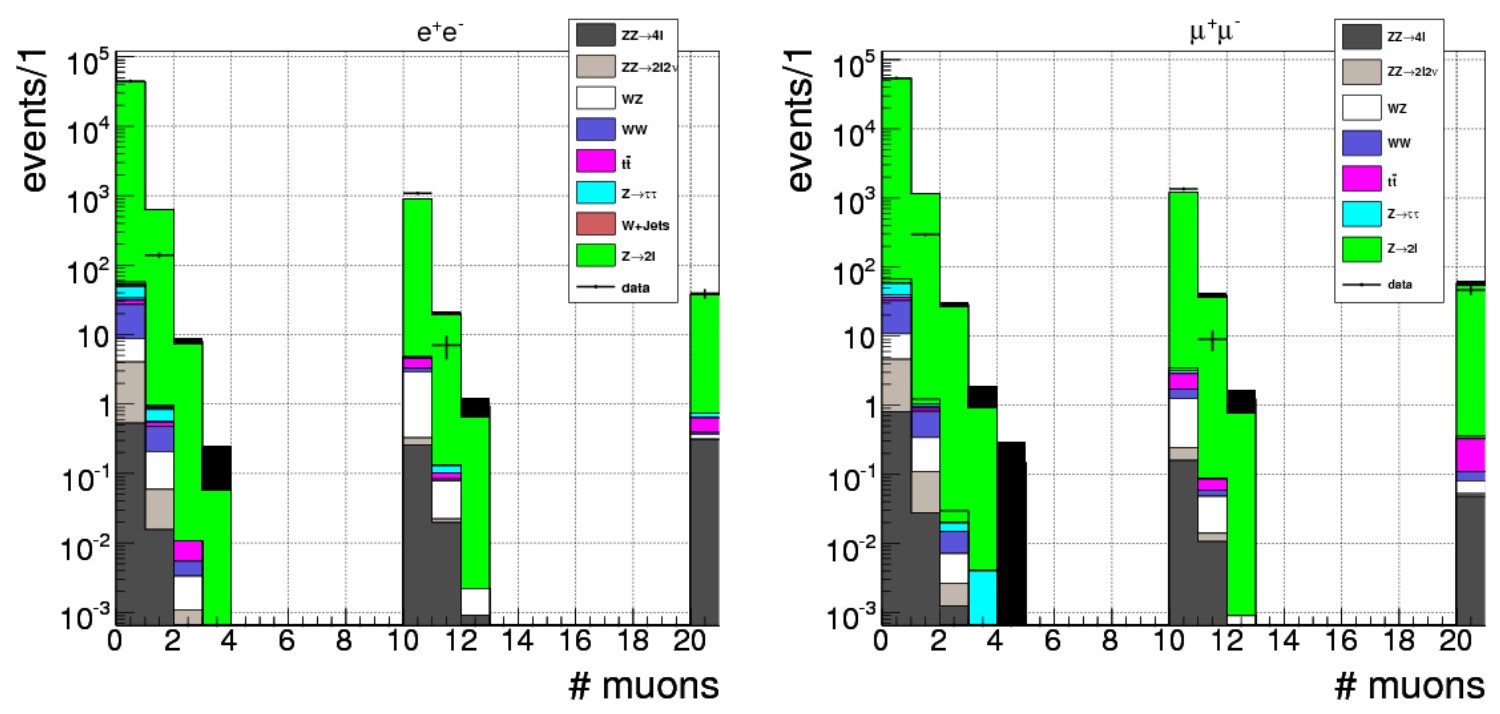

(b) Run IIb

Figure 7.5: Number of poorly reconstructed muons per event not matched to the di-lepton pair from the $Z$. The selection criteria are described in 7.2.4. Note that the muons matched to a central track are shifted by 10 to distinguish them in the plot. Events with one or more muons are rejected. The plot on the left refers to the di-electron final state while the one on the right refers to the di-muon final state. Run IIa is shown in (a) and Run IIb is shown in (b).

- $\Delta z(P V$, track $)<1 \mathrm{~cm}$ 

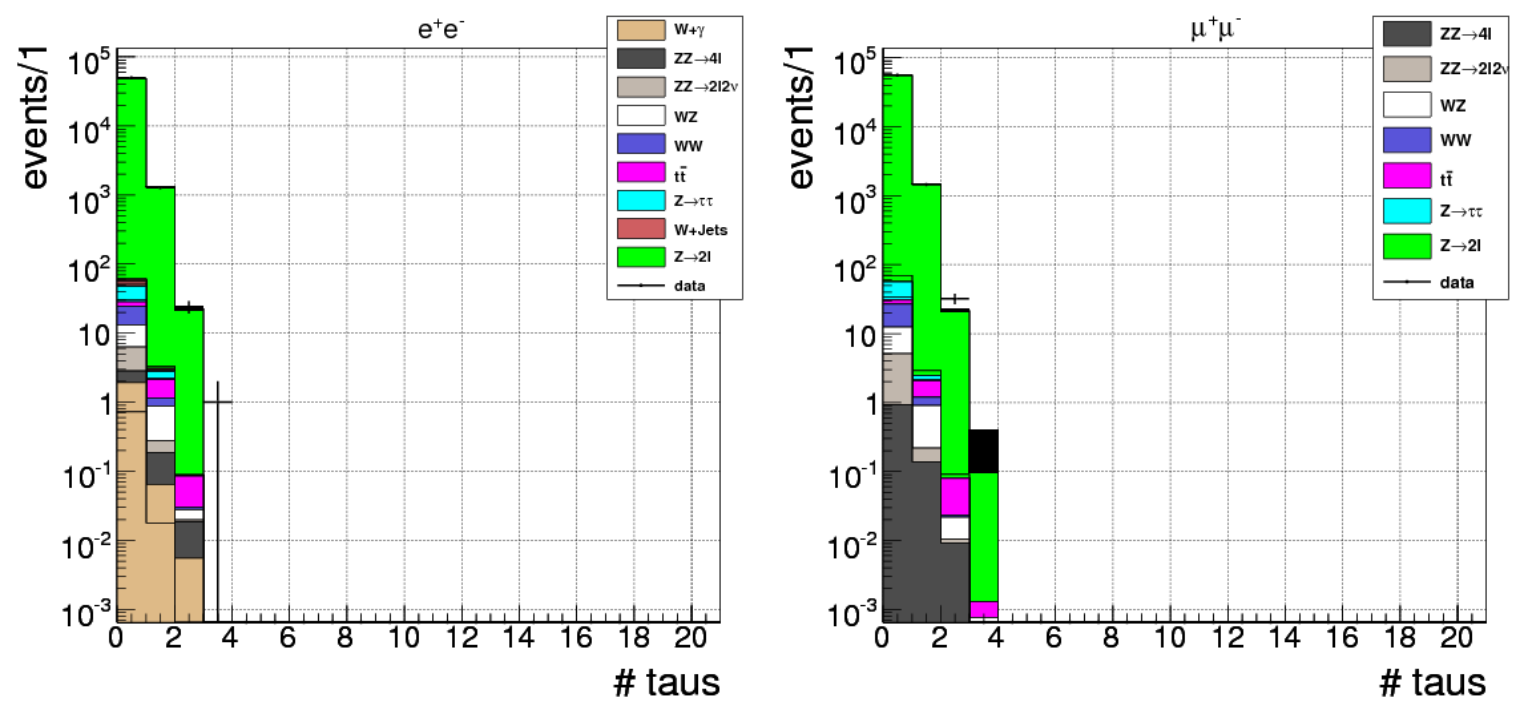

(a) Run IIa
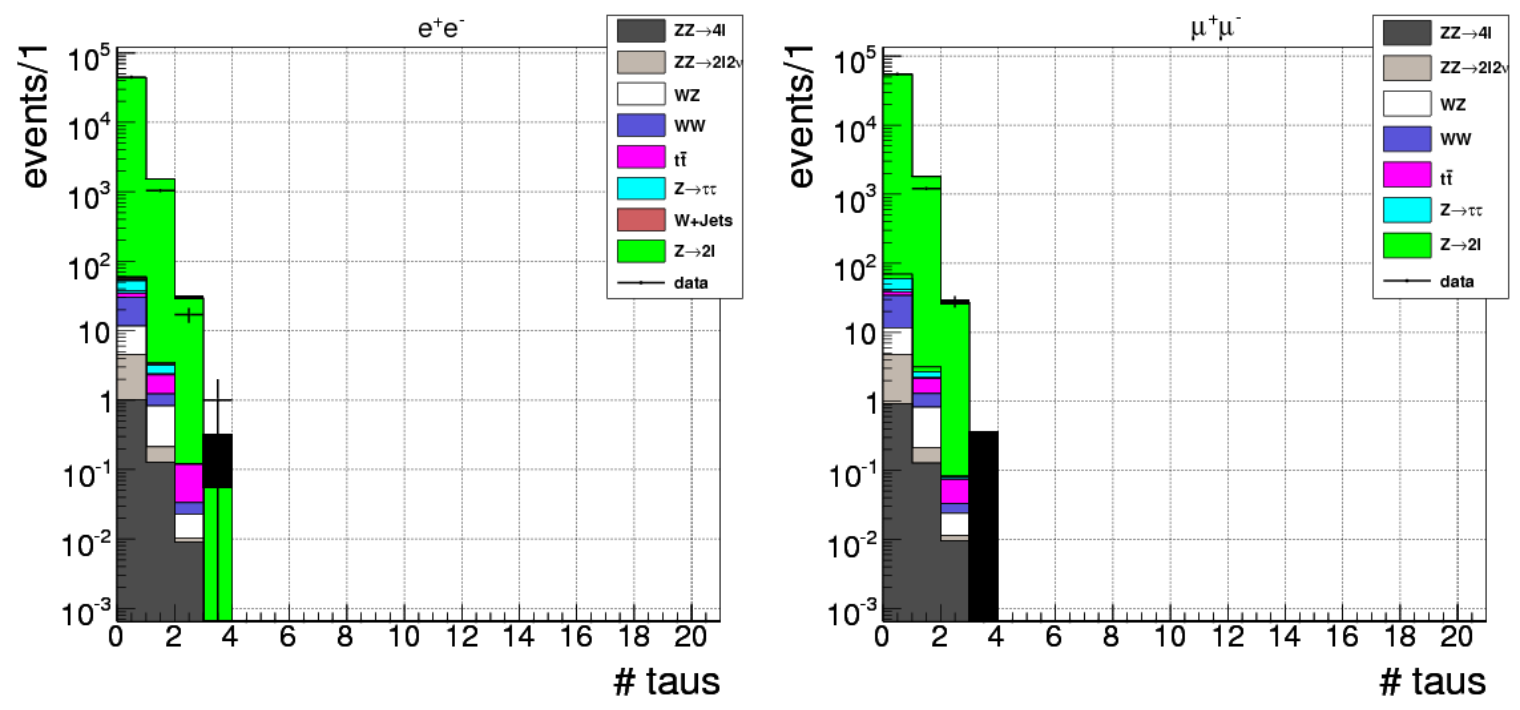

(b) Run IIb

Figure 7.6: Number of reconstructed multi-prong hadronic tau leptons per event. The selection criteria are described in 7.2.4. Events with one or more taus are rejected. The plot on the left refers to the di-electron final state while the one on the right refers to the di-muon final state. Run IIa is shown in (a) and Run IIb is shown in (b).

- $\chi^{2}(P V)<25$

- $E_{T}($ cone $) / p_{T}($ track $)<1$ 
- At least 1 SMT hit or $D C A \leq 0.1 \mathrm{~cm}$

As a demonstration of its efficiency at vetoing additional leptons, a large fraction of the events rejected by the three lepton vetoes also fail the isolated track veto. For this reason, the isolated track veto efficiency dominates the total veto efficiencies listed in Table 7.3 and Table 7.4.

Table 7.3: Efficiencies (\%) of the veto on extra activity for Data and $\mathrm{MC}$ for the di-electron final state. The efficiencies refer to the number of events surviving each exclusive cut.

\begin{tabular}{|lcccccc|}
\hline \hline \multicolumn{7}{c}{ Run IIa } \\
\hline Sample & Jet veto & $e$ veto & $\mu$ veto & $\tau$ veto & iso. track veto & Total \\
\hline Data & $98.71 \pm 0.6$ & $96.85 \pm 0.6$ & $98.93 \pm 0.6$ & $97.23 \pm 0.6$ & $87.30 \pm 0.6$ & $82.86 \pm 0.5$ \\
$Z \rightarrow \ell \ell$ & $99.05 \pm 0.2$ & $96.60 \pm 0.2$ & $98.46 \pm 0.2$ & $97.22 \pm 0.2$ & $88.43 \pm 0.2$ & $83.19 \pm 0.2$ \\
$Z Z \rightarrow \ell \ell \ell \ell$ & $92.69 \pm 1.9$ & $82.53 \pm 1.8$ & $46.39 \pm 1.2$ & $86.27 \pm 1.8$ & $8.62 \pm 0.4$ & $5.04 \pm 0.3$ \\
$Z Z \rightarrow \ell \ell \nu \nu$ & $98.82 \pm 1.3$ & $96.44 \pm 1.3$ & $98.55 \pm 1.3$ & $97.37 \pm 1.3$ & $88.92 \pm 1.2$ & $83.30 \pm 1.2$ \\
$W Z$ & $96.93 \pm 1.1$ & $87.47 \pm 1.0$ & $62.79 \pm 0.8$ & $91.34 \pm 1.0$ & $23.77 \pm 0.4$ & $15.71 \pm 0.3$ \\
$W W$ & $98.85 \pm 1.5$ & $96.74 \pm 1.5$ & $98.20 \pm 1.5$ & $97.48 \pm 1.5$ & $88.37 \pm 1.4$ & $83.08 \pm 1.3$ \\
$t \bar{t}$ & $74.02 \pm 1.7$ & $92.93 \pm 2.0$ & $69.84 \pm 1.6$ & $78.52 \pm 1.8$ & $25.97 \pm 0.9$ & $14.19 \pm 0.6$ \\
$Z \rightarrow \tau \tau$ & $99.33 \pm 6.9$ & $97.23 \pm 6.8$ & $99.30 \pm 6.9$ & $96.74 \pm 6.8$ & $89.01 \pm 6.4$ & $85.02 \pm 6.2$ \\
$W+$ Jets & $98.75 \pm 10.4$ & $93.32 \pm 9.8$ & $97.56 \pm 10.3$ & $97.08 \pm 10.3$ & $81.57 \pm 9.2$ & $72.67 \pm 8.3$ \\
$W+\gamma$ & $98.96 \pm 4.8$ & $97.39 \pm 4.8$ & $98.44 \pm 4.8$ & $96.48 \pm 4.8$ & $86.07 \pm 4.3$ & $82.69 \pm 4.2$ \\
\hline \hline & & & & & \\
\hline \hline & & & & & & \\
\hline Sample & Jet veto & $e$ veto & $\mu$ veto & $\tau$ veto & iso. track veto & Total \\
\hline Data & $98.60 \pm 0.5$ & $97.19 \pm 0.5$ & $97.18 \pm 0.5$ & $97.55 \pm 0.5$ & $84.39 \pm 0.5$ & $79.08 \pm 0.5$ \\
$Z \rightarrow \ell \ell$ & $99.13 \pm 0.2$ & $96.71 \pm 0.2$ & $96.47 \pm 0.2$ & $96.50 \pm 0.2$ & $85.34 \pm 0.2$ & $78.82 \pm 0.1$ \\
$Z Z \rightarrow \ell \ell \ell \ell$ & $93.75 \pm 1.3$ & $82.98 \pm 1.2$ & $46.22 \pm 0.8$ & $87.46 \pm 1.3$ & $9.66 \pm 0.3$ & $5.72 \pm 0.2$ \\
$Z Z \rightarrow \ell \ell 2 \nu$ & $99.03 \pm 1.0$ & $96.71 \pm 0.9$ & $96.57 \pm 0.9$ & $97.44 \pm 0.9$ & $87.05 \pm 0.9$ & $80.77 \pm 0.8$ \\
$W Z$ & $97.52 \pm 0.9$ & $88.28 \pm 0.8$ & $61.12 \pm 0.6$ & $91.59 \pm 0.9$ & $22.62 \pm 0.3$ & $14.84 \pm 0.3$ \\
$W W$ & $98.96 \pm 1.1$ & $97.23 \pm 1.1$ & $96.61 \pm 1.1$ & $97.79 \pm 1.1$ & $87.37 \pm 1.0$ & $81.39 \pm 0.9$ \\
$t \bar{t}$ & $77.75 \pm 3.1$ & $92.85 \pm 3.5$ & $68.04 \pm 2.8$ & $76.76 \pm 3.0$ & $22.00 \pm 1.3$ & $12.11 \pm 0.9$ \\
$Z \rightarrow \tau \tau$ & $96.50 \pm 10.2$ & $98.13 \pm 10.4$ & $97.01 \pm 10.3$ & $95.69 \pm 10.1$ & $86.89 \pm 9.4$ & $79.75 \pm 8.8$ \\
$W+$ Jets & $98.56 \pm 10.3$ & $97.24 \pm 10.2$ & $93.57 \pm 9.8$ & $96.80 \pm 10.2$ & $82.16 \pm 9.1$ & $73.30 \pm 8.3$ \\
\hline \hline
\end{tabular}



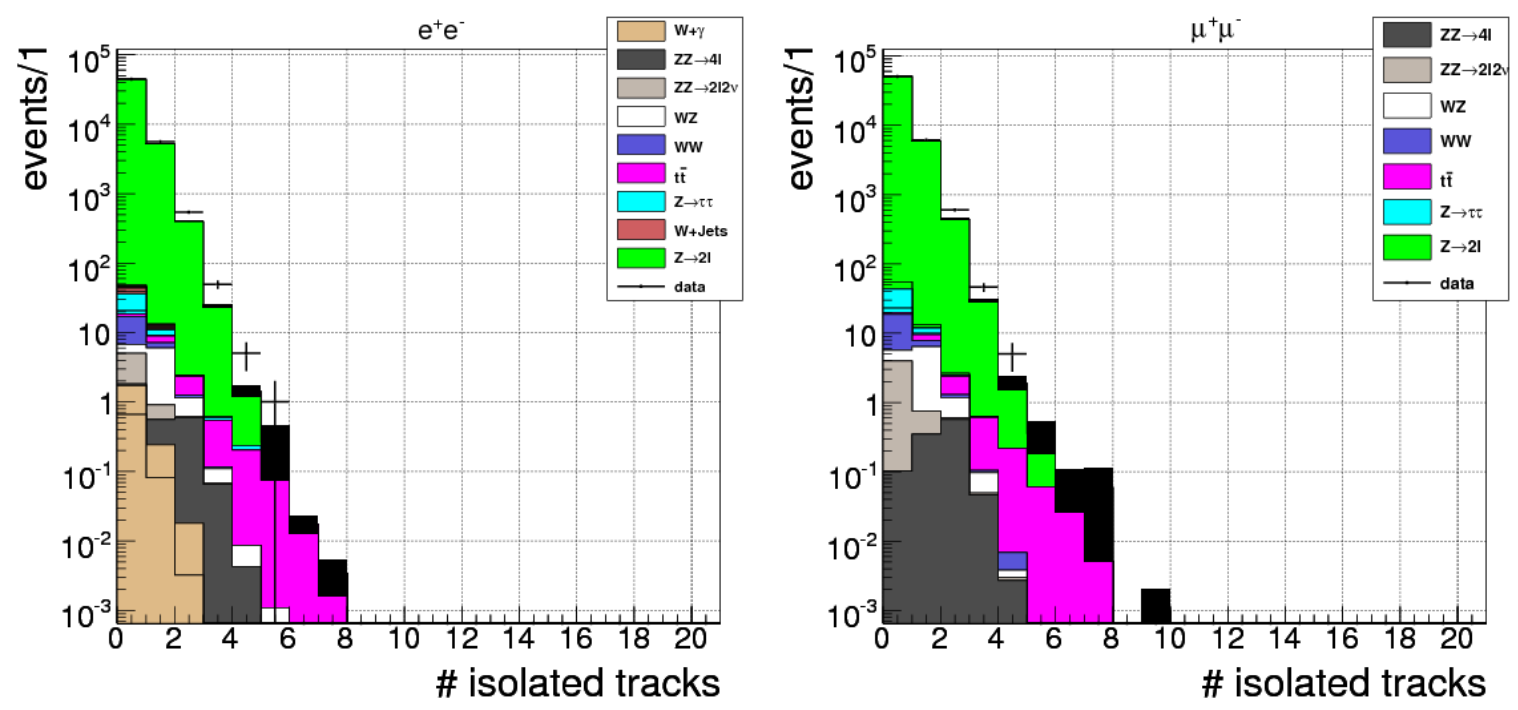

(a) Run IIa
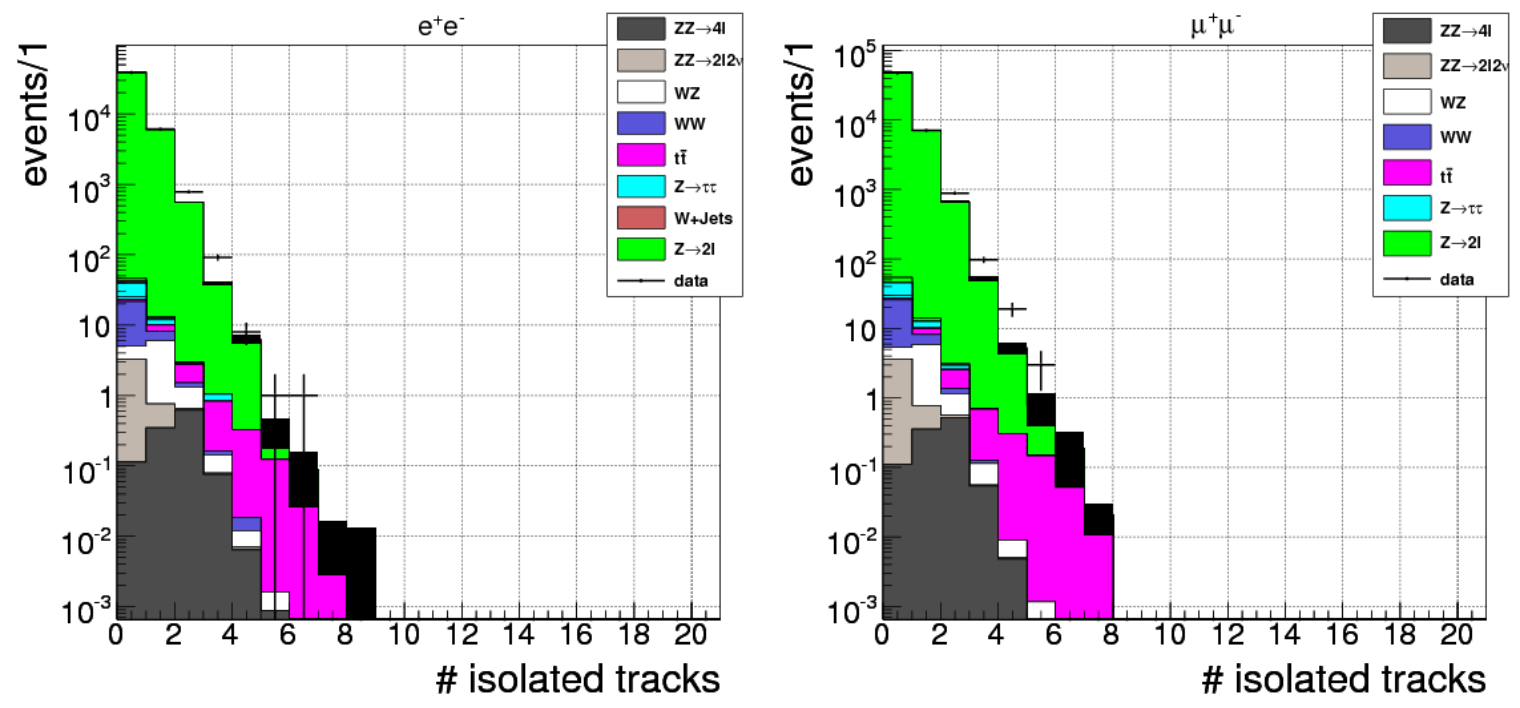

(b) Run IIb

Figure 7.7: Number of veto isolated tracks per event. The plot on the left refers to the di-electron final state while the one on the right refers to the di-muon final state. Run IIa is shown in (a) and Run IIb is shown in (b). 
Table 7.4: Efficiencies (\%) of the veto on extra activity for Data and MC for the di-muon final state. The efficiencies refer to the number of events surviving each exclusive cut.

\begin{tabular}{|lcccccc|}
\hline \hline \multicolumn{7}{c|}{ Run IIa } \\
\hline Sample & Jet veto & $e$ veto & $\mu$ veto & $\tau$ veto & iso. track veto & Total \\
\hline Data & $98.26 \pm 0.6$ & $96.88 \pm 0.6$ & $98.51 \pm 0.6$ & $97.18 \pm 0.6$ & $87.47 \pm 0.5$ & $82.50 \pm 0.5$ \\
$Z \rightarrow \ell \ell$ & $98.71 \pm 0.2$ & $96.61 \pm 0.2$ & $97.59 \pm 0.2$ & $97.28 \pm 0.2$ & $88.53 \pm 0.2$ & $82.44 \pm 0.2$ \\
$Z Z \rightarrow \ell \ell \ell \ell$ & $81.34 \pm 1.6$ & $59.08 \pm 1.3$ & $80.46 \pm 1.6$ & $85.31 \pm 1.7$ & $8.23 \pm 0.4$ & $4.57 \pm 0.3$ \\
$Z Z \rightarrow \ell \ell 2 \nu$ & $98.25 \pm 1.2$ & $96.53 \pm 1.2$ & $97.22 \pm 1.2$ & $97.92 \pm 1.2$ & $89.34 \pm 1.1$ & $82.92 \pm 1.1$ \\
$\mathrm{WZ}$ & $94.58 \pm 1.0$ & $78.56 \pm 0.8$ & $84.56 \pm 0.9$ & $90.79 \pm 0.9$ & $20.75 \pm 0.4$ & $13.49 \pm 0.3$ \\
$\mathrm{WW}$ & $98.19 \pm 1.3$ & $96.52 \pm 1.3$ & $97.53 \pm 1.3$ & $97.74 \pm 1.3$ & $88.65 \pm 1.2$ & $82.44 \pm 1.2$ \\
$t \bar{t}$ & $67.99 \pm 1.5$ & $92.80 \pm 1.9$ & $69.83 \pm 1.5$ & $80.02 \pm 1.7$ & $24.97 \pm 0.8$ & $12.56 \pm 0.5$ \\
$Z \rightarrow \tau \tau$ & $97.95 \pm 5.6$ & $96.96 \pm 5.5$ & $97.26 \pm 5.6$ & $98.43 \pm 5.6$ & $89.93 \pm 5.3$ & $83.49 \pm 5.0$ \\
\hline \hline
\end{tabular}

\begin{tabular}{|lcccccc|}
\hline \hline \multicolumn{7}{c|}{ Run IIb } \\
\hline Sample & Jet veto & $e$ veto & $\mu$ veto & $\tau$ veto & iso. track veto & Total \\
\hline Data & $98.35 \pm 0.5$ & $97.30 \pm 0.5$ & $96.86 \pm 0.5$ & $97.60 \pm 0.5$ & $84.71 \pm 0.5$ & $79.15 \pm 0.4$ \\
$Z \rightarrow \ell \ell$ & $98.97 \pm 0.2$ & $96.73 \pm 0.2$ & $95.51 \pm 0.2$ & $96.65 \pm 0.2$ & $85.71 \pm 0.2$ & $78.33 \pm 0.2$ \\
$Z Z \rightarrow \ell \ell \ell \ell$ & $85.12 \pm 1.2$ & $60.90 \pm 1.0$ & $77.82 \pm 1.1$ & $86.02 \pm 1.2$ & $9.37 \pm 0.3$ & $5.68 \pm 0.2$ \\
$Z Z \rightarrow \ell \ell 2 \nu$ & $98.84 \pm 0.9$ & $96.92 \pm 0.9$ & $95.47 \pm 0.9$ & $97.75 \pm 0.9$ & $88.01 \pm 0.8$ & $80.97 \pm 0.8$ \\
$\mathrm{WZ}$ & $95.79 \pm 0.9$ & $79.66 \pm 0.8$ & $82.50 \pm 0.8$ & $91.35 \pm 0.8$ & $23.06 \pm 0.3$ & $15.56 \pm 0.3$ \\
$\mathrm{WW}$ & $98.78 \pm 1.0$ & $97.00 \pm 1.0$ & $95.59 \pm 1.0$ & $97.81 \pm 1.0$ & $88.07 \pm 0.9$ & $80.81 \pm 0.8$ \\
$t \bar{t}$ & $73.95 \pm 2.8$ & $93.57 \pm 3.4$ & $70.52 \pm 2.8$ & $81.46 \pm 3.0$ & $22.61 \pm 1.3$ & $12.87 \pm 1.0$ \\
$Z \rightarrow \tau \tau$ & $97.84 \pm 9.3$ & $96.11 \pm 9.2$ & $97.97 \pm 9.3$ & $97.03 \pm 9.3$ & $85.32 \pm 8.4$ & $79.84 \pm 8.0$ \\
\hline \hline
\end{tabular}



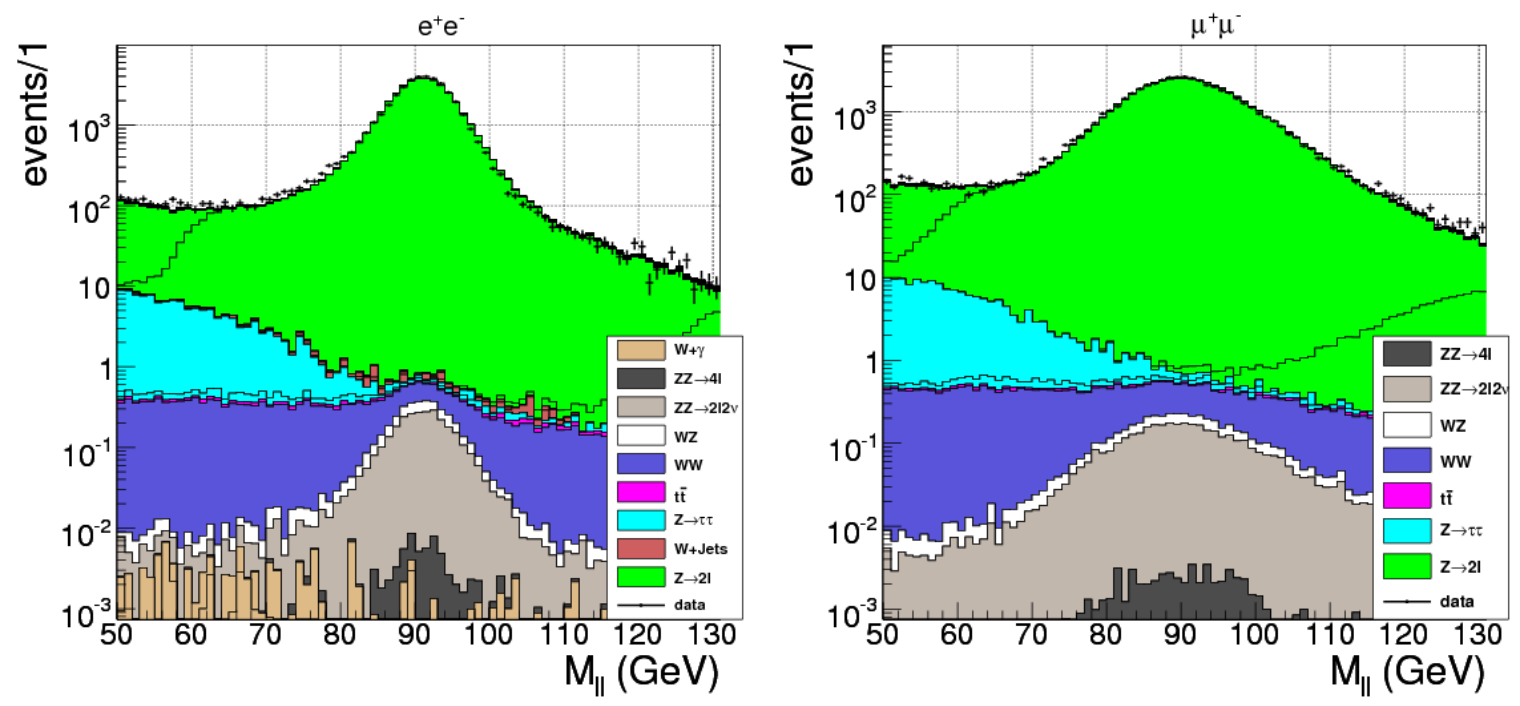

(a) Run IIa
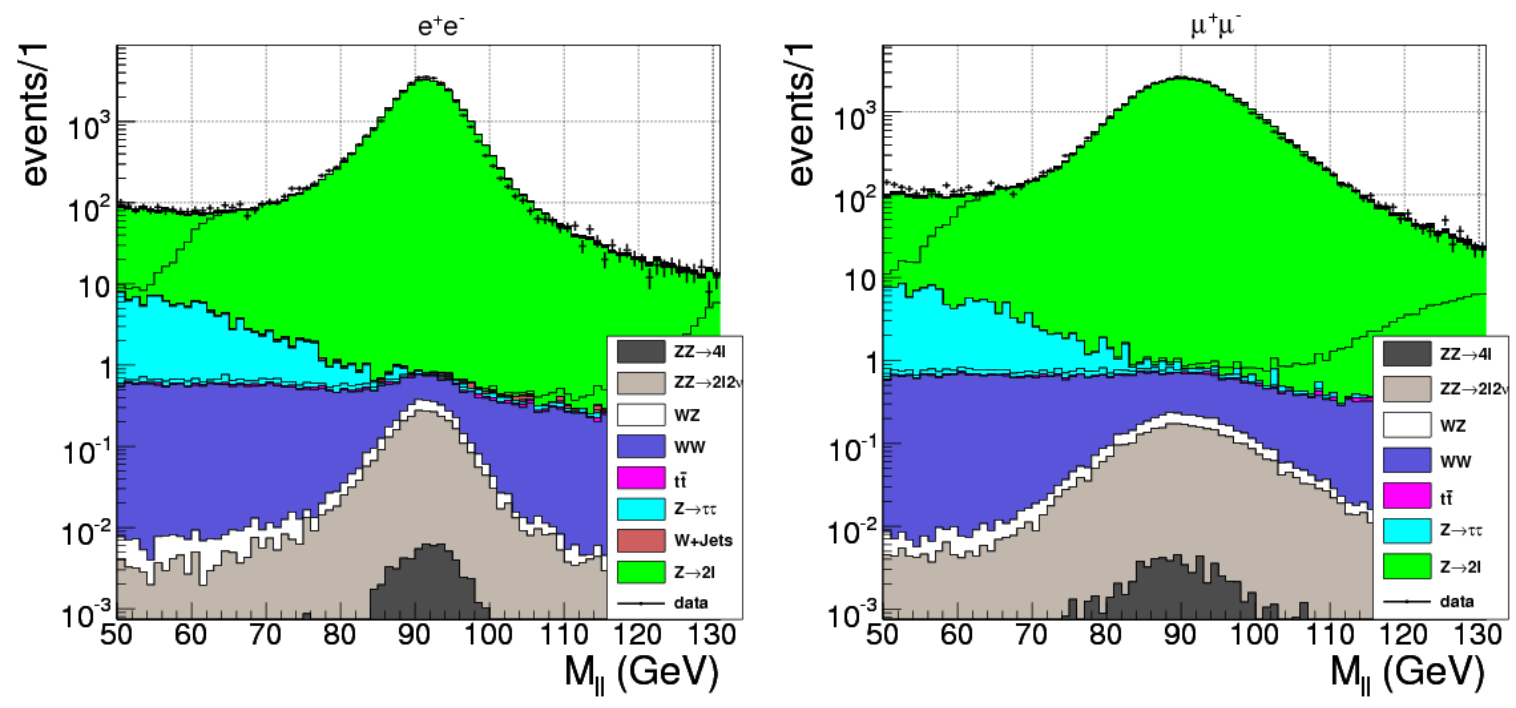

(b) Run IIb

Figure 7.8: Di-lepton invariant mass evaluated after applying the mass cut and the extra activity vetoes for the di-electron (left) and di-muon (right) channels. Run IIa is shown in (a) and Run IIb is shown in (b). 


\subsection{Estimating Instrumental Backgrounds}

The yields of most physics processes in this analysis are estimated using MC, their cross section, branching ratio, and the integrated luminosity of the data sample. Unfortunately, fragmentation is poorly modeled by the generators, on top of which the GEANT simulation of the detector has trouble describing the probability that a jet or photon might mimic the signature of a lepton. The size of the background contribution from $W \gamma$ and $W+$ jets is instead estimated from the data, although the kinematic distributions are still obtained from the $\mathrm{MC}$ samples.

\subsection{1 $W \gamma$ background ${ }^{1}$}

$W \gamma$ events are produced in two ways, although the method in which they are mis-identified as electrons is the same. One $\mathrm{MC}$ sample is used to simulate the final state radiation of a photon in $W$ production diagrams, another MC sample is used to simulate the production of $W \gamma$ pairs through initial state radiation and trilinear vertex diagrams. The relative normalization of these two samples is set by computing their cross sections using the Baur LO Monte Carlo generator [36]. They were generated for events with the following generator level cuts, meant to match the Pyтнia level pre-selection:

- Electron transverse energy: $E_{T}>12 \mathrm{GeV}$

- Angular separation between the electron and photon: $\Delta R(e, \gamma)>0.4$

This sample's contribution is estimated in the data by measuring the probability for a photon to fake an electron with our analysis cuts. The probability that a photon would fake an electron is estimated in $0.74 \mathrm{fb}^{-1}$ of $Z \gamma \rightarrow \ell \ell \gamma$ radiative events [37]. The photons are selected by requiring that a simple cone EM cluster satisfies the following:

- EMFraction $<0.9$

- Isolation $<0.15$

- $E_{T}>15 \mathrm{GeV}$

- $|\eta|<1.1$ or $1.5<|\eta|<2.5$

No explicit cut is made on the $p_{T}$ of the track matched to the EM cluster, the estimates are therefore slightly more on the conservative side. The probability that such an EM object passes the electron likelihood cut of 0.85 is estimated to be $0.012 \pm 0.008$. The number of $W \gamma$ events in the final sample are estimated by selecting the MC with one EM cluster satisfying

\footnotetext{
${ }^{1}$ The work on the $W \gamma$ estimation presented in this section was performed by Gianluca Cerminara
} 
the electron cuts from Section 7.2 and at least one other satisfying the photon cuts listed in the previous paragraph. The events are weighted by applying the 0.012 fake rate. If multiple EM clusters pass the selection requirements, only one candidate is considered. The increased event probability is accounted for by applying the fake rate to both EM clusters.

An explicit requirement is made that the EM cluster be matched to a MC generator level photon. The $W+$ jets sample is estimated in Section 7.3.2 and we want to avoid double counting them.

\subsubsection{W+jets background}

Hadronic jets can also fake leptons in certain cases, and when the jet is produced in association with a $W$ boson the signature becomes very similar to our signal because of the $\mathbb{E}_{T}$ imbalance associated with the $\nu$ in the decay. Moreover, the missing $\mathbb{E}_{T}$ of the event can be artificially increased by instrumental effects related to a jet's inaccurate reconstruction. These considerations, together with the large cross section for the production of $W+j$ ets events make this process the second most important background in the di-electron channel.

As with the $W \gamma$, the MC simulation of the detector cannot be used to estimate the magnitude of this background since the GEANT simulation has not been tuned to reproduce the fake rate of hadronic jets mis-identified as electrons. The $W+$ jets yield in our selected sample is estimated from the data by applying the so called matrix method [38], although the shapes are still modeled by MC.

The matrix method involves solving a system of linear equations with values sampled from two data sets. Events passing all analysis cuts form the tight sample. A second loose sample is defined, removing the likelihood requirement on one of the electrons. The efficiencies of loose background and signal events satisfying the tight sample requirements are $\epsilon_{\text {fake }}$ and $\epsilon_{\text {real }}$ respectively [39].

$$
\begin{aligned}
& N_{l}=N_{b k g}+N_{\text {sig }} \\
& N_{t}=\epsilon_{\text {fake }} N_{b k g}+\epsilon_{\text {real }} N_{\text {sig }}
\end{aligned}
$$

Solving these equations for $N_{s i g}$ and $N_{b k g}$ permits the calculation of the number of signal and background events in the tight sample, along with associated uncertainties. The results are determined in bins of $M_{\ell \ell}$. The size of the bins is selected to maximize granularity while maintaining adequate statistics. The methods used to determine $\epsilon_{\text {fake }}$ and $\epsilon_{\text {sig }}$ are detailed in Appendix A.

The estimated number of $W+$ jets events in the mass window $70<M_{\ell \ell}<110 \mathrm{GeV}$ after all other cuts have been applied is $1.64 \pm 0.5$ for Run IIa and $1.08 \pm 0.3$ for Run IIb in the electron channel. This number has been used to scale the $W+$ jets $\mathrm{MC}$ sample consisting of about 30 million events generated using ALPGEN+PYTHIA. The same calculation performed for the di-muon channel predicts $0.03 \pm 0.05$ events for Run IIa and $-0.02 \pm 0.02$ events for 
Run IIb. Since the number of $W+$ jets predicted in the di-muon channel is consistent with zero, we do not include this sample in the di-muon MC set.

Despite the size of the MC sample used to model the $W+$ jets background, the statistics available at the end of the selection are extremely limited. For this reason the lepton selection has been modified for this background, removing the requirement on the likelihood of the second electron. No distortion in the main quantities is expected from varying the threshold on the electron likelihood cut. 


\subsection{Modified $\mathbb{E}_{T}$ Selection $^{2}$}

There is a sizable fraction of events which are reconstructed with artificially inflated values of $\mathbb{E}_{T}$. This is particularly troublesome since our search is for a signal with genuine $\mathbb{E}_{T}$ but an exceedingly small cross section amidst a background with very little $\mathbb{E}_{T}$. Inclusive $Z$ production in which $\mathbb{E}_{T}$ is misreconstructed can significantly dilutethe signal purity of our sample. This apparent $\mathbb{E}_{T}$ can arise from a mismeasurement of the $p_{T}$ of the individual charged leptons, or a mismeasurement of the hadronic recoil in such events. Achieving any reasonable rejection of the inclusive $Z$ background through cuts on the $\mathbb{E}_{T}$ would require rejecting an unacceptably large fraction of our signal as well (See Figure 7.9).

A new technique is described below for discriminating against events in which the $\mathbb{E}_{T}$ is likely to have been caused by instrumental effects. Although devised with this analysis in mind, it has broader applications in searches for signal with $\mathbb{E}_{T}$ in their final states or conversely, a veto on $\mathbb{E}_{T}$. The crux of the method is to set aside our attempts to improve the resolution of the $\mathscr{E}_{T}$ measurement and instead build an instrumental variable $\left(\mathscr{E}_{T}^{\prime}\right)$ which indicates the minimum feasible $\mathbb{E}_{T}$ in the event. This variable is designed to be robust against sources of mismeasurement. This approach is inspired by one employed in searches at OPAL for the anomalous production of events containing a pair of charged leptons and missing $p_{T}$ [40].

It is difficult to decouple hadronic recoil effects from those due to mismeasurement of the lepton $p_{T}$. To reduce our sensitivity to lepton mismeasurements we project the di-lepton $p_{T}$ onto two axes, one of which is almost insensitive to lepton $p_{T}$ resolution for lepton pairs produced with large opening angles.

As will be detailed later, the decomposition is primarily useful for events in which $\Delta \phi(\ell, \ell)>$ $\pi / 2$, where $\Delta \phi(\ell, \ell)$ is the angle between the two charged leptons in the transverse plane. Three common sources of mismeasurement are identified and used to adjust these projections. The corrections will be covered in the following sections and only a brief overview is provided here.

1. Calorimeter Recoil Activity: We look at data from the calorimeter for any visible additional activity in the event which may account for the apparent $p_{T}$ imbalance. Of particular concern is that the energy resolution of jets is broad. Consider the extreme case where the reconstructed energy of a jet, pointing directly away from the di-lepton pair, is under-measured. This will lead to an apparent $\mathbb{E}_{T}$ in the event which is quite similar to the signature of our signal. The signature might also be faked if any combination of jets have their energies over or under measured.

2. Recoiling Tracks: This correction is designed to account for events in which some recoiling hadronic activity has not been reconstructed into jets. A typical example, might be a jet which has deposited enough of its energy in poorly instrumented regions to fail the reconstruction requirements. The resolution on the measurement of these deposits will

\footnotetext{
${ }^{2}$ The modified $\mathscr{E}_{T}$ variable described in this section was dreamed up by Terry Wyatt
} 

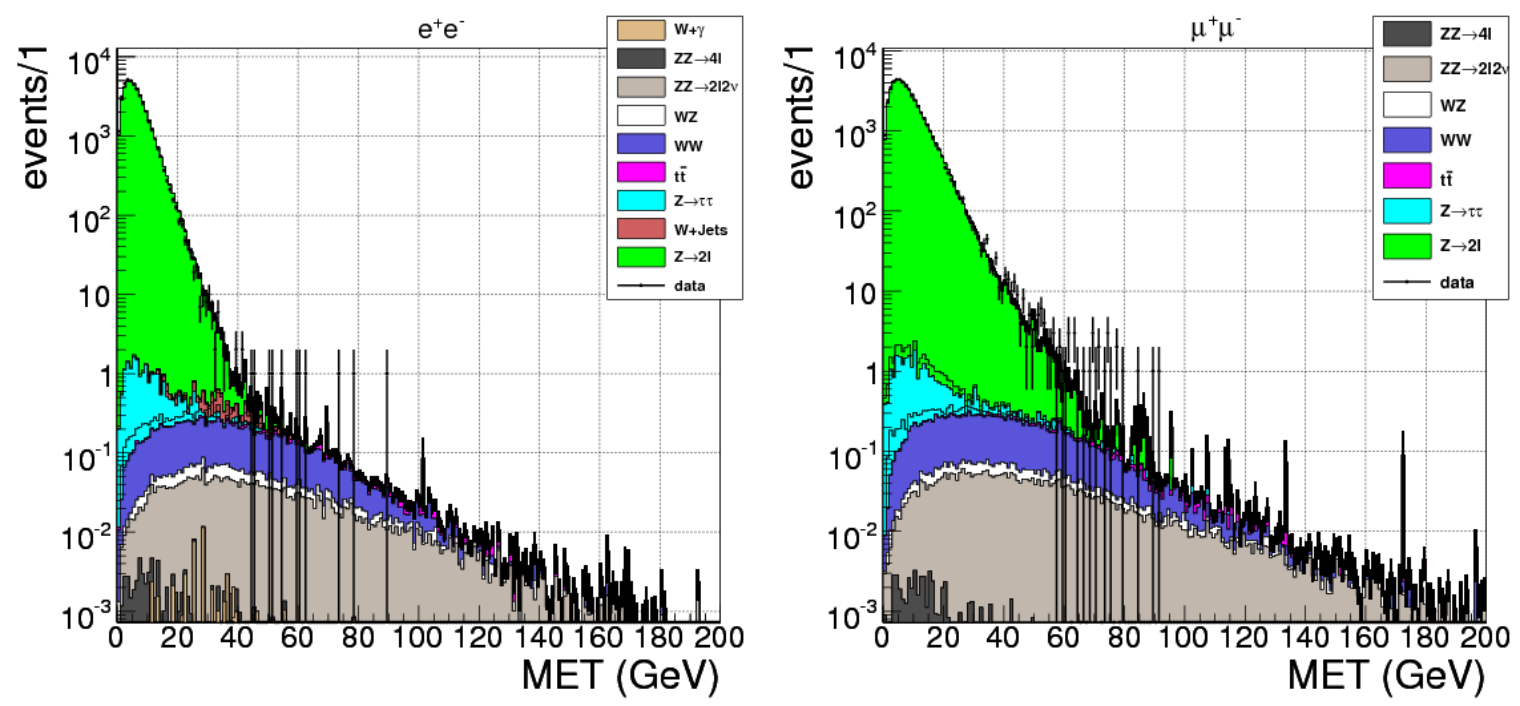

(a) Run IIa
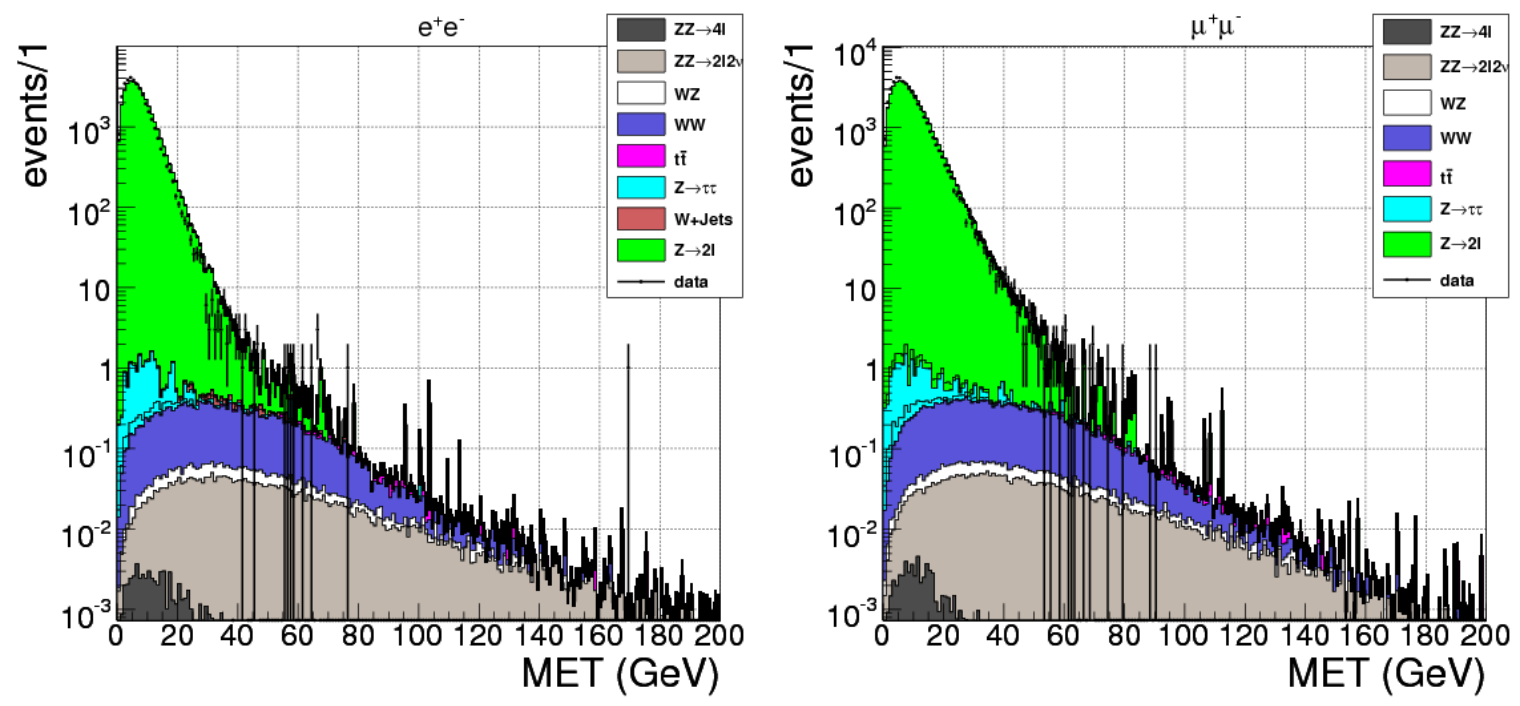

(b) Run IIb

Figure 7.9: $\mathbb{E}_{T}$ of the event evaluated after applying the mass cut and the extra activity vetoes for the di-electron (left) and di-muon (right) channels.

not have benefited from the energy scale corrections applied to jets and electrons, once again producing an apparent $\mathbb{E}_{T}$ where there should be none.

3. Lepton Momentum: The leptons' transverse momenta may also be mismeasured. The uncertainty on the leptons' $p_{T}$ is used to fluctuate the two objects so as to minimize the 
magnitude of the di-lepton $p_{T}$. Remember that our signal is expected to produce $\mathbb{E}_{T}$ equal in magnitude, and pointing directly away, from the di-lepton pair. This correction will reduce the profile of the recoiling $p_{T}$ which must be accounted for.

$\mathscr{E}_{T}^{\prime}$ is formed by a sum in quadrature of the fully corrected components, giving greater weight to the component that is less sensitive to lepton $p_{T}$ mismeasurements. After these manipulations, the resulting variable is a strong indication of the largest probable $\mathbb{E}_{T}$ in the event. It is not, strictly speaking, a measurement of the $\mathbb{E}_{T}$, but rather a $\mathbb{E}_{T}$-based discriminant for rejecting background. 


\subsubsection{Decomposition}

The di-lepton thrust axis (See Figure 7.10 in the transverse plane is defined as:

$$
\boldsymbol{t}=\boldsymbol{p}_{\boldsymbol{T}}^{(1)}-\boldsymbol{p}_{\boldsymbol{T}}^{(2)}
$$

where $\boldsymbol{p}_{\boldsymbol{T}}^{(1)}\left(\boldsymbol{p}_{\boldsymbol{T}}^{(2)}\right)$ is the transverse momentum of the highest (second highest) $p_{T}$ lepton. This is the axis which maximizes the scalar sum of the projection of the $p_{T}$ of the two leptons along the axis. It is used to decompose $p_{T}^{\ell \ell}$ into transverse $\left(a_{t}^{\ell \ell}\right)$ and longitudinal $\left(a_{l}^{\ell \ell}\right)$ components along $\hat{a}_{t}$ (the thrust axis) and $\hat{a}_{l}$ (perpendicular to the thrust axis) respectively.

\section{thrust axis}

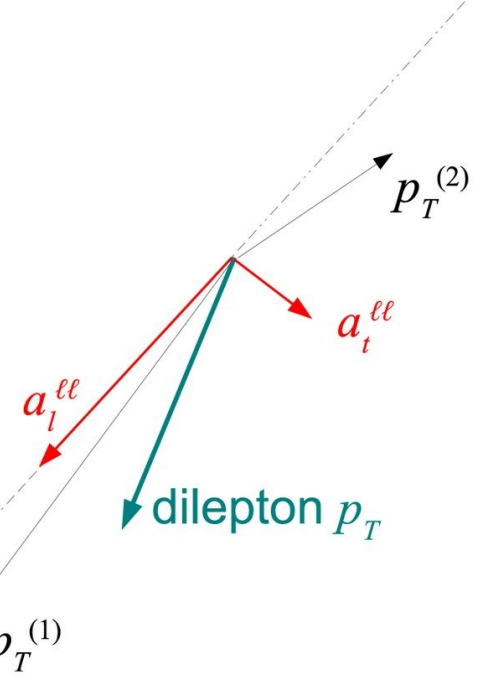

Figure 7.10: The thrust axis of the di-lepton pair is defined as $\boldsymbol{t}=\boldsymbol{p}_{\boldsymbol{T}}^{(1)}-\boldsymbol{p}_{\boldsymbol{T}}^{(2)}$. The $a_{t}^{\ell \ell}$ and $a_{l}^{\ell \ell}$ are the projections of the di-lepton $p_{T}$ along and perpendicular to the thrust axis.

For events with large di-lepton opening angles $(\Delta \phi(\ell, \ell))$, the $a_{t}$ component is much less sensitive to the leptons' $p_{T}$ measurement resolution than $a_{l}$. This property is demonstrated in Figure 7.11 and will be exploited in the final step. 

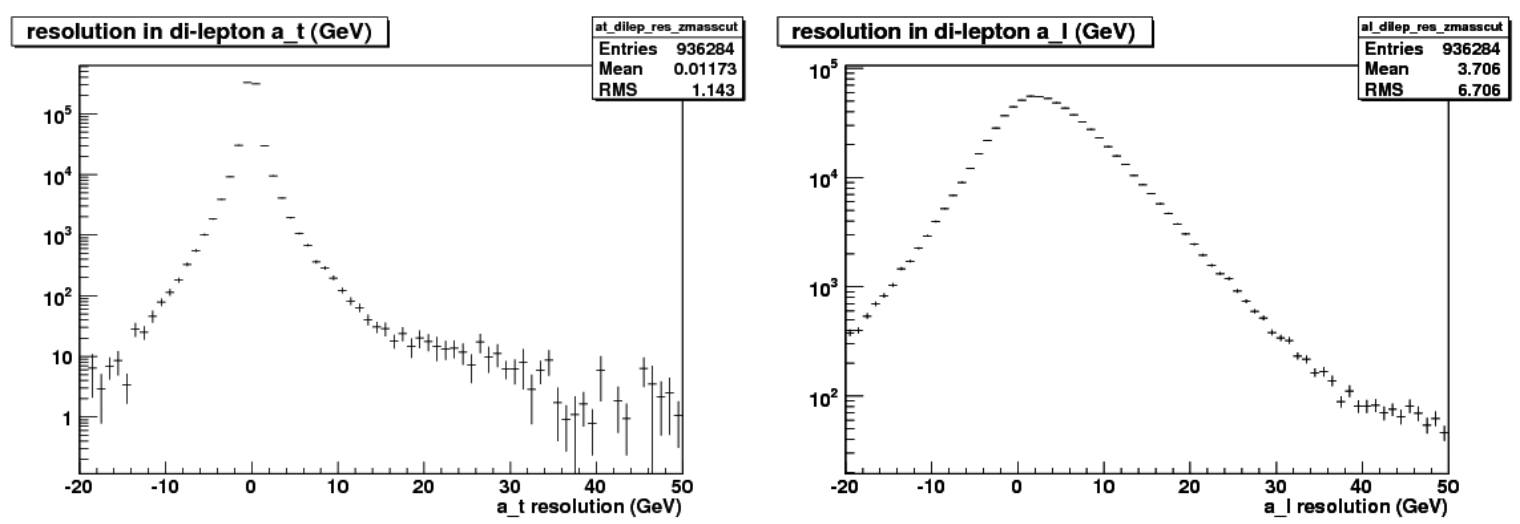

Figure 7.11: Resolution of $a_{t}^{\ell \ell}$ and $a_{l}^{\ell \ell}$ evaluated using MC di-muon events by plotting the difference between the detector level and generator level values of these quantities. Only events with a reconstructed di-muon mass between 70 and $110 \mathrm{GeV}$ are plotted. Keep in mind that the $a_{l}$ component is on average $33 \%$ larger than the $a_{t}$ component. 


\subsubsection{Calorimeter Recoil}

The $\mathbb{E}_{T}$ resolution is intrinsically linked to the energy resolution of reconstructed jets and there are many efforts underway at $\mathrm{D} \varnothing$ aimed at improving the jet energy measurement. Although propagating the improved energy values into the $\mathbb{E}_{T}$ could potentially reduce the mismeasurement tails, we take a different approach. The vector sum of the $p_{T}$ of the jets recoiling against the di-lepton pair is compared to the event $\mathbb{E}_{T}$, and the larger of the two quantities along $a_{l}$ and $a_{t}$ is used to correct the values of $a_{l}^{\ell \ell}$ and $a_{t}^{\ell \ell}$. Because we only consider values which are negative in our coordinate system, and the di-lepton projections are always positive, this correction can only reduce the magnitude of the $a_{l}^{\ell \ell}$ and $a_{t}^{\ell \ell}$ quantities. The calorimeter correction is defined as:

$$
\begin{aligned}
& \delta a_{t}{ }^{c a l}=2 \times \min \left(\Sigma \boldsymbol{p}_{\boldsymbol{T}}{ }^{j e t s} \cdot \hat{a}_{t},-\boldsymbol{H}_{\boldsymbol{T}} \cdot \hat{a}_{t}, 0\right) \\
& \delta a_{l}{ }^{c a l}=2 \times \min \left(\Sigma \boldsymbol{p}_{\boldsymbol{T}}{ }^{j e t s} \cdot \hat{a}_{l},-\boldsymbol{H}_{\boldsymbol{T}} \cdot \hat{a}_{l}, 0\right)
\end{aligned}
$$

in which a jet is used in the $a_{l}\left(a_{t}\right)$ component sum only if it satisfies $\boldsymbol{p}_{T} \cdot \hat{a}_{l}<0\left(\boldsymbol{p}_{T} \cdot \hat{a}_{t}<0\right)$. In so doing we pick the largest possible contribution that the calorimeter may have made to the apparent $\mathbb{E}_{T}$ in the event and the corrections are intrinsically negative. The resolution of these objects is rather broad and the factor of 2 , although not explicitly optimized, accounts for this. The correction term's distribution is shown in Figure 7.12 and Figure 7.13. Of the three corrections, this one has the largest impact on the mismeasured tails. 

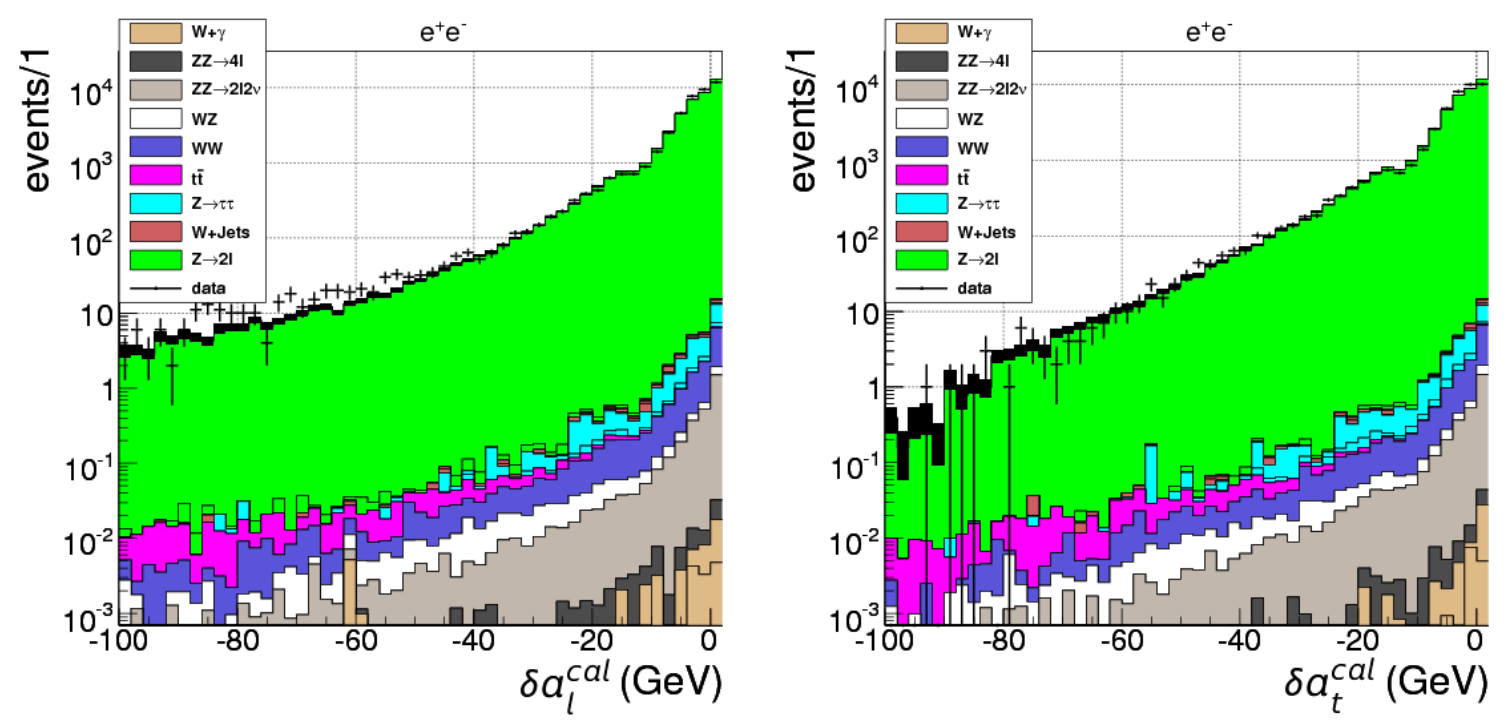

(a) di-electron channel (Run IIa)
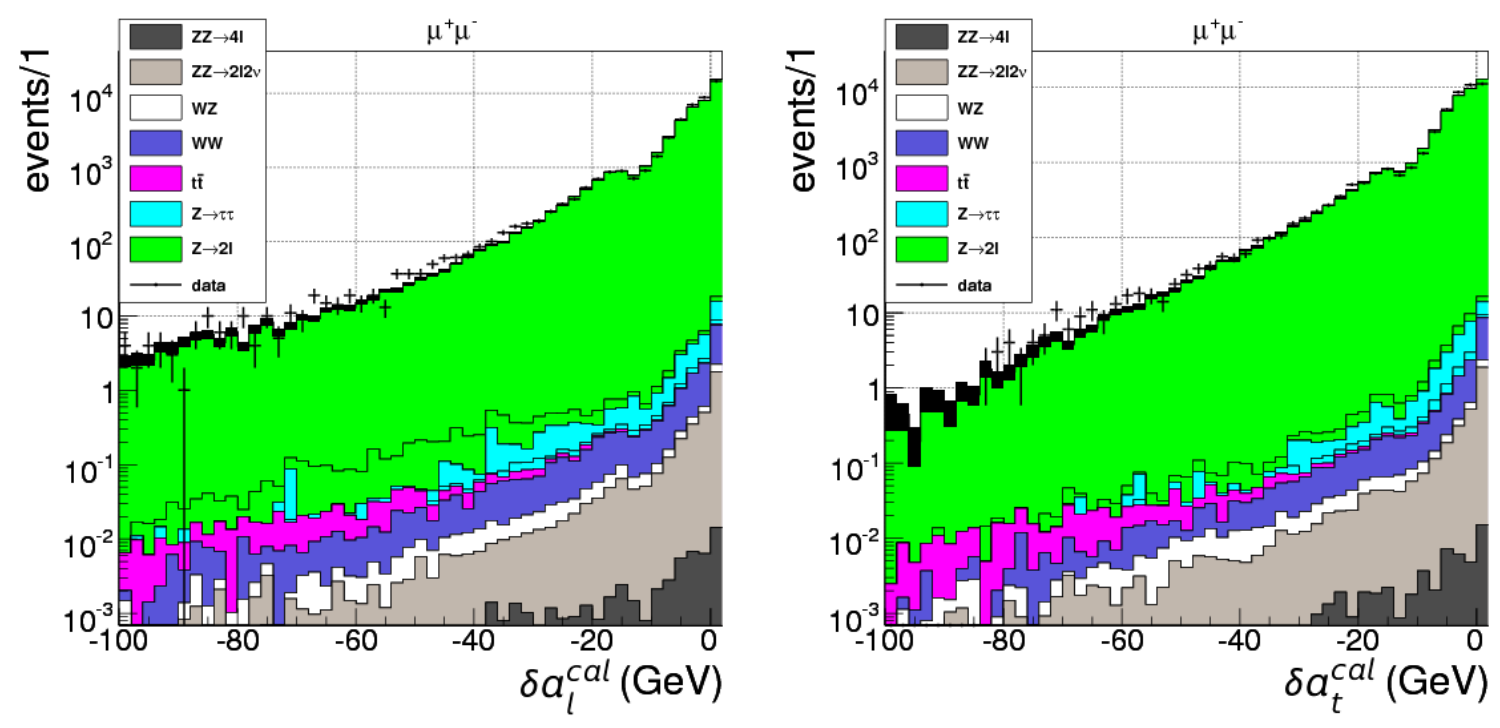

(b) di-muon channel (Run IIa)

Figure 7.12: The calorimeter recoil corrections in Run IIa along the longitudinal direction on the left, and the transverse direction on the right. 

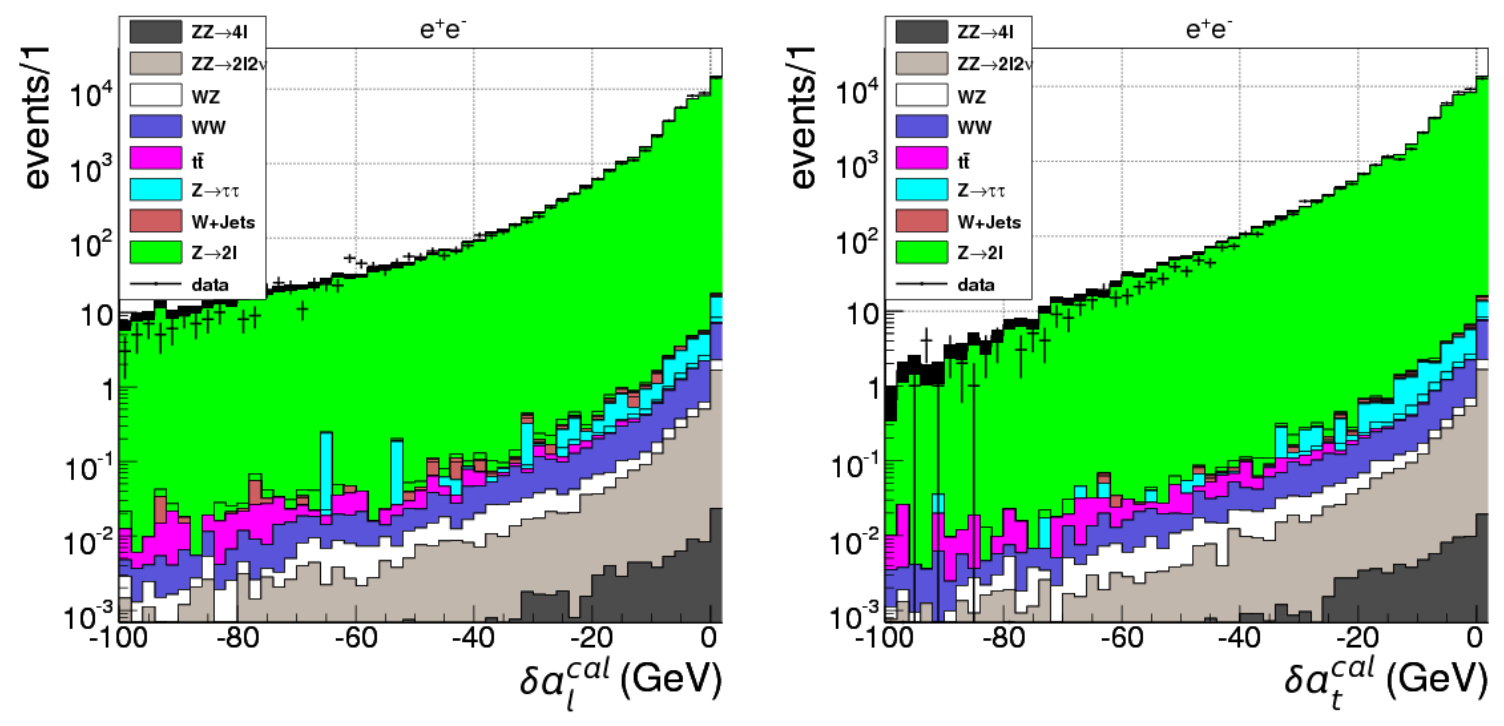

(a) di-electron channel (Run IIa)
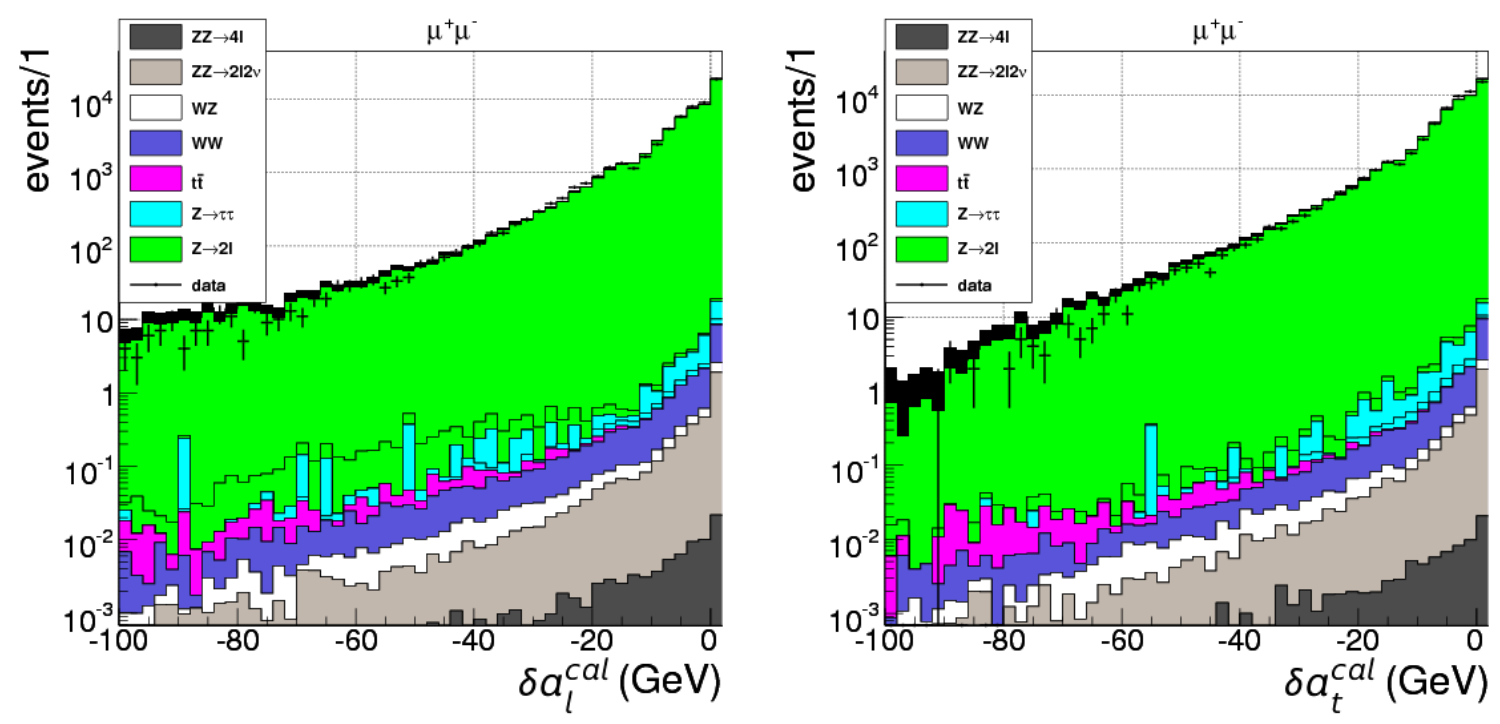

(b) di-muon channel (Run IIa)

Figure 7.13: The calorimeter recoil corrections in Run IIb along the longitudinal direction on the left, and the transverse direction on the right. 


\subsubsection{Recoiling Tracks}

Although rare, lost or unreconstructed jets in the calorimeter are another important source of mismeasured $\mathbb{E}_{T}$. Soft jets which do not penetrate as deeply into the hadronic calorimeter, jets in the inner cryostat region where the energy resolution is degraded, and jets traveling along the fiducial gaps in between the calorimeter cell towers, are all examples of mechanisms through which a jet may fail to satisfy the jet reconstruction requirements, and therefore will not have its full energy recorded or benefit from the jet energy scale corrections. However, the information is not completely lost, and the tracking detector can be used to find such objects, often seen as jets of tracks recoiling against the lepton pair.

It is important not to introduce further problems via our corrections by using poorly measured tracks, or tracks which risk being reconstructed only through algorithmic artifacts rather than the presence of physical particles. The tracks used in this correction must satisfy the following quality cuts:

- Track fit $\chi^{2} / d o f<4.0$

- DCA for tracks with SMT hits $<0.02 \mathrm{~cm}$

- DCA for tracks without SMT hits $<0.2 \mathrm{~cm}$

- $p_{T} \geq 1.0 \mathrm{GeV}$

- $\Delta\left(\operatorname{track}_{z}, P V_{z}\right) \leq 1.0$

- $\Delta R(\operatorname{track}, \ell$ from $Z) \geq 0.5$

- $\Delta R$ (track, calorimeter jet $) \geq 0.5$

Track-jets are built in a manner similar to that of calorimeter jets. First, the track with the largest $p_{T}$ is used as a seed for a new track-jet. All tracks within a $\Delta R \leq 0.5$ cone are added to it and removed from the list of available tracks. This is repeated until every track is associated to one and only one track-jet and every track-jet has at least one track in it.

The $p_{T}$ of these track-jets are projected along $a_{t}$ and $a_{l}$. The contributions of track-jets that are recoiling against the di-lepton pair are then summed, providing us with a measure of the recoiling track activity, $\delta a_{t}{ }^{\text {trk }}$ and $\delta a_{l}{ }^{\text {trk }}$.

$$
\begin{aligned}
\delta a_{t}{ }^{t r k} & =\left(\Sigma \boldsymbol{p}_{T}^{{ }^{t j e t}}\right) \cdot \hat{a}_{t} \\
\delta a_{l}{ }^{t r k} & =\left(\Sigma \boldsymbol{p}_{T}^{t j e t}\right) \cdot \hat{a}_{l} .
\end{aligned}
$$

where a track-jet is included in the correction for the $a_{l}\left(a_{t}\right)$ direction only if it satisfies $\boldsymbol{p}_{T}^{\text {tjet }} \cdot \hat{a}_{l}<$ $0\left(\boldsymbol{p}_{T}^{\text {tjet }} \cdot \hat{a}_{t}<0\right)$. 

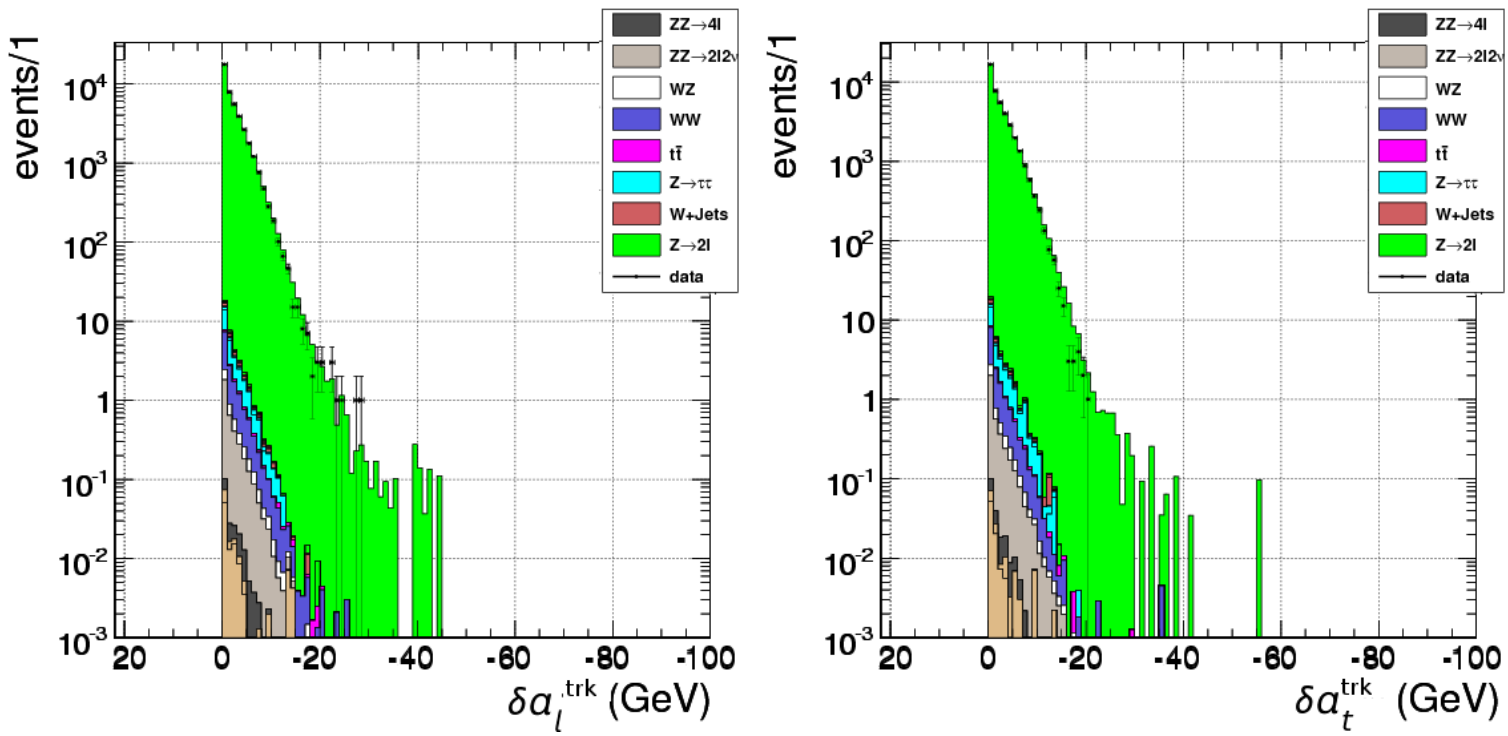

(a) di-electron channel (Run IIa)
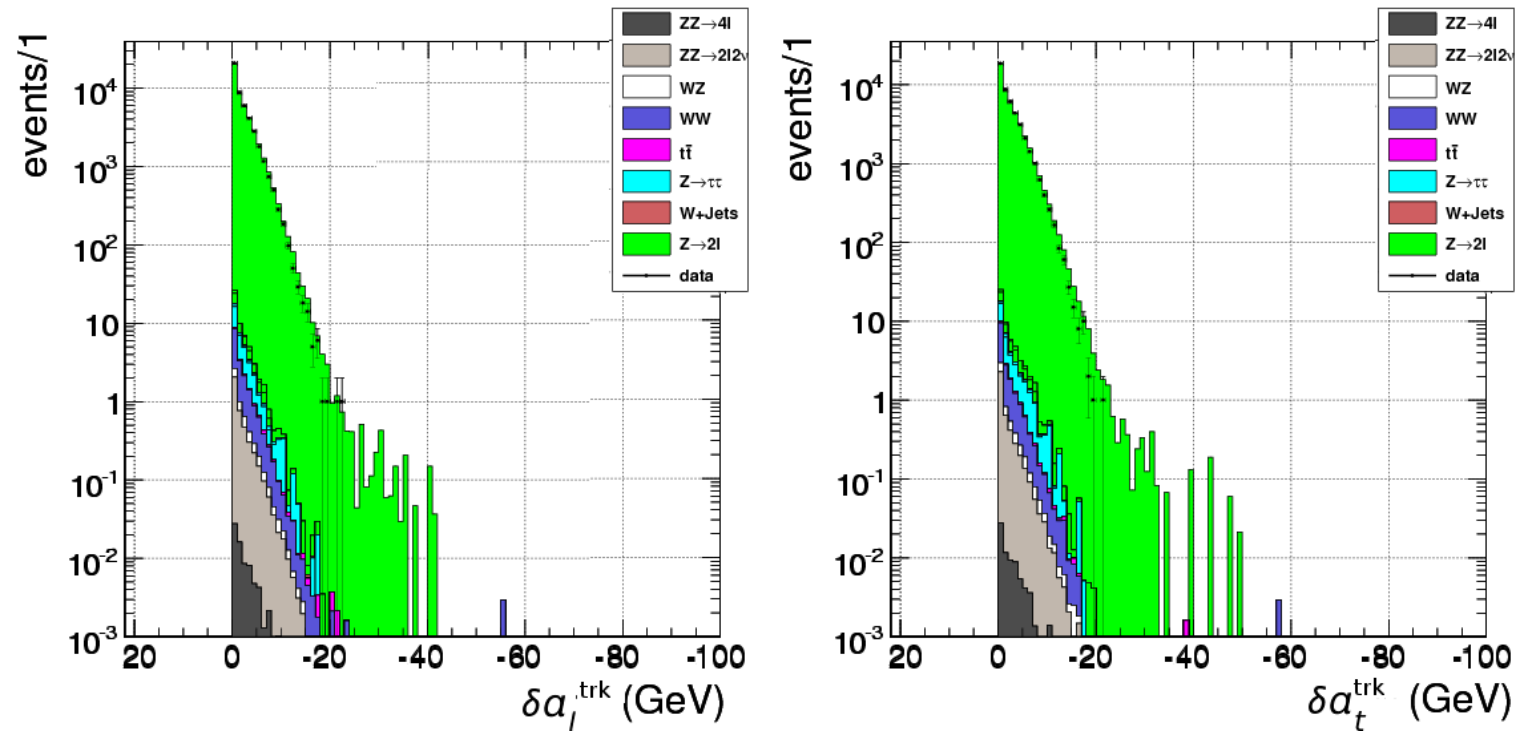

(b) di-muon channel (Run IIa)

Figure 7.14: The track-jet corrections in Run IIa along the longitudinal direction on the left, and the transverse direction on the right. Note that the $\mathrm{x}$-axis runs from zero to negative numbers.

Remember that we have required that the tracks are well separated from the $Z$ candidate leptons and jets in the event. The tracks we have just considered are therefore most likely to be a result of activity in the underlying event or a calorimeter jet which projected along one 

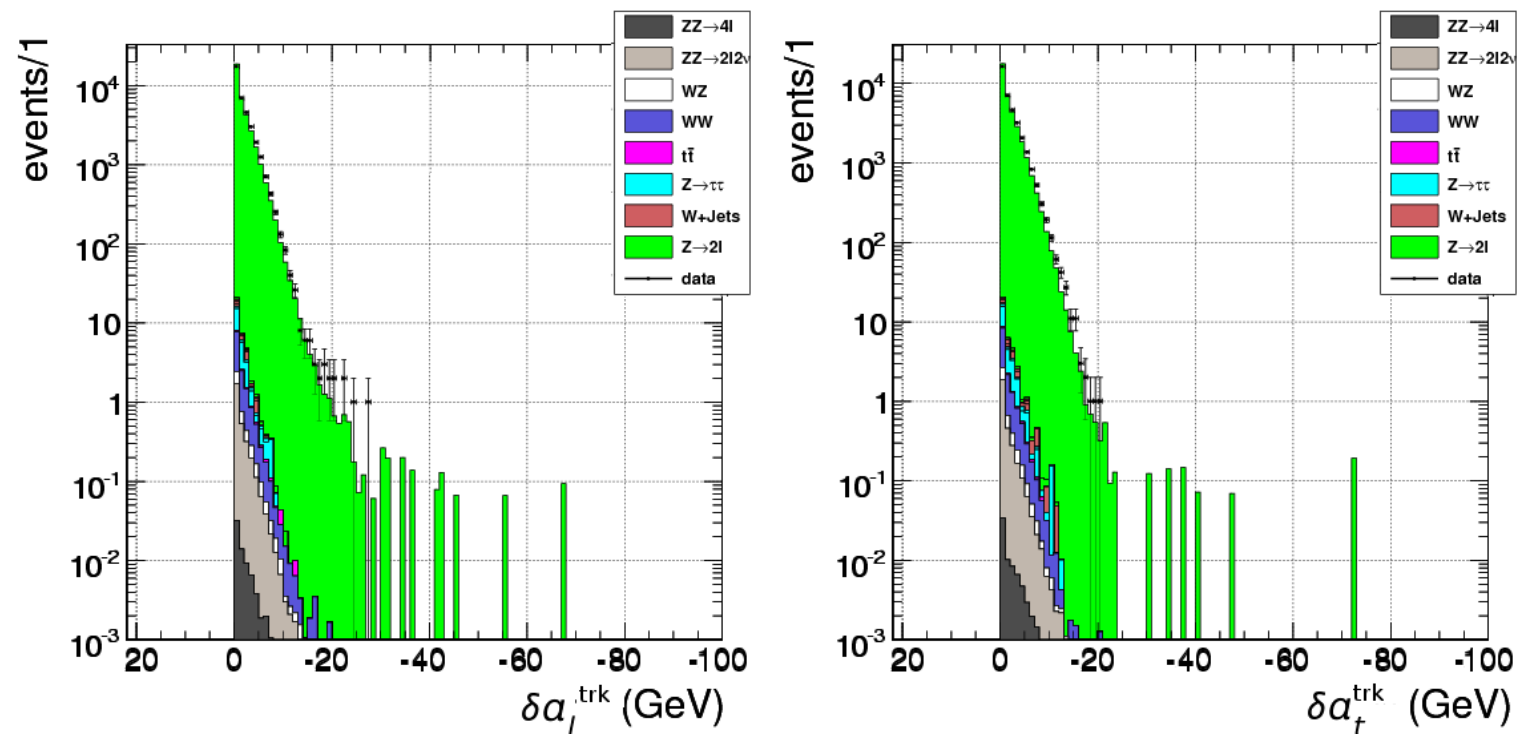

(a) di-electron channel (Run IIb)
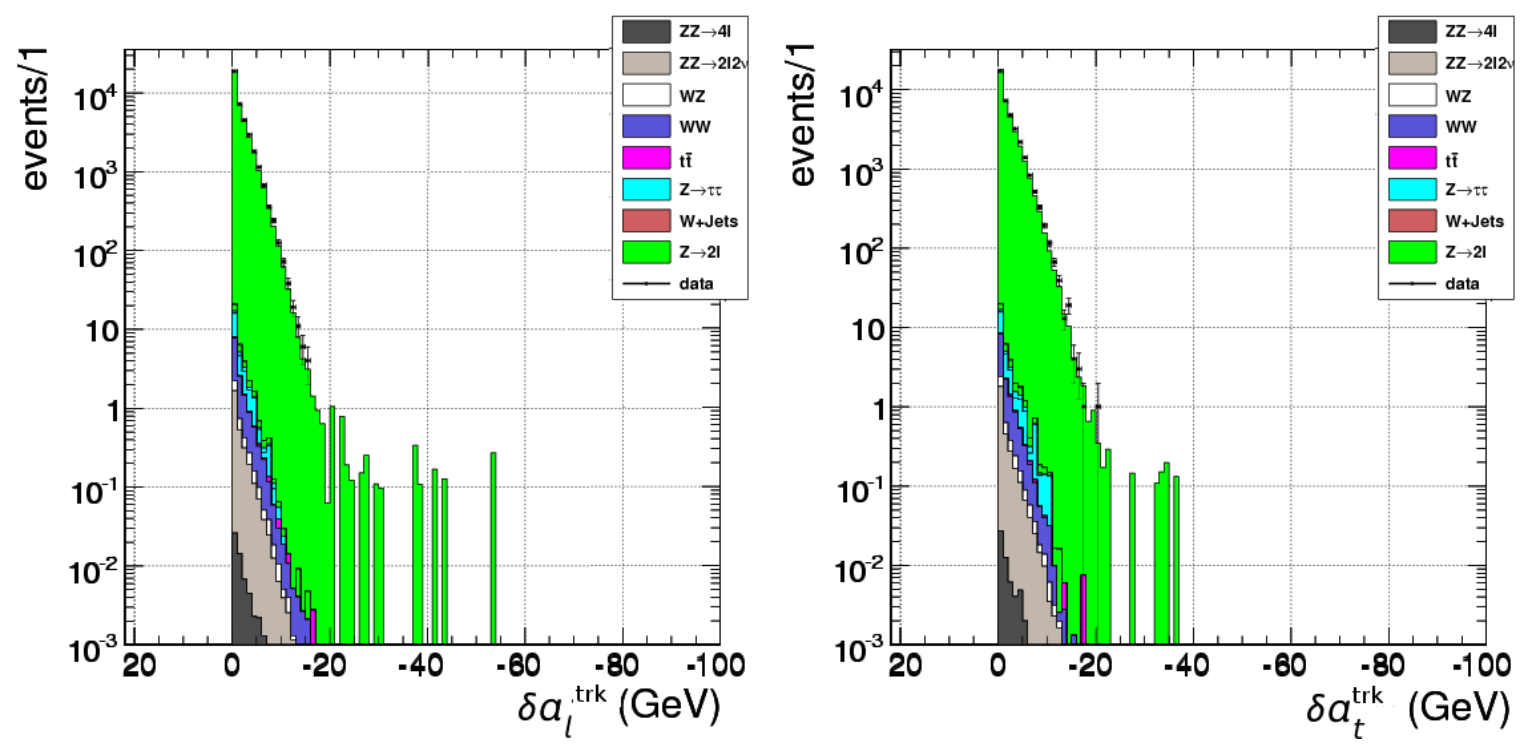

(b) di-muon channel (Run IIb)

Figure 7.15: The track-jet corrections in Run IIb along the longitudinal direction on the left, and the transverse direction on the right. Note that the $\mathrm{x}$-axis runs from zero to negative numbers.

of the poorly instrumented regions of the detector and failed to be reconstructed. In either scenario, this correction has little overlap with the $\delta a_{t}{ }^{c a l}$ and $\delta a_{l}{ }^{c a l}$ from the previous section. 


\subsubsection{Lepton $p_{T}$ uncertainty}

Both of the previous corrections were designed to reduce the sensitivity of $\mathbb{E}_{T}^{\prime}$ to calorimeter effects. Although the lepton $p_{T}$ resolution is, on average, an order of magnitude smaller than that of the jets, there are still cases in which their $p_{T}$ can be vastly mismeasured. For example, the forward regions $(|\eta|>1.6)$ are less well instrumented than the central region, so that the track curvature is at greater risk of being mismeasured. To account for the intrinsic resolution of the lepton $p_{T}$ measurement and further insulate us from these types of effects, the transverse momenta of each of the leptons are individually fluctuated up or down one sigma so as to minimize the di-lepton $p_{T} a_{t}$ and $a_{l}$ components.

The transverse component, $a_{t}^{\ell \ell}$, is minimized by decreasing the transverse momenta of both leptons to give the modified quantity:

$$
a_{t}^{\ell \ell^{\prime}}=\boldsymbol{p}_{T}^{\ell \ell^{\prime}} \cdot \hat{a}_{t}{ }^{\prime}
$$

Here $\boldsymbol{p}_{T}^{\ell \ell^{\prime}}$ and $\hat{a}_{t}{ }^{\prime}$ correspond respectively to $\boldsymbol{p}_{T}^{\ell \ell}$ and $\hat{a}_{t}$, redefined using $\boldsymbol{p}_{T}^{(1)} \times\left(1-\sigma_{1}\right)$ and $\boldsymbol{p}_{T}^{(2)} \times\left(1-\sigma_{2}\right)$ in place of the unscaled quantities and redefining the thrust axis accordingly.

The longitudinal component, $a_{l}{ }^{\ell \ell}$, is minimized by decreasing the value of $\boldsymbol{p}_{T}^{(1)}$ and increasing the value of $\boldsymbol{p}_{T}^{(2)}$ using their fractional uncertainties $\sigma_{1}$ and $\sigma_{2}$ :

$$
\delta a_{t}^{\ell \ell}=a_{t}^{\ell \ell^{\prime}}-a_{t}^{\ell \ell} \quad \delta a_{l}^{\ell \ell}=\left(-\sigma_{1} \boldsymbol{p}_{T}^{(1)}+\sigma_{2} \boldsymbol{p}_{T}^{(2)}\right) \cdot \hat{a}_{l}
$$

If the fractional uncertainty on either of the lepton transverse momenta is larger than unity, then the fractional uncertainties on both $a_{t}^{\ell \ell}$ and $a_{l}^{\ell \ell}$ are set to unity.

Electrons falling at the calorimeter module boundaries ( $\phi$ gap) risk being significantly under-measured. The measurement uncertainties, however, do not reflect this asymmetric bias. If the lower $p_{T}$ electron is within this region, the fractional uncertainty on $a_{l}^{\ell \ell}$ is set to unity. This has the effect of significantly suppressing $a_{l}{ }^{\ell}$ in such cases. 


\subsubsection{Combination of $a_{t}^{\ell \ell}$ and $a_{l}^{\ell \ell}$}

The fully corrected $a_{t}^{\ell \ell}$ and $a_{l}^{\ell \ell}$ components are summed in quadrature, giving more weight to the component less sensitive to the lepton transverse momentum uncertainties. A negative component indicates that there is little to no $\mathbb{E}_{T}$ along that direction and it is dropped from the sum. $\mathbb{E}_{T}^{\prime}$ is a positive definite quantity and we do not want to artificially increase its size when adding negative components in quadrature.

$$
\begin{aligned}
& a_{t}=a_{t}^{\ell \ell}+\delta a_{t}^{c a l}+k^{\prime} \times \delta a_{t}^{\text {trk }}+k \times \delta a_{t}^{\ell \ell} \\
& a_{l}=a_{l}^{\ell \ell}+\delta a_{l}^{c a l}+k^{\prime} \times \delta a_{l}^{t r k}+k \times \delta a_{l}^{\ell \ell}
\end{aligned}
$$

The values of $k$ and $k^{\prime}$ were optimized by maximizing $S / \sqrt{B}$ in a background dominated sample, as described in Appendix C. Recall that by construction the $\delta a_{t}$ and $\delta a_{l}$ terms are always zero or negative while $a_{t}^{\ell \ell}$ and $a_{l}^{\ell \ell}$ are positive. In the case where either $a_{t}$ or $a_{l}$ is negative, its value is zeroed.

The final discriminating variable is then calculated as a weighted quadrature sum of the two components:

$$
\begin{array}{ll}
\text { If } \Delta \phi(\ell, \ell)>\pi / 2 & {E_{T}^{\prime}}_{T}^{\prime}=\sqrt{a_{l}^{2}+\left(1.5 a_{t}\right)^{2}} . \\
\text { If } \Delta \phi(\ell, \ell) \leq \pi / 2 & {E_{T}^{\prime}}^{\prime}=\sqrt{\left(1.5 a_{t}\right)^{2}} .
\end{array}
$$

As previously mentioned, the definition of the variable depends on the size of the opening angle between the two leptons. The decomposition is designed to capitalize on the relative sensitivity of the components to lepton $p_{T}$ mismeasurements. Since $a_{t}$ is largely insensitive to lepton $p_{T}$ mismeasurements, we multiply it by an extra factor of 1.5. As the opening angle decreases, the $a_{l}$ distribution becomes fraught with noise and the component is discarded. The resulting $\not_{T}^{\prime}$ distribution is shown in Figure 7.16.

In this new variable, the Inclusive $Z$ background is confined below $\approx 40 \mathrm{GeV}$, while the samples with genuine $\mathscr{E}_{T}$ are relatively unchanged. This can be seen quite clearly in Figure 7.17, which plots the rejection of samples with artificial $\mathbb{E}_{T}$ versus the acceptance of samples with genuine $\mathbb{E}_{T}$ using the standard $\mathbb{E}_{T}$ definition, as well as $\mathbb{E}_{T}^{\prime}$. For a fixed value of instrumental background rejection, the di-boson selection efficiency can be improved by an order of magnitude.

The cuts on $\mathscr{E}_{T}^{\prime}$ (listed in Table 7.5), were determined by examining the falling edge of the $\mathscr{E}_{T}^{\prime}$ distribution in $\mathrm{MC}$ and selecting a point which removed the bulk of the instrumental backgrounds. Consideration was made to stay as close to the edge as possible while keeping the associated systematics under control, as seen in Figures 7.24, 7.25, 7.26, and 7.27. 

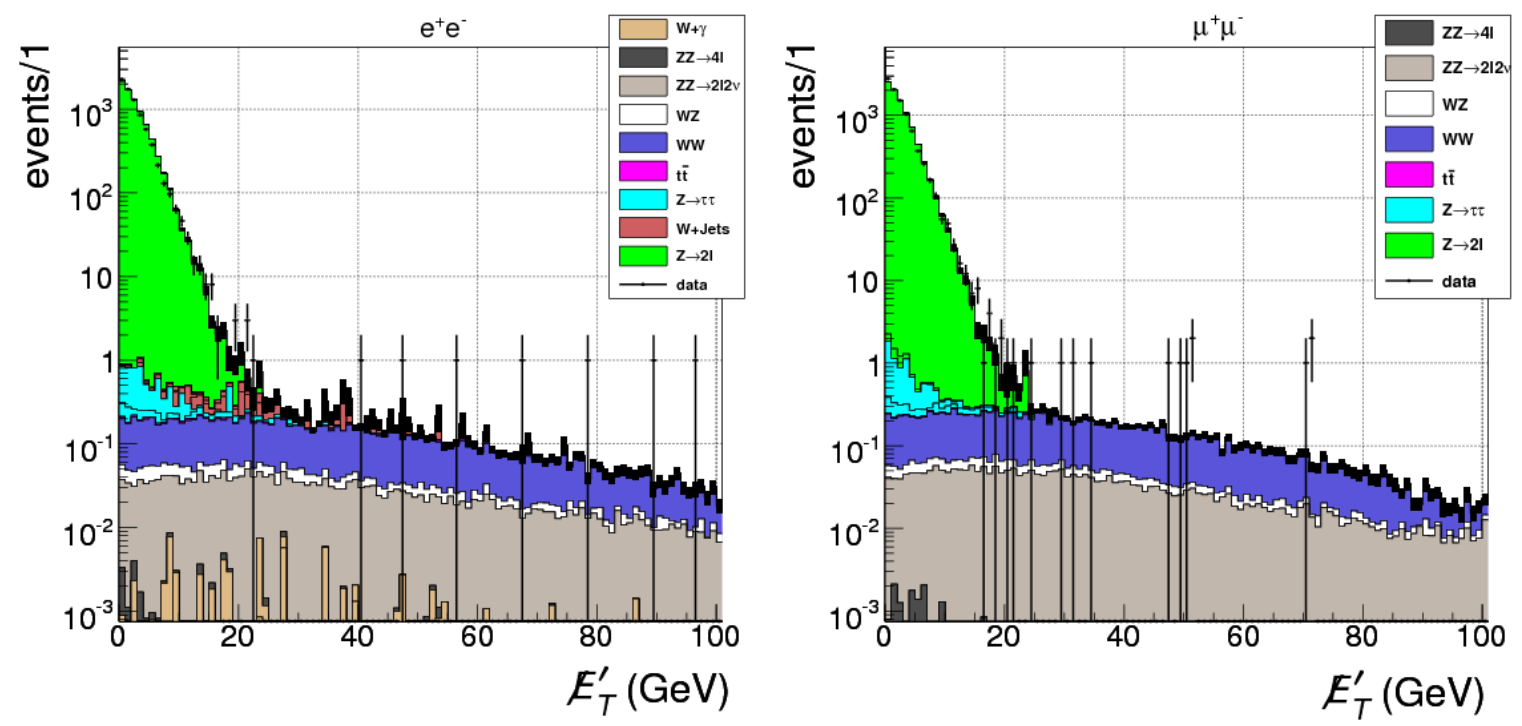

(a) Run IIa
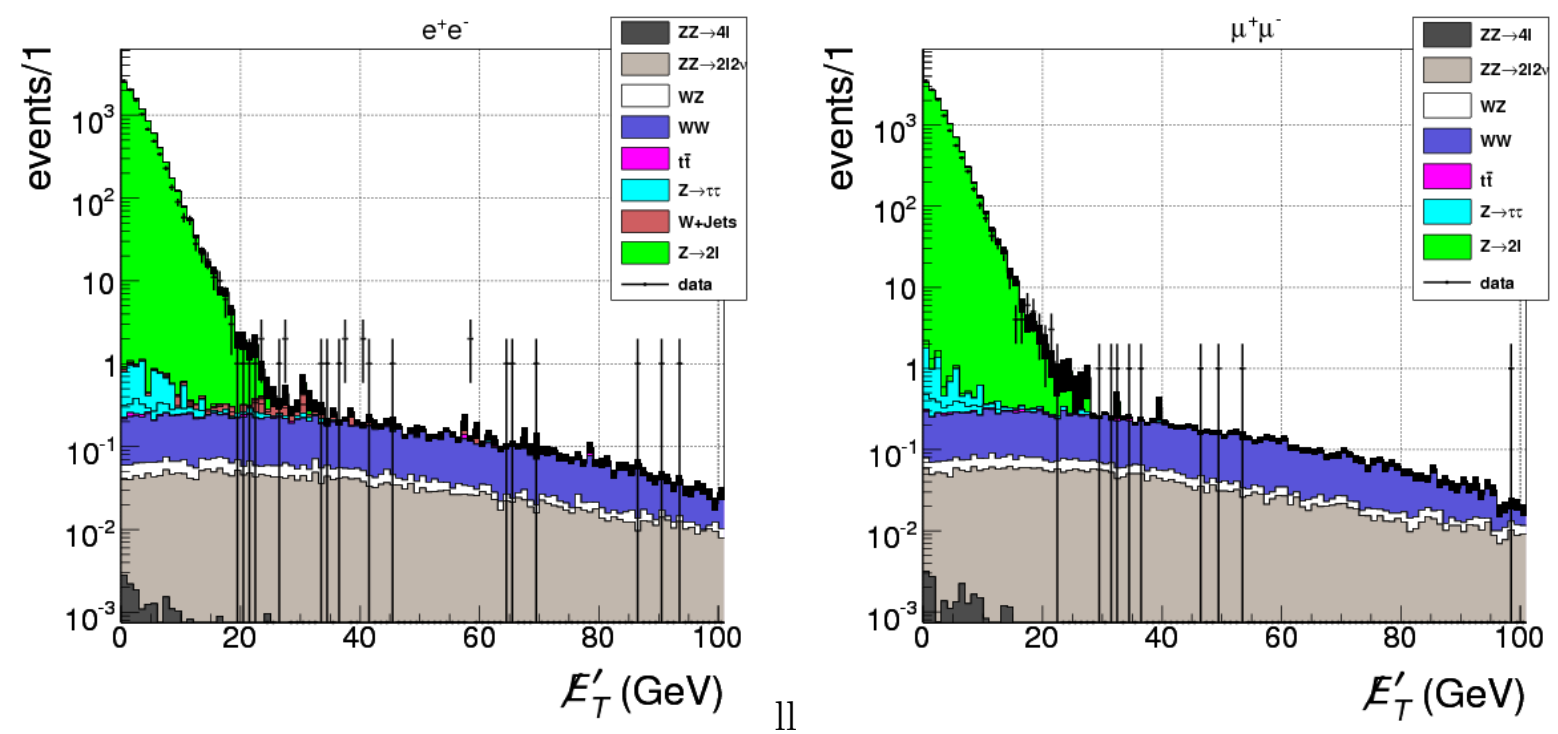

(b) Run IIb

Figure 7.16: The fully corrected instrumental $\mathbb{E}_{T}$ variable $\left(\mathbb{E}_{T}^{\prime}\right)$, of the event evaluated after applying the preselection requirements for the di-electron (left) and di-muon (right) channels. Run IIa is shown in (a) and Run IIb is shown in (b). This distribution can be compared against the $\mathbb{H}_{T}$ distribution in Figure 7.9 . 

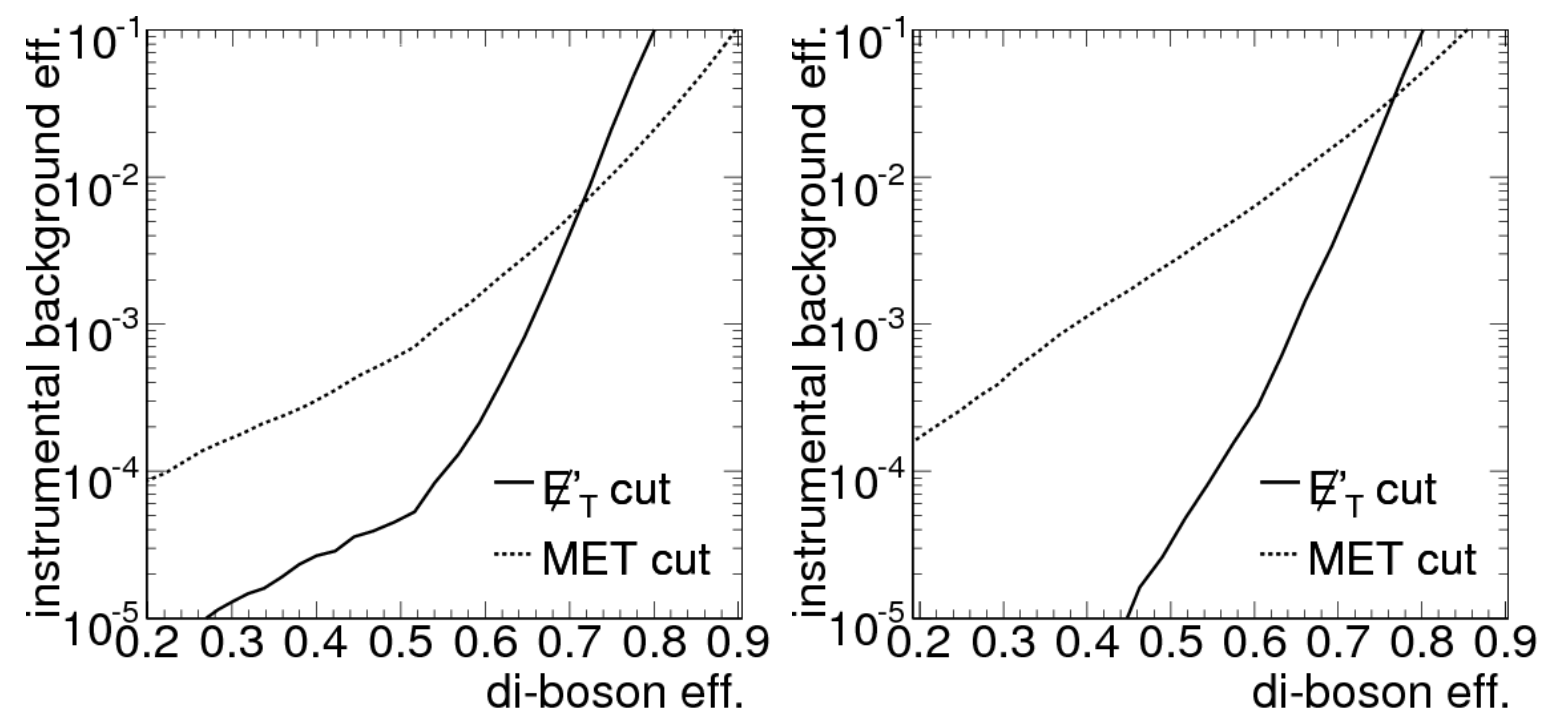

Figure 7.17: The efficiency of $W W, W Z$, and $Z Z$ events vs instrumental background events using the $\mathbb{E}_{T}$ (labeled as MET in the figure) and $\mathbb{E}_{T}^{\prime}$ variables in the di-electron (left) and di-muon (right) channels.

Table 7.5: $\mathbb{E}_{T}^{\prime}$ cut values for the di-electron and di-muon channels in Run IIa and Run IIb

\begin{tabular}{|l|ll|}
\hline \hline & Run IIa & Run IIb \\
\hline di-electron & $27 \mathrm{GeV}$ & $27 \mathrm{GeV}$ \\
di-muon & $30 \mathrm{GeV}$ & $35 \mathrm{GeV}$ \\
\hline
\end{tabular}




\section{$7.5 \quad$ Yields}

We now evaluate the predicted yields from MC and compare them to data. These are shown in Tables 7.6, 7.7, 7.8, and 7.9.

Table 7.6: Number of MC and Data events after the major steps of the selection chain for the di-electron final state (Run IIa). The uncertainties are statistical only.

\begin{tabular}{|c|c|c|c|c|c|c|}
\hline Samples & Pre-cuts & di-lepton sel & Jet Veto & Extra Activity & Charge & $\mathbb{E}_{T}^{\prime}$ \\
\hline$Z \rightarrow \ell \ell$ & $58702.45 \pm 76.4$ & $53774.14 \pm 71.5$ & $53111.18 \pm 70.9$ & $43459.87 \pm 63.2$ & $42940.04 \pm 62.8$ & $0.04 \pm 0.0$ \\
\hline$Z \rightarrow \tau \tau$ & $233.80 \pm 3.4$ & $21.21 \pm 1.0$ & $21.06 \pm 1.0$ & $17.52 \pm 0.9$ & $17.24 \pm 0.9$ & $0.13 \pm 0.0$ \\
\hline $\mathrm{W}+$ Jets & $33.97 \pm 1.4$ & $11.39 \pm 0.8$ & $11.18 \pm 0.8$ & $8.50 \pm 0.7$ & $5.23 \pm 0.6$ & $1.64 \pm 0.3$ \\
\hline$W+\gamma$ & $5.94 \pm 0.1$ & $2.00 \pm 0.1$ & $1.98 \pm 0.1$ & $1.66 \pm 0.1$ & $0.05 \pm 0.0$ & $0.01 \pm 0.0$ \\
\hline$t \bar{t}$ & $17.04 \pm 0.2$ & $6.83 \pm 0.1$ & $4.98 \pm 0.1$ & $0.96 \pm 0.0$ & $0.95 \pm 0.0$ & $0.15 \pm 0.0$ \\
\hline WW & $32.82 \pm 0.2$ & $12.57 \pm 0.1$ & $12.40 \pm 0.1$ & $10.42 \pm 0.1$ & $10.35 \pm 0.1$ & $4.76 \pm 0.1$ \\
\hline WZ & $10.13 \pm 0.1$ & $8.24 \pm 0.1$ & $7.97 \pm 0.1$ & $1.30 \pm 0.0$ & $1.19 \pm 0.0$ & $0.45 \pm 0.0$ \\
\hline$Z Z \rightarrow \ell \ell \ell \ell$ & $1.44 \pm 0.0$ & $1.22 \pm 0.0$ & $1.13 \pm 0.0$ & $0.07 \pm 0.0$ & $0.06 \pm 0.0$ & $0.01 \pm 0.0$ \\
\hline$Z Z \rightarrow \ell \ell \nu \nu$ & $4.07 \pm 0.0$ & $3.85 \pm 0.0$ & $3.80 \pm 0.0$ & $3.20 \pm 0.0$ & $3.17 \pm 0.0$ & $1.75 \pm 0.0$ \\
\hline Tot bckg & $59031.65 \pm 76.5$ & $53835.59 \pm 71.5$ & $53169.90 \pm 70.9$ & $43498.64 \pm 63.2$ & $42975.07 \pm 62.9$ & $7.17 \pm 0.3$ \\
\hline Tot MC & $59035.72 \pm 76.5$ & $53839.43 \pm 71.5$ & $53173.70 \pm 70.9$ & $43501.84 \pm 63.2$ & $42978.24 \pm 62.9$ & $8.92 \pm 0.3$ \\
\hline Data & $59099.00 \pm 243.1$ & $53255.00 \pm 230.8$ & $52562.00 \pm 229.3$ & $44163.00 \pm 210.1$ & $42973.00 \pm 207.3$ & $8.00 \pm 2.8$ \\
\hline
\end{tabular}

Table 7.7: Number of MC and Data events after the major steps of the selection chain for the di-muon final state (Run IIa). The uncertainties are statistical only.

\begin{tabular}{|lcccccc|}
\hline \hline Samples & Pre-cuts & di-lepton sel & Jet Veto & Extra Activity & Charge \\
\hline$Z \rightarrow \ell \ell$ & $64562.41 \pm 84.8$ & $56675.57 \pm 76.6$ & $55762.64 \pm 75.7$ & $45368.61 \pm 67.2$ & $45368.61 \pm 67.2$ & $0.00 \pm 0.0$ \\
$Z \rightarrow \tau \tau$ & $271.66 \pm 3.9$ & $25.59 \pm 1.0$ & $24.97 \pm 1.0$ & $20.67 \pm 0.9$ & $20.67 \pm 0.9$ & $0.07 \pm 0.0$ \\
$t \bar{t}$ & $20.62 \pm 0.2$ & $7.12 \pm 0.1$ & $4.78 \pm 0.1$ & $0.90 \pm 0.0$ & $0.90 \pm 0.0$ & $0.08 \pm 0.0$ \\
$\mathrm{WW}$ & $41.96 \pm 0.2$ & $14.60 \pm 0.1$ & $14.31 \pm 0.1$ & $12.03 \pm 0.1$ & $12.03 \pm 0.1$ & $4.82 \pm 0.1$ \\
$\mathrm{WZ}$ & $10.37 \pm 0.1$ & $8.40 \pm 0.1$ & $7.91 \pm 0.1$ & $1.13 \pm 0.0$ & $1.13 \pm 0.0$ & $0.38 \pm 0.0$ \\
$Z Z \rightarrow \ell \ell \ell \ell$ & $1.59 \pm 0.0$ & $1.31 \pm 0.0$ & $1.06 \pm 0.0$ & $0.06 \pm 0.0$ & $0.06 \pm 0.0$ & $0.00 \pm 0.0$ \\
$Z Z \rightarrow \ell \ell \nu \nu$ & $5.02 \pm 0.0$ & $4.34 \pm 0.0$ & $4.26 \pm 0.0$ & $3.60 \pm 0.0$ & $3.60 \pm 0.0$ & $1.66 \pm 0.0$ \\
Tot bckg & $64908.62 \pm 84.8$ & $56732.59 \pm 76.6$ & $55815.68 \pm 75.7$ & $45403.40 \pm 67.2$ & $45403.40 \pm 67.2$ & $5.35 \pm 0.1$ \\
Tot MC & $64913.64 \pm 84.8$ & $56736.93 \pm 76.6$ & $55819.95 \pm 75.7$ & $45407.00 \pm 67.2$ & $45407.00 \pm 67.2$ & $7.01 \pm 0.1$ \\
\hline Data & $63631.00 \pm 252.3$ & $55049.00 \pm 234.6$ & $54081.00 \pm 232.6$ & $45407.00 \pm 213.1$ & $45407.00 \pm 213.1$ & $10.00 \pm 3.2$ \\
\hline \hline
\end{tabular}


Table 7.8: Number of MC and Data events after the major steps of the selection chain for the di-electron final state (Run IIb). The uncertainties are statistical only.

\begin{tabular}{|lcccccc|}
\hline \hline Samples & Pre-cuts & di-lepton sel & Jet Veto & Extra Activity & Charge \\
\hline$Z \rightarrow \ell \ell$ & $70663.84 \pm 83.3$ & $64693.54 \pm 78.2$ & $64135.85 \pm 77.8$ & $51010.41 \pm 68.2$ & $50518.75 \pm 67.9$ & $0.43 \pm 0.2$ \\
W Jets & $20.37 \pm 0.7$ & $6.85 \pm 0.4$ & $6.75 \pm 0.4$ & $4.97 \pm 0.3$ & $2.93 \pm 0.3$ & $1.08 \pm 0.2$ \\
$Z \rightarrow \tau \tau$ & $273.98 \pm 6.6$ & $27.04 \pm 2.0$ & $26.28 \pm 1.9$ & $21.16 \pm 1.7$ & $20.99 \pm 1.7$ & $0.22 \pm 0.0$ \\
$t \bar{t}$ & $25.90 \pm 0.4$ & $9.53 \pm 0.2$ & $7.36 \pm 0.2$ & $1.12 \pm 0.1$ & $1.11 \pm 0.1$ & $0.19 \pm 0.0$ \\
$\mathrm{WW}$ & $43.76 \pm 0.2$ & $15.47 \pm 0.1$ & $15.31 \pm 0.1$ & $12.58 \pm 0.1$ & $12.52 \pm 0.1$ & $5.84 \pm 0.1$ \\
$\mathrm{WZ}$ & $13.85 \pm 0.1$ & $11.00 \pm 0.1$ & $10.72 \pm 0.1$ & $1.64 \pm 0.0$ & $1.52 \pm 0.0$ & $0.63 \pm 0.0$ \\
$Z Z \rightarrow \ell \ell \ell \ell$ & $2.09 \pm 0.0$ & $1.72 \pm 0.0$ & $1.60 \pm 0.0$ & $0.10 \pm 0.0$ & $0.09 \pm 0.0$ & $0.01 \pm 0.0$ \\
$Z Z \rightarrow \ell \ell \nu \nu$ & $5.37 \pm 0.0$ & $5.00 \pm 0.0$ & $4.95 \pm 0.0$ & $4.03 \pm 0.0$ & $4.01 \pm 0.0$ & $2.28 \pm 0.0$ \\
Tot bckg & $71043.79 \pm 83.6$ & $64765.15 \pm 78.3$ & $64203.86 \pm 77.8$ & $51051.98 \pm 68.2$ & $50557.92 \pm 67.9$ & $8.40 \pm 0.3$ \\
Tot MC & $71049.16 \pm 83.6$ & $64770.14 \pm 78.3$ & $64208.80 \pm 77.8$ & $51056.02 \pm 68.2$ & $50561.93 \pm 67.9$ & $10.68 \pm 0.3$ \\
\hline Data & $72435.00 \pm 269.1$ & $65595.00 \pm 256.1$ & $64668.00 \pm 254.3$ & $51844.00 \pm 227.7$ & $50559.00 \pm 224.9$ & $20.00 \pm 4.5$ \\
\hline \hline
\end{tabular}

Table 7.9: Number of MC and Data events after the major steps of the selection chain for the di-muon final state (Run IIb). The uncertainties are statistical only.

\begin{tabular}{|lcccccc}
\hline \hline Samples & Pre-cuts & di-lepton sel & Jet Veto & Extra Activity & Charge \\
\hline$Z \rightarrow \ell \ell$ & $82138.36 \pm 127.6$ & $73615.24 \pm 120.2$ & $72855.28 \pm 119.3$ & $57669.79 \pm 104.4$ & $57669.79 \pm 104.4$ & $0.11 \pm 0.1$ \\
$Z \rightarrow \tau \tau$ & $274.56 \pm 6.3$ & $27.72 \pm 1.9$ & $27.11 \pm 1.8$ & $22.21 \pm 1.7$ & $22.21 \pm 1.7$ & $0.02 \pm 0.0$ \\
$t \bar{t}$ & $24.12 \pm 0.4$ & $8.84 \pm 0.2$ & $6.53 \pm 0.2$ & $1.14 \pm 0.1$ & $1.14 \pm 0.1$ & $0.13 \pm 0.0$ \\
$\mathrm{WW}$ & $49.19 \pm 0.2$ & $17.43 \pm 0.1$ & $17.22 \pm 0.1$ & $14.08 \pm 0.1$ & $14.08 \pm 0.1$ & $4.83 \pm 0.1$ \\
$\mathrm{WZ}$ & $11.93 \pm 0.1$ & $9.94 \pm 0.1$ & $9.52 \pm 0.1$ & $1.55 \pm 0.0$ & $1.55 \pm 0.0$ & $0.44 \pm 0.0$ \\
$Z Z \rightarrow \ell \ell \ell \ell$ & $1.87 \pm 0.0$ & $1.58 \pm 0.0$ & $1.34 \pm 0.0$ & $0.09 \pm 0.0$ & $0.09 \pm 0.0$ & $0.00 \pm 0.0$ \\
$Z Z \rightarrow \ell \ell \nu \nu$ & $5.76 \pm 0.0$ & $5.14 \pm 0.0$ & $5.08 \pm 0.0$ & $4.16 \pm 0.0$ & $4.16 \pm 0.0$ & $1.73 \pm 0.0$ \\
Tot bckg & $82500.05 \pm 127.7$ & $73680.76 \pm 120.2$ & $72917.01 \pm 119.4$ & $57708.84 \pm 104.4$ & $57708.84 \pm 104.4$ & $5.54 \pm 0.1$ \\
Tot MC & $82505.81 \pm 127.7$ & $73685.90 \pm 120.2$ & $72922.09 \pm 119.4$ & $57713.00 \pm 104.4$ & $57713.00 \pm 104.4$ & $7.27 \pm 0.1$ \\
\hline Data & $82989.00 \pm 288.1$ & $72911.00 \pm 270.0$ & $71707.00 \pm 267.8$ & $57713.00 \pm 240.2$ & $57713.00 \pm 240.2$ & $5.00 \pm 2.2$ \\
\hline \hline
\end{tabular}




\subsection{Likelihood Background Discriminant ${ }^{3}$}

The main analysis concern so far has been the elimination of events in our preselection sample, primarily from inclusive $Z$ production, with artificially high $\mathbb{E}_{T}$. There are however a number of backgrounds which have genuine $\mathbb{E}_{T}$, such as the $W W$ and $W+$ jets. As shown in Tables 7.6, 7.7, 7.8, and 7.9, these backgrounds represent an important contribution to the final sample after the cut on $\mathbb{E}_{T}^{\prime}$. To further distinguish between these samples and the signal, we use a likelihood discriminant. It is identical in construction to the one used for the selection of electrons in Equation 4.10 with the following variables considered as inputs:

- Invariant mass of the di-lepton system $M_{\ell \ell}$ (for the di-electron channel)

- $\chi^{2}$ probability from a re-fit of the momenta of the muons from the $Z$ decay, subject to the constraints of the initial momentum values and that the di-muon invariant mass is consistent with the $Z$ mass (for the di-muon channel)

- Transverse momentum of the leading lepton $p_{T}^{\text {lead }-\ell}$

- Cosine of the scattering angle of the negative lepton in the di-lepton rest frame $\cos \left(\theta^{*}\right)$. The Collins-Soper frame is used: it is defined as the frame where the z-axis bisects the angle formed by the momentum of the proton and the negative-momentum of the anti-proton

- Opening angle between the di-lepton system and the leading lepton $\Delta \phi\left(\ell_{\text {lead }}, Z\right)$

The di-lepton mass is replaced with a $\chi^{2}$ probability in the muon channel. It is obtained by minimizing the following $\chi^{2}$ function with respect to $\frac{1}{p_{T^{f i t}}}$ :

$$
\chi^{2}\left(1 / p_{T 1}\right)=\left(\frac{1 / p_{T_{1}}^{o b s}-1 / p_{T 1}^{f i t}}{\sigma\left(1 / p_{T 1}\right)}\right)^{2}+\left(\frac{1 / p_{T_{2}}^{o b s}-1 / p_{T 2}^{f i t}}{\sigma\left(1 / p_{T 2}\right)}\right)^{2},
$$

The function is minimized using MINUIT imposing the mass constraint:

$$
p_{2}^{f i t}=\frac{M_{Z}^{2}}{2[1-\cos \alpha] p_{1}^{f i t}},
$$

where $\alpha$ is the opening angle between the two muons. The uncertainties on the measured transverse momenta are obtained from the transfer functions described in Section B.1. The distribution of the $\chi^{2}$ function for signal and background is shown in Figure 7.18. As expected, the signal distribution behaves like a $\chi^{2}$ for 1 dof. The corresponding probability is therefore used as input to the likelihood discriminant.

\footnotetext{
${ }^{3}$ The implementation of the likelihood method was performed by Gianluca Cerminara
} 

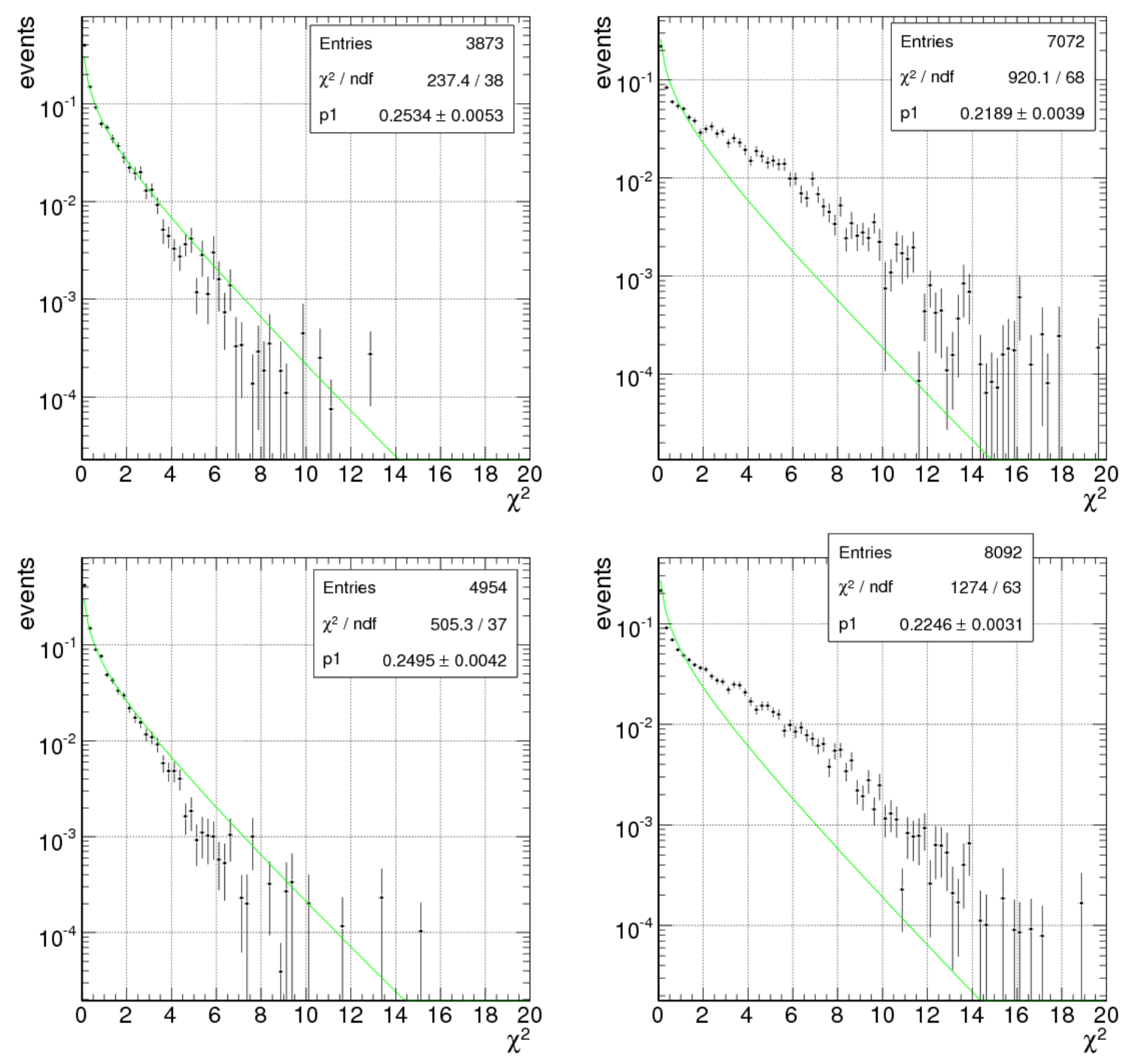

Figure 7.18: Distribution of the $\chi^{2}$ described by Equation 7.7 for signal (left) and background (right) MC events. The plots at the top refer to the Run IIa dataset while the plots at the bottom refer to Run IIb. The green line represent the fit with a $\chi^{2}$ function for 1 d.o.f.

The likelihood is built by splitting the MC into two individual sub-samples. The first is used to fit the $P(x)$ functions, the second for the evaluation of the likelihood, and all the rest of the analysis steps. For some of the samples, namely the $W+j e t s$, the MC sample was too small to split and maintain a reasonable control on the statistical uncertainty. This sample was ignored in the training of the likelihood. The input variables are shown in Figures 7.19, 7.20, 7.21, and 7.22. The binned output of this likelihood variable, shown in Figure 7.23, is used 
to estimate the sensitivity of the search and the $Z Z$ production cross section according to the prescription described in Chapter 6.
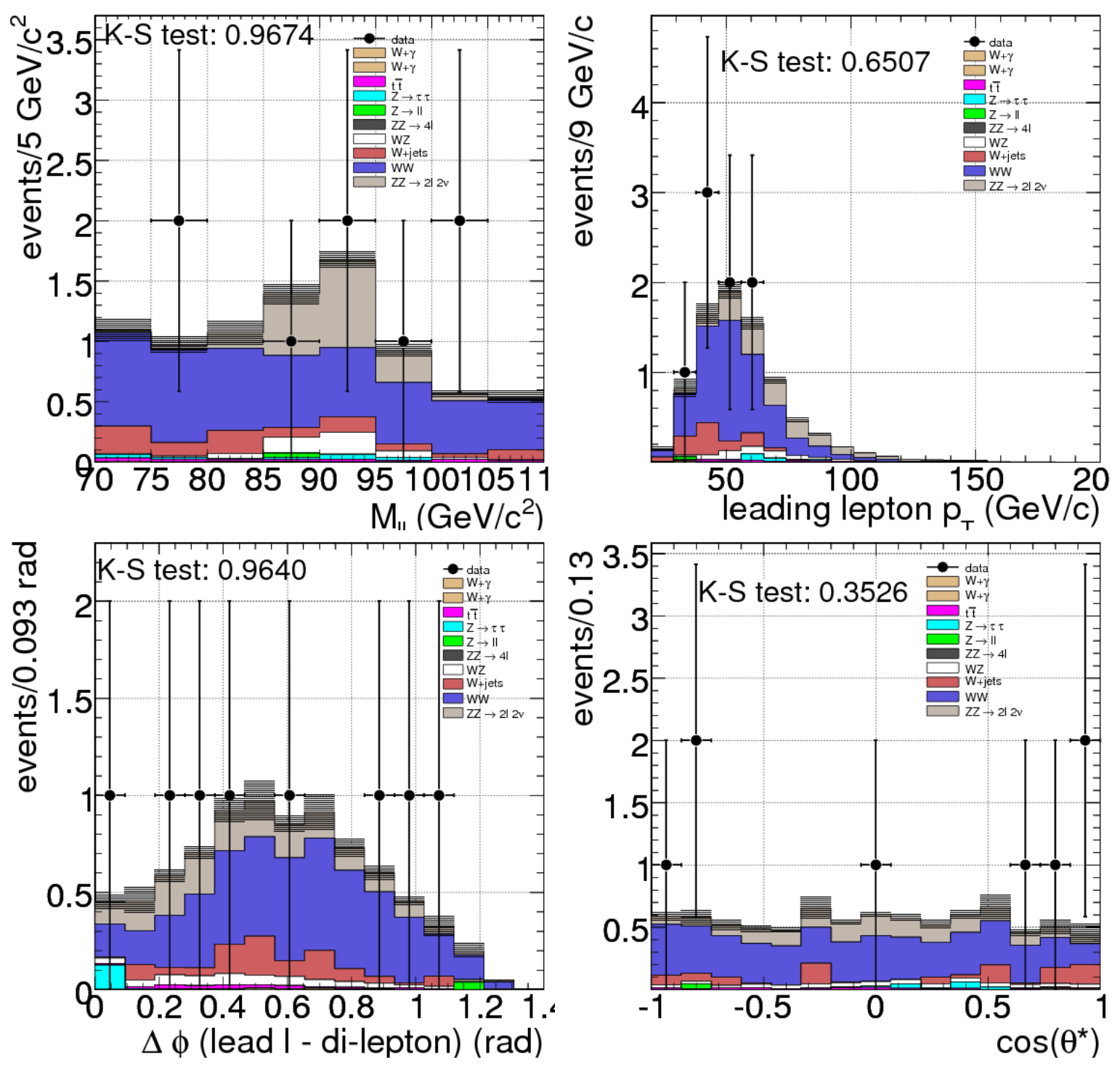

Figure 7.19: Distribution of the input variables of the likelihood discriminant for data and MC in the di-electron channel (Run IIa). 

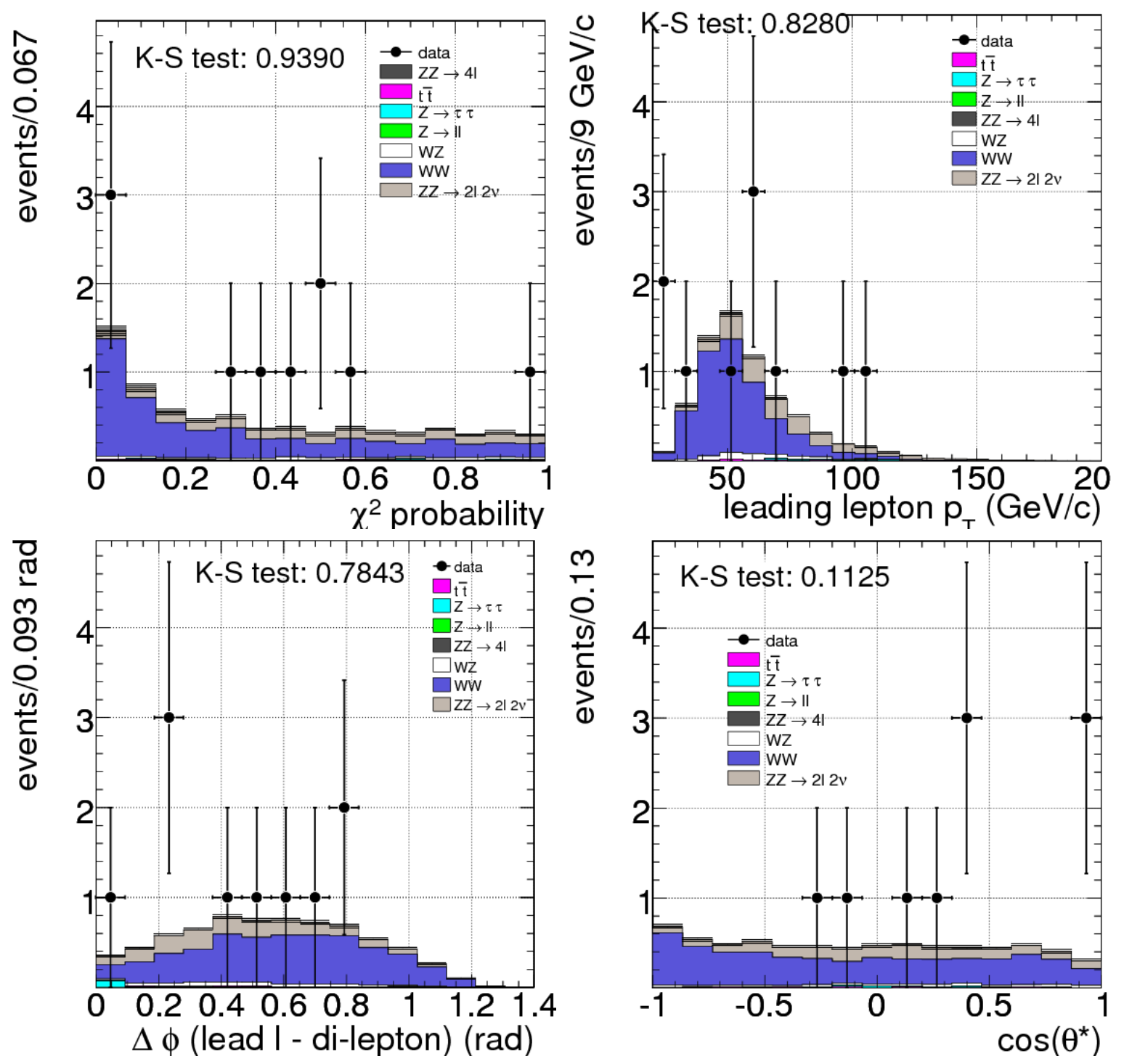

Figure 7.20: Distribution of the input variables of the likelihood discriminant for data and MC in the di-muon channel (Run IIa). 

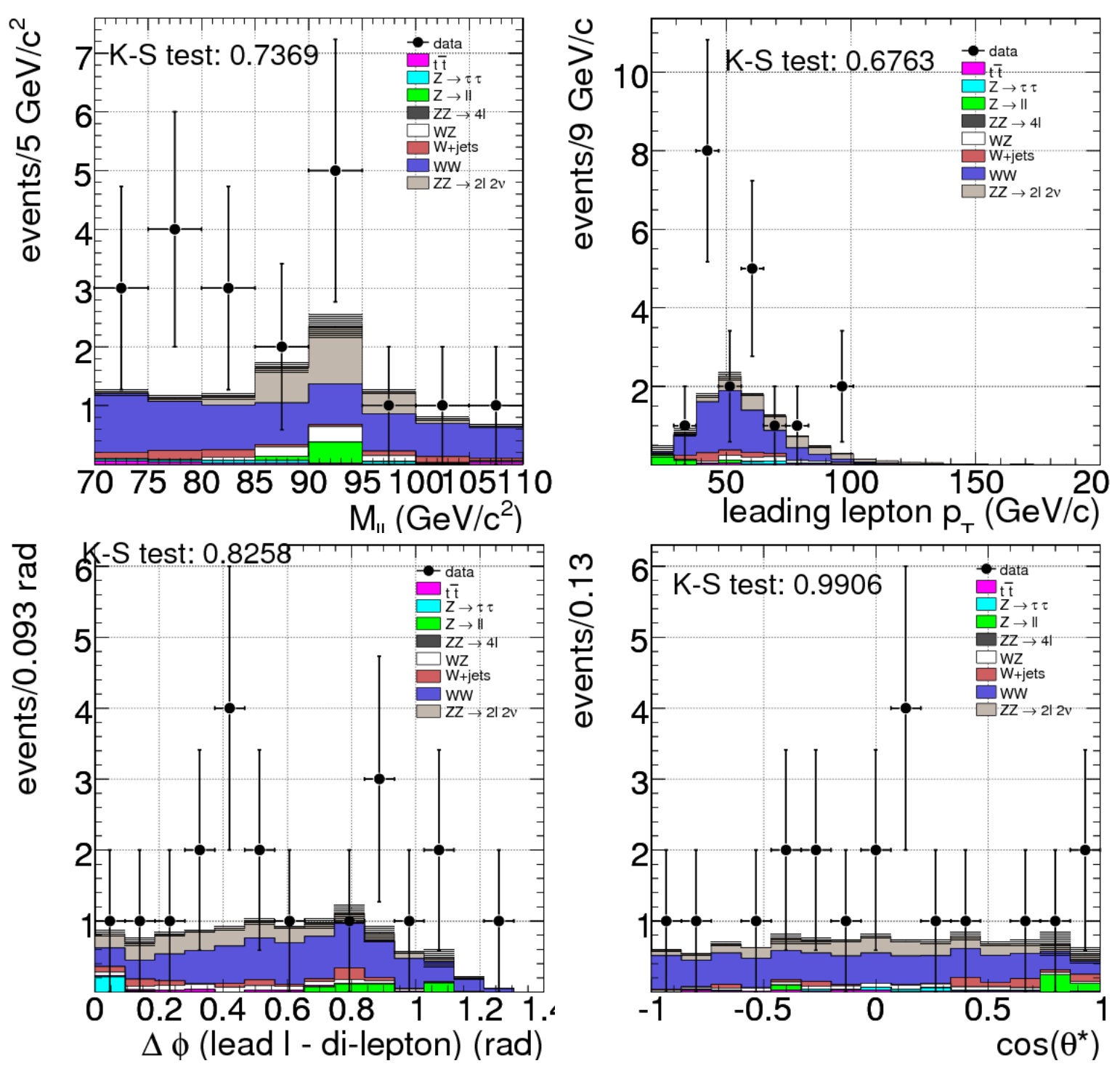

Figure 7.21: Distribution of the input variables of the likelihood discriminant for data and MC in the di-electron channel (Run IIb). 

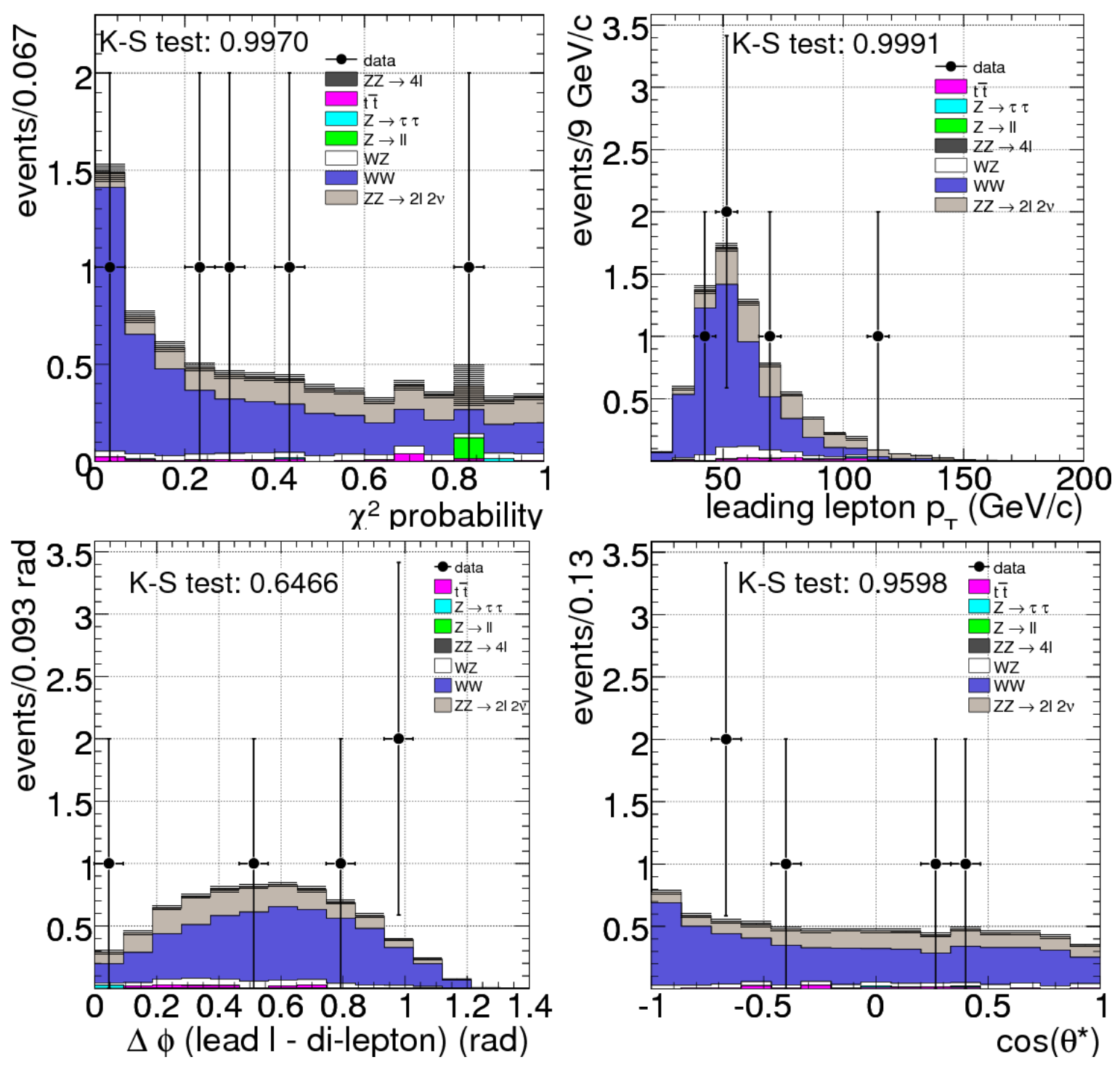

Figure 7.22: Distribution of the input variables of the likelihood discriminant for data and MC in the di-muon channel (Run IIb). 

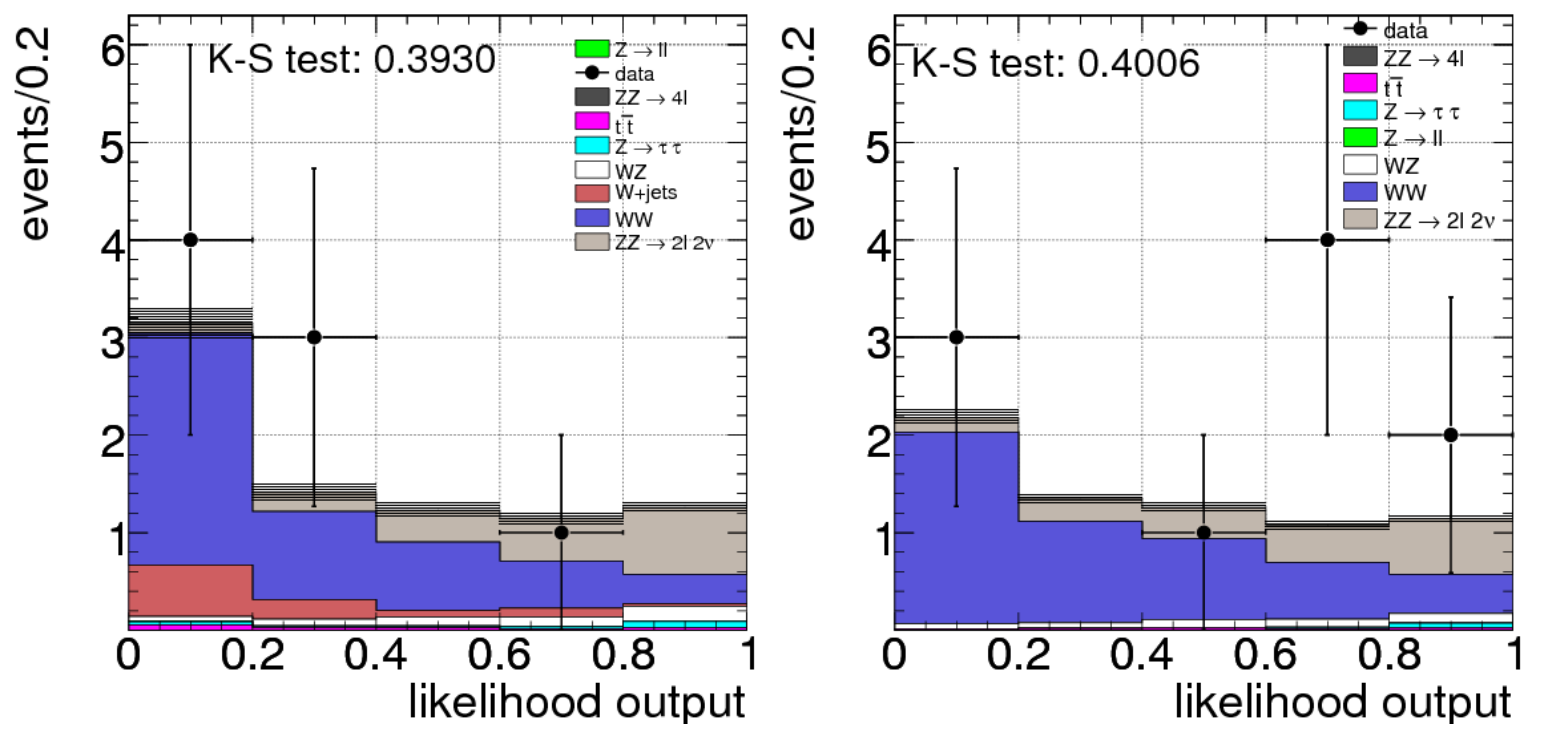

(a) RunIIa dataset
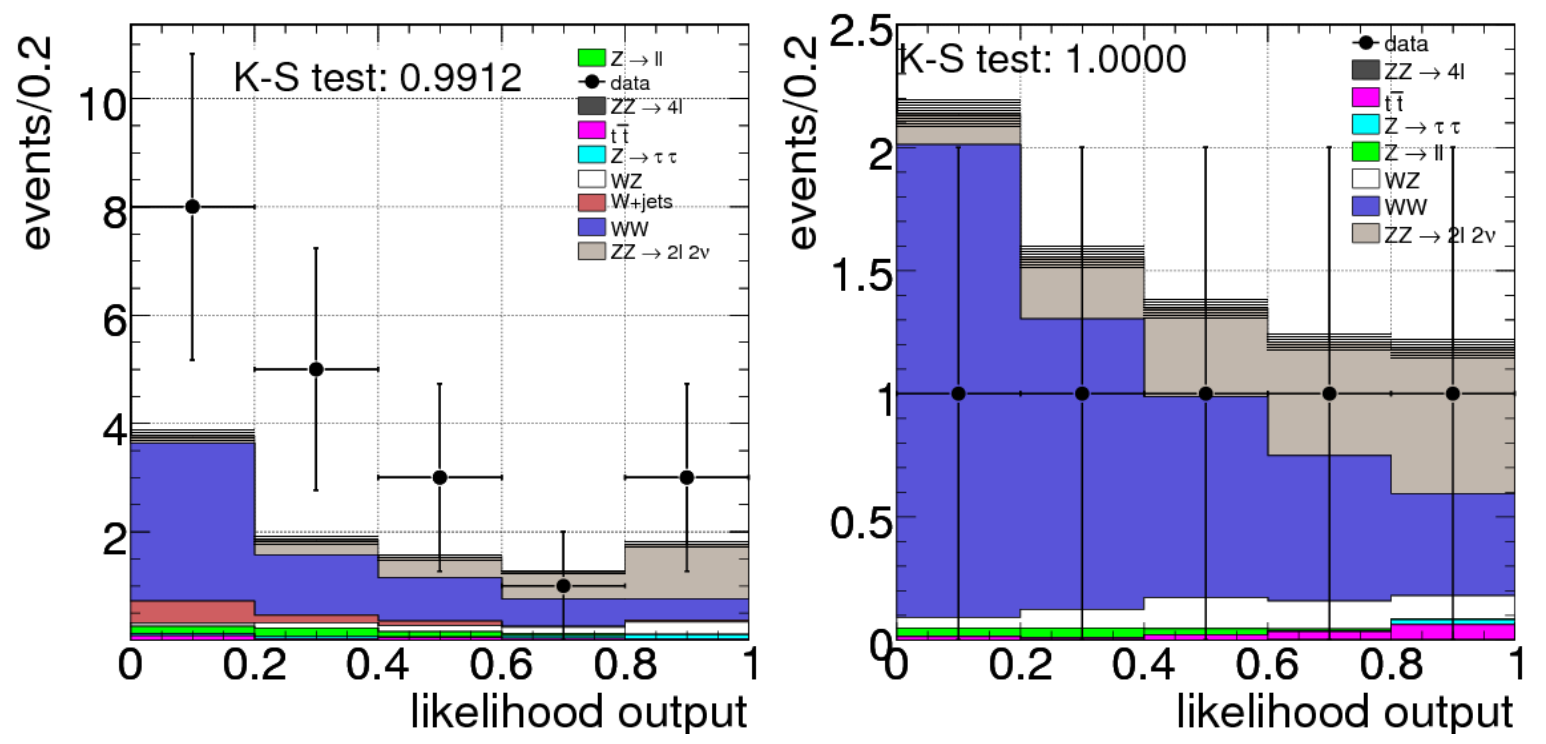

(b) RunIIb dataset

Figure 7.23: Output of the likelihood discriminant for the di-electron (left) and di-muon (right) final states. 


\subsection{Systematic Uncertainties}

In this section we will discuss the sources of error which may produce a systematic bias in our estimation of the signal and background efficiency or shape. These systematic uncertainties are treated as nuisance parameters in the limit calculation method described in Chapter 6 .

\subsubsection{Significance Systematics}

The systematic uncertainties which affect the calculation of the sensitivity are described below.

Jet Energy Scale (JES) The JES is found to only affect the normalization of the samples since none of the likelihood variables depend on it. The uncertainty is determined by fluctuating the JES up or down by one sigma. Since the effect is not always symmetric, the largest positive variation after the $\pm 1 \sigma$ shift is used as the positive scale uncertainty, and vice versa for the negative scale uncertainty. In the case where the shifts produce same signed variations, only the largest of the two is used.

Jet Energy Resolution (JER) The JER is treated identically to the JES by varying the MC smearing by $\pm 1 \sigma$.

Jet Reconstruction Efficiency (Jet Eff) The Jet Eff uncertainty is estimated by repeating the analysis, shifting the jet reconstruction parametrization down by one $\sigma$.

Lepton Resolution The smearing of the lepton energies to match the detector resolution is parametrized as a function of $p_{T}$ and $\eta$ for the electrons and muons. The parameters of the function are varied up or down one $\sigma$ to assess the size of the lepton resolution systematic. Since no Run IIa electron smearing was available at the time of this analysis, the study was performed only on Run IIb samples and assumed to apply equally to Run IIa. The change in shape is propagated as a shape uncertainty.

$W+$ jets Normalization The estimation of the $W+$ jets yield in our sample relies on a set of variables (real lepton selection rate, faked lepton selection rate, number of "loose" and "tight" events) each ov which have an associated error. The error is propagated through the matrix method formula as inputs to our significance calculation. Due to the lack of statistics in the sample after the $\mathbb{E}_{T}^{\prime}$ cut, the uncertainties are quite large, which has a substantial impact on the sensitivity of this study.

$\mathscr{E}_{T}^{\prime}$ Modeling The yields after the $\not_{T}^{\prime}$ cut were estimated using three different methods. The first two are determined by fitting the falling edge of the variable to an exponential and then integrating the function above the cutoff for the Data and the MC. The third is calculated by simply summing the predicted MC events which are in bins above the cut. 
Table 7.10: Uncertainties on the $W+$ jets normalization.

\begin{tabular}{|lcc|}
\hline \hline \multicolumn{3}{c|}{ Run IIa } \\
\hline Channel & Normalization & Relative uncertainty \\
\hline di-electron & $1.64 \pm 0.3$ & $30 \%$ \\
di-muon & $0.03 \pm 0.05$ & $166 \%$ \\
\hline \multicolumn{3}{|c|}{ Run IIb } \\
\hline di-electron & $1.08 \pm 0.3$ & $28 \%$ \\
di-muon & $-0.02 \pm 0.02$ & $100 \%$ \\
\hline \hline
\end{tabular}

The relevant curves are shown in Figures 7.24 and 7.25. The central value of the $\mathrm{Z}$ contribution is computed from the number of surviving $\mathrm{MC}$ events while the uncertainty on this number is estimated comparing the extrapolation of the $\mathrm{MC}$ and data fits.

Table 7.11: Number of Z events surviving the $\mathbb{E}_{T}^{\prime}$ cut as estimated from the MC and from the exponential fits to the falling edge of the $\mathscr{E}_{T}^{\prime}$ distribution in data and MC.

\begin{tabular}{|lcccc|}
\hline \hline \multicolumn{5}{c|}{ Run IIa } \\
\hline Channel & MC & Fit to MC & Fit to Data & Uncertainty \\
\hline di-electron & 0.04 & 0.07 & 0.07 & $6.7 \%$ \\
di-muon & 0.009 & 0.009 & 0.01 & $32 \%$ \\
\hline \multicolumn{5}{c}{ Run IIb } \\
\hline di-electron & 0.43 & 0.36 & 0.27 & $20 \%$ \\
di-muon & 0.11 & 0.009 & 0.007 & $2.0 \%$ \\
\hline \hline
\end{tabular}

$W W$ and $W Z$ Production Cross Sections The theoretical value of the $W W$ and $W Z$ cross sections has a $7 \%$ uncertainty [41] which is fed as a flat systematic to the significance and cross section calculations.

\subsubsection{Cross Section Systematics}

The calculation of the $Z Z$ cross section is additionally affected by the following uncertainties:

$Z / \gamma^{*}$ theoretical cross section The normalization of the MC to the $Z$ peak width makes this analysis insensitive to trigger efficiency systematics; instead the uncertainty on the $Z / \gamma^{*}$ theoretical cross section [41] must be propagated to the $Z Z$ cross section measurement.

$\frac{A_{Z}}{A_{Z Z}}$ acceptance ratio due to pdf uncertainties The $Z / \gamma^{*}$ and $Z Z$ production mechanisms probe different regions of the pdf distributions. Variations in the pdf distributions can affect the lepton pseudorapidity of the two processes in different ways. The corresponding 
uncertainty on the $\frac{A_{Z}}{A_{Z Z}} Z$ and $Z Z$ acceptance ratio used to compute the cross section was estimated using the CTEQ prescription. The events are re-weighted to the CTEQ6.1M pdf set and the ratio $\frac{A_{Z}}{A_{Z Z}}$ is computed. The ratio is also calculated for 20 sets of positive and negative variations of the pdf parameters. The difference of the ratio $\frac{A_{Z}}{A_{Z Z}}$ with respect to the central value are added in quadrature for each of the $20 \times 2$ variations. The resulting uncertainties are listed in Table 7.12 for the di-electron and di-muon final states. The conservative value of $1.8 \%$ is used in the estimate of the cross section uncertainties.

Table 7.12: pdf uncertainties on the $\frac{A_{Z}}{A_{Z Z}}$ ratio as estimated with the CTEQ prescription.

\begin{tabular}{|cccc|}
\hline \hline \multicolumn{2}{|c|}{ di-electron } & \multicolumn{2}{c|}{ di-muon } \\
\hline$+1.5 \%$ & $+1.8 \%$ & $-1.8 \%$ & $+1.7 \%$ \\
\hline \hline
\end{tabular}

$\frac{A_{Z}}{A_{Z Z}}$ ratio due to Modeling of the veto $\mathrm{A}$ systematic uncertainty is assigned to the ratio $\frac{A_{Z}}{A_{Z Z}}$ to account for the modeling of the veto efficiency in the MC. The size of the uncertainty is estimated to be $0.8 \%$ based on the measured difference of efficiency in data and MC.

$\frac{A_{Z}}{A_{Z Z}}$ ratio due to Modeling of the $Z p_{T}$ spectrum We apply a re-weighting function on the $Z Z p_{T}$, derived by fitting the $p_{T}$ spectra of $W W$ in Sherpa and PYTHiA. A 3.0\% uncertainty is estimated from the effect on the $Z Z$ acceptance. 


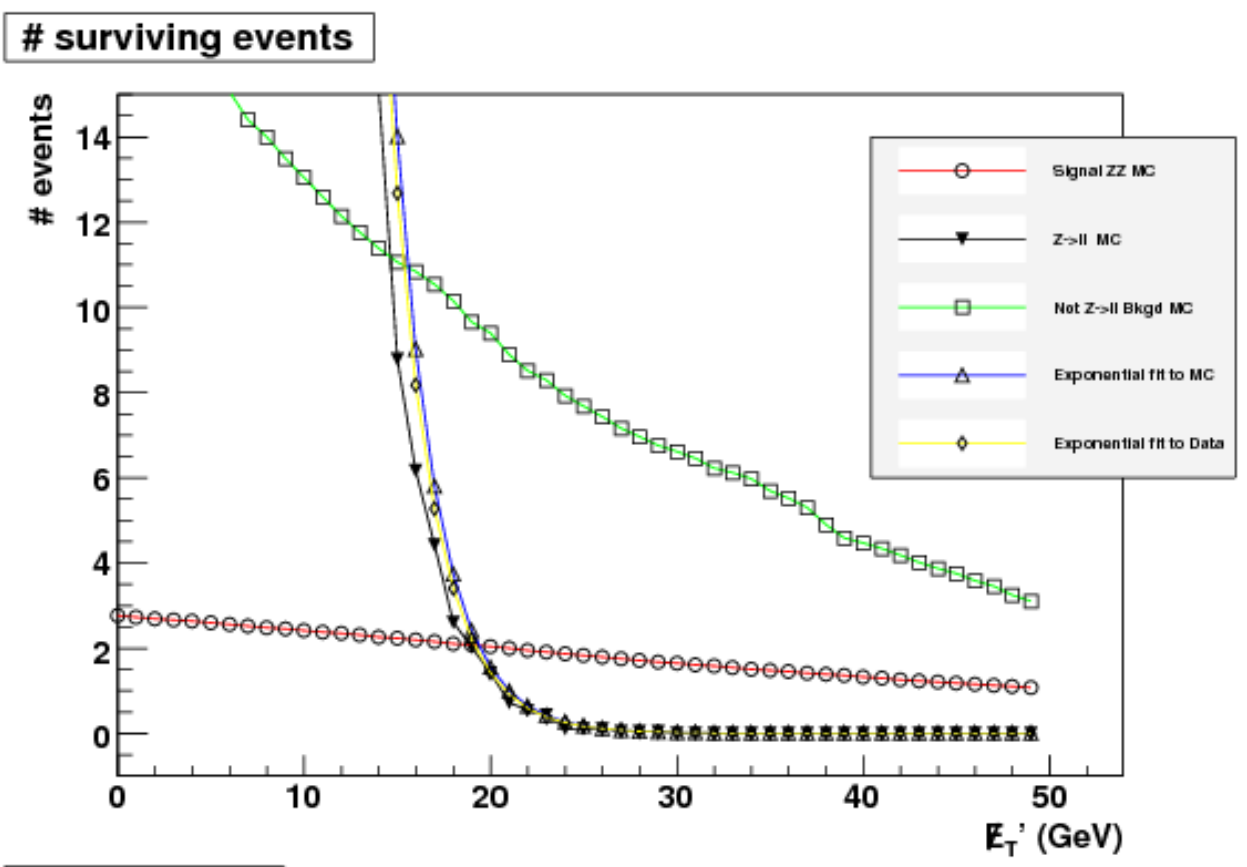

cut efficiency

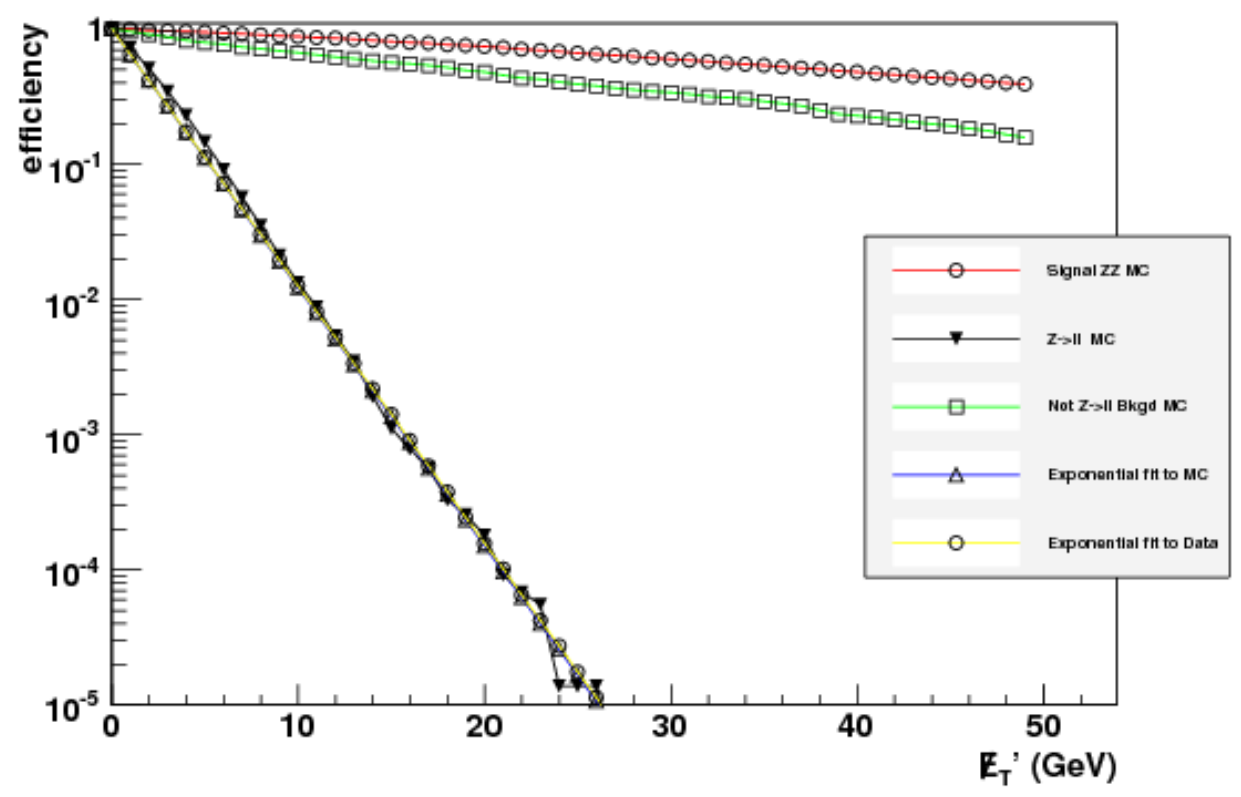

Figure 7.24: Run IIa: Number of events (top) and fraction remaining (bottom) varying the cut (along the $\mathrm{x}$ axis) on the $\mathbb{E}_{T}^{\prime}$ of the event in the di-electron channel. The red line results in summing events in the signal MC, the black line in summing the $\mathrm{Z} \rightarrow$ ee $\mathrm{MC}$, and the green line in summing all the other background MC. The blue and yellow lines are obtained by integrating an exponential which has been fit to the falling edge in the $\mathrm{MC}$ and Data respectively. 


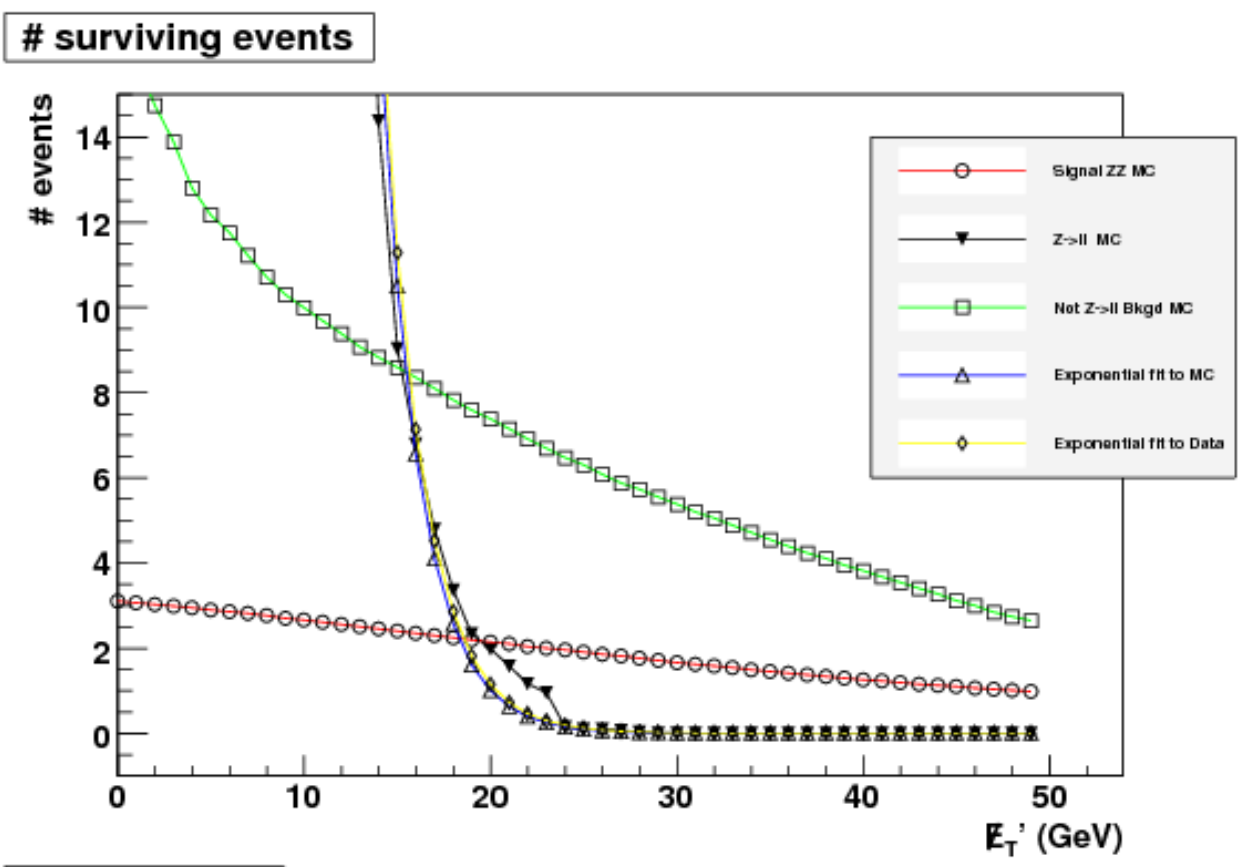

cut efficiency

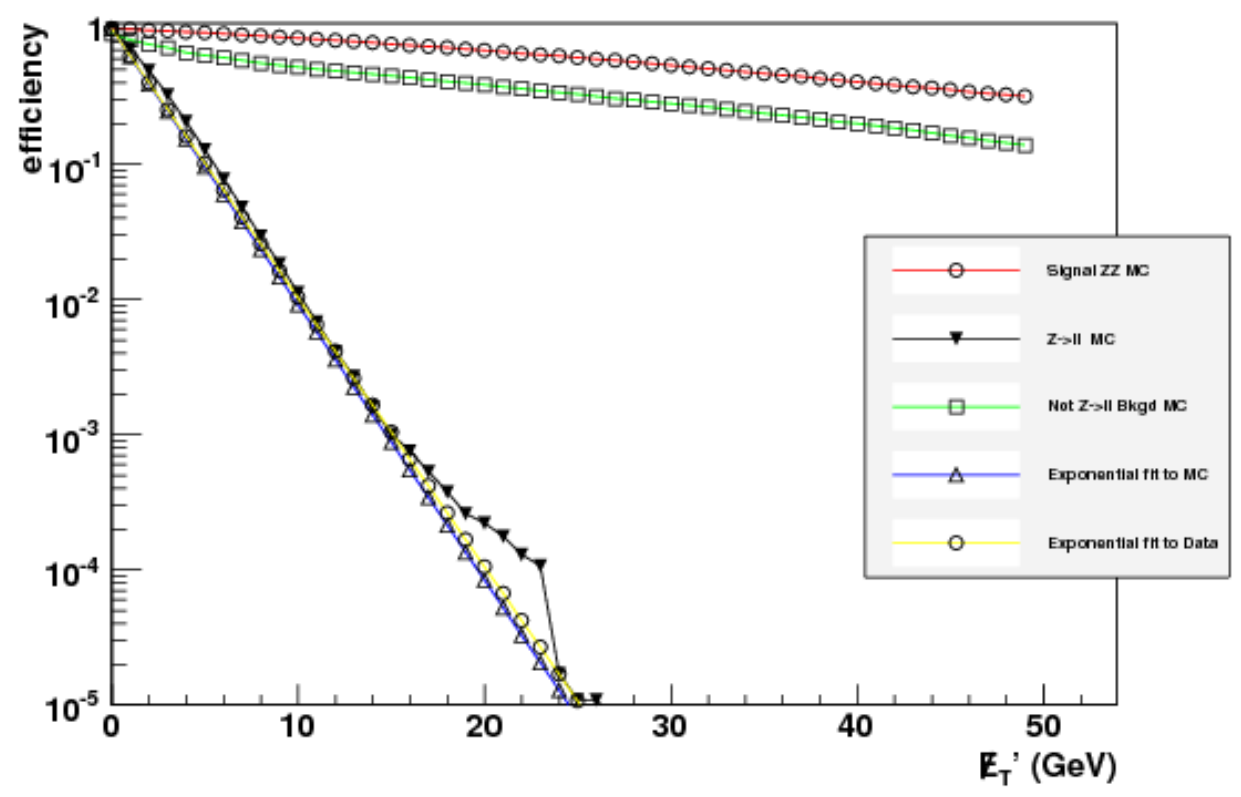

Figure 7.25: Run IIa: Number of events (top) and fraction remaining (bottom) varying the cut (along the $\mathrm{x}$ axis) on the $\mathbb{E}_{T}^{\prime}$ of the event in the di-muon channel. The red line results in summing events in the signal MC, the black line in summing the $\mathrm{Z} \rightarrow \mu \mu \mathrm{MC}$, and the green line in summing all the other background MC. The blue and yellow lines are obtained by integrating an exponential which has been fit to the falling edge in the $\mathrm{MC}$ and Data respectively. 


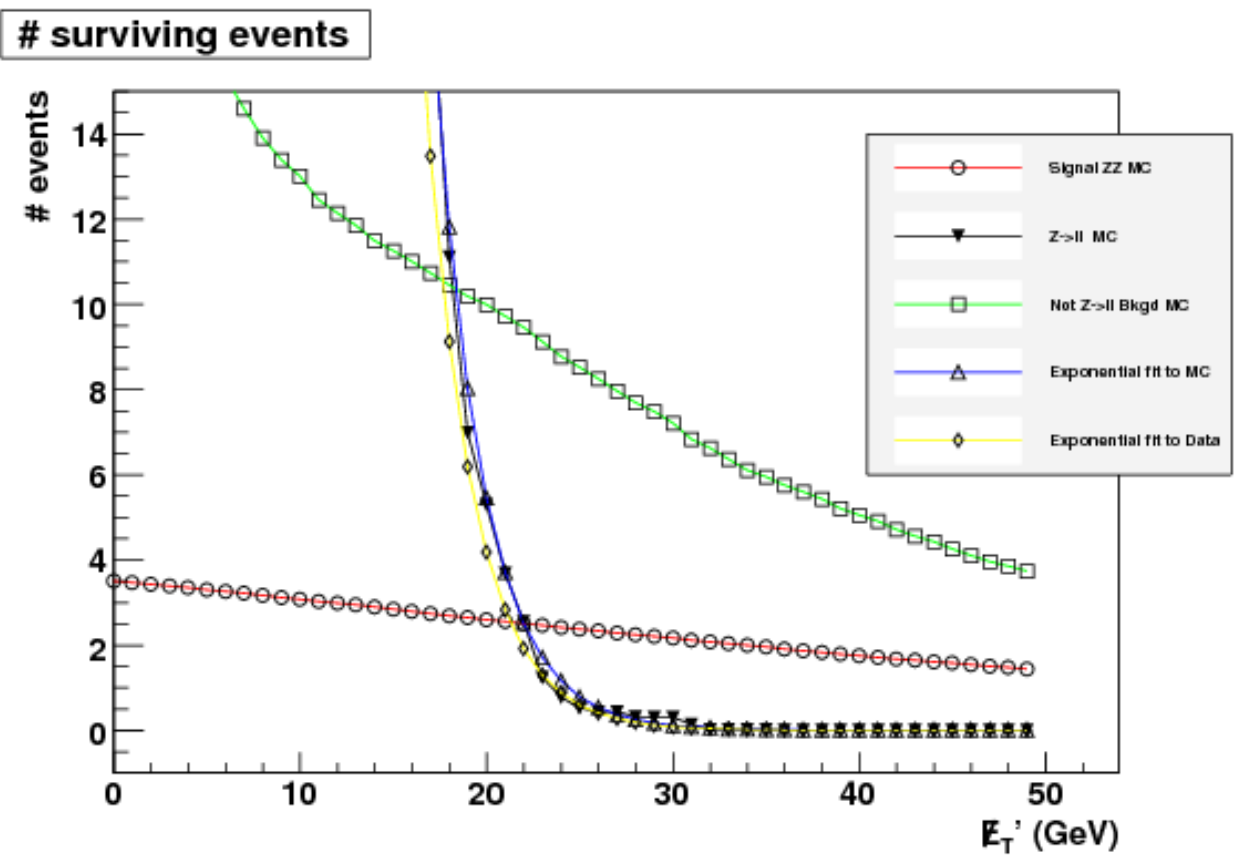

cut efficiency

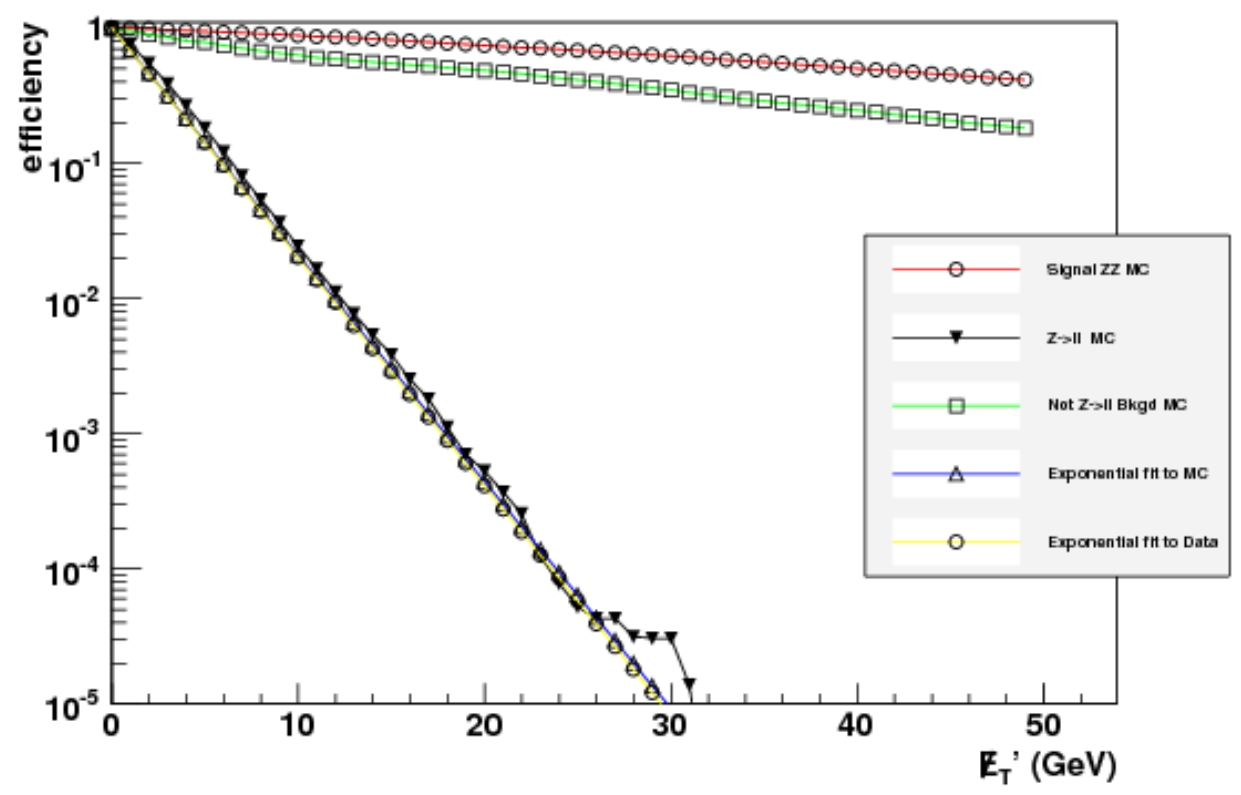

Figure 7.26: Run IIb: Number of events (top) and fraction remaining (bottom) varying the cut (along the x axis) on the $\mathbb{E}_{T}^{\prime}$ of the event in the di-electron channel. The red line results in summing events in the signal $\mathrm{MC}$, the black line in summing the $\mathrm{Z} \rightarrow \mathrm{ee} \mathrm{MC}$, and the green line in summing all the other background MC. The blue and yellow lines are obtained by integrating an exponential which has been fit to the falling edge in the $\mathrm{MC}$ and Data respectively. 


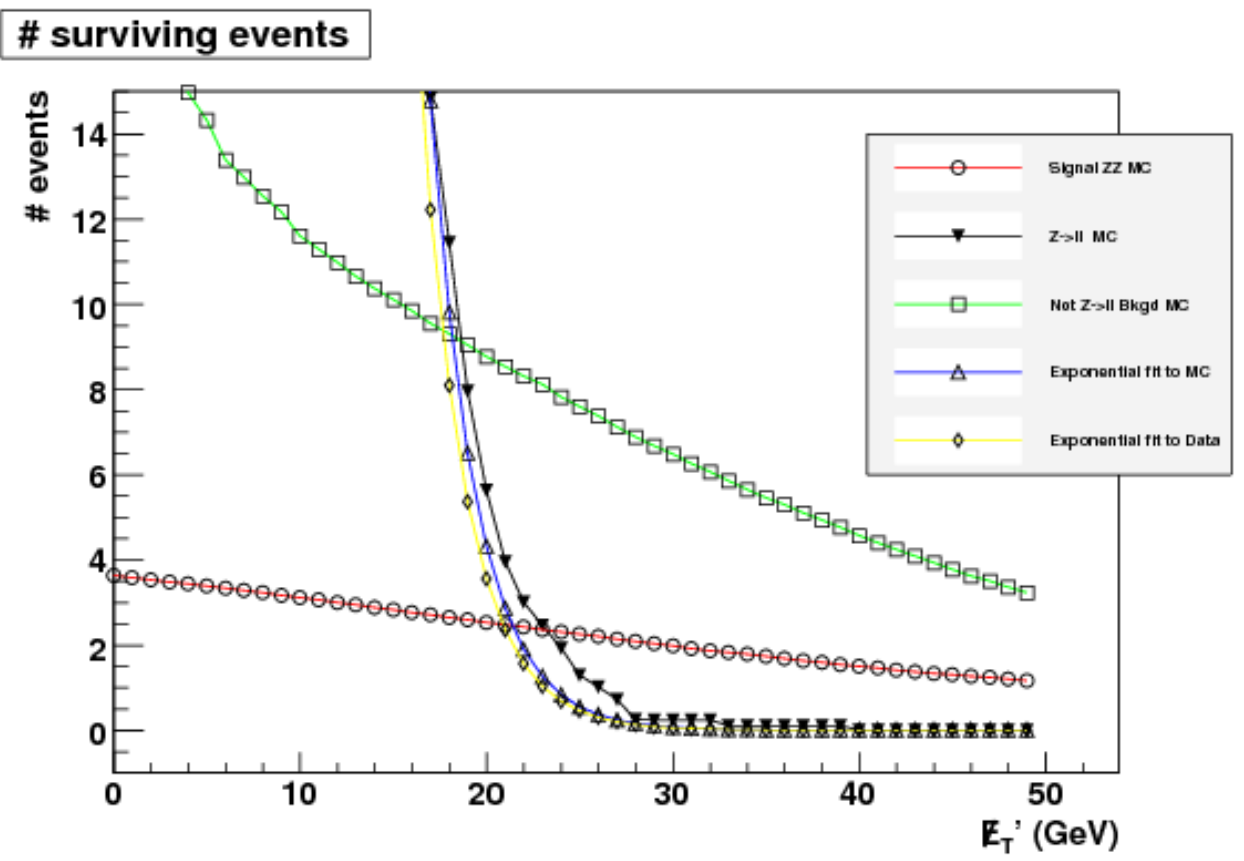

cut efficiency

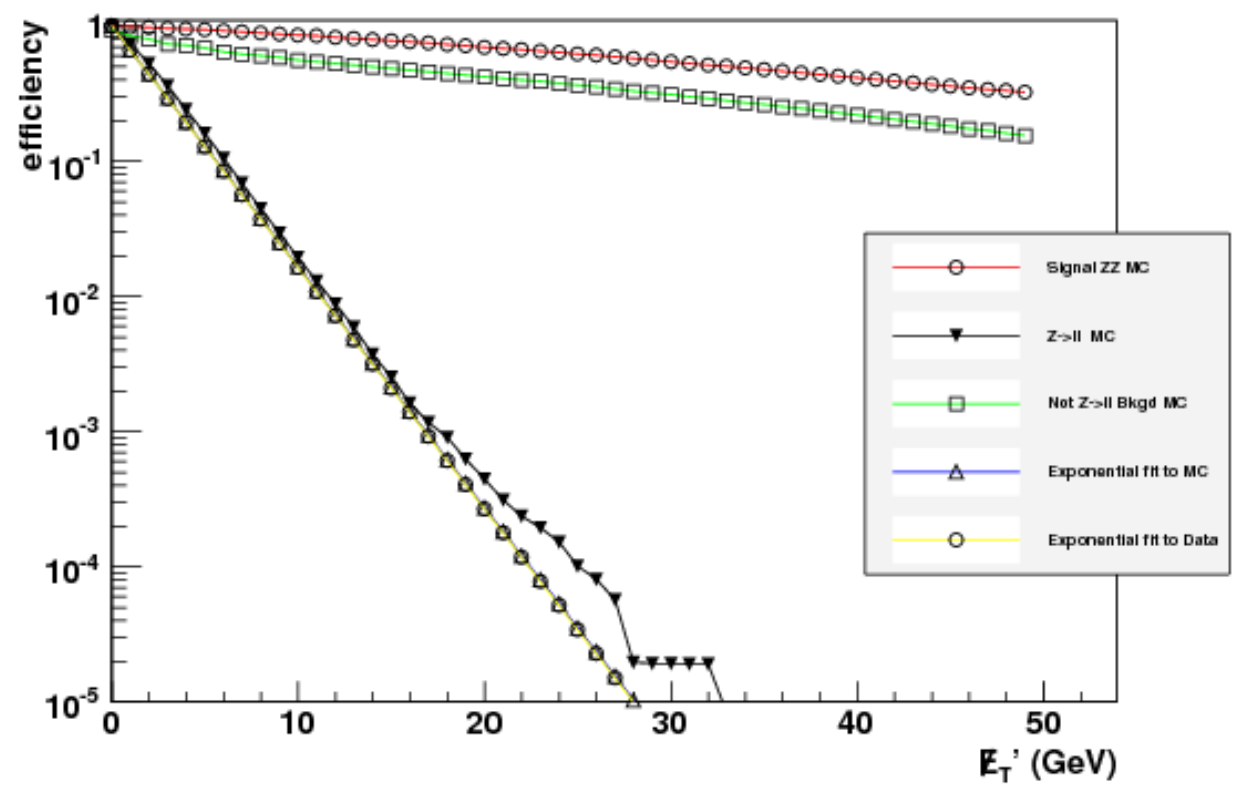

Figure 7.27: Run IIb: Number of events (top) and fraction remaining (bottom) varying the cut (along the $\mathrm{x}$ axis) on the $\mathscr{E}_{T}^{\prime}$ of the event in the di-muon channel. The red line results in summing events in the signal $\mathrm{MC}$, the black line in summing the $\mathrm{Z} \rightarrow \mu \mu \mathrm{MC}$, and the green line in summing all the other background MC. The blue and yellow lines are obtained by integrating an exponential which has been fit to the falling edge in the $\mathrm{MC}$ and Data respectively. 


\subsection{Cross Section Measurement and Significance}

The $Z Z$ cross section measurement is performed on the binned output of the likelihood variable (See Section 7.6). The systematics described in Section 7.7 are considered as nuisance parameters in the fits to the data and pseudo-experiments.

The plots in Figure 7.28 show the distribution of the LLR test statistics computed by COLLIE as a result of 200,000 pseudo-experiments for the signal plus background hypothesis and for the background only hypothesis. The plots also report the median of the signal plus background distribution (red vertical line) which can be considered as the expected value for the test statistics. The value actually observed is represented by the black vertical line.

The values of $C L_{b}, p$-value, and the expected (observed) significance in the di-electron and di-muon channels are reported in Table 7.13 for Run IIa and Run IIb data.

An independent search for $Z Z$ production where both $Z$ bosons decay to charged leptons has also been performed at $\mathrm{D} \varnothing$ [34]. The $Z Z \rightarrow \ell \ell \nu \nu$ and $Z Z \rightarrow \ell^{+} \ell^{-} \ell^{\prime+} \ell^{\prime-}$ final states and the two sets of data are finally combined and a global significance is then estimated. The resulting LLR distributions are shown in Figures 7.28, 7.29, and 7.30. We observe (expect) a signal with $+5.69 \sigma(+4.77 \sigma)$ significance, as reported in the last column of Table 7.13 , and measure a cross section $\sigma(p \bar{p} \rightarrow Z Z)=1.60 \pm 0.63$ (stat.) ${ }_{-0.17}^{+0.16}$ (sys.) pb. This is in agreement with the theoretical cross section of $1.4 \pm 0.1 \mathrm{pb}[41]$.

Table 7.13: Estimated (Observed) significance value using CoLLIE for the di-electron, di-muon, and four charged leptons channels as well as their combination. Systematics have been included in the computation, but not profiling. Since no events were observed in the Run IIa $Z Z \rightarrow$ $\ell^{+} \ell^{-} \ell^{\prime+} \ell^{\prime-}$ analysis, the observed $C L_{b}$ and significance values are 0 .

\begin{tabular}{|c|c|c|c|c|}
\hline & $\begin{array}{c}Z Z \rightarrow e^{+} e^{-} \nu \bar{\nu} \\
\text { expected (observed) }\end{array}$ & $\begin{array}{c}Z Z \rightarrow \mu^{+} \mu^{-} \nu \bar{\nu} \\
\text { expected (observed) }\end{array}$ & $\begin{array}{c}Z Z \rightarrow \ell^{+} \ell^{-} \ell^{\prime+} \ell^{\prime-} \\
\text { expected (observed) }\end{array}$ & $\begin{array}{c}\text { Combined } \\
\text { expected (observed) }\end{array}$ \\
\hline \multicolumn{5}{|c|}{ Run IIa } \\
\hline$C L_{b}$ & $0.860(0.421)$ & $0.834(0.992)$ & $0.988(0.00)$ & $0.998(0.891)$ \\
\hline $\mathrm{p}$-value & $0.1401(0.579)$ & $0.166(0.00830)$ & $0.0119(1.00)$ & $0.00162(0.109)$ \\
\hline significance & $1.08(-0.20) \sigma$ & $0.970(2.40) \sigma$ & $2.26(0.00) \sigma$ & $2.94(1.23) \sigma$ \\
\hline \multicolumn{5}{|c|}{ Run IIb } \\
\hline$C L_{b}$ & $0.892(0.997)$ & $0.845(0.678)$ & $0.999(1.00)$ & $0.999(1.00)$ \\
\hline $\mathrm{p}$-value & $0.108(0.00350)$ & $0.155(0.322)$ & $4.18 \times 10^{-4}\left(4.26 \times 10^{-8}\right)$ & $9.48 \times 10^{-5}\left(2.18 \times 10^{-9}\right)$ \\
\hline significance & $1.24(2.70) \sigma$ & $1.01(0.46) \sigma$ & $3.34(5.36) \sigma$ & $3.73(5.87) \sigma$ \\
\hline \multicolumn{5}{|c|}{ combined } \\
\hline$C L_{b}$ & $0.938(0.981)$ & $0.907(0.978)$ & $0.999(1.00)$ & $0.999(1.00)$ \\
\hline $\mathrm{p}$-value & $0.0616(0.0195)$ & $0.0933(0.0222)$ & $7.26 \times 10^{-6}\left(2.88 \times 10^{-7}\right)$ & $9.08 \times 10^{-7}\left(6.22 \times 10^{-9}\right)$ \\
\hline significance & $1.54(2.06) \sigma$ & $1.32(2.01) \sigma$ & $4.34(5.00) \sigma$ & $4.77(5.69) \sigma$ \\
\hline
\end{tabular}



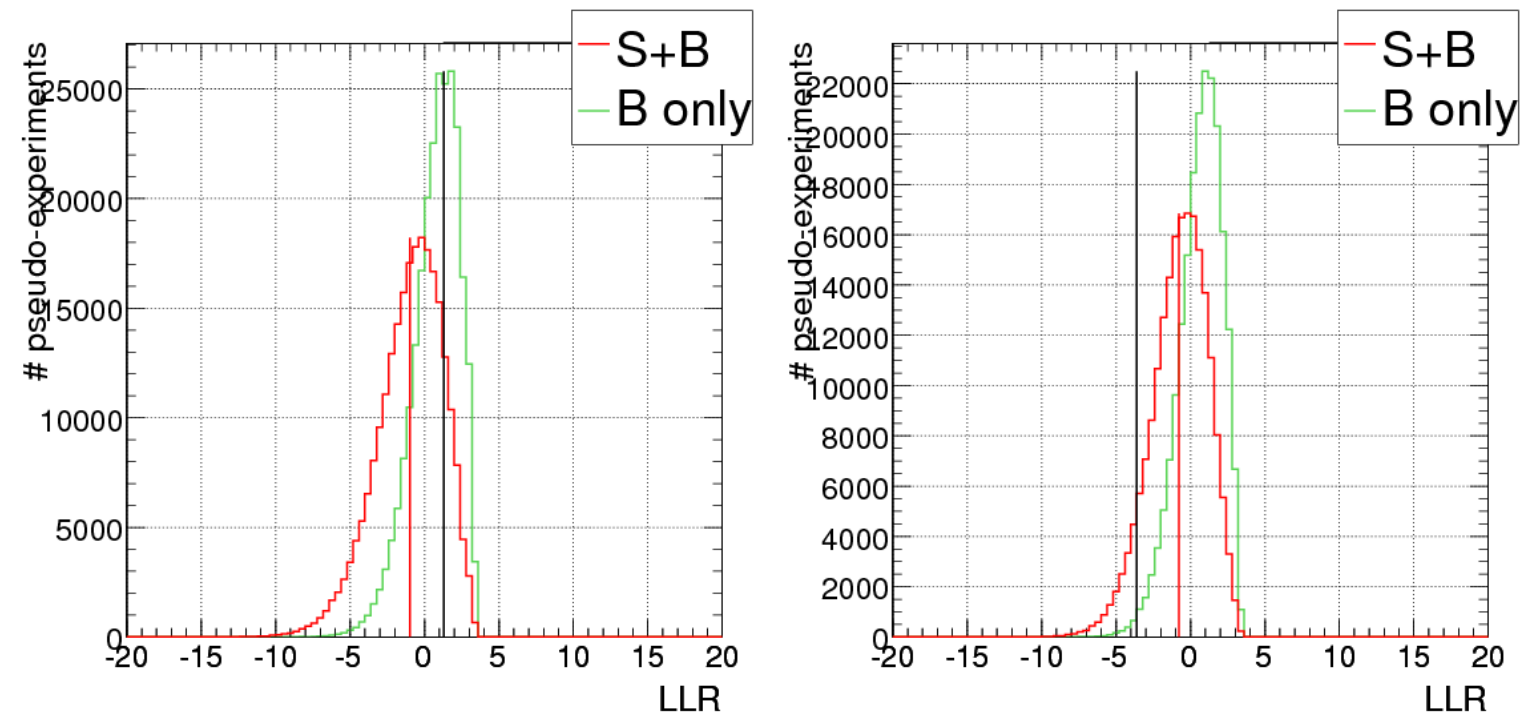

(a) Run IIa
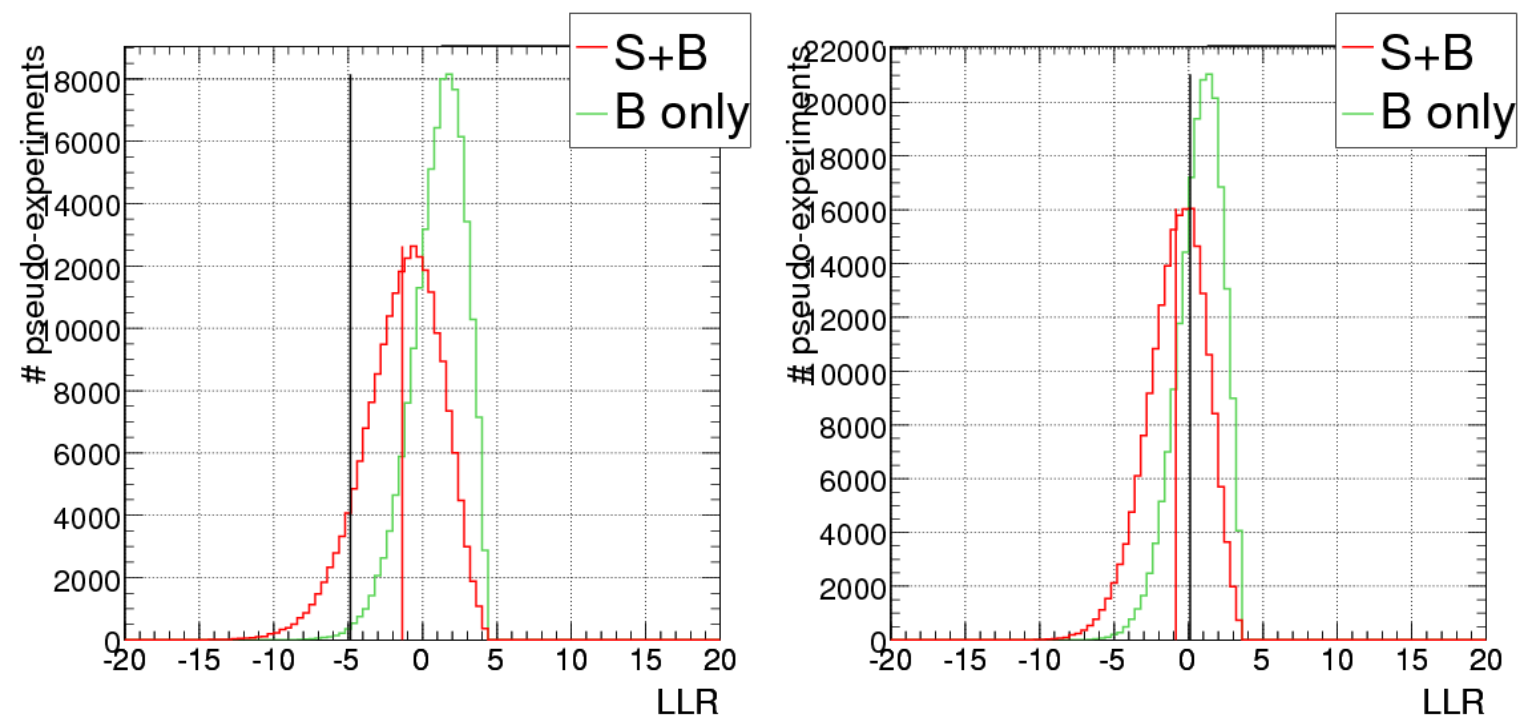

(b) Run IIb

Figure 7.28: LLR distribution computed by CoLLIE in several pseudo-experiments for the signal plus backgrounds $(\mathrm{S}+\mathrm{B})$ and background only (B only) hypothesis in the di-electron(left) and di-muon (right) channels using Run IIa and Run IIb datasets. The vertical red line indicates the median of the $\mathrm{S}+\mathrm{B}$ distribution while the vertical black line indicates the actual value measured in the data. 


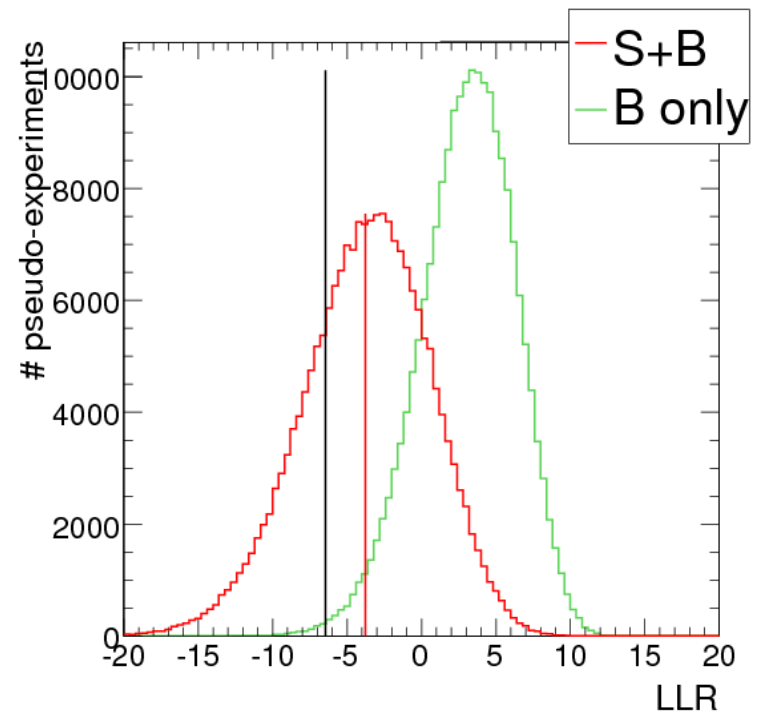

Figure 7.29: LLR distribution computed by COLLIE in several pseudo-experiments for the signal plus backgrounds $(\mathrm{S}+\mathrm{B})$ and background only (B only) hypothesis combining the di-electron and di-muon final states for the Run IIa and Run IIb datasets. The vertical red line indicates the median of the $\mathrm{S}+\mathrm{B}$ distribution while the vertical black line indicates the actual value measured in the data. 


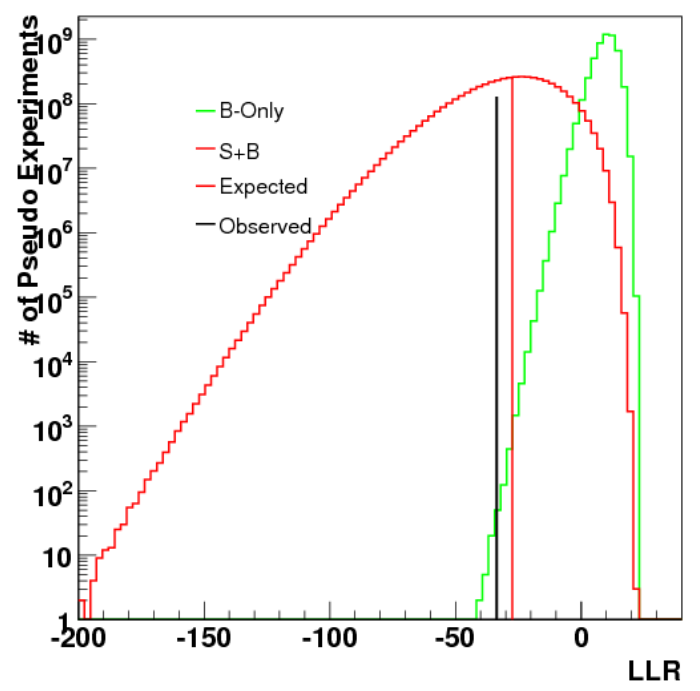

Figure 7.30: LLR distribution computed by COLLIE in several pseudo-experiments for the signal plus backgrounds $(\mathrm{S}+\mathrm{B})$ and background only (B only) hypothesis combining the di-electron, di-muon, and four charged leptons final states for the Run IIa and Run IIb datasets. The vertical red line indicates the median of the $\mathrm{S}+\mathrm{B}$ distribution while the vertical black line indicates the actual value measured in the data. 


\section{Chapter 8}

\section{$Z H \rightarrow \ell \ell b b$ Analysis}

"Arrêtez-vous Lagaffe! On ne voit rien!" "Ben! On n'y verra pas plus si on s'arrête, malin!"

Although the existence of the Higgs Boson was postulated over 40 years ago as part of the mechanism which explains the mass of the weak force mediating bosons [42], it has yet to be experimentally observed. Higgs events are exceedingly rare, if they occur at all, and until the LHC begins operations, the Tevatron at Fermilab remains the most likely place for first observation of the Higgs boson.

A direct result of the additional terms in the Electroweak Lagrangian (See Section 1.2.3), the coupling of the Higgs boson to fermions $(H f \bar{f})$ grows linearly with the fermion mass, whereas the $H W W(H Z Z)$ coupling is proportional to $M_{W}^{2}\left(M_{Z}^{2}\right)$. The resulting decay modes, with respect to the Higgs mass are shown in Figure 8.1. Since the most probable Higgs decay mode is to the heaviest possible final state, a low mass Higgs $\left(m_{H}<135 \mathrm{GeV}\right)$ decays dominantly to two $b$-quarks. Gluon mixing is the dominant Higgs production mechanism at Hadron colliders, but this $g g \rightarrow H \rightarrow b \bar{b}$ mode unfortunately suffers from a large amount of multijet background. In this low mass Higgs regime, we must rely on associated production of the Higgs with a $W$ or $Z$ boson. The leptonic decay of the $W$ or $Z$ provides an experimental signature we can use to reduce the background. Theoretical constraints from Electroweak measurements indicate a strong preference for a low mass Higgs $\left(m_{H}=90_{-27}^{+36} \mathrm{GeV}\right.$ at $68 \%$ confidence level and $m_{H}<163 \mathrm{GeV}$ at $95 \%$ confidence level [43]), whereas direct searches at LEP have excluded it below $114.4 \mathrm{GeV}$. As shown in Figure 8.1, the cross section of associated production of a Higgs and $Z$ or $H$ is an order of magnitude smaller than from gluon fusion. Although our sensitivity to a low mass Higgs is reduced, it is the most likely place for us to be searching.

In this chapter we describe a search for $Z H$ production in the decay channel where the $H$ decays to two b quarks, and the $Z$ decays to two muons. The traditional muonic coverage 

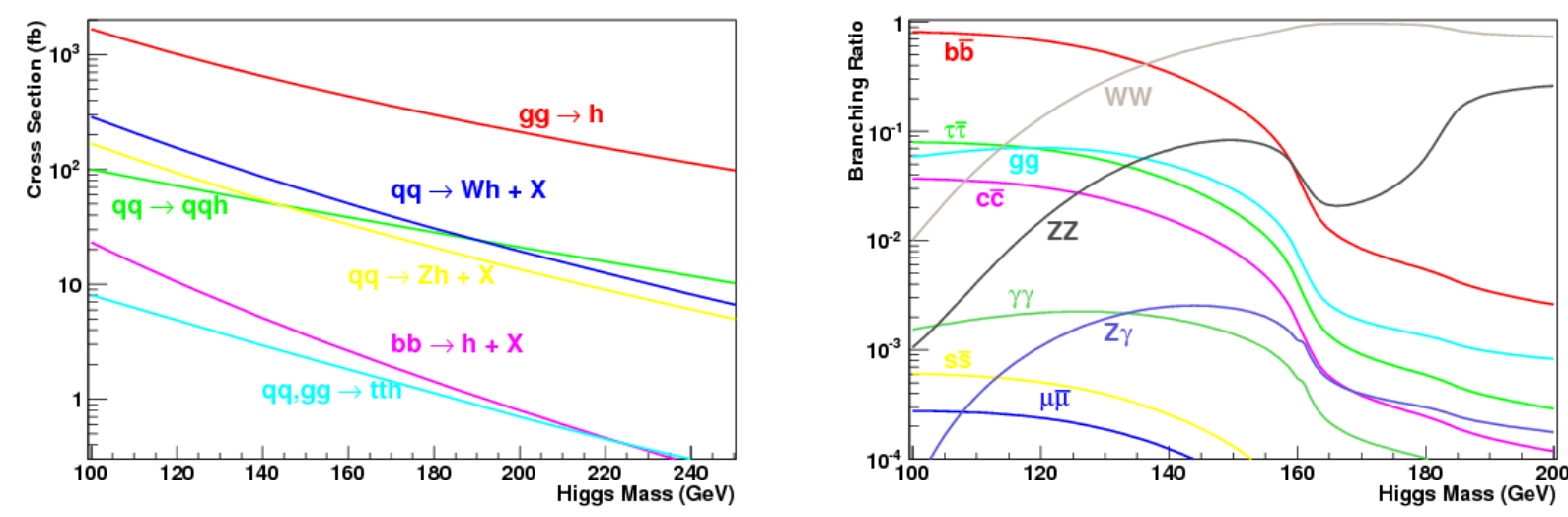

Figure 8.1: Higgs Boson production (left) and decays (right) at the Tevatron as a function of the Higgs-boson mass [44].

has been extended by considering events in which only one of the muons pass the selection requirements. We do not differentiate between cases where the second muon was not reconstructed at all (for example, by passing through reduced coverage regions of the detector) and cases where it was reconstructed but failed analysis level quality cuts. These lost muons are recovered by considering a category of tightly isolated tracks where the muon and track invariant mass fall within our $Z$ mass window. The rate of lost muons has been estimated to be about $20 \%$. Through the use of these track-only muons, we increase the $Z$ event yield by $15 \%$, recovering a large fraction of these muons.

The sensitivity of this Higgs search was further improved by developing a kinematic fit which improves the jet energy resolution. The sharper di-jet mass resonant peak is of benefit to this, as well as the three other complimentary $Z H \rightarrow \ell \ell b b$ searches in which the fit was used.

Since no individual search has the required sensitivity, four $Z H \rightarrow \ell \ell b b$ searches are developed jointly. They share common $b$-jet tagging requirements, use the same multivariate technique to discriminate between signal and backgrounds, and the $95 \%$ confidence limits on the Higgs cross section is determined through their combination.

\subsection{Dataset and Monte Carlo Samples}

\subsubsection{Data}

The data for this analysis were collected at the D0 detector between April 2002 and September 2008 at the Fermilab Tevatron collider at $p \bar{p}$ center-of-mass energy $\sqrt{s}=1.96 \mathrm{TeV}$. The integrated luminosity before data quality requirements is $4.3 \mathrm{fb}^{-1}$. Data quality information is used to remove runs and lbns marked as bad in the calorimeter, muon, cft, and smt systems. 
Event quality flags are used to further improve the quality of our data sample. After all quality driven cuts, the luminosity is $3.6 \mathrm{fb}^{-1}$. Although this luminosity has been determined using the JT_125TT and JT125_L3J125 un-prescaled triggers, no explicit trigger requirement is made when selecting the data. The data is selected from the Common Samples Group skims. This sample is comprised of three logical skims:

- SKIM_2MU1TRK where two Loose muons have been reconstructed, one with $p_{T}>$ $15.0 \mathrm{GeV}$.

- SKIM_2MUhighpt where where two Loose muons have been reconstructed, each with $p_{T}>10.0 \mathrm{GeV}$.

- SKIM_MU2TRKhighpt where one Loose muon has been reconstructed, matched to a $>15.0 \mathrm{GeV}$ track and there is at least one other $>15.0 \mathrm{GeV}$ track in the event.

It it the third of these logical skims that we rely on for our events.

Unlike the $Z Z$ analysis, the multijet background is entirely modeled from data by selecting a sample in which the muon and track have the same charge. To cope with the small statistics

in this sub-set of the data, the multijet background after b-tagging is modeled by re-normalizing the un-b-tagged sampled to the Data in the low di-lepton invariant mass range $\left(40<M_{\ell \ell}<\right.$ $70 \mathrm{GeV})$.

\subsubsection{Monte Carlo}

The signal and all other backgrounds are modeled by MC produced using a mix of PYTHIA and AlPGEN+PythiA samples (See Table 8.1 and Table 8.2). In each case we set the relative scale between the MC samples using their Next-to-Leading-Order (NLO) cross sections. The $Z / \gamma^{*} \mathrm{MC}$ are split into three samples, those with heavy flavor content $(Z+b b$ and $Z+c c)$, and those with light partons $(Z+l p)$.

\subsubsection{Additional Monte Carlo Corrections}

The following corrections are applied to the $Z H$ analysis in addition to those described in Section 3.3.

- Heavy Flavor Skimming : Instead of modeling the inclusive $Z$ sample with PYTHIA, the $\overline{Z H}$ analysis uses ALPGEN+PYTHIA MC in which the samples have been generated in bins of heavy and light parton multiplicity. However, AlPGEN treats $c$-quarks as light partons, and the fragmentation modeled by PYTHIA includes decays to $b$-quarks. To avoid double counting, we apply a Heavy Flavor skim to each sample using the parton information to ensure that the $Z+q \bar{q}$ sample contains only light jets, the $Z+c \bar{c}$ sample contains only $c$-jets, and the $Z+b \bar{b}$ sample contains only $b$-jets. 


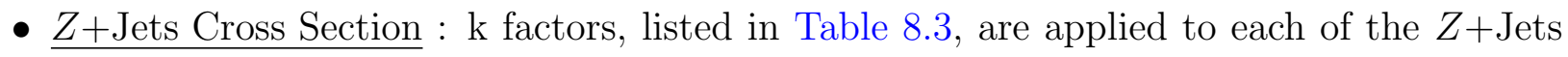
samples. The common factor of 1.30 scales the AlPGEN Leading Log (LL) cross sections to those measured in data. A second $\mathrm{k}$ factor, calculated using MCFC, is applied to account for differences in the LO and NLO calculations for the $Z+b \bar{b}$ and $Z+c \bar{c}$ in Alpgen $[45,46]$.

- Alpgen Jet Angles: The Alpgen modeling of the jet $\eta$ distributions do not agree with the data. A common set of ALPGEN Vector Boson plus Jets ( $V+$ Jets) corrections have been derived on samples selected by the $Z H \rightarrow \ell \ell b b, H \rightarrow W W \rightarrow \ell \nu j j$, and $W H \rightarrow \ell \nu b b$ analyses. The non-ALPGEN MC is subtracted from the data and a re-weighting factor is fit using a $4^{\text {th }}$ order polynomial for the leading jet $\eta$, second jet $\eta, \Delta \phi(j, j), \Delta \eta(j, j)$, and $\Delta R(j, j)$ distributions [47].

- Vertex Confirmed Jets : The high-luminosity environment of the Run IIb dataset makes it substantially more likely to have minimum bias jets misidentified as coming from the $\mathrm{PV}$. An additional scale factor is applied to the $\mathrm{MC}$ as a function of the $\mathrm{PV} z$ position and the sign of $\eta$ [48]. The scale factors are parametrized using a second order polynomial determined in $\eta$ bins of 0.4 .

- Jet Taggability: The fraction of taggable jets is not the same in Data and MC, nor is the size of the discrepancy the same for Run IIa and Run IIb. Scale factors, parametrized as two dimensional functions of jet $\eta$ and $p_{T}$, are used to randomly set jets in $\mathrm{MC}$ as non-taggable $[49,50]$.

- Inclusive Triggering : Although no explicit trigger requirement is made in this analysis, we must still correct for the trigger acceptance effects which are not modeled by the MC. Rather than re-derive the trigger corrections covered in Section 3.3 for this inclusive case, we select the data with a trigger suite for which corrections already exist. The ratio of this explicitely triggered dataset to the inclusive dataset provides us with an over-correction we can apply to the MC. This method is further detailed in Appendix D. 
Table 8.1: p17 Signal and Background Monte Carlo Samples

\begin{tabular}{|c|c|c|c|c|}
\hline $\begin{array}{l}\text { Process } \\
\end{array}$ & $\begin{array}{l}\text { Mass Range }(\mathrm{GeV}) \\
\end{array}$ & 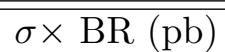 & Generator & Events \\
\hline$\overline{Z Z / \gamma^{*}+0 l p \rightarrow \mu \mu}$ & $15<M(Z)<75$ & 338.363 & " ALPGEN+PYTHIA & 2551685 \\
\hline$Z / \gamma^{*}+0 l p \rightarrow \mu \mu$ & $75<M(Z)<130$ & 133.103 & AlPGEN+Pythia & 2834535 \\
\hline$Z / \gamma^{*}+0 l p \rightarrow \mu \mu$ & $130<M(Z)<250$ & 1.335 & AlPGEN+Pythia & 462867 \\
\hline$Z / \gamma^{*}+1 l p \rightarrow \mu \mu$ & $15<M(Z)<75$ & 39.942 & AlPGEN+Pythia & 460472 \\
\hline$Z / \gamma^{*}+1 l p \rightarrow \mu \mu$ & $75<M(Z)<130$ & 40.677 & AlPGEN+Pythia & 1938896 \\
\hline$Z / \gamma^{*}+1 l p \rightarrow \mu \mu$ & $130<M(Z)<250$ & 0.931 & AlPGEN+Pythia & 374280 \\
\hline$Z / \gamma^{*}+2 l p \rightarrow \mu \mu$ & $15<M(Z)<75$ & 9.870 & ALPGEN+Pythia & 183369 \\
\hline$Z / \gamma^{*}+2 l p \rightarrow \mu \mu$ & $75<M(Z)<130$ & 9.7514 & ALPGEN+PythIA & 1017484 \\
\hline$Z / \gamma^{*}+2 l p \rightarrow \mu \mu$ & $130<M(Z)<250$ & 0.480 & ALPGEN+Pythia & 284171 \\
\hline$Z / \gamma^{*}+3 l p \rightarrow \mu \mu$ & $15<M(Z)<75$ & 2.739 & ALPGEN+PYTHIA & 91069 \\
\hline$Z / \gamma^{*}+3 l p \rightarrow \mu \mu$ & $75<M(Z)<130$ & 3.1148 & ALPGEN+Pythia & 1054299 \\
\hline$Z / \gamma^{*}+3 l p \rightarrow \mu \mu$ & $130<M(Z)<250$ & 0.053 & ALPGEN+PYTHIA & 286302 \\
\hline$Z / \gamma^{*}+2 b+0 l p \rightarrow \mu \mu$ & $75<M(Z)<130$ & 0.4175 & ALPGEN+Pythia & 185534 \\
\hline$Z / \gamma^{*}+2 b+1 l p \rightarrow \mu \mu$ & $75<M(Z)<130$ & 0.1904 & ALPGEN+Pythia & 94233 \\
\hline$Z / \gamma^{*}+2 b+2 l p \rightarrow \mu \mu$ & $75<M(Z)<130$ & 0.09859 & AlPGEN+Pythia & 47998 \\
\hline$Z / \gamma^{*}+2 c+0 l p \rightarrow \mu \mu$ & $75<M(Z)<130$ & 0.4175 & AlPGEN+Pythia & 185203 \\
\hline$Z / \gamma^{*}+2 c+1 l p \rightarrow \mu \mu$ & $75<M(Z)<130$ & 0.1904 & ALPGEN+Pythia & 96462 \\
\hline$Z / \gamma^{*}+2 c+2 l p \rightarrow \mu \mu$ & $75<M(Z)<130$ & 0.09859 & ALPGEN+Pythia & 48217 \\
\hline$Z Z \rightarrow 2 j 2 \ell$ & & 0.226 & PYTHIA & 563589 \\
\hline$W Z \rightarrow 2 j 2 \mu$ & & 0.092 & PYTHIA & 94128 \\
\hline$W W \rightarrow$ incl & & 12.35 & PYTHIA & 4690024 \\
\hline$t t \rightarrow 2 b 2 \ell 2 \nu$ & & 0.67 & PYTHIA & 633152 \\
\hline$Z H \rightarrow \mu \mu b b$ & $M(H)=100$ & 0.0137 & PYTHIA & 184362 \\
\hline$Z H \rightarrow \mu \mu b b$ & $M(H)=105$ & 0.0116 & Pyтhia & 183234 \\
\hline$Z H \rightarrow \mu \mu b b$ & $M(H)=110$ & 0.0096 & Pyтhia & 183009 \\
\hline$Z H \rightarrow \mu \mu b b$ & $M(H)=115$ & 0.00797 & PYTHIA & 184048 \\
\hline$Z H \rightarrow \mu \mu b b$ & $M(H)=120$ & 0.00635 & PYThIA & 183891 \\
\hline$Z H \rightarrow \mu \mu b b$ & $M(H)=125$ & 0.00501 & Рyтhia & 172518 \\
\hline$Z H \rightarrow \mu \mu b b$ & $M(H)=130$ & 0.00375 & PYTHIA & 183076 \\
\hline$Z H \rightarrow \mu \mu b b$ & $M(H)=135$ & 0.00274 & Рythia & 183669 \\
\hline$Z H \rightarrow \mu \mu b b$ & $M(H)=140$ & 0.00188 & Pythia & 172481 \\
\hline$Z H \rightarrow \mu \mu b b$ & $M(H)=145$ & 0.00123 & РYTHIA & 183469 \\
\hline$Z H \rightarrow \mu \mu b b$ & $M(H)=150$ & 0.00075 & Pythia & 183086 \\
\hline
\end{tabular}


Table 8.2: p20 Signal and Background Monte Carlo Samples

\begin{tabular}{|c|c|c|c|c|}
\hline Process & Mass Range $(\mathrm{GeV})$ & $\overline{\sigma \sigma \times \mathrm{BR}(\mathrm{pb})}$ & Generator & Events \\
\hline$\overline{Z Z / \gamma^{*}+0 l p \rightarrow \mu \mu}$ & $15<M(Z)<75$ & $\begin{array}{l}338.363 \\
\end{array}$ & "ALPGEN+PYTHIA & 1805548 \\
\hline$Z / \gamma^{*}+0 l p \rightarrow \mu \mu$ & $75<M(Z)<130$ & 133.103 & AlPGEN+Pythia & 1036678 \\
\hline$Z / \gamma^{*}+0 l p \rightarrow \mu \mu$ & $130<M(Z)<250$ & 1.335 & AlPGEN+Pythia & 271249 \\
\hline$Z / \gamma^{*}+1 l p \rightarrow \mu \mu$ & $15<M(Z)<75$ & 39.942 & AlPGEN+Pythia & 336634 \\
\hline$Z / \gamma^{*}+1 l p \rightarrow \mu \mu$ & $75<M(Z)<130$ & 40.677 & AlPGEN+Pythia & 616917 \\
\hline$Z / \gamma^{*}+1 l p \rightarrow \mu \mu$ & $130<M(Z)<250$ & 0.931 & ALPGEN+Pythia & 173777 \\
\hline$Z / \gamma^{*}+2 l p \rightarrow \mu \mu$ & $15<M(Z)<75$ & 9.870 & ALPGEN+PYTHIA & 197528 \\
\hline$Z / \gamma^{*}+2 l p \rightarrow \mu \mu$ & $75<M(Z)<130$ & 9.7514 & ALPGEN+PYTHIA & 432267 \\
\hline$Z / \gamma^{*}+2 l p \rightarrow \mu \mu$ & $130<M(Z)<250$ & 0.480 & ALPGEN+PYTHIA & 173118 \\
\hline$Z / \gamma^{*}+3 l p \rightarrow \mu \mu$ & $15<M(Z)<75$ & 2.739 & ALPGEN+PYTHIA & 317512 \\
\hline$Z / \gamma^{*}+3 l p \rightarrow \mu \mu$ & $75<M(Z)<130$ & 3.1148 & ALPGEN+Pythia & 166902 \\
\hline$Z / \gamma^{*}+3 l p \rightarrow \mu \mu$ & $130<M(Z)<250$ & 0.053 & ALPGEN+PYTHIA & 164316 \\
\hline$Z / \gamma^{*}+2 b+0 l p \rightarrow \mu \mu$ & $75<M(Z)<130$ & 0.4175 & AlPGEN+Pythia & 179558 \\
\hline$Z / \gamma^{*}+2 b+1 l p \rightarrow \mu \mu$ & $75<M(Z)<130$ & 0.1904 & ALPGEN+Pythia & 96941 \\
\hline$Z / \gamma^{*}+2 b+2 l p \rightarrow \mu \mu$ & $75<M(Z)<130$ & 0.09859 & ALPGEN+Pythia & 47925 \\
\hline$Z / \gamma^{*}+2 c+0 l p \rightarrow \mu \mu$ & $75<M(Z)<130$ & 0.4175 & ALPGEN+Pythia & 187636 \\
\hline$Z / \gamma^{*}+2 c+1 l p \rightarrow \mu \mu$ & $75<M(Z)<130$ & 0.1904 & ALPGEN+PYTHIA & 89559 \\
\hline$Z / \gamma^{*}+2 c+2 l p \rightarrow \mu \mu$ & $75<M(Z)<130$ & 0.09859 & ALPGEN+PYTHIA & 49608 \\
\hline$Z Z \rightarrow 2 j 2 \ell$ & & 0.226 & РYThia & 105325 \\
\hline$W Z \rightarrow 2 j 2 \ell$ & & 0.275 & PYTHIA & 273344 \\
\hline$W W \rightarrow$ incl & & 12.35 & PYTHIA & 675814 \\
\hline$t t \rightarrow 2 b 2 \ell 2 \nu$ & & 0.67 & РҮTHIA & 257574 \\
\hline$Z H \rightarrow \mu \mu b b$ & $M(H)=100$ & 0.0137 & PYTHIA & 308876 \\
\hline$Z H \rightarrow \mu \mu b b$ & $M(H)=105$ & 0.0116 & PYTHIA & 270059 \\
\hline$Z H \rightarrow \mu \mu b b$ & $M(H)=110$ & 0.0096 & РYTHIA & 309248 \\
\hline$Z H \rightarrow \mu \mu b b$ & $M(H)=115$ & 0.00797 & PYTHIA & 270624 \\
\hline$Z H \rightarrow \mu \mu b b$ & $M(H)=120$ & 0.00635 & РҮTHIA & 311331 \\
\hline$Z H \rightarrow \mu \mu b b$ & $M(H)=125$ & 0.00501 & РҮTHIA & 270488 \\
\hline$Z H \rightarrow \mu \mu b b$ & $M(H)=130$ & 0.00375 & РҮTHIA & 311184 \\
\hline$Z H \rightarrow \mu \mu b b$ & $M(H)=135$ & 0.00274 & РутHIA & 270588 \\
\hline$Z H \rightarrow \mu \mu b b$ & $M(H)=140$ & 0.00188 & РҮтнIA & 309076 \\
\hline$Z H \rightarrow \mu \mu b b$ & $M(H)=145$ & 0.00123 & PYTHIA & 270064 \\
\hline$Z H \rightarrow \mu \mu b b$ & $M(H)=150$ & 0.00075 & РутHIA & 306021 \\
\hline
\end{tabular}

Table 8.3: Heavy Flavor k-Factors

\begin{tabular}{|c|ccc|}
\hline \hline & $Z l p$ & $Z c c$ & $Z b b$ \\
\hline k-factor & 1.30 & $1.30 * 1.67$ & $1.30 * 1.52$ \\
\hline \hline
\end{tabular}




\subsection{Preselection Requirements}

No explicit trigger requirement is made on the Data, all selection pairing is done on the event objects. The selection requirements described in this chapter were optimized given that we expect our $Z H$ events to contain two muons and two or more jets.

\subsubsection{Muon Selection}

Standard muon selection requirements are applied. We require one and only one muon reconstructed from scintillator and wire hits with the following requirements:

- $p_{T}>15 \mathrm{GeV}$

- Loose muon ID

- Matched central track with:

- A distance of closest approach of $<0.2 \mathrm{~cm}$ for track with no SMT hits, or

- A distance of closest approach of $<0.02 \mathrm{~cm}$ for track with at least one SMT hit

- Detector $\eta<2.0$

- $\left(\Sigma^{\text {cells }} E_{T}+\Sigma^{\text {tracks }} p_{T}\right) /\left(p_{T}(\right.$ track - only $\mu)<0.3$

\subsubsection{Track-only Muon Selection}

The purpose of this analysis is to extend the muon coverage by considering track-only muons which would have otherwise failed the standard muon selection requirements. As seen in Figure 8.2, the $Z$ invariant mass peak is visible in data even when pairing a muon with a track which satisfies only the loosest of isolation requirements imposed by the TMBIsoTrack branch (See Section 7.2.4).

The selection requirements are determined by varying the cuts on four million events of Run IIb data and optimizing on the mass peak in the $70<M_{\ell \ell}<130 \mathrm{GeV}$ window. The effectiveness of these cuts is assessed in the low mass tail below $40 \mathrm{GeV}$ which is dominated by background. Although the cuts were revisited in MC, this data driven round of optimization was found to be sufficient.

\section{Isolation}

In the first step, we vary the scaled isolation of the muon, track-only muon, as well as the product of the two as shown in Figure 8.3. The track halo and calorimeter isolation is not stored for individual tracks, so we must compute these values for ourselves from the tracks and calorimeter cells stored in the event. Since there is a $5 \mathrm{GeV}$ cut off for tracks included in the 


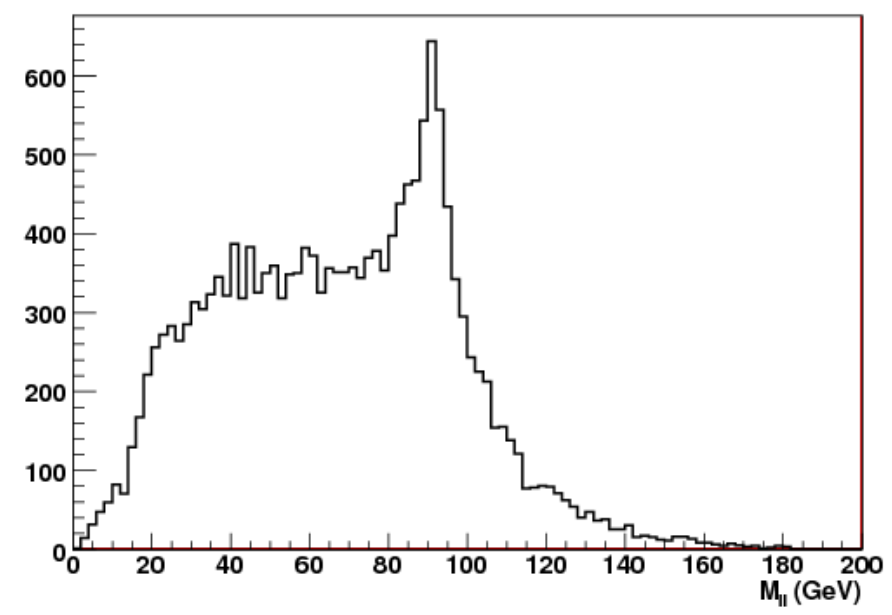

Figure 8.2: Data events containing one loose muon and a track which satisfies the TMBIsoTrack isolation requirements.

CAFE root files, the track-only muon's isolation definitions are not equivalent to that of the muon and as a result, direct cuts on the track-only muon are less effective.

\section{SMT Hits and DCA}

In the second step, we consider cuts on the DCA and number of SMT hits on the track. Figure 8.4 demonstrates that tracks with no SMT hits are entirely dominated by background. These SMT-less tracks have a uniform distribution with respect to their invariant mass, even at the central value of the $M_{\ell \ell}$ mass peak. We therefore discard any track which does not have at least one SMT hit and a DCA $<0.02 \mathrm{~cm}$.

\section{Charge}

By this point, the $M_{\ell \ell}$ distribution exhibits a clear peak around the $Z$ mass. Our third step applies a cut on the charge of the di-muon pair and is most visible in the low mass tail. Figure 8.5 shows that the same-sign sample's mass distribution is relatively uniform, indicating that it too is dominated by background.

\section{Transverse Momentum}

The fourth step varies the muon and track-only muon's $p_{T}$ from 15 to $25 \mathrm{GeV}$. Although raising the $p_{T}$ threshold does not have a large impact on the $M_{\ell \ell}$ distribution, our QCD and MC samples indicate that events with track-only muon $p_{T}<20 \mathrm{GeV}$ are dominated by background. 

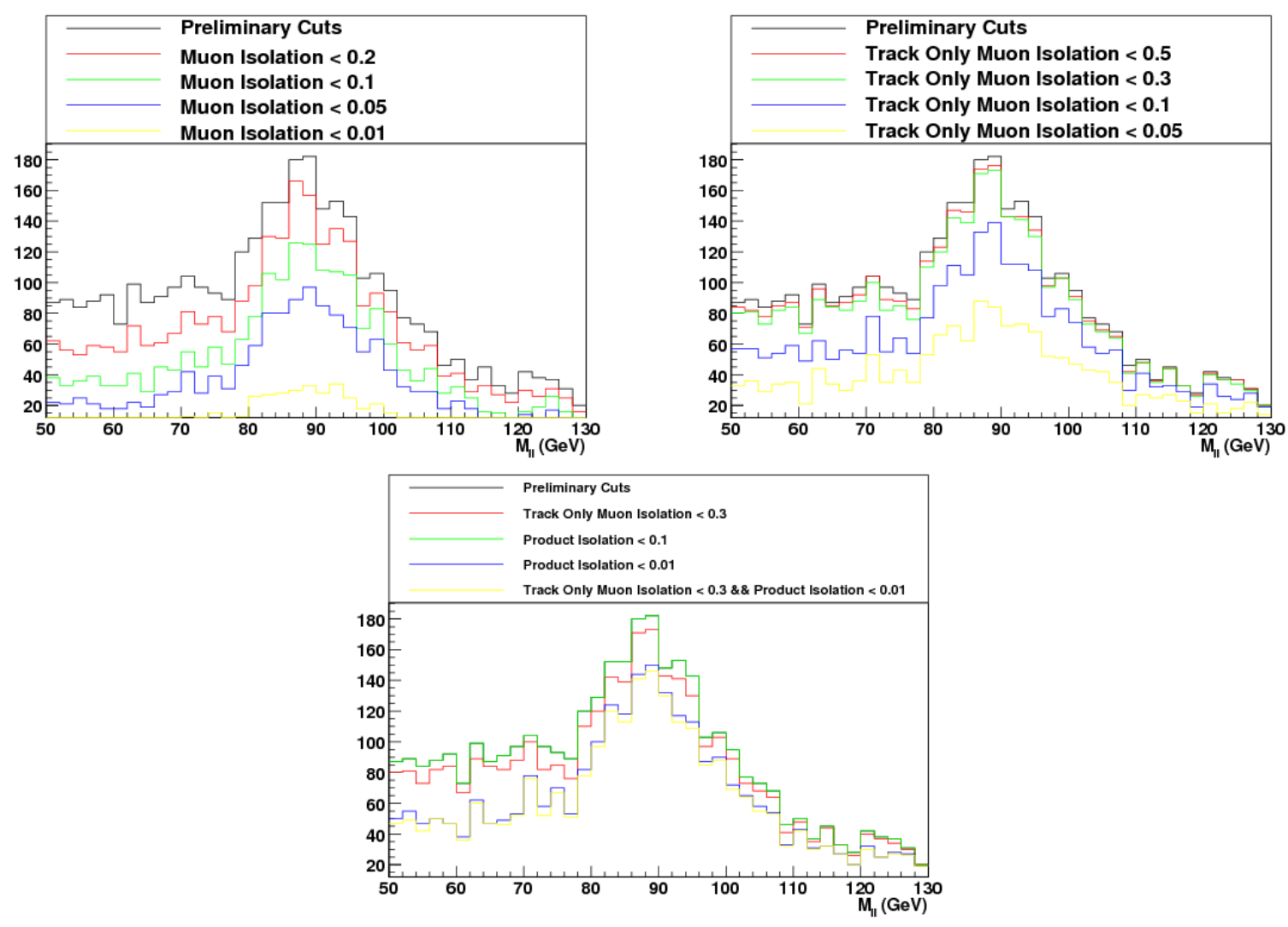

Figure 8.3: Di-lepton invariant mass, varying the isolation of the muon (top left), track only muon (top right), and their product (bottom center). The "Preliminary Cuts" distribution refers to the data sample with all the other cuts applied.

\section{Summarized Track Selection Requirements}

Our final selection requirements for the track-only muon are as follows:

- $p_{T}>20 \mathrm{GeV}$

- At least one SMT hit

- A distance of closest approach of $<0.02 \mathrm{~cm}$

- $\Delta R($ track - only $\mu, \mu)>0.1$

- $\Delta R($ track - only $\mu$, jet $)>0.5$

- Detector $\eta<2.0$

- $\mid\left(\Sigma^{\text {cells }} E_{T}+\Sigma^{\text {tracks }} p_{T}\right) /\left(p_{T}(\right.$ track - only $\left.\mu)\right)<0.3$ 


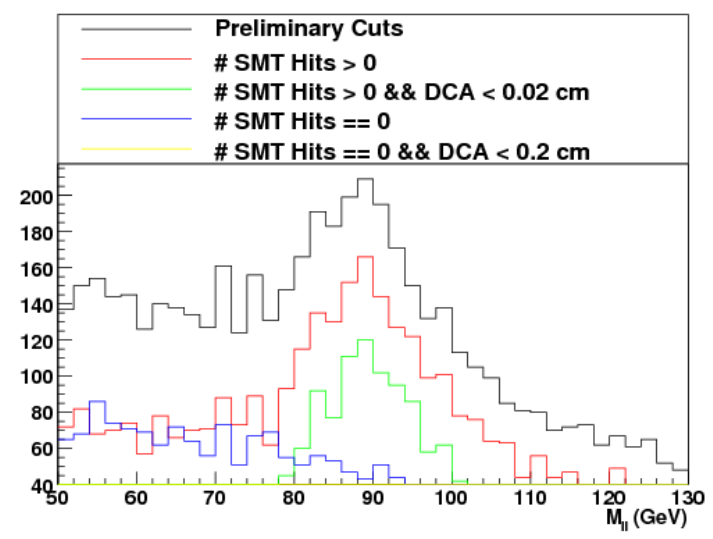

Figure 8.4: Di-lepton invariant mass, varying the cuts on the SMT hits and DCA of the track only muon.

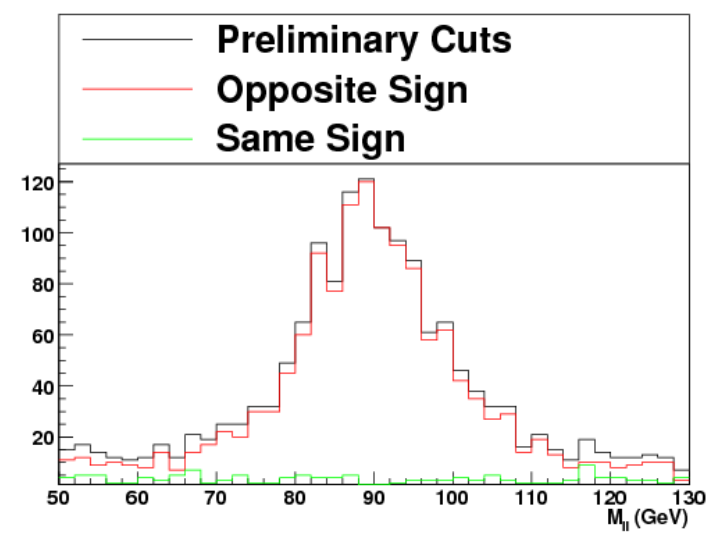

Figure 8.5: Di-lepton invariant mass, varying the cuts on charge ratio of the muon and track only muon. The "Preliminary Cuts" distribution refers to the data sample with all the other cuts applied.

The $\mu$ and track-only $\mu$ are required to have opposite charge, and they must be close in $z$ such that $\Delta z($ track - only $\mu, \mu)<1.0 \mathrm{~cm}$. Additionally, the scaled isolation product of the $\mu$ and track-only $\mu$ must be less than 0.01 :

$$
\prod_{i}\left(\Sigma_{i}^{\text {cells }} E_{T}+\Sigma_{i}^{\text {tracks }} p_{T}\right) /\left(p_{T}\left(\mu_{i}\right)\right)<0.01
$$

The invariant mass of the di-muon pair must be $70<M_{\ell \ell}<130 \mathrm{GeV}$.

Figure 8.7 shows the $\phi$ distribution of the muons in the traditional $Z H \rightarrow \mu \mu b b$ analysis and of the track-only muons in the $Z H \rightarrow \mu+\operatorname{trk} b b$ analysis. This clearly demonstrates how the muons lost in the bottom hole and in the octant gaps can be recovered using track-only 

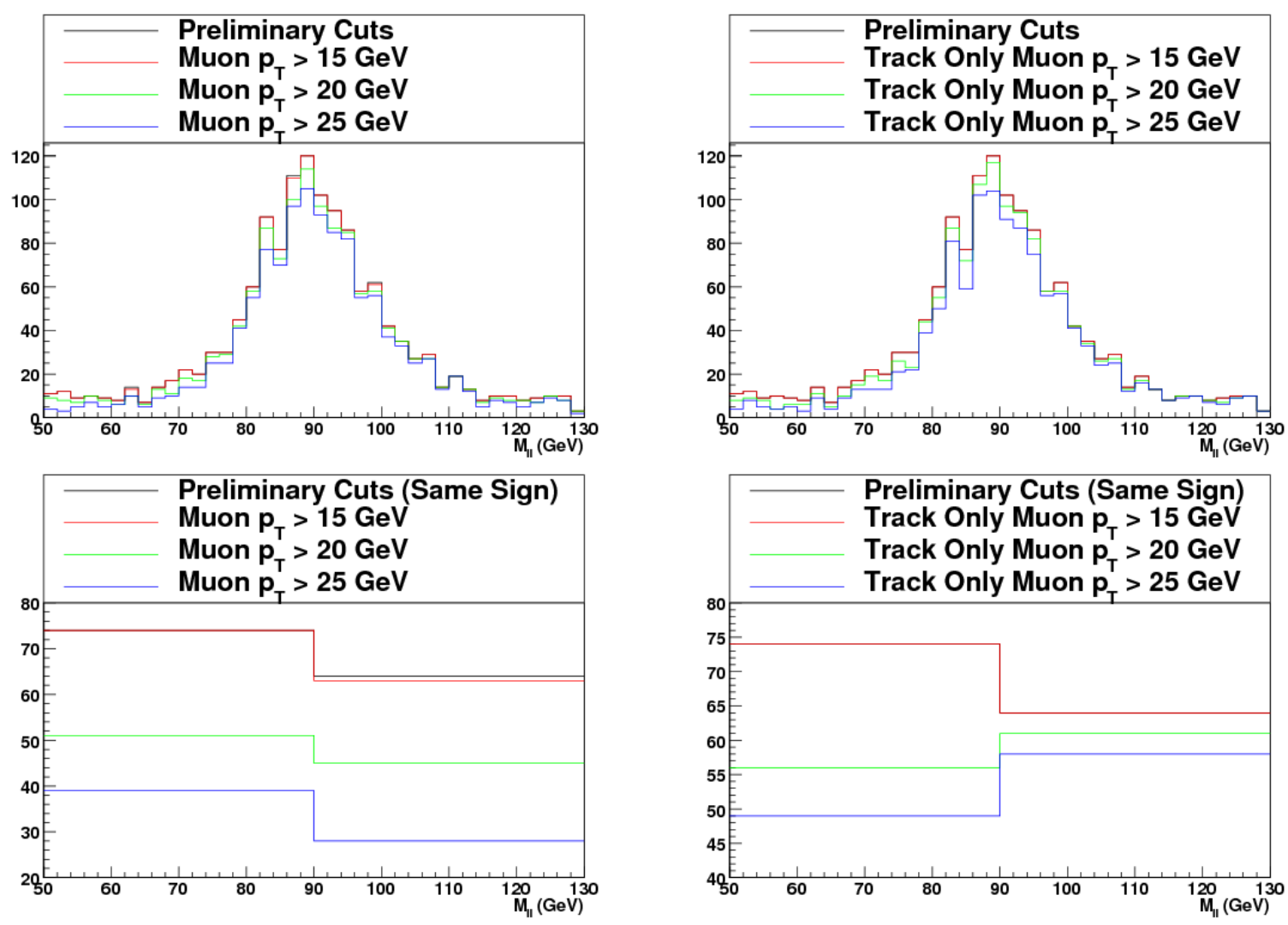

Figure 8.6: Di-lepton invariant mass, varying the cuts on $p_{T}$ of the muon and track only muon in signal like data (top) and QCD like data (bottom). The "Preliminary Cuts" distribution refers to the data sample with all the other cuts applied.

muons.

\subsubsection{Jet Selection}

The event is required to contain two jets which satisfy the following requirements:

- Reconstructed by the JCCB cone algorithm with $\Delta R<0.5$

- JES corrected

- $p_{T}>15 \mathrm{GeV}$

- Lead Jet $p_{T}>20 \mathrm{GeV}$

- Detector $\eta<2.5$ 

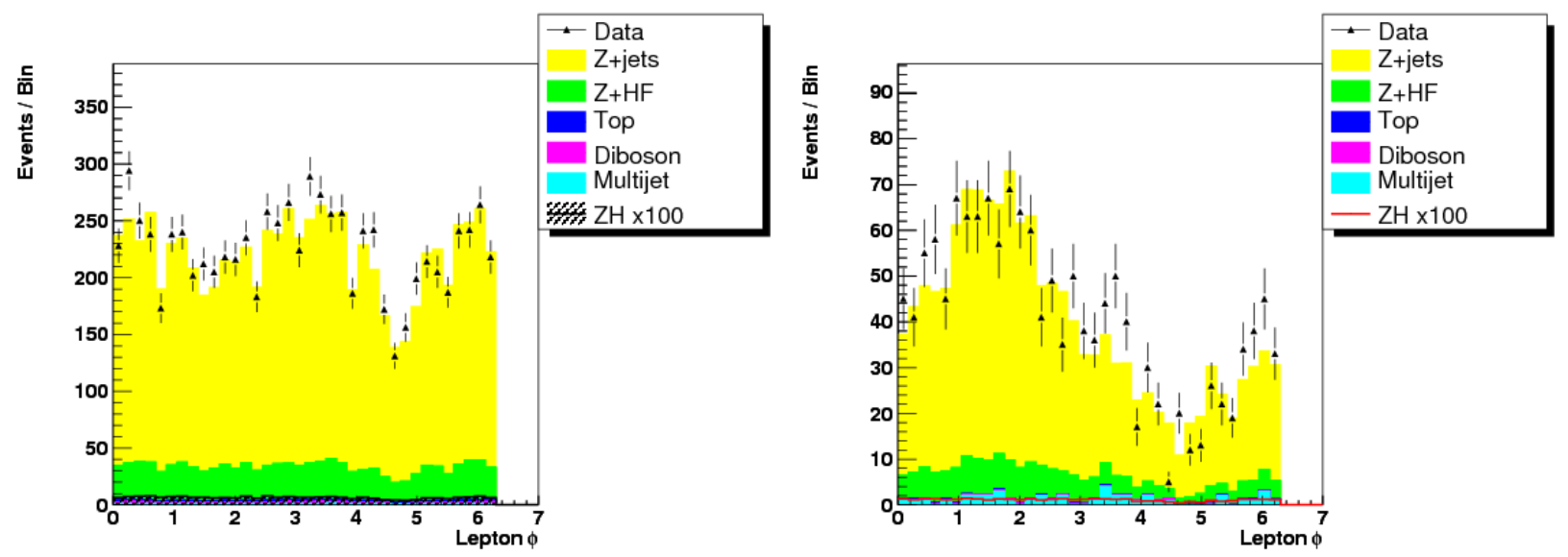

Figure 8.7: The $\phi$ distribution of the muons in the $Z H \rightarrow \mu \mu b b$ (left) and $Z H \rightarrow \mu+\operatorname{trk} b b$ (right) analyses.
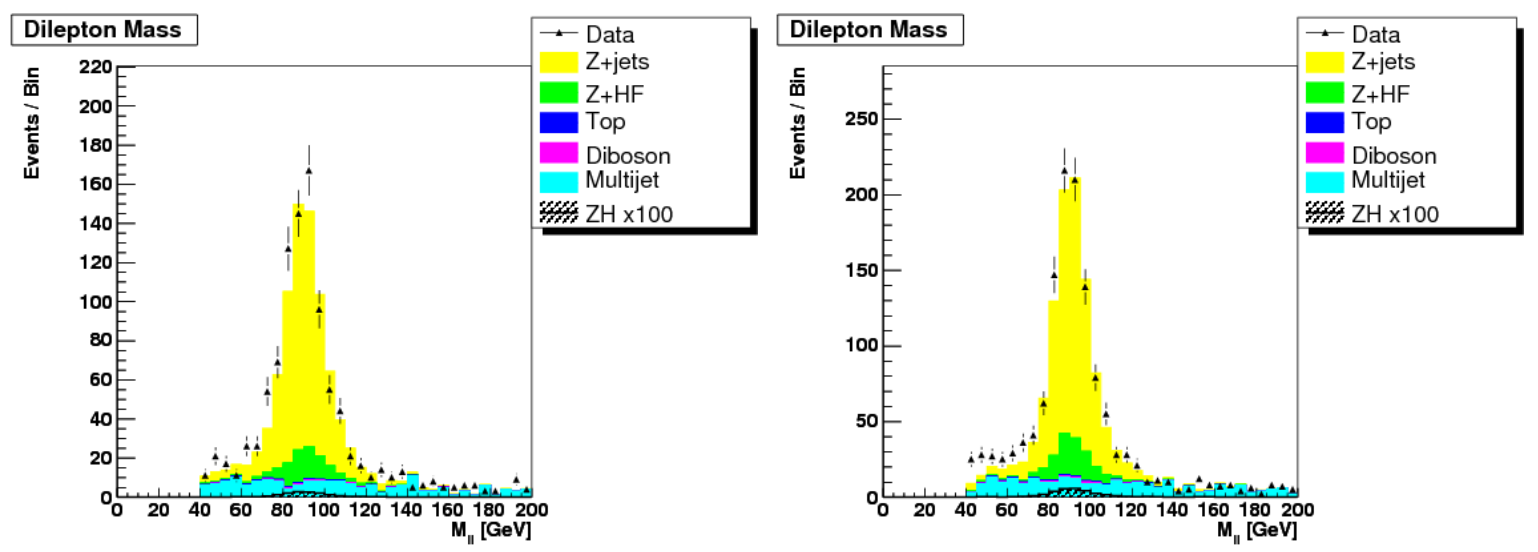

Figure 8.8: The di-lepton invariant mass in the Run IIa (left) and Run IIb (right) datasets after the pre-selection requirements have been applied.

Additionally, the sample is reduced by requiring either that one of the two jets satisfy a Tight NN B-Tag, or that both satisfy a VERYLOose NN B-Tag. B-Tagging is further described in Section 8.3. All three of the $Z H \rightarrow \ell \ell b b$ analyses use the same jet selection and b-tagging requirements.

The di-lepton invariant mass peak is shown in Figure 8.8 after the di-lepton and jets preselection requirements. 


\subsection{B-Tagging}

So far, we have made no experimental requirements on the parentage of the jets in the event. The preselection requirements can be refined to reject those backgrounds which produce light-flavored jets from $u, d$, and $s$ quarks using the methods described in Chapter 5. In so doing we substantially reduce a large fraction of the $Z+$ Jets and Multijet backgrounds.

We consider two types of $b$-tagged events.

1. Those with two or more jets which satisfy $b$-tagging requirements corresponding to the VERYLOOSE operating point (2L).

2. Those which do not satisfy the $2 \mathrm{~L}$ requirements are considered if one of the jets satisfies $b$-tagging requirements corresponding to the TIGHT operating point (1T).

The two samples are kept separate for all the rest of the steps in the analysis. Since the signal purity in the single $b$-tag sample is substantially reduced most of the sensitivity comes from the (2L) $b$-tag sample.

The selection requirements in data are relatively straightforward. If there are more than 2 VERYLOOSE $b$-tagged jets, we use the highest $p_{T}$ jets for the di-jet Higgs candidate and consider this a $2 \mathrm{~L}$ event. If there is only 1 Tight $b$-tagged jet, we use it and the highest $p_{T}$ non- $b$-tagged jet and consider this a $1 \mathrm{~T}$ event.

The MC requires a more sophisticated approach. We must first evaluate the probability that each jet in the event will pass either the loose or tight $b$-tagging requirements. From this, we can calculate the probability for the event to end up in the $1 \mathrm{~T}$ or $2 \mathrm{~L}$ sample. In this way, the same MC events are used in both samples, but with different event weights. The calculation of the event weights are described below.

\subsubsection{L Inclusive Weighting}

We define $L_{i}$ to be the probability that the $i^{\text {th }}$ jet satisfies the VERYLOOSE $b$-tag requirements. The probability that any two jets in an $n$ jet system both pass the $b$-tag requirements is given by Equation 8.1. Remember that we only consider the highest $p_{T} b$-tagged jets, which simplifies the combinatorial effects in events where more than two jets may have been tagged.

$$
p(i, j)=\frac{L_{i} L_{j}}{\left(1-L_{i}\right)\left(1-L_{j}\right)} \prod_{k=0}^{j}\left(1-L_{k}\right)
$$

\subsubsection{T Exclusive Weighting}

A probability, $T_{i}$, can also be computed for jets in the event to pass the Tight tagging requirements. We still need $L_{i}$ to evaluate the probability that none of the other jets have satisfied the VerYLOOSE tagging requirements. The (1T) event weight is given by Equation 8.2. 
Since we only use the highest $p_{T}$ un-tagged jet, the probability for any combination of jets not involving the lead jet is always zero. The $\delta_{j 1}$ is a delta function which returns one for $j=1$ and zero otherwise.

$$
p(0, j)=\left(\prod_{k=0}^{n}\left(1-L_{k}\right)\right)\left(\frac{T_{j}}{1-L_{j}}+\delta_{j 1} \times \frac{T_{0}}{1-L_{0}}\right)
$$



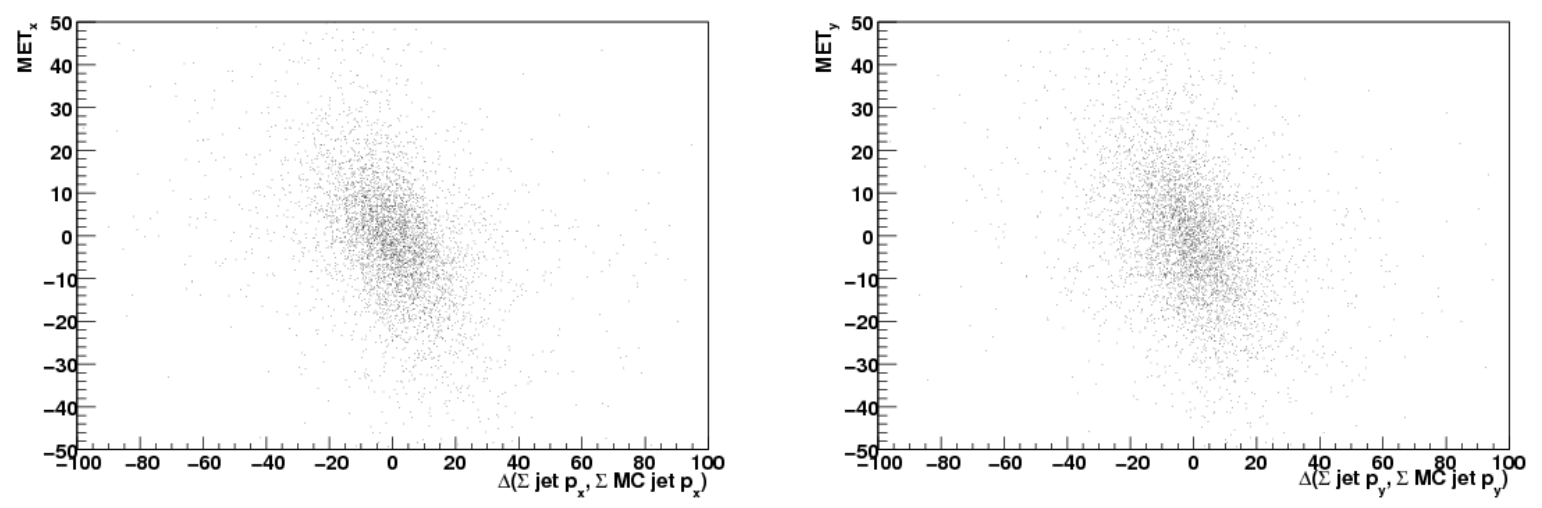

Figure 8.9: $\mathbb{E}_{T}$ plotted against the difference between the reconstructed and MC truth momentum of the jets in $Z H$ events with $M_{H}=115 \mathrm{GeV}$. The x-axis projection is on the left, the $\mathrm{y}$-axis projection on the right.

\subsection{Kinematic Fit}

The work presented in this section is based on the fact that the lepton energies are better modeled than those of the jets, and the $Z H$ system's boost is moderate for the vast majority of events. As seen in Figure 8.9, it is not unreasonable to assume that the missing transverse energy in the events is largely due to jet energy mismeasurements.

Given these points, we introduce a kinematic fit by $\chi^{2}$ minimization, fluctuating the energies and angles of the leptons and jets. The multi-dimensional minimization is performed by moving along the gradient of the $\chi^{2}$ defined as:

$$
\chi^{2}=\sum_{i}\left(\frac{x_{i}-x_{i}^{\prime}}{\sigma\left(x_{i}\right)}\right)^{2}+\sum_{j} \lambda_{j} c_{j}
$$

Here the $x_{i}$ are the $\eta, \phi$, and $p_{T}$ of the two $Z$-candidate leptons and the two $H$-candidate jets and $x_{i}^{\prime}$ are the fitted values. The $\sigma$ terms are the value uncertainties, parametrized in Appendix B. The $\lambda_{j}$ are the Lagrange multipliers for the three $c_{j}$ constraints. The constraints are as follows:

1. The di-lepton invariant mass is compatible with the $Z$ mass given its experimental width: $\left|M_{Z}-M_{\ell \ell}\right| / \Gamma_{Z}$

2. The sum of the momentum along the $x$ direction is compatible with zero, allowing for a $7 \mathrm{GeV}$ Gaussian width: $\left|\sum p_{x}\right| / 7.0 \mathrm{GeV}$

3. The sum of the momentum along the $y$ direction is compatible with zero, allowing for a $7 \mathrm{GeV}$ Gaussian width: $\left|\sum p_{y}\right| / 7.0 \mathrm{GeV}$ 
The width of the $p_{T}$ constraint was determined from MC truth information.

\subsubsection{Implementation}

The fit is performed by the Conjugate Gradient Method [51], designed to solve $N \times N$ linear systems of equations. The algorithm generates a succession of "search directions" in $\chi^{2}$-space based on the idea of minimizing the function:

$$
f(\boldsymbol{x})=\frac{1}{2} \boldsymbol{x} \cdot \boldsymbol{A} \cdot \boldsymbol{x}-\boldsymbol{b} \cdot \boldsymbol{x}
$$

which happens when the gradient is:

$$
\nabla f=\boldsymbol{A} \cdot \boldsymbol{x}-\boldsymbol{b}=0
$$

The algorithm can be generalized to arbitrary non-linear functions and can be summarized as follows:

1. Initialize the "well-measured" parameter vector $\boldsymbol{x}_{k}$ with the $\eta$ and $\phi$ of the four objects. Initialize the "poorly-measured" parameter vector $\boldsymbol{y}_{k}$ with the $p_{T}$ of the four objects.

2. Fill the $5 \times 3$ matrices $B x_{k}$ and $B y_{k}$ with the gradient of the $\chi^{2}$ with respect to the $\boldsymbol{x}_{k}$ and $\boldsymbol{y}_{k}$ components.

3. Solve the linearized system for the new values of the Lagrange multipliers $\left(\alpha_{k}\right)$.

4. Calculate a step along the $\chi^{2}$ plane $\left(\boldsymbol{p}_{k}\right)$ which minimizes the function $\boldsymbol{x}_{k}+\alpha_{k} \boldsymbol{p}_{k}$.

5. Once again, solve for the Lagrange multipliers $\left(\beta_{k}\right)$

6. Find a new step along the $\chi^{2}$ plane $\boldsymbol{s}_{k}=-\boldsymbol{p}_{k}+\beta_{k-1} \boldsymbol{s}_{k-1}$. $\boldsymbol{s}_{0}$ is set to $\boldsymbol{p}_{k}$.

7. If the change in $\chi^{2}$ since the last step is below a threshold value, and the constraints are better satisfied, exit. Otherwise, return to step 2.

If the fit has not converged after 300 iterations, it fails.

\subsubsection{Fit Performance}

The ratio of $\frac{\text { MCtruth }}{p_{T}}$ is shown in Figure 8.10 for the leading and second-leading jets before and after the kinematic fit. The resolution of the jets $\left(\frac{\mathrm{RMS}}{\text { Mean }}\right)$ in the signal is moderately improved, by 10 to $20 \%$ for each of the Higgs mass bins. Since the shifts in two jets' $p_{T}$ are correlated and the effect is multiplicative, this translates into an average improvement of $40 \%$ in the 

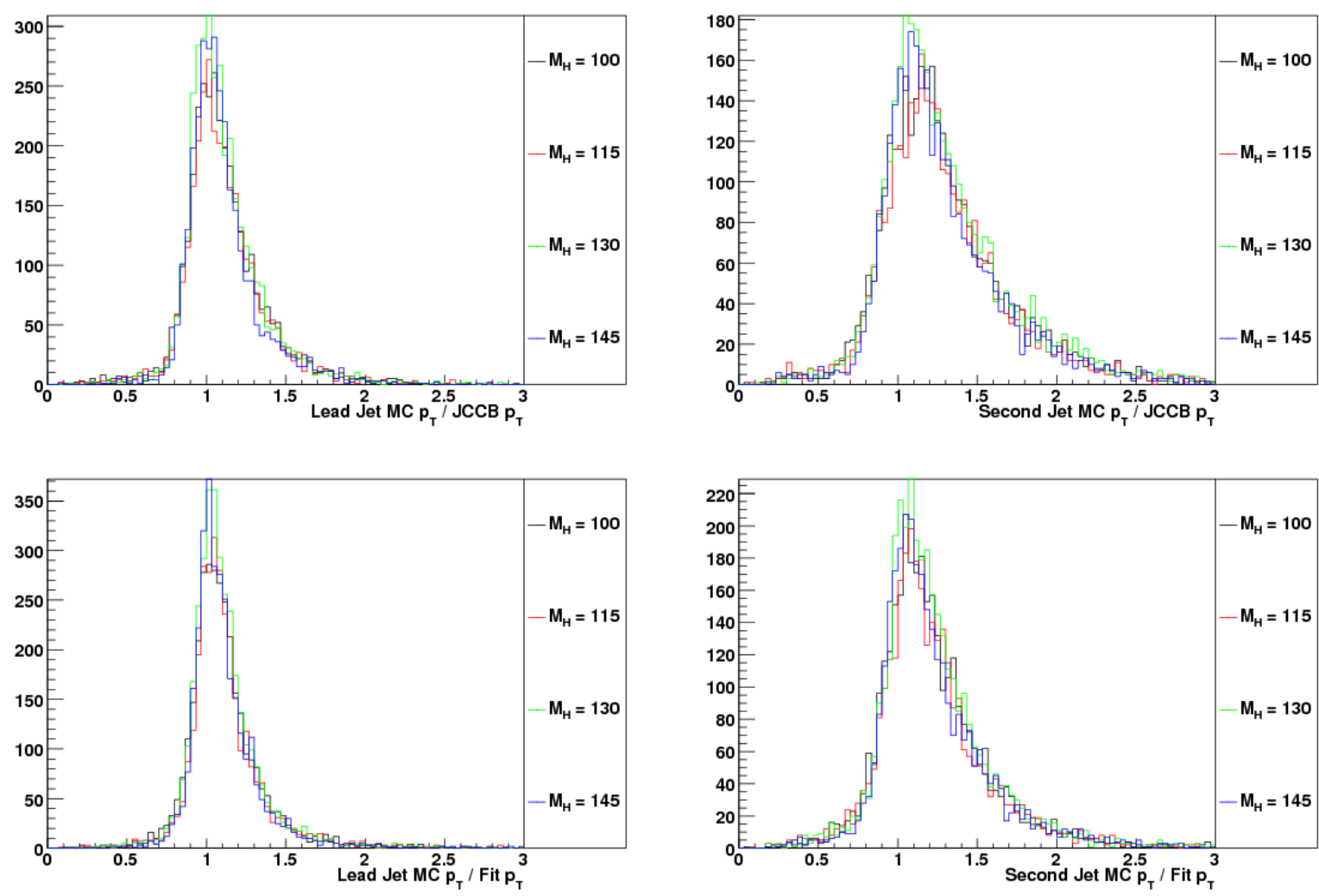

Figure 8.10: Jet MC $p_{T}$ truth / $p_{T}$ in $\mathrm{ZH} \mathrm{MC} \mathrm{for} \mathrm{100,} \mathrm{115,} \mathrm{130,} \mathrm{and} 145 \mathrm{GeV}$ Higgs masses for the leading (left) and second (right) jets, before (top) and after (bottom) applying the kinematic fit.

di-jet invariant mass resolution. The RMS and Mean of these three distributions are listed in Tables 8.4, 8.5, and 8.6 for each of the Higgs mass bins.

Although the signal resolution is only slightly improved, Figure 8.11 shows that the invariant mass distribution of the background samples are broadened. These two effects work together and the signal to background ratio is significantly improved after the fit. Without considering the effect of systematics, this translates into an $8 \%$ improvement in the expected limit calculated using the di-jet invariant mass distribution.

These improved kinematic variables will be used as inputs to the multi-variate discriminant (MVA) described in Section 8.6.

\subsubsection{Cutting on the fit $\chi^{2}$}

Additionally, as shown in Figure 8.12, the $\chi^{2}$ provides an additional level of rejection for the multijet and $t \bar{t}$ backgrounds. We observe that to satisfy the constraints of the kinematic fit 
Table 8.4: Mean and RMS of the leading jet's $\frac{p_{T_{M C}}}{p_{T_{\text {reco }}}}$ distribution in $Z H$ MC.

\begin{tabular}{|c|ccc|ccc|}
\hline \hline & \multicolumn{3}{|c|}{ Pre Fit } & \multicolumn{3}{c|}{ Post Fit } \\
$M_{H}(\mathrm{GeV})$ & mean & rms & rms/mean & mean & rms & rms/mean \\
\hline 100 & 0.95 & 0.24 & 0.25 & 0.93 & 0.20 & 0.21 \\
105 & 0.94 & 0.23 & 0.24 & 0.93 & 0.19 & 0.21 \\
110 & 0.94 & 0.22 & 0.23 & 0.94 & 0.18 & 0.20 \\
115 & 0.93 & 0.22 & 0.24 & 0.93 & 0.19 & 0.20 \\
120 & 0.94 & 0.22 & 0.23 & 0.93 & 0.19 & 0.20 \\
125 & 0.94 & 0.21 & 0.22 & 0.94 & 0.18 & 0.19 \\
130 & 0.94 & 0.20 & 0.21 & 0.93 & 0.19 & 0.21 \\
135 & 0.94 & 0.20 & 0.21 & 0.93 & 0.17 & 0.18 \\
140 & 0.94 & 0.21 & 0.22 & 0.93 & 0.17 & 0.18 \\
145 & 0.94 & 0.19 & 0.20 & 0.93 & 0.17 & 0.18 \\
150 & 0.94 & 0.19 & 0.20 & 0.93 & 0.17 & 0.18 \\
\hline \hline
\end{tabular}

these two backgrounds tend to have to significantly skew the original momenta of the leptons and jets. The muon and jet energy pull plots, which are essentially the sigma deviation plots for the variable in question, are shown in Figure 8.13 and Figure 8.14. Whereas the signal distribution is centered around zero with a width of $\approx 1 \sigma$, the multijet and $t \bar{t}$ distributions are closer to the edges of the plot.

The agreement between data and $\mathrm{MC}$ in the $\chi^{2}$ plot leaves something to be desired, so the variable will not be fed to the MVA. Instead, we apply a direct cut, determined individually for each of the Higgs masses. This cut removes $92 \%$ of the multijet background but only $17 \%$ of the signal in the $m_{H}=115 \mathrm{GeV}$ bin. This is especially useful since the track-only muon channel is much more sensitive to the multijet background than any of the three other $Z H \rightarrow \ell \ell b b$ analyses. 
Table 8.5: Mean and RMS of the second leading jet's $\frac{p_{T_{M C}}}{p_{T_{\text {reco }}}}$ distribution in $Z H$ MC.

\begin{tabular}{|c|ccc|ccc|}
\hline \hline & \multicolumn{3}{|c|}{ Pre Fit } & \multicolumn{3}{c|}{ Post Fit } \\
$M_{H}(\mathrm{GeV})$ & mean & rms & rms/mean & mean & rms & rms/mean \\
\hline 100 & 0.84 & 0.27 & 0.32 & 0.88 & 0.26 & 0.29 \\
105 & 0.83 & 0.28 & 0.33 & 0.87 & 0.27 & 0.31 \\
110 & 0.83 & 0.26 & 0.31 & 0.87 & 0.25 & 0.28 \\
115 & 0.84 & 0.27 & 0.33 & 0.88 & 0.27 & 0.30 \\
120 & 0.84 & 0.27 & 0.33 & 0.87 & 0.27 & 0.31 \\
125 & 0.84 & 0.27 & 0.32 & 0.87 & 0.25 & 0.28 \\
130 & 0.83 & 0.27 & 0.32 & 0.87 & 0.25 & 0.29 \\
135 & 0.84 & 0.27 & 0.32 & 0.88 & 0.25 & 0.28 \\
140 & 0.84 & 0.25 & 0.30 & 0.87 & 0.23 & 0.27 \\
145 & 0.84 & 0.26 & 0.31 & 0.87 & 0.24 & 0.28 \\
150 & 0.83 & 0.25 & 0.30 & 0.86 & 0.23 & 0.26 \\
\hline \hline
\end{tabular}

Table 8.6: Mean and RMS of the di-jet $\frac{M_{j j_{M C}}}{M_{j j_{\text {reco }}}}$ distribution in $Z H$ MC.

\begin{tabular}{|c|ccc|ccc|}
\hline \hline & \multicolumn{3}{|c|}{ Pre Fit } & \multicolumn{3}{c|}{ Post Fit } \\
$M_{H}(\mathrm{GeV})$ & mean & rms & rms/mean & mean & rms & rms/mean \\
\hline 100 & 1.02 & 0.10 & 0.10 & 1.00 & 0.06 & 0.06 \\
105 & 1.01 & 0.10 & 0.10 & 1.00 & 0.06 & 0.06 \\
110 & 1.01 & 0.10 & 0.10 & 1.00 & 0.06 & 0.06 \\
115 & 1.02 & 0.11 & 0.10 & 1.00 & 0.06 & 0.06 \\
120 & 1.02 & 0.11 & 0.10 & 1.00 & 0.06 & 0.06 \\
125 & 1.01 & 0.11 & 0.11 & 1.00 & 0.07 & 0.07 \\
130 & 1.01 & 0.11 & 0.11 & 1.00 & 0.07 & 0.07 \\
135 & 1.01 & 0.11 & 0.11 & 1.00 & 0.06 & 0.06 \\
140 & 1.02 & 0.11 & 0.11 & 1.00 & 0.06 & 0.06 \\
145 & 1.01 & 0.11 & 0.11 & 1.00 & 0.06 & 0.06 \\
150 & 1.02 & 0.11 & 0.11 & 1.00 & 0.06 & 0.06 \\
\hline \hline
\end{tabular}




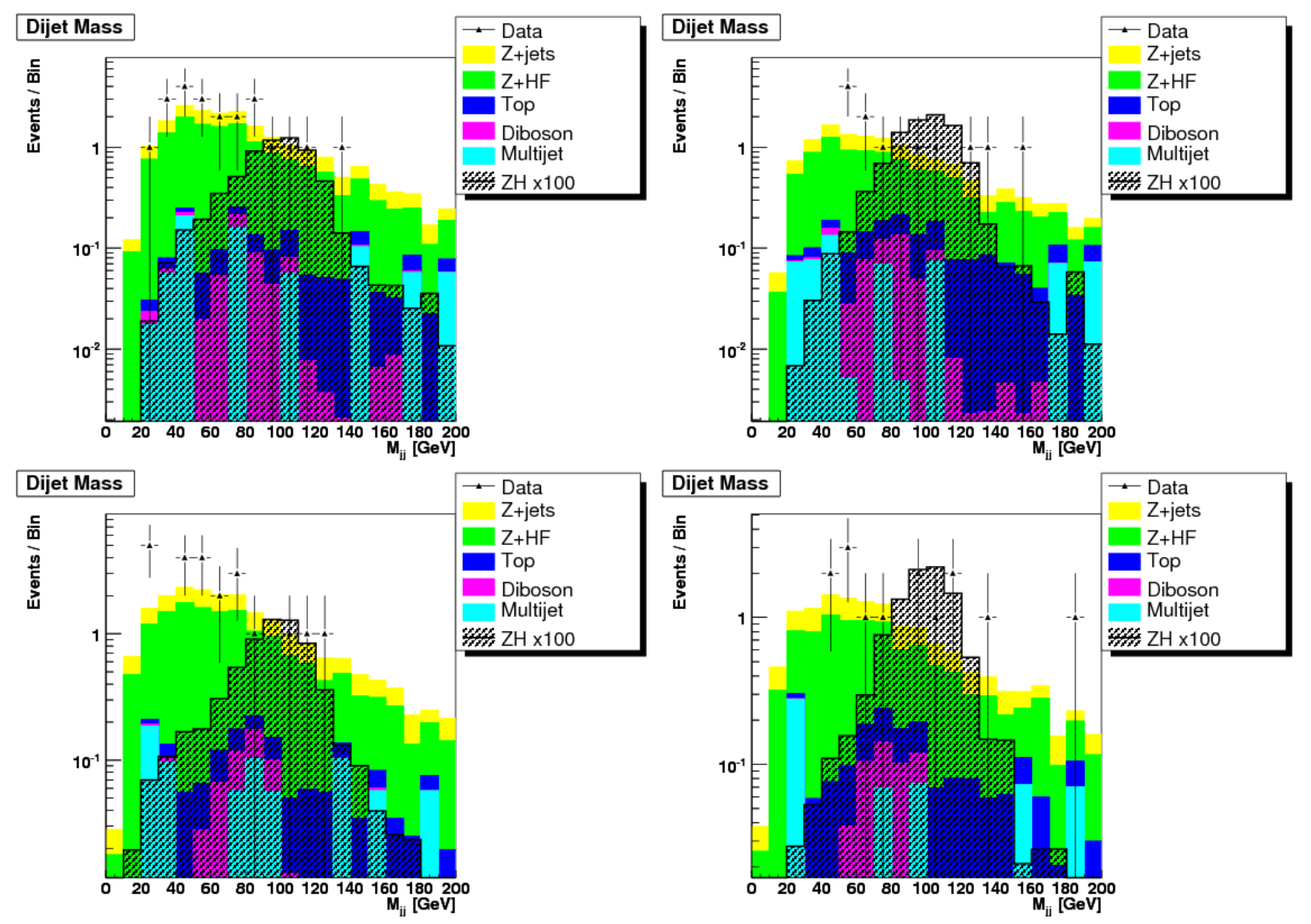

Figure 8.11: Di-jet invariant mass in the 1T (left) and 2L $b$-tag samples before (top) and after (bottom) applying the kinematic fit in the Run IIb dataset. 

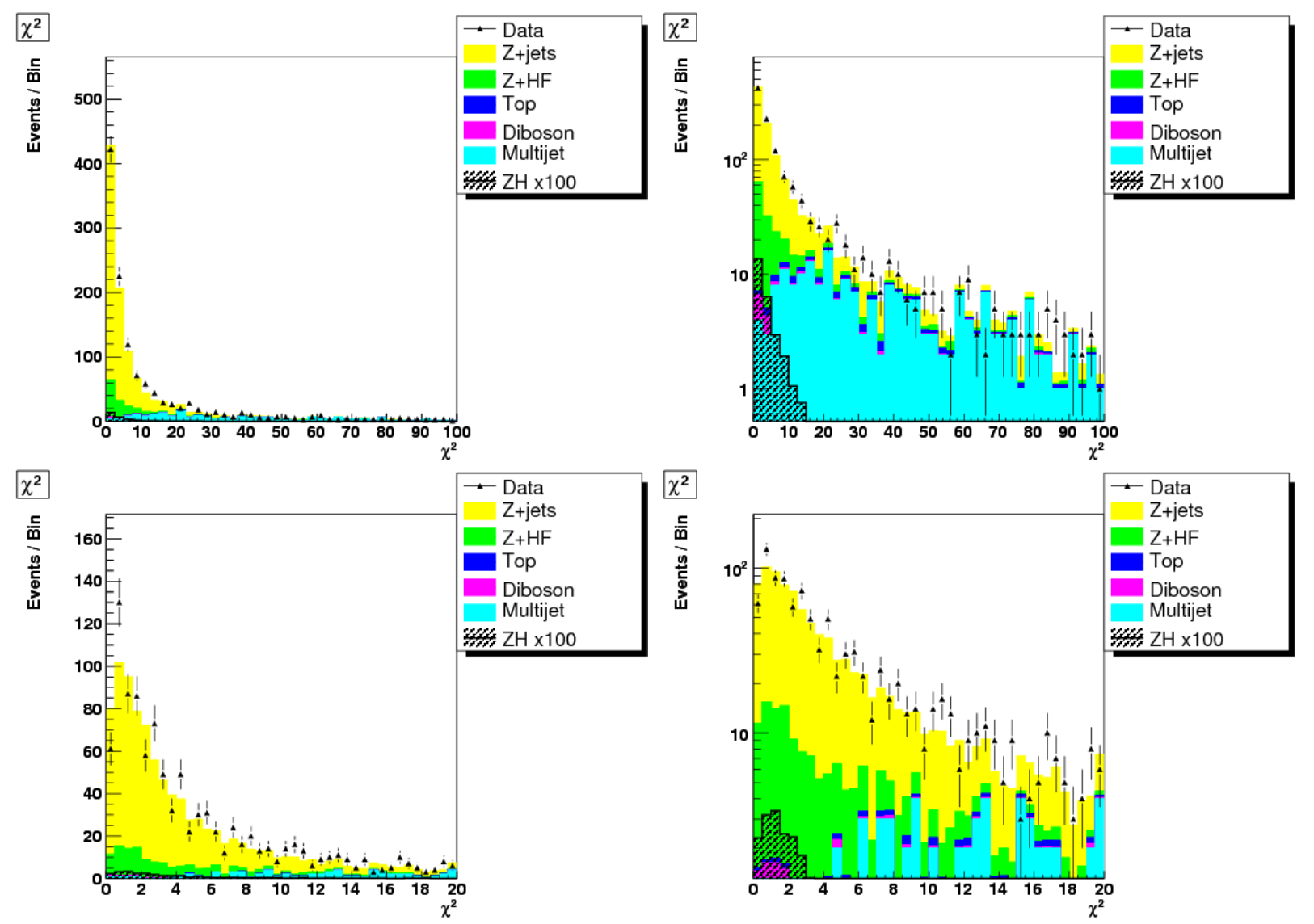

Figure 8.12: The kinematic fit's $\chi^{2}$ distribution over different ranges in linear and log scales. 

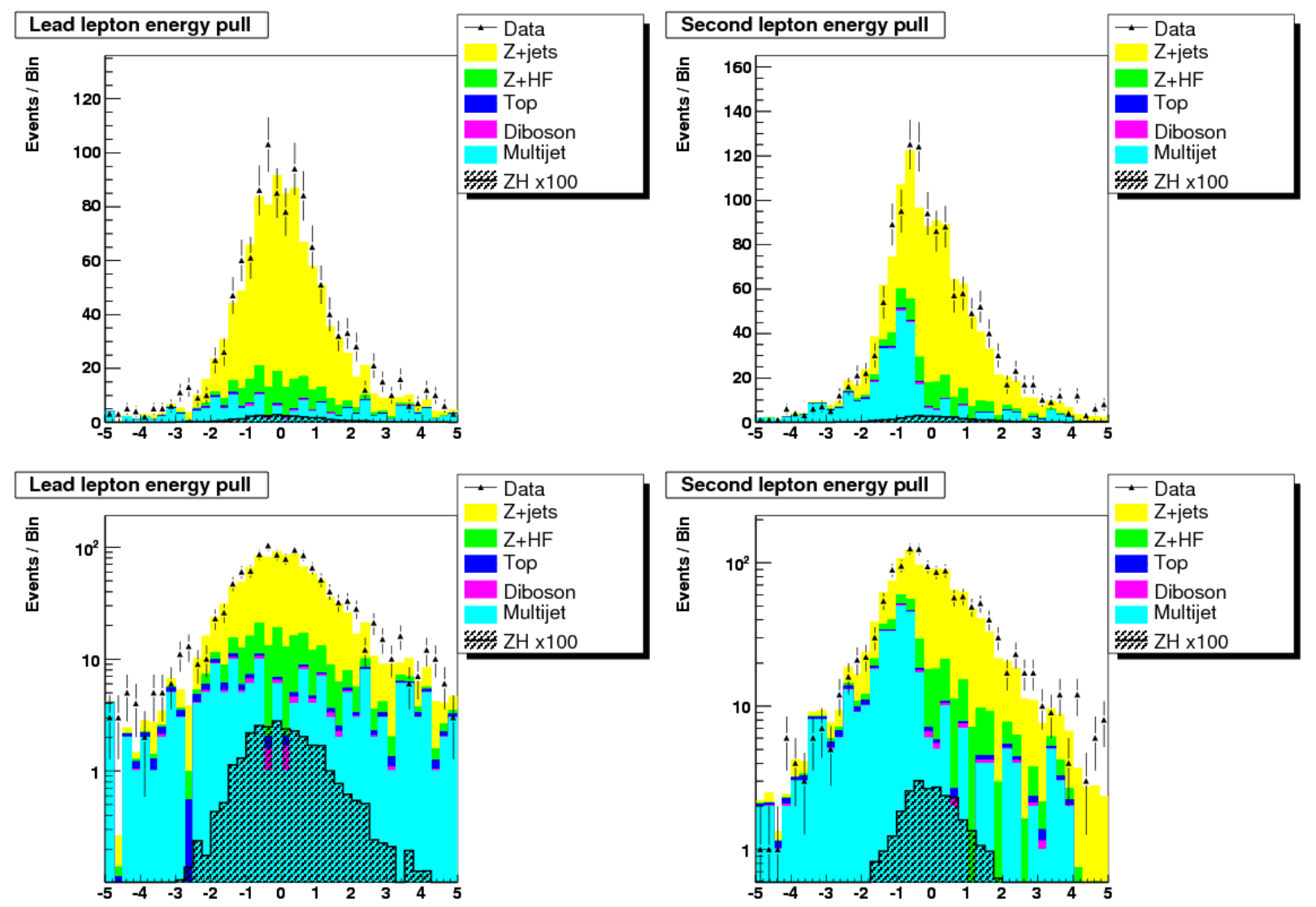

Figure 8.13: The kinematic fit pull plot for the energy of the muon (left) and track-only muon (right). 

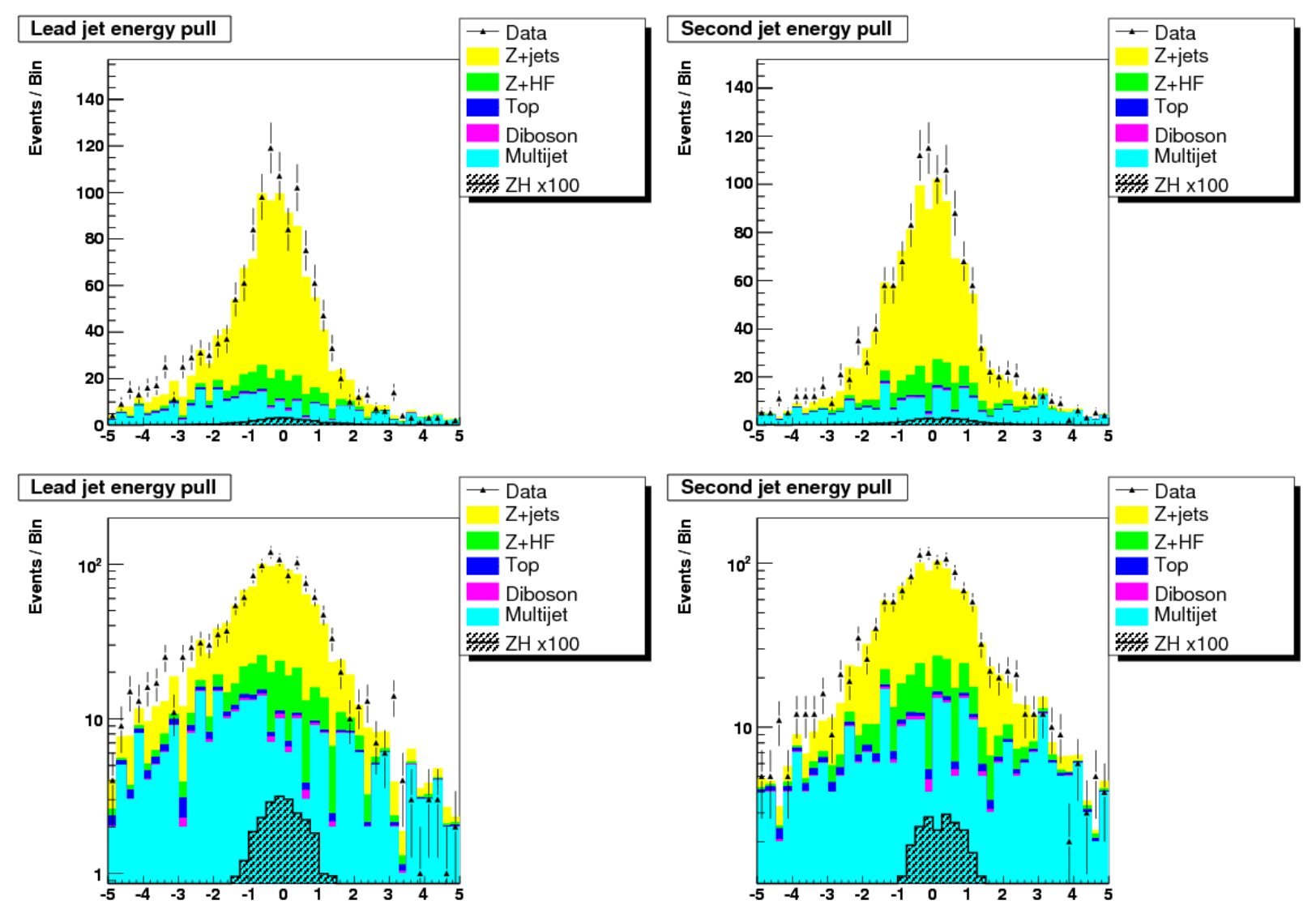

Figure 8.14: The kinematic fit pull plot for the energy of the lead (left) and second (right) jet. 


\subsection{Yields}

We now evaluate the predicted yields from MC and compare them to the data.

\begin{tabular}{|l|cccc|}
\hline \hline \multicolumn{5}{c|}{ Run IIa } \\
\hline & pre-selection & $70<M_{\mu \mu}<130 \mathrm{GeV}$ & 1 tight b-tag & 2 loose b-tags \\
\hline \hline data & 1149 & 591 & 20 & 7 \\
all bkg & $1049 \pm 18$ & $585 \pm 6$ & $12.8 \pm 0.3$ & $6.6 \pm 0.1$ \\
Multijet & $285 \pm 17$ & $16 \pm 4$ & $0.88 \pm 0.2$ & $0.21 \pm 0.06$ \\
$Z j j$ & $651 \pm 5$ & $492 \pm 4$ & $2.41 \pm 0.02$ & $1.31 \pm 0.01$ \\
$Z b \bar{b}$ & $27.6 \pm 0.6$ & $21.0 \pm 0.5$ & $5.67 \pm 0.2$ & $2.83 \pm 0.1$ \\
$Z c \bar{c}$ & $62.4 \pm 1$ & $48.2 \pm 1$ & $2.97 \pm 0.08$ & $1.25 \pm 0.05$ \\
$Z Z$ & $4.12 \pm 0.1$ & $3.28 \pm 0.1$ & $0.276 \pm 0.02$ & $0.242 \pm 0.02$ \\
$W Z$ & $4.16 \pm 0.2$ & $3.13 \pm 0.2$ & $0.114 \pm 0.01$ & $0.0366 \pm 0.005$ \\
$W W$ & $2.66 \pm 0.1$ & $0.264 \pm 0.04$ & $0.00282 \pm 0.0006$ & $0.00142 \pm 0.0003$ \\
$t \bar{t}$ & $13.06 \pm 0.09$ & $1.75 \pm 0.03$ & $0.498 \pm 0.01$ & $0.731 \pm 0.02$ \\
$Z H(115)$ & $0.152 \pm 0.003$ & $0.119 \pm 0.002$ & $0.0350 \pm 0.0007$ & $0.0447 \pm 0.001$ \\
\hline \hline
\end{tabular}

\begin{tabular}{|l|cccc|}
\hline \hline \multicolumn{5}{c|}{ Run IIb } \\
\hline & pre-selection & $70<M_{\mu \mu}<130 \mathrm{GeV}$ & 1 tight b-tag & 2 loose b-tags \\
\hline \hline data & 1435 & 796 & 22 & 14 \\
all bkg & $1342 \pm 22$ & $782 \pm 11$ & $22.2 \pm 0.4$ & $14.5 \pm 0.3$ \\
Multijet & $374 \pm 19$ & $14 \pm 4$ & $0.880 \pm 0.3$ & $0.710 \pm 0.2$ \\
$Z j j$ & $805 \pm 11$ & $652 \pm 10$ & $6.04 \pm 0.1$ & $3.89 \pm 0.06$ \\
$Z b \bar{b}$ & $39.1 \pm 1$ & $30.4 \pm 0.9$ & $8.5 \pm 0.3$ & $5.44 \pm 0.2$ \\
$Z c \bar{c}$ & $89.8 \pm 2$ & $72.7 \pm 2$ & $5.51 \pm 0.2$ & $2.77 \pm 0.1$ \\
$Z Z$ & $5.56 \pm 0.2$ & $4.36 \pm 0.2$ & $0.339 \pm 0.02$ & $0.405 \pm 0.04$ \\
$W Z$ & $6.34 \pm 0.330$ & $4.77 \pm 0.3$ & $0.212 \pm 0.02$ & $0.0803 \pm 0.006$ \\
$W W$ & $2.58 \pm 0.4$ & $0.536 \pm 0.2$ & $0.00680 \pm 0.002$ & $0.00431 \pm 0.001$ \\
$t \bar{t}$ & $19.3 \pm 0.2$ & $2.87 \pm 0.1$ & $0.770 \pm 0.03$ & $1.21 \pm 0.04$ \\
$Z H(115)$ & $0.298 \pm 0.007$ & $0.239 \pm 0.006$ & $0.0646 \pm 0.002$ & $0.0948 \pm 0.003$ \\
\hline \hline
\end{tabular}

Table 8.7: The data, backgrounds, and expected SM signal after the two jet and muon+track mass $\left(60<M_{\ell \ell}<150 \mathrm{GeV}\right)$ requirements. A cut of the fit $\chi^{2}<9$ is applied at the same time as the mass cut. After applying $b$-tagging, the samples are divided into $2 \mathrm{~L}$ and $1 \mathrm{~T}$ samples. 


\subsection{Multivariate Discriminant}

Previous versions of the $Z H \rightarrow \ell \ell b b$ search have used, amongst others, Artificial Neural Networks (ANN) and Boosted Decision Tree (BDT) classifiers. This latest version uses a Random Forest, which provides substantial improvements in performance and training stability. The last decade has seen the rise of new multivariate techniques, motivated by advances in machine learning algorithms which help to squeeze even more sensitivity out of our inputs. While a traditional discriminant, like the likelihood method which was used in Section 7.6, machine learning algorithms provide an additional gain in sensitivity by exploiting minute differences and correlations in multiple inputs.

\subsubsection{Random Forest}

A Random Forest (RF) [52] is an extension of the Decision Tree classifier type. A simple Decision Tree would be a succession of rectangular cuts on a list of variables $\boldsymbol{x}$. At each level $(i)$, the events are examined to see if they pass some threshold value $(\boldsymbol{c})$. The threshold values on the variable under consideration are unique for each bifurcation such that at each level the test with the highest selection purity is used on the node and the following test is performed: $\eta_{i}\left(x_{n}\right)=x_{n}<c_{i}$. Each of the daughter nodes are assigned a label, "signal" or "background." Initially, each node will have some amount of mis-classified events: either signal in a background node, or background in a signal node. This process of cuts is repeated until some stopping criterion, typically based on the mix of signal to background or the number of events in the terminal nodes. In this implementation, we use the Gini Index $(\mathrm{Q}(\mathrm{p}))$ as the figure of merit. It is defined as:

$$
Q(p)=-2 p(1-p)
$$

where $p$ is the fraction of events correctly classified as signal or background and each cut is selected so as to maximize this value.

The disadvantage of this method is that each successive level becomes more statistically limited, and more importantly, the information about events which descend a different path is lost. If a cut is set too stringently, the algorithm will fail to find an optimal set of final nodes, which can lead to a level of training instability.

We wish to retain the functionality of the Decision Tree but harden it against training instabilities. In a $\mathrm{RF}$ we train a large number $(K)$ of Decision Trees, varying their input variables and events. The idea is that each individual Decision Tree remains susceptible to over-training, but the instabilities are smoothed out by varying the training parameters and averaging over the full set. Two features are used to permutate the training of the Decision Trees. 


\section{Random Subspace Sampling}

The list of input variables for each of the $K$ Decision Trees are chosen by Random Subspace Sampling. Essentially, a sub-set of $m$ variables is randomly selected from the $n$-sized list of available ones. As the Decision Tree iteratively creates its branches, each unique sub-set of available variables will generate a different optimum set of cuts. Nominally, this has the advantage of reducing the classifier's instability with respect to the correlations between variables.

\section{Bagging}

In Bagging (Bootstrap AGGregatING), each tree is grown from a random selection of training events corresponding to a fraction $(s)$ of the total training sample. The method includes the possibility that an event might be randomly picked multiple times. When interpreting the training sample as analogous to a parton distribution function, this double-counting is the equivalent of the non-negligible possibility of having picked the same random number from a high-probability region of the phase-space. Again, the individual trees are subject to training instabilities, but the risk of over-training is once again smoothed out over the full set of trees.

\subsubsection{Technical Implementation}

The RF was implemented by the TMVA software package [53]. Separate RFs are trained for each of the physics channels $(\mu \mu, \mu+t r k, e e, e+I C R)$, for each of the Higgs mass points $\left(100 \leq m_{H} \leq 150 \mathrm{GeV}\right.$, in $5 \mathrm{GeV}$ increments), and for each of the tag points (2L and 1T).

In each case, the MC dataset is split into three parts. Half is set aside for the final estimation of the search sensitivity, a quarter is used to train the RF, and another quarter is used to test the $\mathrm{RF}$ for over-training. We set the value of $s=0.5$ for the Bagging, use a random subset of $m=19$ input variables, and train over 200 trees.

\subsubsection{Variable Selection}

The split training procedure means that a RF can accept a much larger number of well modeled input variables than likelihoods or Neural Nets without deteriorating the performance of the classifier. The time required to train the RF does however grow quickly as the number of inputs increases. Since we wish to visually examine each input variable distribution, decreasing the number of inputs saves both our time and our sanity.

A reproducible method is implemented to evaluate the level of agreement between the Data and $\mathrm{MC}$ distributions. To be considered as input for the RF, a variable distribution must pass cuts on both of the two following criteria.

- Kolmogorov-Smirnov Probability $>0.05$ : A test of the shape compatibility between two distributions. It is however very sensitive to the choice of binning for the histograms. 


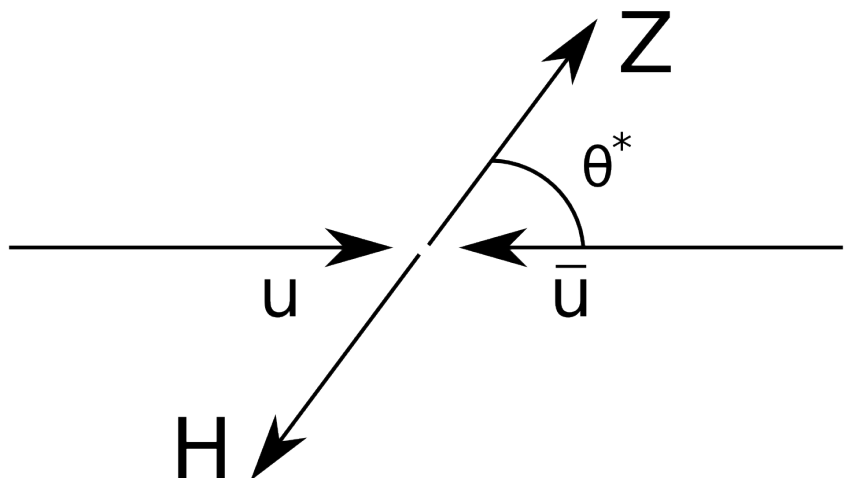

Figure 8.15: A diagram of $\theta^{*}$, the angle between the $u$-quark and the $Z$ boson.

- $\chi^{2}$ Probability $>0.05$ : The number of events per bin are expected to be randomly drawn from a Poisson distribution. The $\chi^{2}$ evaluates by how many standard deviations the two distributions disagree over the entire range.

The 42 variables which satisfy the previous two requirements must also bring some measure of discrimination against the background. To this end, we iteratively remove 5 variables from the $\mathrm{RF}$ and retrain. This continues until the performance is reduced by more than $2 \%$, which in our case means that 3 rounds of removal are performed, leaving 37 variables. Each of the final variables is listed in Table 8.8 along with the fraction of nodes in which they are used.

Two new variables are considered: $\cos \left(\chi^{*}\right)$ and $\cos \left(\theta^{*}\right)$. Numerical methods are used to solve for the rest frame of the $b \bar{b}$ and $Z$ system in the event [54]. $\theta^{*}$ is defined as the angle between the $u$-quark (from the $p \bar{p}$ ) and the $Z$-candidate in the zero momentum frame. The system is then rotated into the $p \bar{p}$ collision frame in which $\chi^{*}$ is the angle between the $b \bar{b}$ system and the negatively charged lepton. Figure 8.15 illustrates the $\cos \left(\theta^{*}\right)$ variable. Although $\cos \left(\chi^{*}\right)$ is removed by our iterative variable selection process in the $m u+t r k$ channel, it is found to be useful in the di-muon and di-electron channels.

\subsubsection{RF Performance}

To understand the performance increase due to the use of a RF, we calculate 95\% C.L. limits, as described in Section 8.8, using the di-jet invariant mass and the RF output, ignoring the systematics. The fractional change in the expected limits in the mu+track channel is shown graphically in Figure 8.16, and listed in Table 8.9. The advantage of these multi-variate techniques is clearly demonstrated. For a low mass Higgs boson, we can expect a gain of up to $24 \%$ in this channel's sensitivity. 


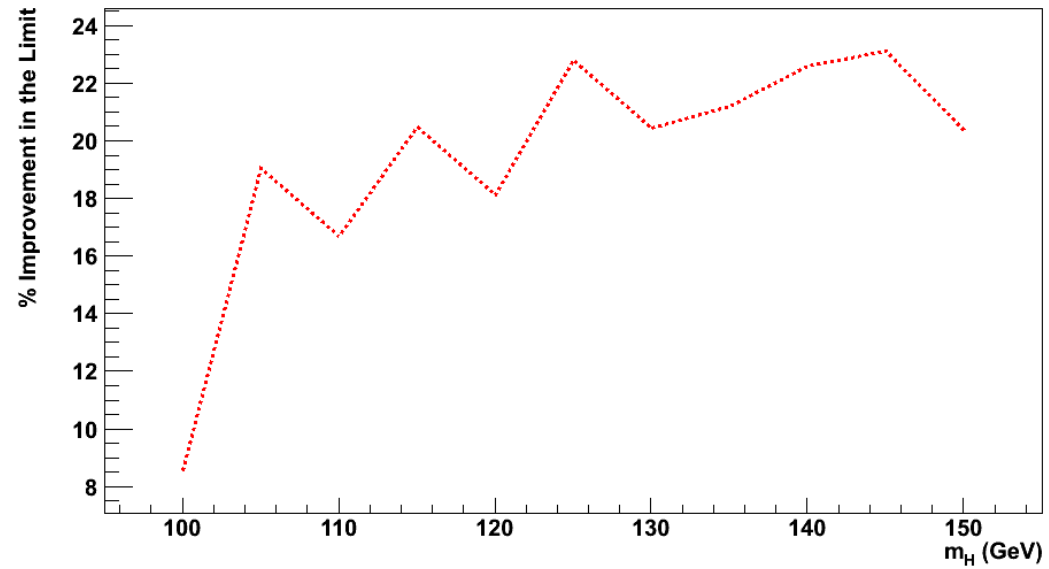

Figure 8.16: The percentage change in the expected $95 \%$ confidence limits for the mu+track channel (combining Run IIa and Run IIb) achieved by using the RF output instead of the di-jet invariant mass. The limits are calculated ignoring systematics. 
Table 8.8: The variables used in the final RF classifier and the fraction of times the variable is used in a node for the Run IIa and Run IIb $2 \mathrm{~L}$ and $1 \mathrm{~T}$ samples. The variables are sorted by the average of the four columns. $\Delta \phi$ is the angular seperation in $\phi, \Delta R$ is the seperation in $R$, $M_{x x}$ is the invariant mass of the two $x$ objects, $M_{T}^{x x}$ is the transverse component of $M_{x x}, b_{1}$ is the leading b-tagged jet, $b_{2}$ is the second leading (leading) b-tagged (untagged) jet in the $2 \mathrm{~L}$ (1T) sample, $\Delta \eta$ is the seperation in $\eta, \Delta \theta$ is the seperation in $\theta, Z H$ refers to the four-object system containing the two leptons and two jets, $\ell_{1}$ is the muon, $\ell_{2}$ is the track-only muon, $\ell \ell$ is the di-lepton system, $b b$ is the di-jet system, and (fit) refers to the quantity after the kinematic fit.

\begin{tabular}{|l|cccc|}
\hline \hline variable & Run IIb 2L & Run IIb 1T & Run IIa 2L & Run IIa 1T \\
\hline$\Delta \phi(\ell, \ell)$ & $2.18 \times 10^{-1}$ & $5.03 \times 10^{-1}$ & $3.55 \times 10^{-1}$ & $8.51 \times 10^{-1}$ \\
$M_{b b}($ fit $)$ & $3.55 \times 10^{-1}$ & $3.32 \times 10^{-1}$ & $1.80 \times 10^{-1}$ & $1.49 \times 10^{-2}$ \\
$\Delta R(\ell, \ell)$ & $2.50 \times 10^{-1}$ & $5.37 \times 10^{-2}$ & $2.61 \times 10^{-1}$ & $5.57 \times 10^{-2}$ \\
$M_{b b}$ & $1.43 \times 10^{-1}$ & $4.92 \times 10^{-2}$ & $8.95 \times 10^{-2}$ & $1.32 \times 10^{-2}$ \\
$\Delta \phi(\mathrm{Z}, b b)$ & $3.89 \times 10^{-3}$ & $3.86 \times 10^{-3}$ & $6.02 \times 10^{-2}$ & $2.17 \times 10^{-3}$ \\
$M_{T}^{b b}$ & $8.65 \times 10^{-3}$ & $7.37 \times 10^{-3}$ & $1.95 \times 10^{-2}$ & $1.46 \times 10^{-2}$ \\
$\Delta \phi(b, b)$ & $5.68 \times 10^{-3}$ & $1.79 \times 10^{-2}$ & $1.25 \times 10^{-2}$ & $3.12 \times 10^{-4}$ \\
$b_{1} p_{T}$ & $4.38 \times 10^{-3}$ & $5.25 \times 10^{-4}$ & $3.83 \times 10^{-4}$ & $2.39 \times 10^{-2}$ \\
$\Delta \eta(b, b)$ & $1.96 \times 10^{-3}$ & $1.40 \times 10^{-2}$ & $1.30 \times 10^{-3}$ & $2.64 \times 10^{-3}$ \\
$b_{2} p_{T}$ & $1.51 \times 10^{-3}$ & $6.39 \times 10^{-4}$ & $3.02 \times 10^{-3}$ & $1.33 \times 10^{-2}$ \\
$\Delta R(b, b)$ & $4.81 \times 10^{-3}$ & $7.61 \times 10^{-3}$ & $2.33 \times 10^{-3}$ & $1.01 \times 10^{-3}$ \\
$\Delta \theta(b, b)$ & $3.93 \times 10^{-4}$ & $1.22 \times 10^{-4}$ & $4.48 \times 10^{-3}$ & $2.84 \times 10^{-3}$ \\
$\Delta \phi\left(b b, \notin_{T}\right)$ & $1.51 \times 10^{-4}$ & $8.72 \times 10^{-4}$ & $5.21 \times 10^{-4}$ & $1.75 \times 10^{-3}$ \\
$b_{2} \eta$ & $2.31 \times 10^{-5}$ & $1.74 \times 10^{-4}$ & $2.51 \times 10^{-3}$ & $3.69 \times 10^{-4}$ \\
$\Delta \theta(\ell, \ell)$ & $1.54 \times 10^{-3}$ & $7.46 \times 10^{-4}$ & $2.75 \times 10^{-4}$ & $4.41 \times 10^{-4}$ \\
$\ell_{2} \eta$ & $8.62 \times 10^{-4}$ & $1.52 \times 10^{-3}$ & $2.89 \times 10^{-5}$ & $4.02 \times 10^{-5}$ \\
$\Delta \phi\left(\mathrm{Z}, \mathbb{E}_{T}\right)$ & $5.32 \times 10^{-5}$ & $1.29 \times 10^{-3}$ & $5.81 \times 10^{-4}$ & $1.59 \times 10^{-4}$ \\
$M_{\ell \ell}$ & $4.81 \times 10^{-4}$ & $9.99 \times 10^{-5}$ & $1.04 \times 10^{-3}$ & $1.22 \times 10^{-4}$ \\
$\Delta \eta(\ell, \ell)$ & $4.54 \times 10^{-4}$ & $5.56 \times 10^{-4}$ & $1.19 \times 10^{-4}$ & $8.19 \times 10^{-5}$ \\
$Z$ vertex & $1.29 \times 10^{-4}$ & $7.92 \times 10^{-4}$ & $3.05 \times 10^{-5}$ & $2.88 \times 10^{-5}$ \\
$Z H p_{x}($ fit $)$ & $1.56 \times 10^{-4}$ & $6.05 \times 10^{-4}$ & $1.32 \times 10^{-4}$ & $8.38 \times 10^{-5}$ \\
$\cos \left(\theta^{*}\right)$ & $7.33 \times 10^{-5}$ & $1.58 \times 10^{-4}$ & $6.56 \times 10^{-4}$ & $4.09 \times 10^{-5}$ \\
$Z H p_{y}($ fit $)$ & $3.10 \times 10^{-4}$ & $3.90 \times 10^{-4}$ & $3.73 \times 10^{-5}$ & $6.36 \times 10^{-5}$ \\
$\eta_{\ell \ell}$ & $2.16 \times 10^{-4}$ & $2.08 \times 10^{-4}$ & $6.02 \times 10^{-5}$ & $2.37 \times 10^{-4}$ \\
$\eta_{b b}$ jets & $5.89 \times 10^{-5}$ & $4.02 \times 10^{-5}$ & $1.21 \times 10^{-4}$ & $2.05 \times 10^{-4}$ \\
\hline \hline & $4.38 \times 10^{-6}$ & $7.03 \times 10^{-5}$ & $3.38 \times 10^{-5}$ & $1.81 \times 10^{-4}$ \\
\hline \hline
\end{tabular}


Table 8.9: The percentage change in the expected and observed $95 \%$ confidence limits for the mu+track channel (combining Run IIa and Run IIb) achieved by using the RF output instead of the di-jet invariant mass. The limits are calculated ignoring systematics.

\begin{tabular}{|c|cc|}
\hline \hline $\begin{array}{c}\text { Higgs Boson } \\
\text { Mass }(\mathrm{GeV})\end{array}$ & $\begin{array}{c}\text { Gain in Expected } \\
\text { Sensitivity (\%) }\end{array}$ & $\begin{array}{c}\text { Gain in Observed } \\
\text { Sensitivity (\%) }\end{array}$ \\
\hline 100 & 8.54 & 0.10 \\
105 & 19.06 & 11.78 \\
110 & 16.68 & -4.86 \\
115 & 20.47 & 25.81 \\
120 & 18.13 & 28.44 \\
125 & 22.79 & 24.51 \\
130 & 20.45 & 20.76 \\
135 & 21.20 & 10.88 \\
140 & 22.59 & 31.12 \\
145 & 23.12 & 24.41 \\
150 & 20.35 & 27.23 \\
\hline \hline
\end{tabular}




\subsection{Systematic Uncertainties}

We now consider the sources of systematic error which affect this search for $Z H$ production.

Luminosity There is a $6 \%$ flat systematic uncertainty associated with the measurement of the luminosity.

Jet Energy Scale (JES) The RF takes as input several variables which depend on measurements of the jets. For this reason, the JES systematic must be treated as a shape. Since we normalize to the $Z$ peak, the shape of the systematic varries around zero, reflecting the changes in the fractional size of the signal and background samples with respect to the whole.

Jet Energy Resolution (JER) The JER is treated identically to the JES by varying the MC smearing by $\pm 1 \sigma$.

Jet Reconstruction Efficiency (Jet Eff) The Jet Eff uncertainty is estimated by repeating the analysis and shifting the jet reconstruction parametrization down by one $\sigma$.

$Z p_{T}$ Modeling The uncertainty on the $Z p_{T}$ re-weighting function is calculated from the covariance matrix of the fit. The error is fluctuated up and down one sigma and the events are re-weighted accordingly.

Lepton Resolution and Trigger Efficiency A flat systematic is quoted for the parametrization of the mu+jets trigger suite. However, we introduce a shape due to the error on the fit of the ratio of the triggered and inclusive datasets.

Since both the standard muon, and the track-only muon rely on the tracking system for their $p_{T}$ measurement, the lepton resolution systematic is identical for both. Although a number of the RF variables depend on lepton measurements, the systematic on the final variable was found to be flat. The lepton systematic is highly correlated with the trigger systematic and the two are merged by adding them in quadrature.

$W W$ and $W Z$ Production Cross Sections The theoretical value of the $W W$ and $W Z$ cross sections has a $7 \%$ uncertainty [41] which is treated as a flat $7 \%$ systematic.

$Z+$ Jets Production Cross Sections The cross section of the $Z+n l p$ process is known to within $10 \%$. The theoretical uncertainties on heavy flavour production are however substantially larger at 30\%. This takes into account the $k$ factors applied to the Alpgen samples in Section 8.1.3.

Multijet Normalization To preserve statistics, the $b$-tagged multijet sample is derived by re-normalizing the sample prior to $b$-tagging. As a result, there is a $25 \%$ systematic uncertainty associated with the multijet yeild. 
AlPGEN Jet Reweighting The proper modelling of the jet distributions in AlPGEN required that we apply a set of corrections on the leading jet $\eta$, second jet $\eta, \Delta \phi(j, j), \Delta \eta(j, j)$, and $\Delta R(j, j)$ distributions. This error on the fitting functions used to derive the corrections determine a shape dependent systematic.

Tag Rate Functions The shape and size of the systematic error on the $b$-tagging functions described in Section 5.7 are determined by independantly fluctuating the light jet and heavy jet TRF, and reweighting the events accordingly.

Taggability Although the taggability functions were applied directly to the MC, the systematic is determined using a reweighting. The uncertainty on the taggability is parametrized as a function of jet $p_{T}$ and $\eta$. 


\subsection{Limits}

The output of the MVA is fed to the ColliE statistical package, described in Chapter 6 . A $95 \%$ confidence level limit is obtained on the production cross section for each of the hypothesized Higgs masses. The results are expressed in units of the scale factor which would have to be applied to the Standard Model cross section. Using a scale factor rather than the cross section itself will allow easier comparisons with channels in which the Higgs is produced via a different production mechanism. The $Z H \rightarrow m u+t r k b b$ results are represented graphically, plotting the expected and observed limits, in figure Figure 8.17 and listed in Table 8.10. A preliminary version of this analysis was combined with the three other $Z H$ channels $(\mu \mu$, ee, and $e+e I C R)$ and presented at the Moriond conference in Aosta, Italy in March of 2009 [55]. A second combination with updated results is underway, but has not been completed as of this writing. The combined limits from March are also shown in figure Figure 8.17 and are listed for each channel in Table 8.11. Not all channels used the full $4.2 \mathrm{fb}^{-1}$ in the preliminary result, and a less powerful multivariate discriminant was in use at the time. Additionally, further studies on the jet resolution functions have improved the performance of the kinematic fit. Finally, in the last five months, we have developed a better understanding of the jet distributions in ALPGEN - which has substantially reduced the size of one of our largest systematic uncertainties.
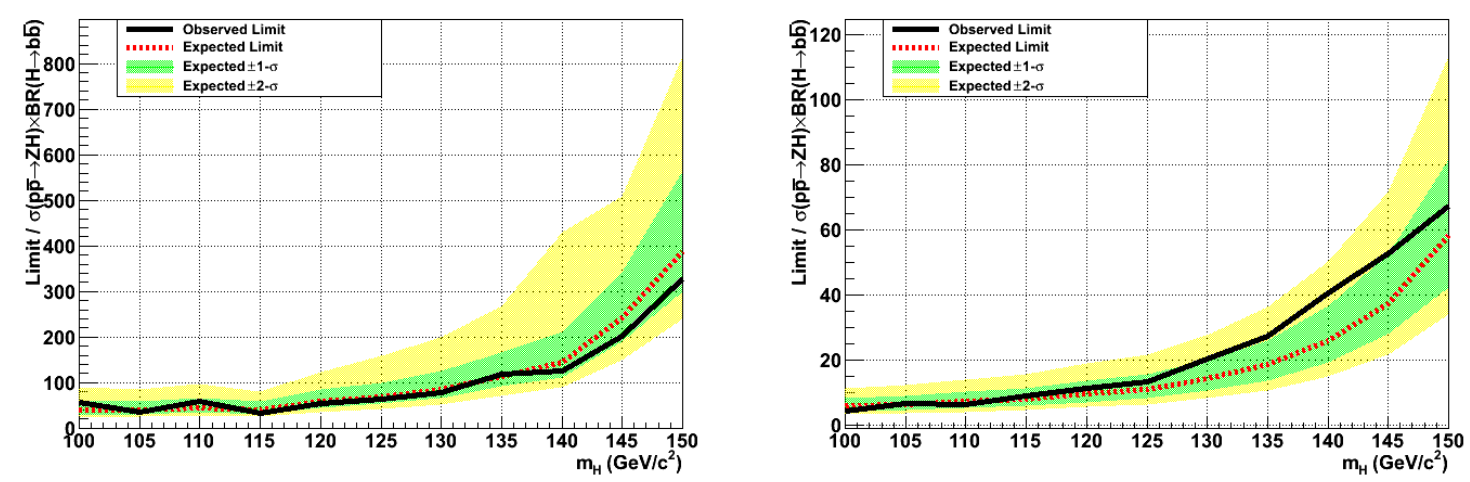

Figure 8.17: The expected and observed 95\% CL limits on the Higgs boson cross section. The limits are reported as a scale factor which would have to be applied to the SM cross section. The latest version of the $m u+t r k$ channel is plotted on the left, the $Z H \rightarrow \ell \ell b b$ combination, as of March 2009, is plotted on the right.

The March results can also be compared to the limits from an earlier $Z H$ analysis performed on $2.3 \mathrm{fb}^{-1}$ in January of 2008, without the benefit of the mu+trk and e+ICR channels [56]. The $2.3 \mathrm{fb}^{-1}$ limit for a Higgs boson with mass $115 \mathrm{GeV}$ was 8.95 times the SM. The March 2009 expected limit of 8.0 represents a $12 \%$ improvement in sensitivity beyond what is expected from simply increasing the size of the dataset. For comparison, Figure 8.18 shows the expected limits for a $115 \mathrm{GeV}$ Higgs boson from scalling the $2.3 \mathrm{fb}-1$ and $4.2 \mathrm{fb}-1$ results by the square-root 
Table 8.10: The expected and observed 95\% CL limits on the Higgs boson cross section from the $m u+t r k$ channel as of this writing. The numbers reported here are the scale factor which would have to be applied to the SM Higgs cross section. !! TO BE FILLED IN !!

\begin{tabular}{|c|c|c|c|}
\hline \hline & Run IIa & Run IIb & Combination \\
\hline$M_{H}(\mathrm{GeV})$ & Exp (Obs)/SM & Exp (Obs)/SM & Exp (Obs)/SM \\
100 & $101(114)$ & $42.2(61.9)$ & $39.5(57.6)$ \\
105 & $86.3(49.1)$ & $51.3(60.9)$ & $40.9(34.8)$ \\
110 & $94.5(100)$ & $58.3(78.8)$ & $45.8(58.5)$ \\
115 & $75.4(52.8)$ & $54.3(58.1)$ & $40.2(33.8)$ \\
120 & $122(101)$ & $74.9(77.7)$ & $58.1(54.7)$ \\
125 & $135(95.2)$ & $88.0(91.2)$ & $69.6(63.2)$ \\
130 & $194(132)$ & $113(149)$ & $85.3(78.3)$ \\
135 & $214(205)$ & $152(163)$ & $115(119)$ \\
140 & $299(179)$ & $190(206)$ & $145(125)$ \\
145 & $481(278)$ & $308(349)$ & $242(201)$ \\
150 & $721(439)$ & $516(629)$ & $387(328)$ \\
\hline \hline
\end{tabular}

Table 8.11: The expected and observed 95\% CL limits on the Higgs boson cross section presented at the Moriond conference in March of 2009. The numbers reported here are the scale factor which would have to be applied to the SM Higgs cross section.

\begin{tabular}{|c|c|c|c|c|c|}
\hline \hline & Run IIb $\mu \mu$ & Run IIb ee & Full Run II $\mu+$ track & Run IIb $e+$ ICR & Combination \\
\hline$M_{H}(\mathrm{GeV})$ & Exp (Obs)/SM & Exp (Obs)/SM & Exp (Obs)/SM & Exp (Obs)/SM & Exp (Obs)/SM \\
\hline 100 & $9.29(7.7)$ & $11.4(18.5)$ & $38.6(43.4)$ & $42.3(38.0)$ & $6.0(4.3)$ \\
105 & $11.1(9.0)$ & $12.6(19.2)$ & $43.2(45.3)$ & $45.6(53.8)$ & $6.4(6.6)$ \\
110 & $12.8(10.9)$ & $14.6(17.0)$ & $46.7(69.7)$ & $50.2(56.3)$ & $7.3(6.5)$ \\
115 & $14.7(13.3)$ & $15.3(18.7)$ & $50.4(78.0)$ & $57.0(68.2)$ & $8.0(9.1)$ \\
120 & $17.7(14.2)$ & $19.2(23.9)$ & $63.4(75.2)$ & $68.8(87.7)$ & $9.9(11.4)$ \\
125 & $20.7(19.5)$ & $20.9(27.0)$ & $74.1(86.7)$ & $85.5(91.2)$ & $11.2(13.5)$ \\
130 & $26.2(25.6)$ & $27.4(42.4)$ & $90.4(120)$ & $102(130)$ & $14.5(20.3)$ \\
135 & $34.2(44.1)$ & $34.9(43.6)$ & $126(142)$ & $146(159)$ & $18.7(27.5)$ \\
140 & $47.9(62.0)$ & $50.3(73.2)$ & $167(188)$ & $198(232)$ & $26.2(40.8)$ \\
145 & $64.9(91.5)$ & $69.2(85.5)$ & $250(252)$ & $269(243)$ & $37.5(52.7)$ \\
150 & $104(129.3)$ & $114(123)$ & $428(331)$ & $457(380)$ & $58.3(67.6)$ \\
\hline \hline
\end{tabular}


of the luminosity.

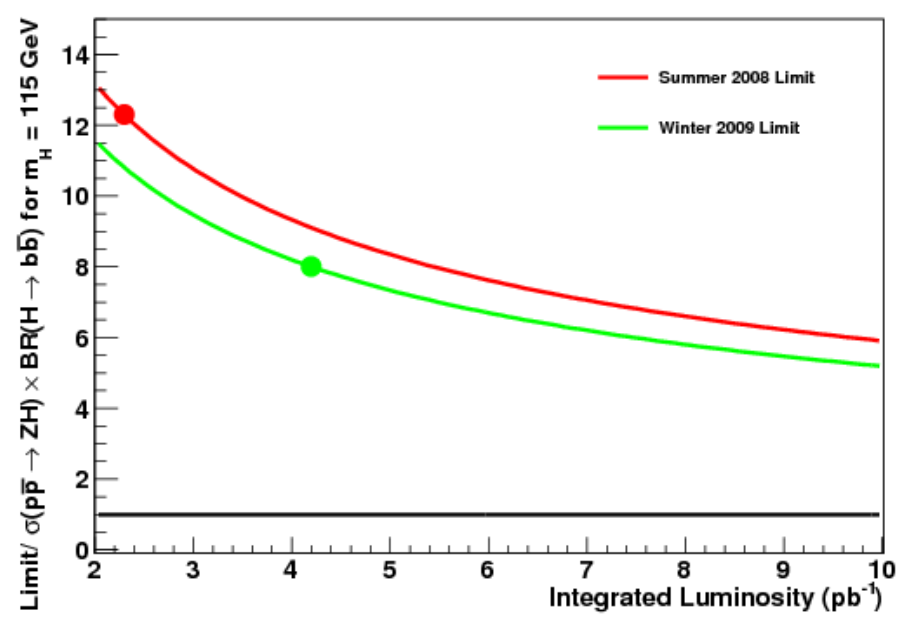

Figure 8.18: The combined $Z H \rightarrow \ell \ell b b$ expected limit for a $115 \mathrm{GeV}$ Higgs as of the Summer of 2008 and the Winter of 2009. The colored lines track the expected limit as a function of $\sqrt{\mathcal{L}}$.

Unfortunately, neither $\mathrm{D} \varnothing$ or CDF currently have the sensitivity necessary to observe the Higgs boson on their own. Direct search results from the two experiments are combined into a single Tevatron result, which was also last updated in March of 2009 and is shown graphically in Figure 8.19 [57]. The Tevatron result contains seventy-five mutually exclusive final states from $H \rightarrow b \bar{b}, H \rightarrow W^{+} W^{-}, H \rightarrow \tau^{+} \tau^{-}$, and $H \rightarrow \gamma \gamma$, where the Higgs has been produced via $g g \rightarrow H, q \bar{q} \rightarrow W / Z H$, or $q \bar{q} \rightarrow q^{\prime} \bar{q}^{\prime} H$. The combination was performed on analyses using betwen 0.9 to $4.2 \mathrm{fb}^{-1}$ of data. The current expected limit on a $115 \mathrm{GeV}$ mass SM Higgs is 2.4 times the standard model, whereas a mass lying in the $160<m_{H}<170 \mathrm{GeV}$ window has been excluded at the 95\% CL. 


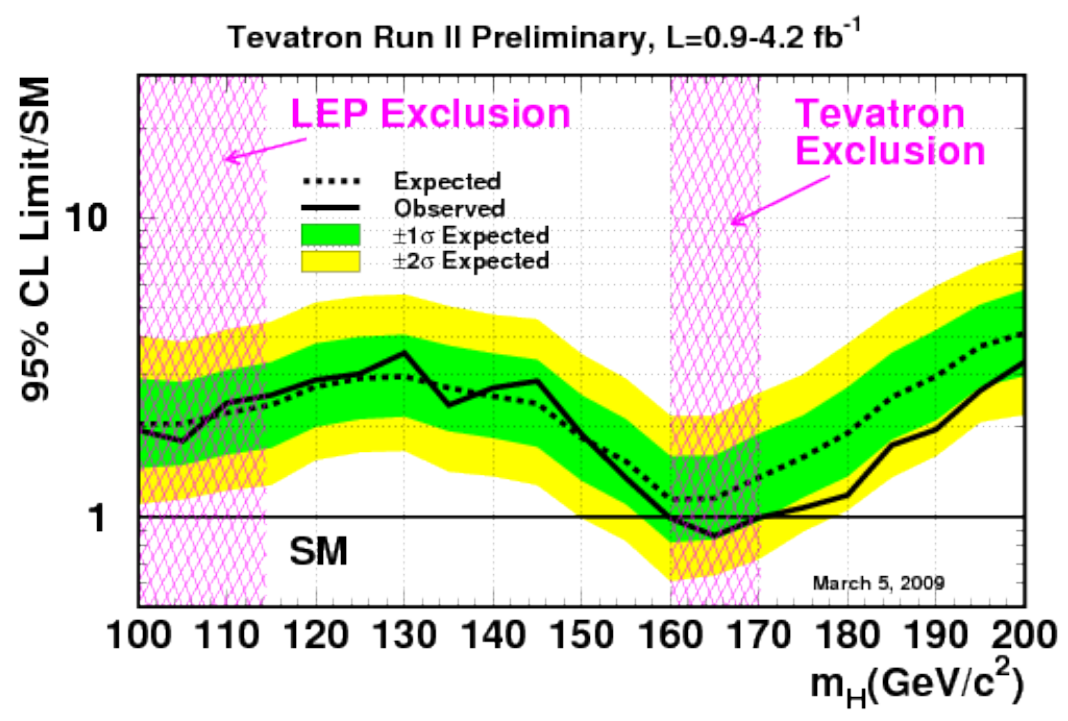

Figure 8.19: The Tevatron combination 95\% CL on the cross section of the SM Higgs as of March 2009. The dashed (solid) black line shows the expected (observed) value and the green (yellow) area shows the 1 (2) sigma band. 


\section{Chapter 9}

\section{Conclusion and Outlook}

"Ah? Inventer ce n'est pas travailler?!?! Pffouh... Avec des idées pareilles on en serait encore á faire le travail de bureau au silex taillé... Comme les hommes de cro-magnon!!"

We have presented two results from the $\mathrm{D} \varnothing$ experiment. The search for $Z Z$ di-boson production demonstrated our ability to model, search, and most importantly, measure a signal with a cross section of $1.4 \mathrm{pb}$ in the face of large backgrounds. The search proved fruitful when the results from the $Z Z \rightarrow \ell \ell \nu \nu$ and $Z Z \rightarrow \ell \ell \ell^{\prime} \ell^{\prime}$ channels were combined, resulting in a five sigma observation in good agreement with the Standard Model. This observation of $Z Z$ production was the first at a Hadron collider. No hint of new physics from $Z Z Z$ or $Z Z \gamma$ anomalous trilinear gauge couplings was observed.

The search for $Z H$ production demonstrated ways in which we are expanding the coverage of our analyses by considering channels with looser, or entirely new, selection requirements. The inclusion of track-only muons increased our yields by $15 \%$. To put things into perspective, over $4.2 \mathrm{fb}^{-1}$ this is the equivalent of collecting data for an additional 12 weeks at our best delivered instantaneous luminosity, albeit at a substantially reduced financial cost. The kinematic fit further improved the di-jet invariant mass resolution and results in an additional $8 \%$ improvement in sensitivity. After cutting on the fitter $\chi^{2}$, the $m u+t r k$ and traditional di-muon analyses have similar sensitivity per unit of yield. The mu+trk, di-muon, e+ICR, and di-electron channels have been combined with the other available D $\varnothing$ Higgs searches and they are expected to be included in the upcoming updated Tevatron combination.

This is most certainly not the final word on either of these two analyses. Over $6 \mathrm{fb}^{-1}$ of data have now been collected with the $\mathrm{D} \emptyset$ detector. This, combined with the additional acceptance that track-only muons could provide, means that there is approximately a factor of 
2.5 more data to be analyzed for the $Z Z$ analysis. Scaling the sensitivity of the $Z Z \rightarrow \ell \ell \nu \nu$ analysis by the square-root of the increase in dataset size indicates that we would now be able to make a greater than 3 sigma observation in this channel alone. If a 3 sigma observation is currently plausible in the $Z Z \rightarrow \ell \ell \nu \nu$ channel, it is not hard to imagine that 3 sigma evidence, or exclusion, of the Higgs boson at a mass range above $135 \mathrm{GeV}$ will be possible with the full $10 \mathrm{fb}^{-1}$ dataset.

Since the $160<m_{H}<170 \mathrm{GeV}$ region of phase space has already been excluded by combining results from $\mathrm{CDF}$ and $\mathrm{D} \emptyset$, a single-channel exclusion would be a technical achievement and a point of pride for the collaboration, but the real region of interest is around a Higgs boson mass of $115 \mathrm{GeV}$. Here, a single-channel observation or exclusion is very unlikely. The latest Tevatron combination places a 95\% C.L. limit on a cross section 2.4 times the standard model for a $115 \mathrm{GeV}$ Higgs (See Figure 8.19) using up to $4.2 \mathrm{fb}^{-1}$ of data. Since not all channels used the same amount of luminosity, it makes sense to talk in terms of a sensitivity-weighted average of the luminosity. For a $115 \mathrm{GeV}$ mass Higgs boson, the sensitivity-weighted average analyzed luminosity is only $2.55 \mathrm{fb}^{-1}$. Scaling this limit to a $10 \mathrm{fb}^{-1}$ dataset, the Tevatron combination is expected to be only $\sim 20 \%$ shy of excluding a SM Higgs at $115 \mathrm{GeV}$ at $95 \%$ CL.

The improvement attained in the $Z H \rightarrow \ell \ell b b$ analysis over the last five months is an excellent example of the type of performance we can continue to expect. There are a number of projects aimed at further improving our sensitivity already underway, and more which have yet to be thought up. With this in mind, it is possible, and even quite likely, that the Tevatron may yet achieve an additional $20 \%$ gain in sensitivity, if not more. The Large Hadron Collider, which is expected to begin taking data imminently and will probe particle collisions at much higher energies, is expected to become sensitive to a low mass Higgs within two to three years. The competition surrounding searches for the Higgs Boson will make the coming years a very exciting time to be working in Particle Physics. 


\section{Bibliography}

[1] P. Langacker, "Introduction to the Standard Model and Electroweak Physics," arXiv:0901.0241 [hep-ph].

[2] A. Pich, "The Standard model of electroweak interactions," arXiv:0705.4264 [hep-ph].

[3] Fermilab Visual Media Services. Website. http://www-visualmedia.fnal.gov/VMS_Site_2/index.shtml.

[4] J. Thompson, "Introduction to Colliding Beams at Fermilab.,". FERMILAB-TM-1909.

[5] D. Möhl, G. Petrucci, L. Thorndahl, and S. van der Meer Phys. Rep. C58 (1980) 73-119.

[6] The DØ Collaboration, S. Abachi et al., "The D0 Detector," Nucl. Instrum. Meth. A338 (1994) 185-253.

[7] The DØ Collaboration, V.M. Abazov et al., "The Upgraded DØ Detector," Nucl. Inst. Meth. A 565 (2006) 463, arXiv:physics/0507191.

[8] The DØ Collaboration, T. L. Edwards et al., "Determination of the effective inelastic $p \bar{p}$ cross-section for the D0 Run II luminosity measurement,". FERMILAB-TM-2278-E.

[9] C. Daly, L. Lubatti, W. Kuykendall, and T. Wang, C.J. T. Zhao, "The Design of the Layer-0 Silicon Detector for the D-Zero Experiment," IEEE (2004) .

[10] The DØ Collaboration, V. M. Abazov et al., "Run IIb Upgrade Technical Design Report,". FERMILAB-PUB-02-327-E.

[11] T. Sjostrand, S. Mrenna, and P. Skands, "Pythia6.4 Physics and Manual," JHEP 05 (2006) 026, arXiv:hep-ph/0603175.

[12] M. L. Mangano, M. Moretti, F. Piccinini, R. Pittau, and A. D. Polosa, "ALPGEN, a generator for hard multiparton processes in hadronic collisions," JHEP 07 (2003) 001, arXiv:hep-ph/0206293.

[13] J. Pumplin et al., "New generation of parton distributions with uncertainties from global QCD analysis," JHEP 07 (2002) 012, arXiv:hep-ph/0201195. 
[14] S. H oche et al., "Matching Parton Showers and Matrix Elements," arXiv:0602031 [hep-ph].

[15] Y. Fisyak and J. Womersley, "D0gstar (DØ GEANT Simulation of the Total Apparatus Response," DØ Note 3191 .

[16] R. Brun and F. Carminati, "CERN Program Library Writeup W5013,".

[17] Q. Li and S. Protopopescu, "D0sim,". http://www-d0.fnal.gov/computing/MonteCarlo/simulation/d0sim.html.

[18] H. Greenlee, "The DØ Kalman Track Fit," DØ Notes 4303 .

[19] A. Khanov, "HTF: histogramming method for finding tracks. The algorithm description," DØ Notes 3778 .

[20] G. Borissov, "Ordering a Chaos or... Technical Details of AA Tracking,". www-d0.fnal.gov/global_tracking/talks/20030228/talk-adm-030228.ps.

[21] A. Schwartzman and C. Tully, "Primary Vertex Reconstruction by Means of Adaptive Vertex Fitting," DØ Notes 4918 .

[22] A. Schwartzman and M. Narain, "Probabilistic Primary Vertex Selection," DØ Notes 4042 .

[23] P. Calfayan, T. Gadfort, G. Hesketh, V. Lesne, M. Owen, R. Ströhmer, V. Shary, and B. Tuchming, "Muon Identification Certification for p17 data," DØ Notes 5157.

[24] F. Fleuret, "The DØ Electron/Photon Analysis Package EMAnalyze," DØ Notes 3888 .

[25] D. Chapin, H. Fox, J. Gardner, R. Illingworth, A. Lyon, and J. Zhu, "Measurement of $Z \rightarrow e^{+} e^{-}$and $W \rightarrow e^{ \pm} \nu$ Production Cross Sections Using One Tight Central Electron," DØ Notes 4897 .

[26] T. Andeen, J. Hays, and H. Schellman, "Electron Level Energy Correction in DØ Reconstruction p14 pass 2," DØ Notes 5104.

[27] L. Wang, J. Hays, C. Schwanenberger, and J. Mitrevski, "Electron Likelihood Efficiency in p17," DØ Notes $\mathbf{5 1 1 4}$.

[28] The DØ Collaboration, V.M. Abazov et al., "b-jet Identification in the DØ Experiment," Nucl. Instrum. Meth. A . (to be submitted).

[29] E. Berger, ed., Research directions for the decade, Snowmass. Singapore: World Scientific, June 25 - July 13, 1990. 
[30] R. Fr uhwirth Nucl. Inst. Meth. A 262 (1987) 444.

[31] B. Clement, "SystemD or how to get signal, backgrounds and their efficiencies with real data," DØ Note $\mathbf{4 1 5 9}$.

[32] Particle Data Group , C. Amsler et al., "Review of Particle Physics," Phys. Lett. B667 (2008) 1.

[33] W. Fisher, "Collie: A Confidence Level Limit Evaluator," DØ Note 5595.

[34] The DØ Collaboration, V.M. Abazov et al., "Observation of $Z Z$ Production in $p \bar{b}$ Collisions at $\sqrt{s}=1.96 \mathrm{TeV}, "$ Phys. Rev. Lett. 101 (2008) 171803, arXiv:0808.0703 [hep-ex].

[35] S. Protopopescu and P. Svoisky, " $\tau$ Identification with Neural Networks for p17 Data," DØ Note $\mathbf{5 0 9 4}$.

[36] U. Baur, T. Han, and J. Ohnemus, "QCD Corrections and Anomalous Couplings in $Z \gamma$ Production at Hadron Colliders," Phys. Rev. D57 (1998) 2823, arXiv:hep-ph/9710416.

[37] A. Askew, A. Lyon, and G. Pawloski, "Measurement of the Charge Signed Photon-Lepton Rapidity Difference in W $\gamma$ Events," DØ Note 5256.

[38] E. Barberis, T. Golling, I. Iashvili, A. Juste, A. Quadt, and P. Schieferdecker, "The Matrix Method and its Error Calculation," DØ Note 4564.

[39] A. Aguiló, P. Baringer, A. Bean, C. Belanger-Champagne, J. Benitez, and E. Boos, "Search for Single Top Quark Production in 1 fb-1 of Data," DØ Note 5285 .

[40] The OPAL Collaboration, K. Ackerstaff et al., "Search for Anomalous Production of Di-lepton Events with Missing Transverse Momentum in $e^{+} e^{-}$Collisions at sqrt(s) $=161$ and 172 GeV," Eur. Phys. J C4 (1998) 47, arXiv:hep-ex/9710010.

[41] J. M. Campbell and R. K. Ellis, "An update on vector pair production at hadron colliders," Phys. Rev. D60 (1999) 113006, arXiv:hep-ph/9905386.

[42] P. W. Higgs, "Broken symmetries, massless particles and gauge fields," Phys. Lett. 12 (1964) 132-133.

[43] The LEP Electroweak Working Group, D. Abbaneo et al. Website. http://lepewwg. web.cern.ch/LEPEWWG/.

[44] The TeV4LHC Higgs Working Group . Website. http://maltoni.home.cern.ch/maltoni/TeV4LHC/. 
[45] Y. Peters, M. Begel, E. Shabalina, and D. Wicke, "Study of the $W+$ jets heavy flavor scale factor in p17," DØ Note $\mathbf{5 4 0 6}$.

[46] J.-F. Grivaz, "K, K', Scale factors, and All That,". http://www-do.hef.kun.nl/ /askArchive .php?base=agenda\&categ=a08266\&id=a08266s1t4/transparencies.

[47] D. Price, "Alpgen Jet Reweighting Factors,". http://www-d0.hef .kun.nl/ /askArchive. php?base=agenda\&categ=a09896\&id=a09896s 1 t7 / transparencies .

[48] M. Rangel, "Vertex Confirmation SF For Jets,". http://www-do.hef.kun.nl/ /askArchive $\cdot$ php?base=agenda\&categ=a09320\&id=a09320s1t1/transparencies.

[49] S. Calvet, "Taggability SF in p17,". http://www-d0.hef.kun.nl//askArchive.php? base=agenda\&categ=a09320\&id=a09320s1t2/transparencies.

[50] S. Calvet, "p20 Taggability SF,". http://www-d0.hef.kun.nl//askArchive.php?base= agenda\&categ=a09896\&id=a09896s1t9/transparencies.

[51] W. H. Press, S. A. Teukolsky, W. T. Vetterling, and B. P. Flannery, Numerical Recipes: The Art of Scientific Computing. Cambridge University Press, 2007.

[52] Leo Breiman, "Random Forests," Machine Learning 45 (2001) 5-32.

[53] A. Hoecker and P. Speckmayer and J. Stelzer and J. Therhaag and E. von Toerne and H. Voss, "TMVA 4 Users Guide," POSACAT 040 (2007), arXiv:physics/0703039.

[54] S. J. Parke and S. Veseli, "Distinguishing $W H$ and $W b \bar{b}$ production at the Fermilab Tevatron," Phys. Rev. D60 (1999) 093003, arXiv:hep-ph/9903231.

[55] L. Ancu, J. BackusMayes, B. Calpas, K. Chan, S. Desai, M. Kirby, M. Mulhearn, E. Nagy, E. Strauss, and T. Yasuda, "Search for $Z H \rightarrow e e b b$ and $Z H \rightarrow \mu \mu b b$ Production in $4.1 \mathrm{fb}^{-1}$ of Run II data with the DZero Detector in $p \bar{p}$ Collisions at $\sqrt{s}=1.96 \mathrm{TeV}$," DØ Notes $\mathbf{5 8 7 6}$.

[56] Taka Yasuda and Others, "Combined cross section limits on ZH production with $2.3 \mathrm{fb}^{-1}$ of Run II data," DØ Notes $\mathbf{5 5 7 0}$.

[57] The DØ and CDF Collaborations , "Combined CDF and DZero Upper Limits on Standard Model Higgs-Boson Production with up to 4.2 fb-1 of Data," arXiv:0903.4001 [hep-ex]. 


\section{Appendix A}

\section{Lepton Fake Rate}

The number of events in which QCD background fakes an electron is estimated using the Matrix Method, as described in [38]. This method relies on four quantities: the number of events passing the "loose" and "tight" selection criteria $\left(N_{l}\right.$ and $\left.N_{t}\right)$, and the probabilities for a real lepton and a fake lepton to pass the "tight" isolation requirements $\left(\varepsilon_{\text {real }}\right.$ and $\left.\varepsilon_{\text {fake }}\right)$.

\section{A.1 Electron Isolation}

We measure the electron $\varepsilon_{\text {fake }}$ by selecting a data sample dominated by QCD background. This is done by requiring that $\mathbb{E}_{T}<10 \mathrm{GeV}$ to reduce the contribution from $\mathrm{W} \rightarrow \mathrm{e} \nu$ and requiring that there be only one EM object to reduce the contributions from $\mathrm{Z} \rightarrow \mathrm{ee}$. All other selection requirements are identical to the analysis "loose" and "tight" cuts. Despite these cuts, there remains a discernible amount of $\mathrm{W}$ and $\mathrm{Z}$ events. This is modeled by $\mathrm{MC}$ which are used to subtract out the remnant.

We take the ratio of "tight" to "loose" electrons as the measurement of $\varepsilon_{\text {fake }}$, assuming that the rate in this QCD enriched region is the same as elsewhere. We do see a dependence on $p_{T}$, number of jets in the event, and location of electrons in the CC or EC regions. However, due to the sparse statistics available for the matrix method after the instrumental background reduction, we fit $\varepsilon_{\text {fake }}$ to a constant for the $\mathrm{CC}+\mathrm{EC}$ regions for the case of 1 or 2 jets and electrons with $p_{T}>20 \mathrm{GeV}$.

\begin{tabular}{|c|c|c|c|c|c|c|c|c|}
\hline \multirow[b]{2}{*}{ Region } & \multicolumn{4}{|c|}{ RunIIa } & \multicolumn{4}{|c|}{ RunIIb } \\
\hline & 1 jet & $\begin{array}{l}\varepsilon_{\text {fake }} \\
2 \text { jets }\end{array}$ & 1 or 2 jets & $\varepsilon_{\text {real }}$ & 1 jet & $\begin{array}{l}\varepsilon_{\text {fake }} \\
2 \text { jets }\end{array}$ & 1 or 2 jets & $\varepsilon_{\text {real }}$ \\
\hline$\overline{\mathrm{CC}}$ & $1.15 \pm 0.05$ & $0.105 \pm 0.05$ & $0.113 \pm 0.05$ & $0.951 \pm 0.002$ & $\overline{\mathrm{X}}$ & $\bar{X}$ & $\bar{X}$ & $0.932 \pm 0.003$ \\
\hline $\mathrm{EC}$ & $0.127 \pm 0.06$ & $0.103 \pm 0.04$ & $0.123 \pm 0.06$ & $0.944 \pm 0.004$ & $\mathrm{X}$ & $\mathrm{X}$ & $\mathrm{X}$ & $0.881 \pm 0.01$ \\
\hline $\mathrm{CC}+\mathrm{EC}$ & $0.119 \pm 0.06$ & $0.104 \pm 0.05$ & $0.115 \pm 0.05$ & $0.950 \pm 0.002$ & $0.134 \pm 0.04$ & $0.127 \pm 0.04$ & $0.131 \pm 0.03$ & $0.928 \pm 0.003$ \\
\hline
\end{tabular}

Table A.1: $\varepsilon_{\text {fake }}$ and $\varepsilon_{\text {real }}$ for RunIIa and RunIIb electrons 

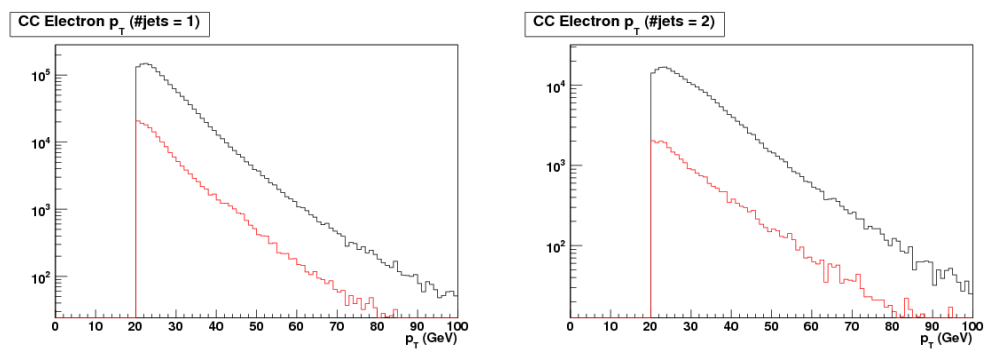

Figure A.1: RunIIa: $p_{T}$ distribution for electrons in the $\mathrm{CC}$ region with 1 (left) and 2 (right) jets in the event
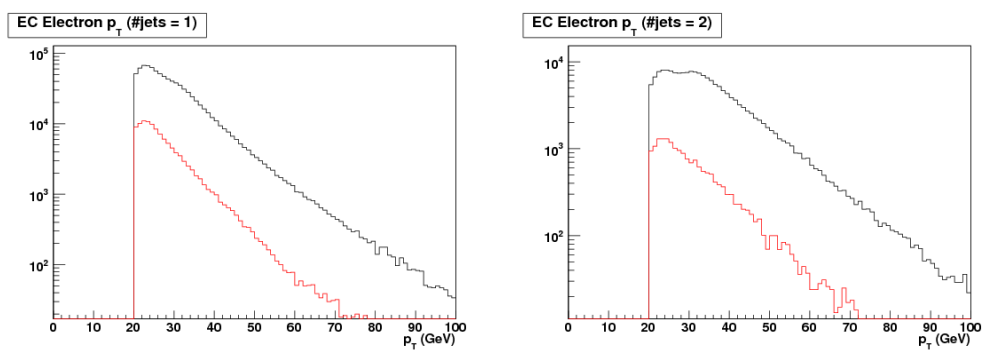

Figure A.2: RunIIa: $p_{T}$ distribution for electrons in the EC region with 1 (left) and 2 (right) jets in the event
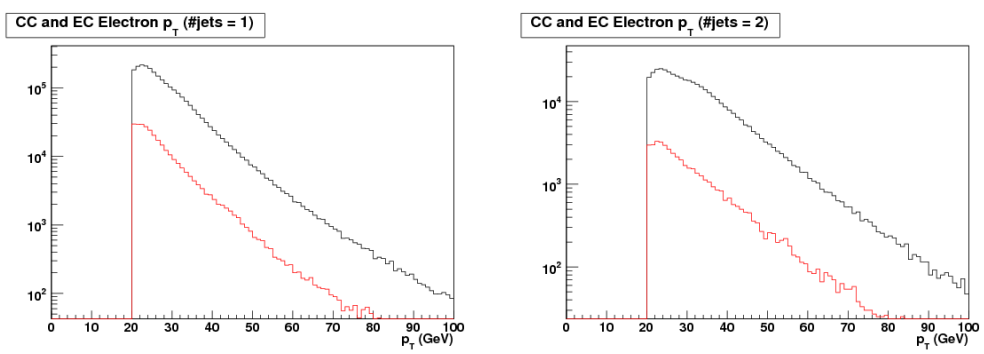

Figure A.3: RunIIa: $p_{T}$ distribution for electrons in the $\mathrm{CC}$ and $\mathrm{EC}$ regions with 1 (left) and 2 (right) jets in the event

The object identification efficiencies for the "loose" and "tight" samples have already been calculated on inclusive $Z \rightarrow e e$ data. Rather than repeating the measurements, $\varepsilon_{\text {real }}$ is determined by taking the ratio of these two efficiencies. The shape of the efficiencies can be seen in Figure A.9 and Figure A.10. 

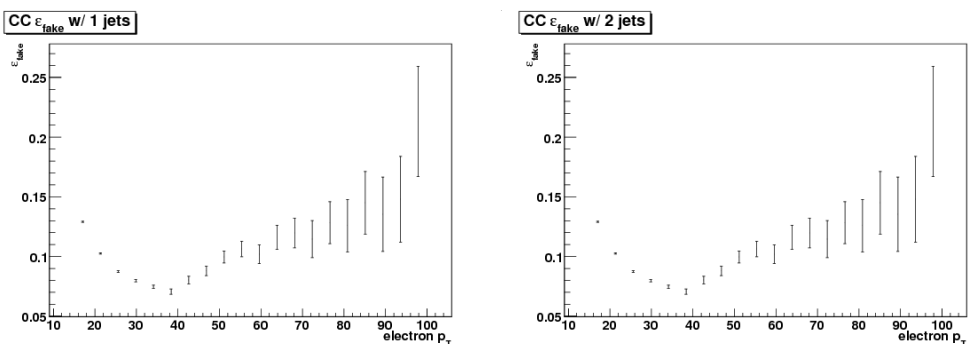

Figure A.4: RunIIa: $\varepsilon_{\text {fake }}$ distribution for electrons in the $\mathrm{CC}$ region with 1 (left) and 2 (right) jets in the event
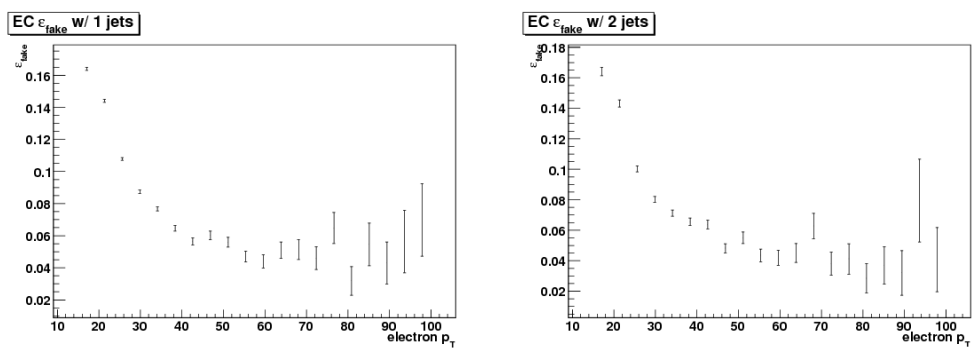

Figure A.5: RunIIa: $\varepsilon_{\text {fake }}$ distribution for electrons in the EC region with 1 (left) and 2 (right) jets in the event
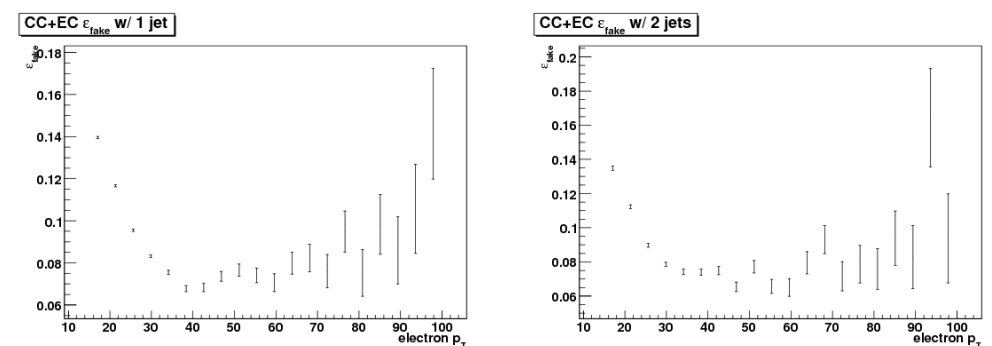

Figure A.6: RunIIa: $\varepsilon_{\text {fake }}$ distribution for electrons in the $\mathrm{CC}$ and EC regions with 1 (left) and 2 (right) jets in the event

\section{A.2 Muon Isolation ${ }^{1}$}

Muons are typically faked by one of two methods. In the first case, hadronic particles may be seen in the muon detector after "punching through" the calorimeter, faking a muon ID. In the second case, genuine muons from QCD may fake the isolation requirements, erroneously passing the selection cuts. We use a method similar to the one outlined in the previous section to estimate the rate at which these two processes occur.

\footnotetext{
${ }^{1}$ Although reported here, the muon fake rate estimation was performed by Gavin Hesketh.
} 

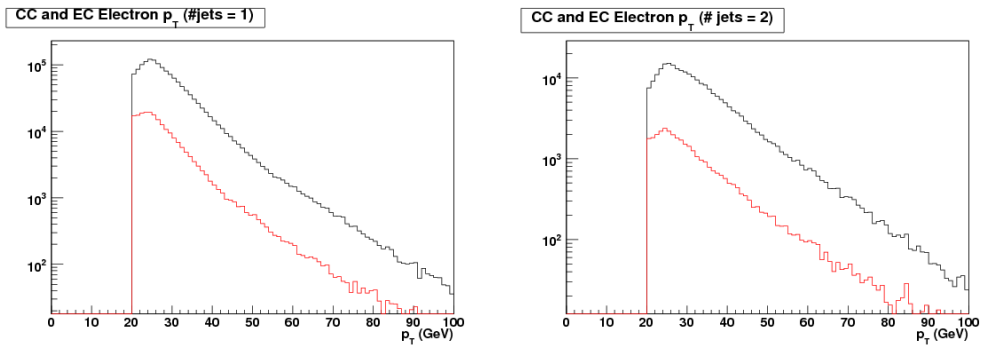

Figure A.7: RunIIb: $p_{T}$ distribution for electrons in the $\mathrm{CC}$ and $\mathrm{EC}$ regions with 1 (left) and 2 (right) jets in the event
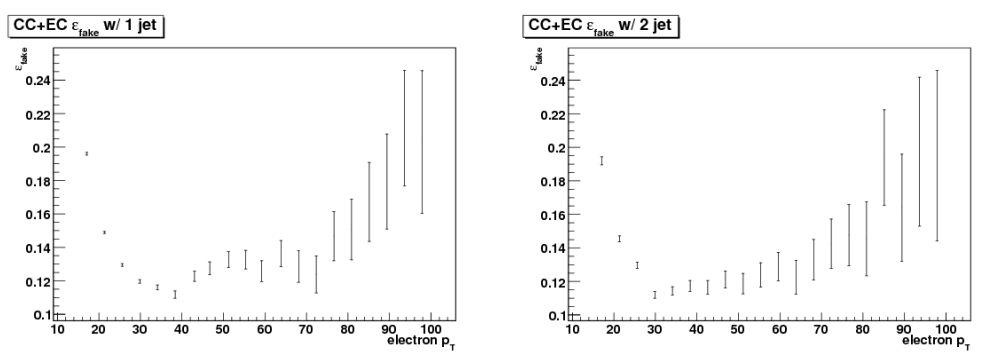

Figure A.8: RunIIb: $\varepsilon_{\text {fake }}$ distribution for electrons in the CC and EC regions with 1 (left) and 2 (right) jets in the event
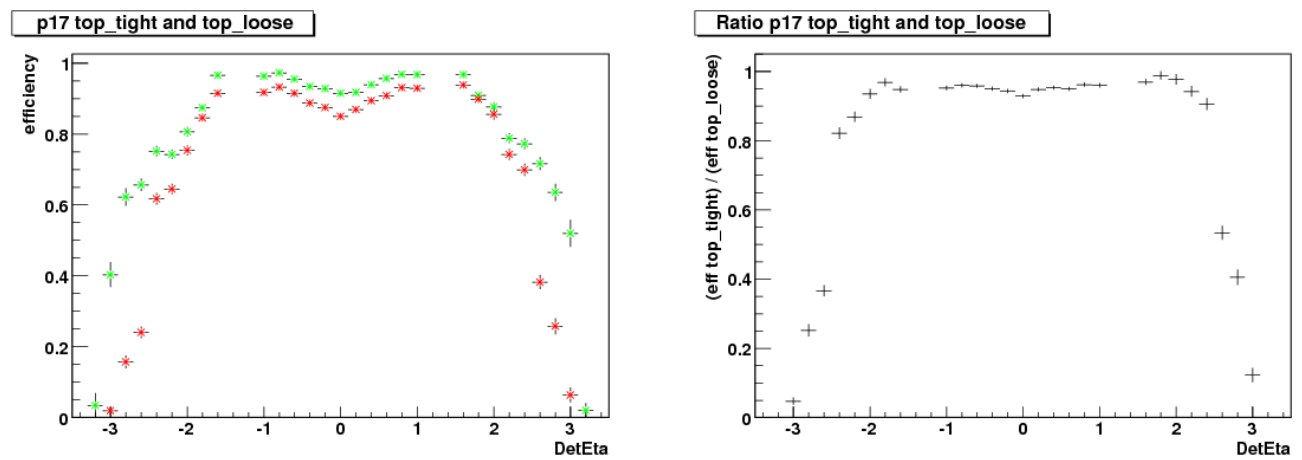

Figure A.9: RunIIa efficiency of the top_tight (in red) and top_loose (in green) electron ids on the left and their ratio on the right.

The estimate is made on a data sample selected by using a suite of jet triggers (the Common Sample Group's QCD skim). The events are required to have at least one muon which satisfies the following cuts. Muons which satisfy these requirements are refered to as "loose":

- $p_{T}>5 \mathrm{GeV}$

- Loose muon quality 

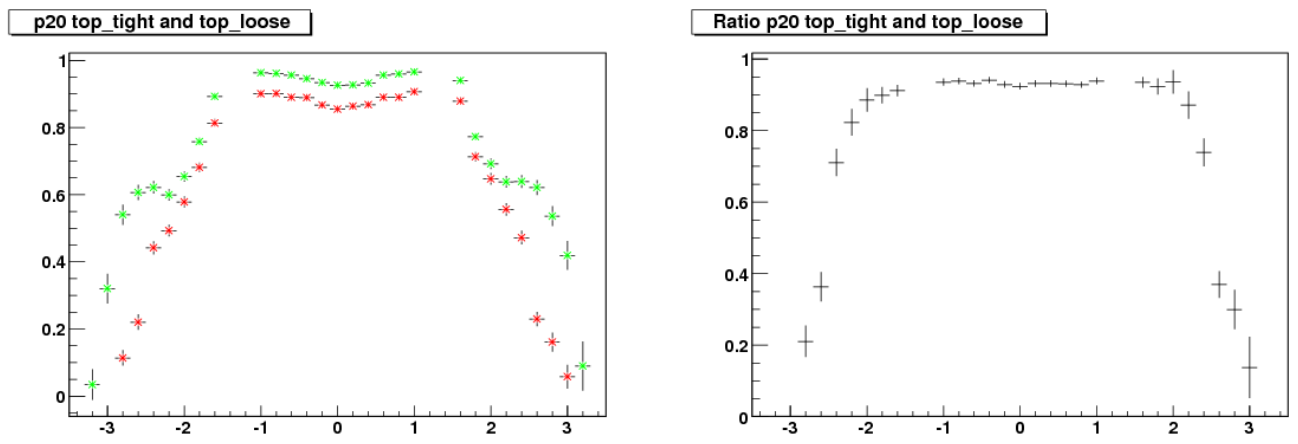

Figure A.10: RunIIb efficiency of the top_tight (in red) and top_loose (in green) electron ids on the left and their ratio on the right.

- Matched to a track with at least $1 \mathrm{SMT}$ hit $D C A<0.02 \mathrm{~cm}$

- The associated track must have $\chi^{2} / d o f<4.0$ (RunIIb only)

We then measure the rate at which these looser muons pass the isolation requirements defined in Section 7.2. The rate is calculated as a function of muon $p_{T}$. The RunIIa and RunIIb functions can be seen in Figure A.11 and Figure A.12. $\varepsilon_{\text {fake }}$, the muon rate at which fakes are passed, is evaluated at $40 \mathrm{GeV}$; the slope $p_{T}$ dependence is used to determine the uncertainty on this value. The same method is used to estimate $\varepsilon_{\text {real }}$, the rate at which real muons are passed, in a sample of $Z$ events in data. The results are summarized in Table A.2

\begin{tabular}{|c|c|c|c|}
\hline \hline \multicolumn{2}{|c|}{ RunIIa } & \multicolumn{2}{c|}{ RunIIb } \\
\hline$\varepsilon_{\text {fake }}$ & $\varepsilon_{\text {real }}$ & $\varepsilon_{\text {fake }}$ & $\varepsilon_{\text {real }}$ \\
\hline \hline $0.969 \pm 0.004$ & $0.045 \pm 0.03$ & $0.896 \pm 0.004$ & $0.035 \pm 0.03$ \\
\hline \hline
\end{tabular}

Table A.2: $\varepsilon_{\text {fake }}$ and $\varepsilon_{\text {real }}$ for RunIIa and RunIIb muons 


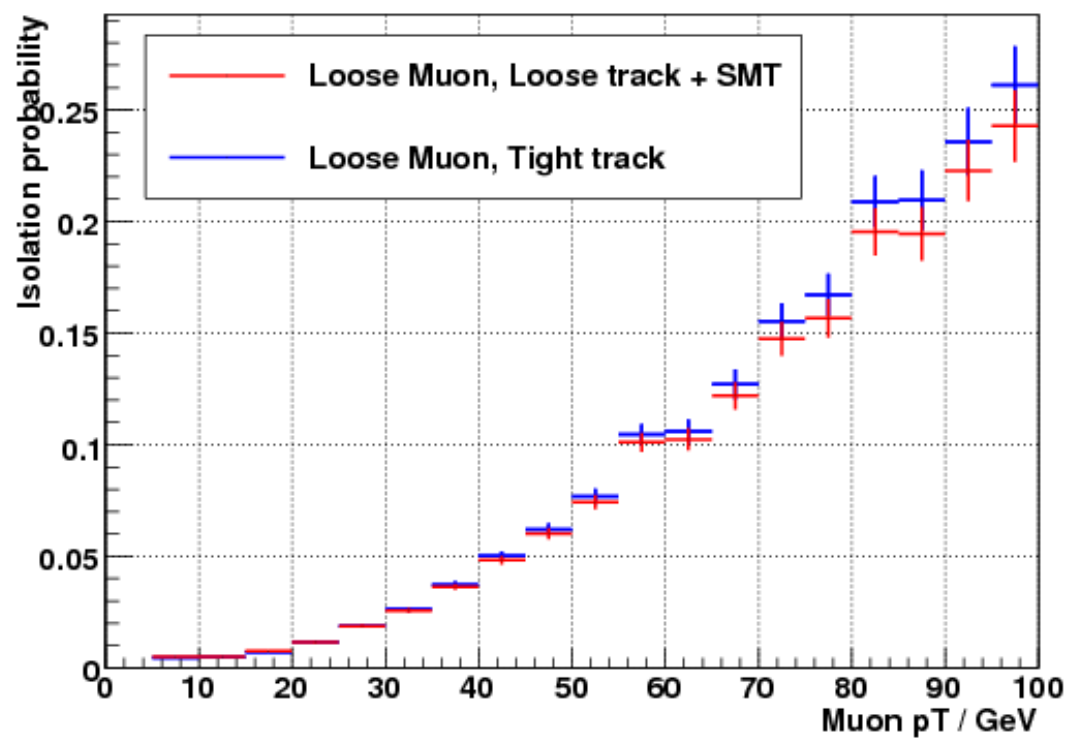

Figure A.11: $p_{T}$ distribution of the muon isolation probability (fake rate) on RunIIa (p17) data, for both the p17 and the p20 "loose" selection criteria.

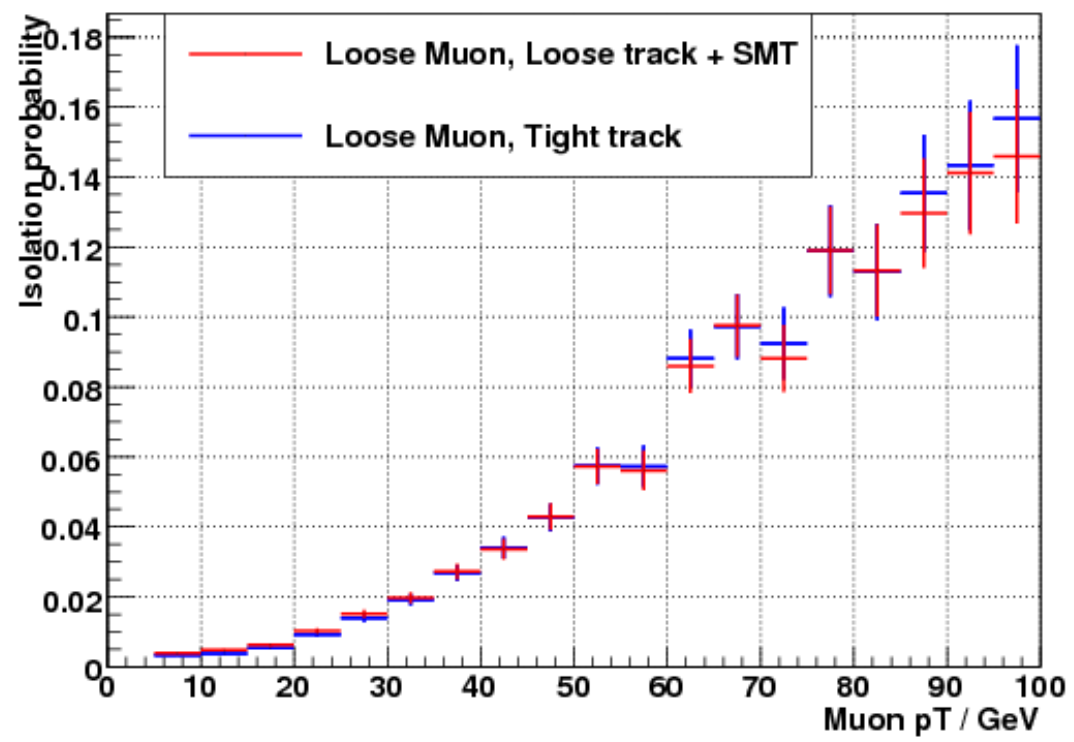

Figure A.12: $p_{T}$ distribution of the muon isolation probability (fake rate) on RunIIb (p20) data, for both the p17 and the p20 "loose" selection criteria. 


\section{Appendix B}

\section{Resolution Functions}

\section{B.1 Muon $p_{T}$ Uncertainty}

It is found that, for the muons, the uncertainty on the lepton $p_{T}$ is poorly modeled by the Monte Carlo. This quantity is used in the $Z Z$ analysis to correct the value of the di-lepton missing $p_{T}$ (See Section 7.4.4) and in the $Z H$ analysis when re-fitting the lepton and jet kinematic values (See Section 8.4). As a solution to this problem we use a transfer function, the output of which tells us the probability that the generated $q / p_{T_{\text {truth }}}$ is reconstructed as $q / p_{T_{\text {reco }}}$ after the appropriate muon smearing has been applied.

$$
\Delta \frac{q}{p_{T}}=\frac{q}{p_{T_{\text {truth }}}}-\frac{q}{p_{T_{\text {reco }}}}
$$

$\Delta \frac{q}{p_{T}}$ is calculated in ten bins of $|\eta|$ from 0.0 to 2.0 and ten bins of $1 / p_{T}$ from 0.0 to 0.6 $\mathrm{GeV}^{-1}$. These are then fit using a Gaussian function. The Gaussian's width $(\sigma)$ is the measure of the muon resolution and we parametrize it as a function of $q / p_{T}$ and $\eta$. Since the muon resolution is in large part determined by the tracking system, we use the detector $\eta$ at the CFT layer, covering muons with $|\eta|<2.0$ and $0.0<1 / p_{T}<0.6 \mathrm{GeV}^{-1}$. The width of the Gaussian is found to be close to constant below $\eta$ of 1.3 (See Figure B.1 and Figure B.2). So we use the following:

$$
\sigma=\left\{\begin{array}{cl}
\sigma_{0}+\frac{\sigma_{1}}{p_{T}} & \left|\eta_{0}\right| \leq 1.28 \\
\sqrt{\left(\sigma_{0}+\frac{\sigma_{1}}{p_{T}}\right)^{2}+\left(\left(c_{0}+\frac{c_{1}}{p_{T}}\right) *\left(|\eta|-\left|\eta_{0}\right|\right)\right)^{2}} & \left|\eta_{0}\right|>1.28
\end{array}\right.
$$

A cutoff of 1.28 is used instead of 1.3 as a result of the binning. The parameters $c_{0}, c_{1}$, $\sigma_{0}$, and $\sigma_{1}$ are determined separately for the RunIIb dataset, the RunIIa dataset before the 2005 shutdown, and the RunIIa dataset after the 2005 shutdown. Since muons without SMT hits on the track have poorer resolution, we further separate the parameters for those with and 

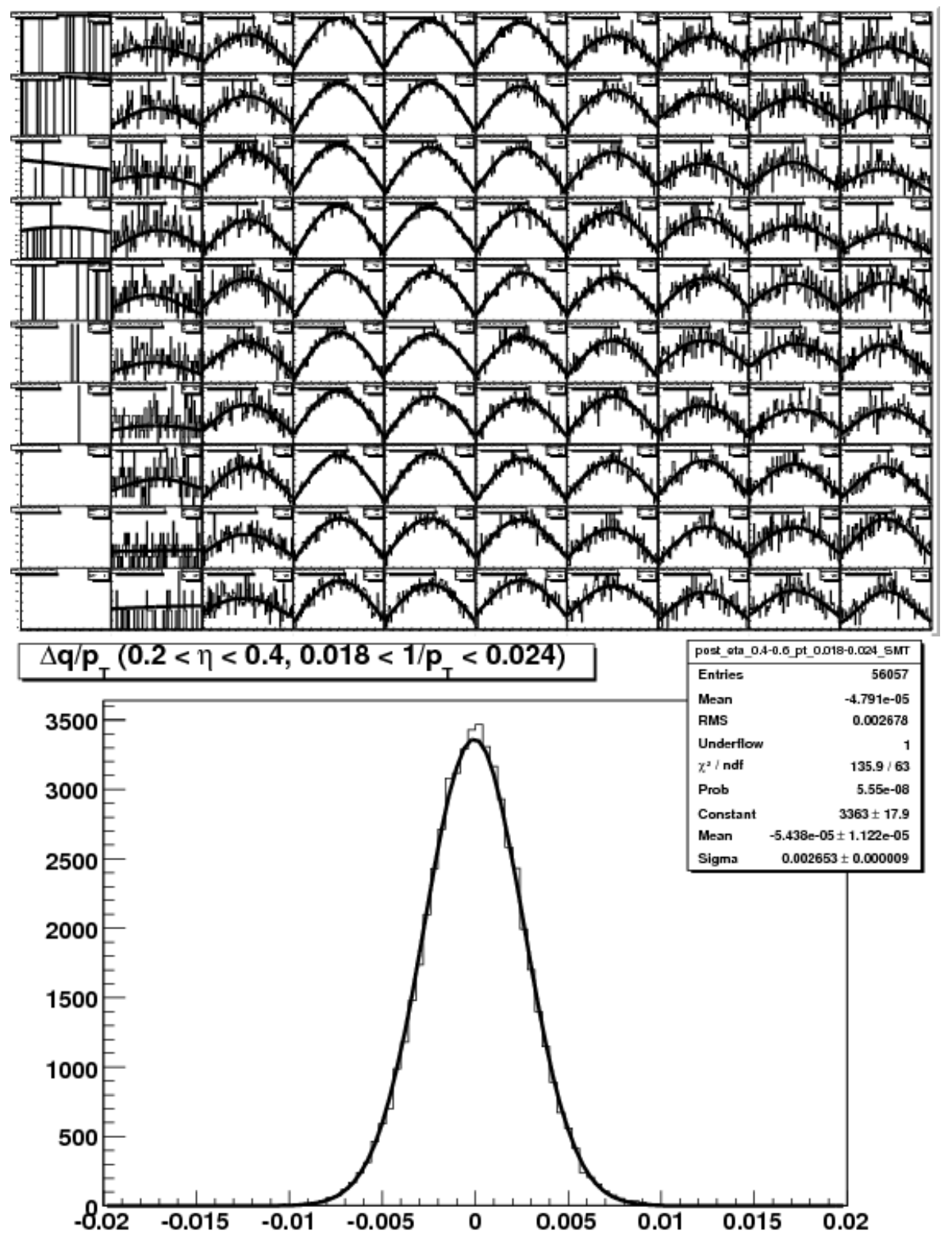

Figure B.1: Above: $\Delta \frac{q}{p_{T}}$ distributions for post-shutdown data with SMT hits. On the left, each histogram bins the MC by $|\eta|$ (with 0.0 at the top and 2.0 at the bottom) and $\frac{1}{p_{T}}$ (with $0.0 \mathrm{GeV}^{-1}$ on the left and $0.6 \mathrm{GeV}^{-1}$ on the right). Below: The distribution for $0.2<\eta<0.4$ and $0.018<\frac{1}{p_{T}}<0.024 \mathrm{GeV}^{-1}$.

without SMT hits. The results are listed in Table B.1. 


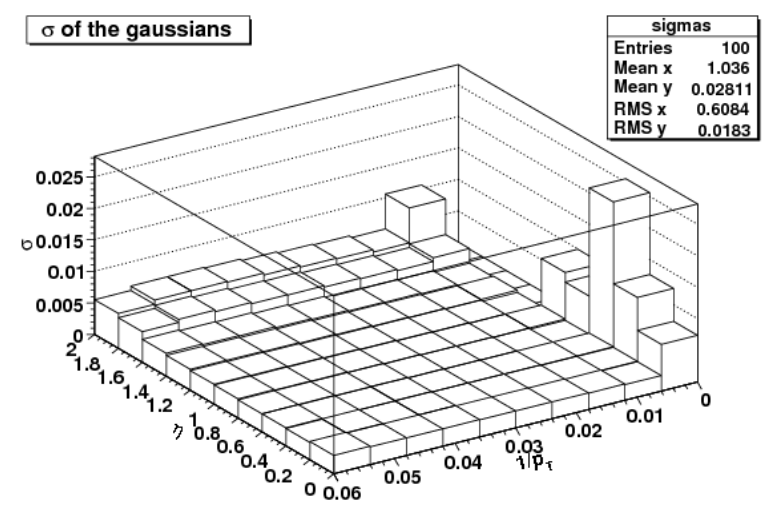

Figure B.2: Width of the Gaussian fits for each of the $10 \eta$ and $101 / p_{T}$ bins of the post-shutdown data with SMT hits.

\section{B.2 Electron $p_{T}$ Uncertainty}

The electron functions were also are extracted from the $\mathbb{E}_{T}$ significance processor ( caf_util

p21-br-98

), used in other DØ analyses. The parametrizations are a function of energy and $\eta$ for the CC and EC north and EC south calorimeters.

The form of the function is:

$$
\frac{\sigma(E)}{E}=\sqrt{C^{2}+S^{2} / E+N^{2} / E^{2}} * E
$$

Where $C$, the constant term, and $N$, the noise term, are coefficients listed in Table B.2. $S$, the sampling term, is given by polynomial function derived using GEANT MC.

\section{B.3 Jet $p_{T}$ Uncertainty}

The jet resolution functions were also are extracted from the $\mathbb{E}_{T}$ significance processor ( caf_util

p21-br-98

). The parametrizations are a function of $p_{T}$ in bins of rapidity, with coefficients listed in Table B.3. The parametrization used is:

$$
\frac{\sigma\left(p_{T}\right)}{p_{T}}=\sqrt{C^{2}+S^{2} / p_{T}+N^{2} p_{T}^{2}}
$$




\begin{tabular}{||c|c|c||}
\hline \hline & RunIla pre with smt & RunIla pre without smt \\
\hline$c_{0}$ & 0.00244128 & 0.00247744 \\
$c_{1}$ & 0.0101999 & 0.00435522 \\
$\sigma_{0}$ & 0.00677823 & 0.00915502 \\
$\sigma_{1}$ & 0.0483866 & 0.023103 \\
\hline \hline & RunIIa post with smt & RunIIa post without smt \\
\hline$c_{0}$ & 0.00244113 & 0.00247739 \\
$c_{1}$ & 0.010204 & 0.00435678 \\
$\sigma_{0}$ & 0.00677562 & 0.00914945 \\
$\sigma_{1}$ & 0.0485938 & 0.0234536 \\
\hline \hline & RunIIb with smt & RunIIb without smt \\
\hline$c_{0}$ & 0.00244128 & 0.00247744 \\
$c_{1}$ & 0.0101999 & 0.00435522 \\
$\sigma_{0}$ & 0.00677823 & 0.00915502 \\
$\sigma_{1}$ & 0.0483866 & 0.023103 \\
\hline \hline
\end{tabular}

Table B.1: transfer functions parameters for the three different epochs

Table B.2: EM energy resolution function coefficients. Since the sampling term is a function, the average is given in this table.

\begin{tabular}{|c|ccc|}
\hline \hline & $C$ & $N$ & Average $S$ \\
\hline$-3.2<\eta<-1.5$ & 0.027 & 0.4 & 0.14 \\
$-1.1<\eta<1.1$ & 0.02 & 0.4 & 0.1 \\
$1.5<\eta<3.2$ & 0.032 & 0.4 & 0.1 \\
\hline \hline
\end{tabular}

\section{B.4 Lepton and Jet Angular Uncertainties}

The resolutions of the angles, listed in Table B.4, are measured in $Z H$ MC. The angular resolution is assumed not to depend on the $\eta$, $\phi$, or $p_{T}$ of the object. This is a crude approximation, but is sufficient for our purpouses. 
Table B.3: Jet $p_{T}$ resolution function coefficients

\begin{tabular}{|c|ccc|}
\hline \hline & $\mathrm{N}$ & $\mathrm{S}$ & $\mathrm{C}$ \\
\hline$|y|<0.4$ & 2.0673 & 0.7029 & 0.0577 \\
$0.4<|y|<0.8$ & 2.0673 & 0.7829 & 0.0615 \\
$0.8<|y|<1.2$ & 2.0673 & 0.8884 & 0.0915 \\
$1.2<|y|<1.6$ & 2.0673 & 0.6263 & 0.1053 \\
$1.6<|y|<2.0$ & 2.0673 & 0.5850 & 0.0706 \\
$2.0<|y|<2.4$ & 2.0673 & 0.4691 & 0.0713 \\
$2.4<|y|<2.8$ & 2.0673 & 0.4873 & 0.0746 \\
$2.8<|y|<3.2$ & 2.0673 & 0.4005 & 0.0773 \\
$3.2<|y|<3.6$ & 2.0673 & 0.3740 & 0.0801 \\
\hline \hline
\end{tabular}

Table B.4: Muon and Jet angular resolutions

\begin{tabular}{|l|cc|}
\hline \hline & $\eta$ & $\phi$ \\
\hline Muon & 0.002 & 0.001 \\
Jet & 0.08 & 0.08 \\
\hline \hline
\end{tabular}




\section{Appendix $\mathrm{C}$}

\section{Optimization of the $\mathbb{F}_{T}^{\prime} k$ and $k^{\prime}$ multipliers}

The selection variable, $\mathbb{E}_{T}^{\prime}$, is formulated to maximally reject inclusive $Z$ background by optimizing in terms of its lepton $p_{T}$ uncertainty and trackjet corrections. This is done by varying the $k$ and $k^{\prime}$ multipliers in Equation 7.3 and Equation 7.4 such as to increase the value of $\frac{S}{\sqrt{B}}$ in the non-signal region at $25 \mathrm{GeV}$. The effect of these optimizations can be seen in

Figure C.1. In this way we increase the slope of the $\mathbb{E}_{T}^{\prime}$ distribution. The gains due to improved inclusive $Z$ rejection are modest and slightly counteracted by a diminished signal presence.

Table C.1: Multiplier applied to the lepton $p_{T}$ measurement uncertainty when reducing the recoil activity.

\begin{tabular}{|l|cc|cc|}
\hline \hline & \multicolumn{2}{|c|}{ Run IIa } & \multicolumn{2}{c|}{ Run IIb } \\
& di-electron & di-muon & di-electron & di-muon \\
\hline$\delta_{i}^{\ell \ell}$ multiplier & 2.2 & 1.5 & 2.2 & 1.5 \\
\hline \hline
\end{tabular}

Table C.2: Multiplier applied to the track correction when reducing the recoil activity.

\begin{tabular}{|l|cc|cc|}
\hline \hline & \multicolumn{2}{|c|}{ Run IIa } & \multicolumn{2}{c|}{ Run IIb } \\
& di-electrons & di-muon & di-electron & di-muon \\
$\delta_{i}^{\text {trk }}$ multiplier & 2.5 & 1.5 & 2.5 & 1.5 \\
\hline \hline
\end{tabular}



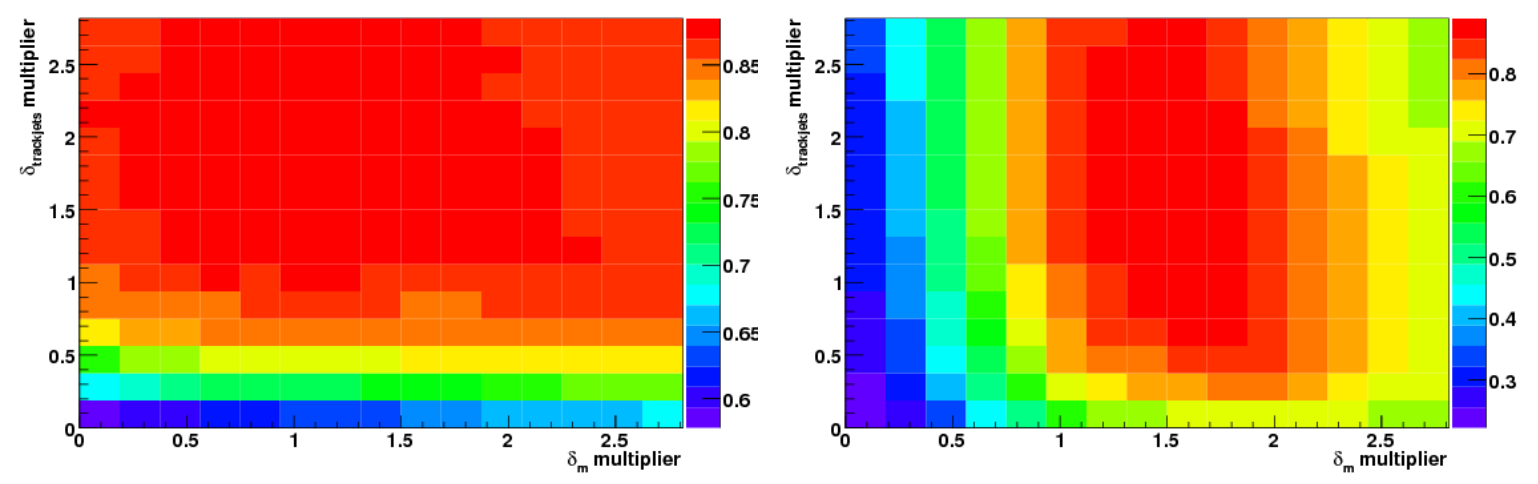

(a) Run IIa dataset
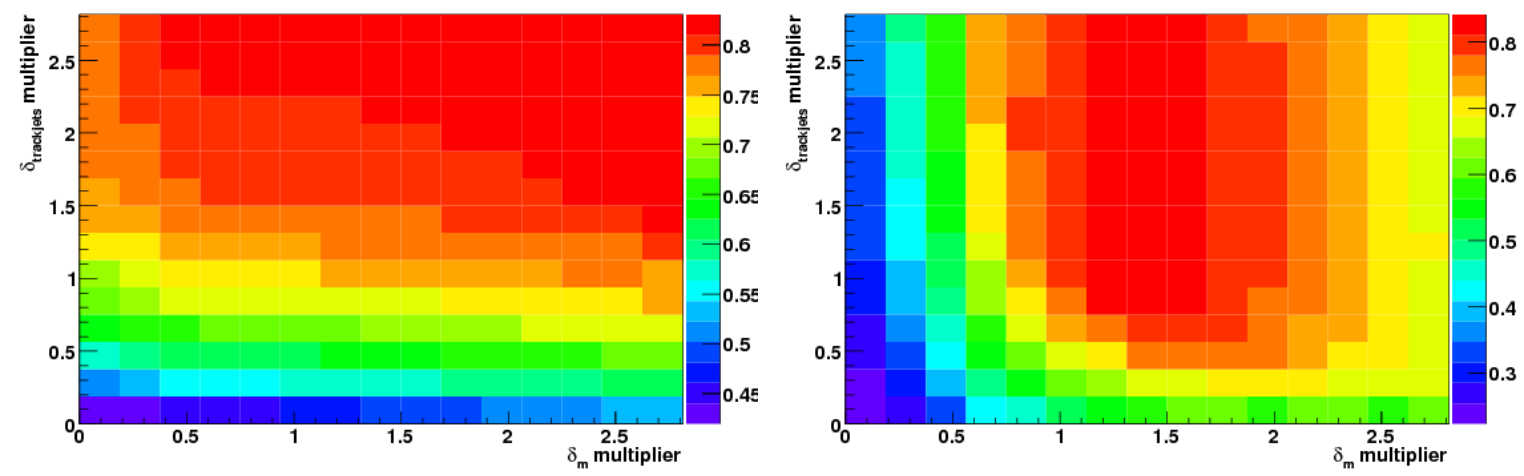

(b) Run IIb dataset

Figure C.1: $\frac{S}{\sqrt{B}}$ for the di-electron (left) and di-muon (right) channels in Run IIa (top) and Run IIb (bottom) at $25 \mathrm{GeV}$ varying the $k$ and $k^{\prime}$ multipliers in steps of 0.2. 


\section{Appendix D}

\section{Inclusive Trigger Modeling}

Although no explicit trigger requirement is made in the $Z H$ analysis, we must still correct for the trigger acceptance effects which are not modeled by the MC. Although there are pre-existing trigger corrections for a number of trigger suites which would do just this, an explicit trigger requirement results in a $\approx 20 \%$ decrease in yield under the $Z$ peak after the two jet cut, as seen in Table D.1.

Table D.1: Trigger yields in RunIIb Data for multiple trigger suites

\begin{tabular}{|l|cc|cc|}
\hline \hline & \multicolumn{2}{|c|}{ Inclusive Jet } & \multicolumn{2}{c|}{$\geq 2$ Jets } \\
\hline Trigger Suite & Inclusive $M_{\ell \ell}$ & $70<M_{\ell \ell}<130$ & Inclusive $M_{\ell \ell}$ & $70<M_{\ell \ell}<130$ \\
\hline Inclusive & 11607 & 10495 & 1156 & 974 \\
single-muon & 1053 & 9601 & 856 & 740 \\
di-muon and single-muon & 10721 & 9765 & 890 & 767 \\
muon + jets and single-muon & 10592 & 9650 & 902 & 775 \\
\hline \hline
\end{tabular}

This issue is resolved using an over-correction derived by examining the data in which no explicit trigger requirement was made ("inclusive") and the data in which a suite of single muon and muon+jet trigger have been used ("exclusive"). Any differences observed in the physics distributions of the two datasets are assumed to be caused entirely by trigger effects. The ratios of these distributions, shown in Figures D.1, D.2, and D.3, are used to derive an ad-hoc correction applied to the $\mathrm{MC}$ on top of the standard trigger efficiencies from Section 3.3.

The single-muon triggers in the $\left|\eta_{\text {det }}\right|>1.6$ region are heavily prescaled, and thus the trigger matching terms reject all data in the 0 jet bin. Because of this, the $\eta_{\text {det }}$ correction is measured after the 2 jet cut. All other corrections are measured in the higher statistics inclusive jet sample.

An $\eta_{\text {det }}$ correction is applied in the forward region, whereas a $p_{T}$ correction is applied in the central region with an additoinal $\phi$ correction in the bottom hole as follows:

- $\left|\eta_{\text {det }}\right|<1.6: \frac{1}{s f}=A_{1}+B_{1} * \exp \left(C_{1} * p_{T}\right)$ 

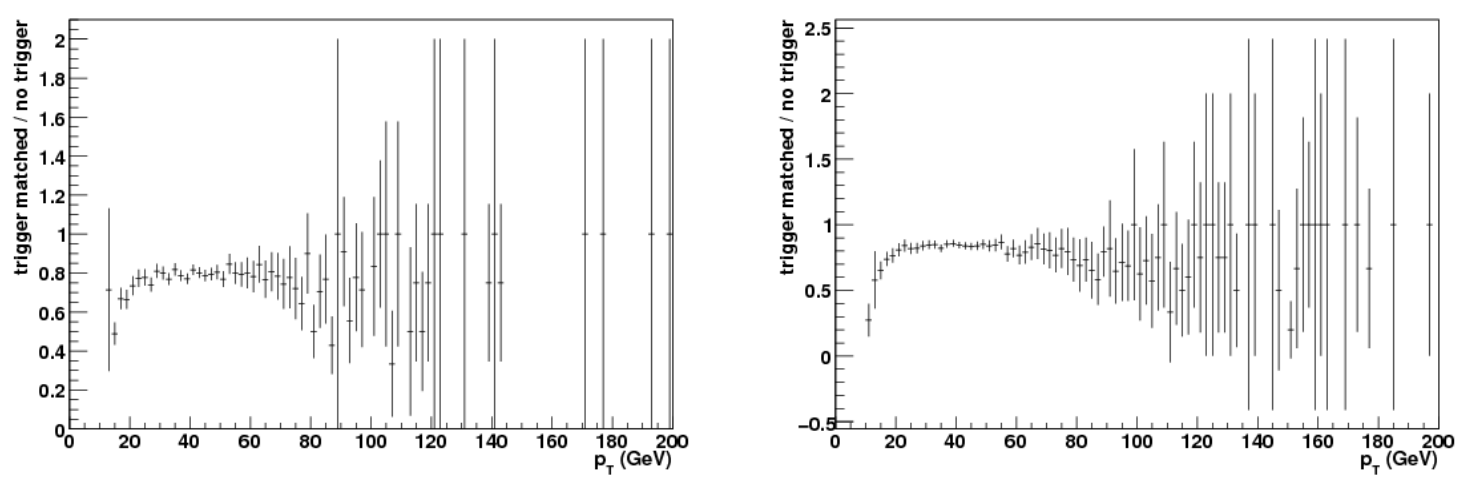

Figure D.1: Ratio of the events in the exclusively and inclusively triggered data samples against muon $p_{T}$ in Run IIa (left) and Run IIb (right).
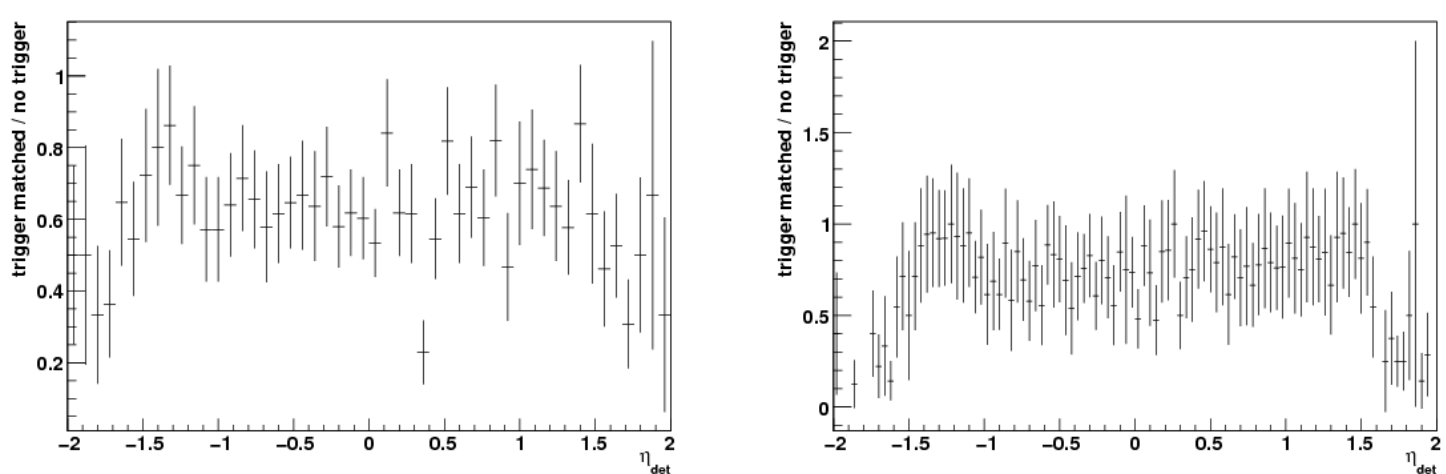

Figure D.2: Ratio of the events in the exclusively and inclusively triggered data samples against muon $\eta_{\text {det }}$ in Run IIa (left) and Run IIb (right).

- $\eta_{\mathrm{det}}<-1.6: \frac{1}{s f}=A_{2}+B_{2} * \eta_{\mathrm{det}}$

- $\eta_{\text {det }}>1.6: \frac{1}{s f}=A_{3}+B_{3} * \eta_{\text {det }}$

- $4.3<\phi<5.2$ and $\left|\eta_{\text {det }}\right|<-1.6: \frac{1}{s f} *=A_{4}$

Applying these corrections results in an oversmearing of the $Z p_{T}$ distribution. To isolate this effect, we apply the ad-hoc correction to the exclusive data and re-compute the ratio to the inclusive data. The ratios are shown in Figure D.4. In the region $Z p_{T}>80(\mathrm{GeV})$ the distribution is consistent with a straight line with no slope. Below that point we derive a final correction which accounts for the correlation between the $\eta, \phi$, and $p_{T}$ of the muon and track. In Run IIa we fit the ratio using a fourth order polynomial, in Run IIb we use a sixth order polynomial. 

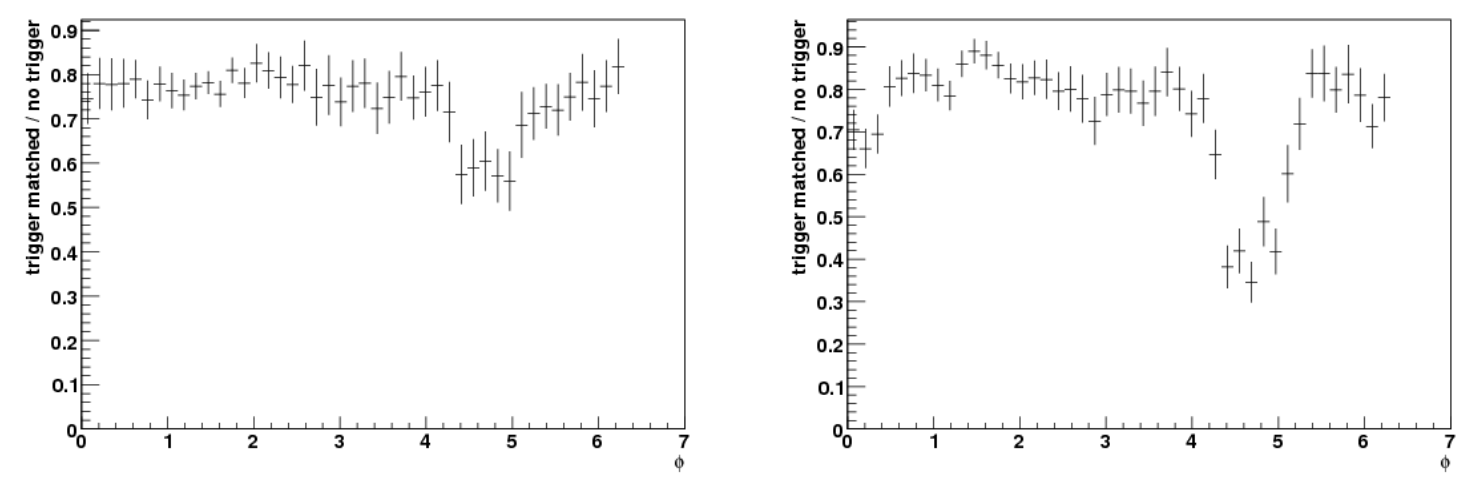

Figure D.3: Ratio of the events in the exclusively and inclusively triggered data samples against muon $\phi$ in Run IIa (left) and Run IIb (right).
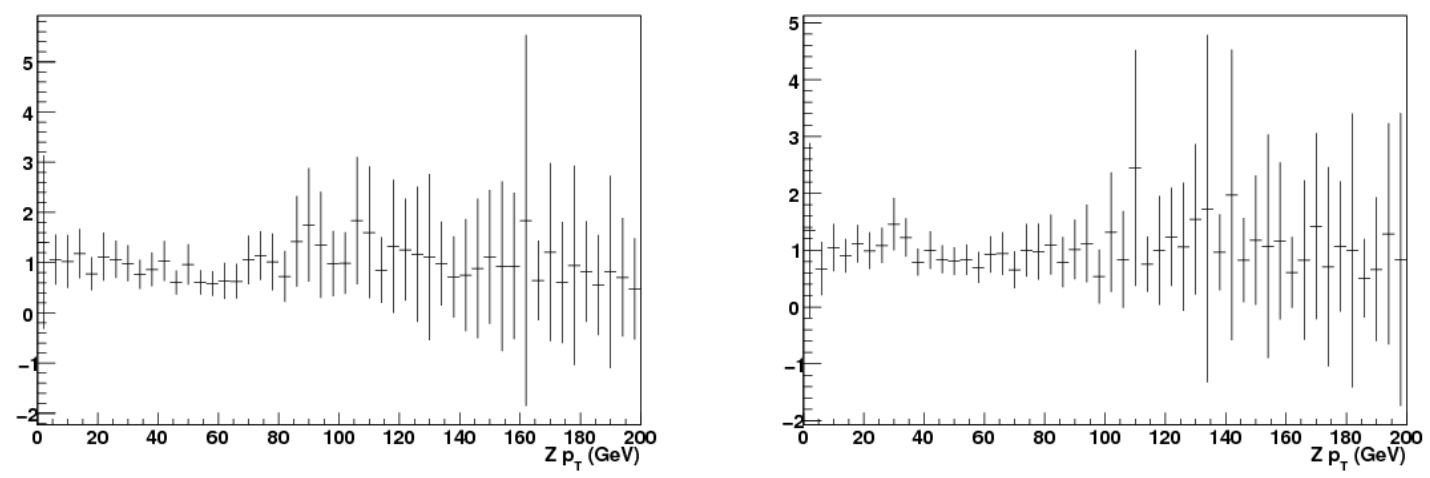

Figure D.4: Ratio of the events in the exclusively and $\eta, \phi, p_{T}$ corrected inclusively triggered muon+track data samples against $Z p_{T}$ in Run IIa (left) and Run IIb (right). 
Table D.2: Trigger Correction Coefficients

\begin{tabular}{|c|ccc|}
\multicolumn{4}{c}{ Run IIa } \\
\hline \hline$i$ & $A_{i}$ & $B_{i}$ & $C_{i}$ \\
\hline 1 & 0.95 & -15.0 & -0.26 \\
2 & 039 & -0.10 & \\
3 & 0.48 & 0.046 & \\
4 & 0.64 & & \\
\hline \hline
\end{tabular}

\begin{tabular}{c|ccc|}
\hline \multicolumn{4}{c}{ Run IIb } \\
\hline \hline$i$ & $A_{i}$ & $B_{i}$ & $C_{i}$ \\
\hline 1 & 0.95 & -8.8 & -0.24 \\
2 & -0.74 & -0.62 & \\
3 & -0.61 & 0.56 & \\
4 & 0.47 & & \\
\hline \hline
\end{tabular}

Table D.3: $Z p_{T}$ Correlation Correction Coefficients

\begin{tabular}{|c|c|c|}
\hline \hline & Run IIa & Run IIb \\
\hline 0 & $8.24 * 10^{-1}$ & 1.14 \\
1 & $2.30 * 10^{-2}$ & $-9.58 * 10^{-2}$ \\
2 & $-6.07 * 10^{-4}$ & $1.11 * 10^{-2}$ \\
3 & $5.23 * 10^{-6}$ & $-4.62 * 10^{-4}$ \\
4 & $-1.44 * 10^{-8}$ & $8.72 * 10^{-6}$ \\
5 & & $-7.66 * 10^{-8}$ \\
6 & & $2.55 * 10^{-10}$ \\
\hline \hline
\end{tabular}

